\title{
Cobalt-Catalyzed Regio- and Enantioselective Allylic
}

\section{Amination}

Samir Ghorai, ${ }^{\dagger}$ Sahadev Shrihari Chirke, ${ }^{\dagger}$ Wen-Bin Xu, ${ }^{\dagger}$ Jia-Feng Chen,${ }^{\dagger}$ Changkun Li* ${ }^{\dagger \dagger}$

${ }^{\dagger}$ Shanghai Key Laboratory for Molecular Engineering of Chiral Drugs, School of Chemistry and Chemical Engineering Shanghai Jiao Tong University, China

Beijing National Laboratory of Molecular Sciences (BNLMS), Beijing 100871, China

$\begin{array}{ll}\text { 1. General information } & \text { S2 }\end{array}$

2. Procedure for the ligand synthesis $\quad$ S3

$\begin{array}{lr}\text { 3. Substrate compounds } & \text { S9 }\end{array}$

4. Optimization of ligands $\quad$ S11

5. Optimization for Cobalt salts and solvents with L6 $\quad$ S12

6. Representative procedure of CoAAA Reaction with anilines and spectral data S13

$\begin{array}{ll}\text { 7. Optimization for linear allylic carbonate }(E \text { and } Z \text { isomers }) & S 23\end{array}$

8. Representative procedure of CoAAA Reaction of aliphatic amines and spectral data S24

$\begin{array}{ll}\text { 9. Gram scale synthesis of 3ab } & \text { S29 }\end{array}$

10. Procedure for the complex preparation and single crystal X-ray diffraction data $\quad$ S30

11. Procedure of Co-Catalyzed AAA Reaction with $\left[\mathrm{LBCo}\left(\mathrm{CH}_{3} \mathrm{CN}\right)_{2}\right]\left(\mathrm{BF}_{4}\right)_{2}$ and $\mathrm{L4}$-CoI $\quad$ S36

12. NMR Spectra of new compounds $\quad S 38$

13. HPLC spectra of chiral compounds $\quad S 85$

$\begin{array}{ll}\text { 14. References } & \text { S113 }\end{array}$ 


\section{General information:}

All air and moisture sensitive manipulations were carried out with standard Schlenk technique or in a nitrogenfilled glove box. Dried and oxygen free solvents were obtained from solvent purification system (Vigor YJC-7) and used thereafter. Column chromatography was performed using 200-300 mesh silica gels. The NMR spectra were recorded on a Bruker-400/500 MHz instrument and chemical shifts are reported in ppm relative to the residual deuterated solvents. High-resolution mass spectra (HRMS) were performed at Instrumental Analys is Center of Shanghai Jiao Tong University with electrospray spectrometer Waters Micromass Q-TOF Premier Mass Spectrometer. Enantiomers ratio were determined by Shimazu HPLC-16 and 2030 instrument using DAICEL chiral column at $40{ }^{\circ} \mathrm{C}$. Melting points were measured with Hanon MP100 melting point apparatus. Optical rotations were measured on an Anton Paar MCP100 automatic polarimeter using a $100 \mathrm{~mm}$ path-length cell at $589 \mathrm{~nm}$. Anhydrous grade $\mathrm{Co}\left(\mathrm{BF}_{4}\right)_{2}(98+\%)$ was purchased from Alfa Aesar. All other chemicals were purchased from TCI, Energy Chemical, Macklin or Alfa Aesar. Solvents were obtained from tansoole, China. Liquid substrates were distilled and degassed prior to use. 


\section{Procedure for the ligand synthesis:}

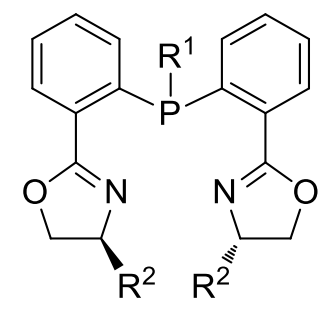

$\mathbf{L 2}\left[\mathrm{R}^{1}=\mathrm{Ph}, \mathrm{R}^{2}={ }^{\mathrm{i}} \mathrm{Pr}\right] ; \mathbf{L} 3\left[\mathrm{R}^{1}=\mathrm{Ph}, \mathrm{R}^{2}={ }^{\mathrm{t}} \mathrm{Bu}\right] ; \mathbf{L} 4\left[\mathrm{R}^{1}=\mathrm{Ph}, \mathrm{R}^{2}=\mathrm{Me}\right] ; \mathbf{L} 5\left[\mathrm{R}^{1}=\mathrm{Ph}, \mathrm{R}^{2}=\mathrm{Bn}\right] ; \mathbf{L 6}\left[\mathrm{R}^{1}=\mathrm{Ph}, \mathrm{R}^{2}\right.$ $=\mathrm{Ph}]$ were synthesizd according to the procedure General Method 1 .

$\mathbf{L 7}\left[\mathrm{R}^{1}=4-\mathrm{CF}_{3} \mathrm{C}_{6} \mathrm{H}_{4}, \mathrm{R}^{2}={ }^{\mathrm{i}} \mathrm{Pr}\right] ; \mathbf{L 8}\left[\mathrm{R}^{1}=4-\mathrm{NMe}_{2} \mathrm{C}_{6} \mathrm{H}_{4}, \mathrm{R}^{2}={ }^{\mathrm{i}} \mathrm{Pr}\right] ; \mathbf{L 9}\left[\mathrm{R}^{1}=4-\mathrm{OMeC}_{6} \mathrm{H}_{4}, \mathrm{R}^{2}={ }^{\mathrm{i}} \operatorname{Pr}\right] ; \mathbf{L 1 0}\left[\mathrm{R}^{1}=\right.$ 4-OMe-3,5- $\left.{ }^{\mathrm{t}} \mathrm{Bu}_{2} \mathrm{C}_{6} \mathrm{H}_{2}, \mathrm{R}^{2}={ }^{\mathrm{i}} \mathrm{Pr}\right] ; \mathbf{L} 11\left[\mathrm{R}^{1}=4-\mathrm{OMe}-3,5-{ }^{\mathrm{t}} \mathrm{Bu}_{2} \mathrm{C}_{6} \mathrm{H}_{2}, \mathrm{R}^{2}=\mathrm{Bn}\right]$ were synthesizd according to the procedure General Method 2.

General Method 1: In a flame dried shlenk tube, 2-bromooxazoline substrate $(8.8 \mathrm{mmol}, 2.2 \mathrm{eq})$ was dissolved in THF $(18 \mathrm{~mL})$ under an argon atmosphere and cooled down to $-78{ }^{\circ} \mathrm{C} .{ }^{\mathrm{n}} \mathrm{BuLi}(2.4 \mathrm{eq}, 1.6 \mathrm{M}$ in hexane) was added dropwise and the mixture was stirred for $1 \mathrm{~h}$ at this temperature. Then phenylphosphine dichloride (4.0 mmol, $1.0 \mathrm{eq}$ ) in $3 \mathrm{~mL}$ of THF was added slowly. The reaction mixture was stirred at $-78^{\circ} \mathrm{C}$ for one hour and warmed to room temperature. After 12 hours, the reaction mixture was quenched with water and extracted with ethyl acetate. Organic phase was washed with brine and dried over $\mathrm{Na}_{2} \mathrm{SO}_{4}$. Solvent was removed under reduced pressure and crude product was purified by flash column chromatography on silica gel using petrolium ether/ethyl acetate as eluent to obtain the pure product.

General Method 2: In a flame dried shlenk tube, 2-bromooxazoline substrate $(8.0 \mathrm{mmol}, 2.0$ eq $)$ was dissolved in THF solvent (16 mL) under an argon atmosphere and cooled down to $-78{ }^{\circ} \mathrm{C}$. ${ }^{\mathrm{n}} \mathrm{BuLi}(2.4 \mathrm{eq}, 1.6 \mathrm{M}$ in hexane) was added dropwise and the mixture was stirred for $1 \mathrm{~h}$ at this temperature. The reaction mixture was further cooled to $\sim-100{ }^{\circ} \mathrm{C}$ and was added triphenylphosphite $(4.0 \mathrm{mmol}, 1.0 \mathrm{eq})$ in $2.0 \mathrm{~mL}$ of THF in one portion under vigorous stirring. The reaction was slowly warmed up to room temperature and stirred for 5 hours. Phosphinite reaction mixture was used in situ for further substitution to get the desired ligand.

In another flame dried shlenk tube, lithiation of the substituted benzene was carried out from their corresponding bromo-derivative $(4.8 \mathrm{mmol}, 1.2 \mathrm{eq})$ using ${ }^{\mathrm{n}} \mathrm{BuLi}(1.3 \mathrm{eq})$ at $-78{ }^{\circ} \mathrm{C}$. Then the reaction mixture was added to the phosphinite solution via syringe over 10 minutes. Combined reaction mixture was allowed to warm to room temperature slowly and stirred overnight. The reaction mixture was quenched with water and extracted with ethyl acetate. The solvent was removed and the residue was purified by flash column chromatography on silica gel using petrolium ether/ethyl acetate as eluent to obtain the pure product.

L12 was synthesized according to the literature reported procedure. ${ }^{1}$ 
$\mathbf{L} 2\left[\mathbf{R}^{1}=\mathbf{P h}, \mathbf{R}^{2}={ }^{\mathrm{i}} \mathbf{P r}\right]:$

White solid; Yield $1.491 \mathrm{~g}, 77 \%$.

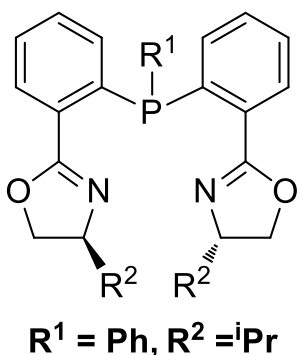

${ }^{1} \mathbf{H}$ NMR $\left(400 \mathrm{MHz}, \mathrm{CDCl}_{3}\right) \delta 7.86(\mathrm{td}, J=7.8,4.4 \mathrm{~Hz}, 2 \mathrm{H}), 7.33-7.23(\mathrm{~m}, 9 \mathrm{H})$, $6.93(\mathrm{ddd}, J=10.5,7.7,3.1 \mathrm{~Hz}, 2 \mathrm{H}), 4.19-4.10(\mathrm{~m}, 2 \mathrm{H}), 3.93-3.86(\mathrm{~m}, 4 \mathrm{H}), 1.56$ (ddq, $J=19.8,13.2,6.6 \mathrm{~Hz}, 2 \mathrm{H}), 0.78(\mathrm{dd}, J=14.1,6.7 \mathrm{~Hz}, 6 \mathrm{H}), 0.71(\mathrm{t}, J=6.3$ $\mathrm{Hz}, 6 \mathrm{H})$.

${ }^{13}$ C NMR $\left(101 \mathrm{MHz}, \mathrm{CDCl}_{3}\right) \delta 163.2(\mathrm{dd}, J=35.4,2.0 \mathrm{~Hz}), 140.3(\mathrm{dd}, J=44.3$, $25.4 \mathrm{~Hz}), 139.1(\mathrm{~d}, J=13.6 \mathrm{~Hz}), 134.7,134.5(\mathrm{~d}, J=2.6 \mathrm{~Hz}), 134.1,132.3(\mathrm{dd}, J=$ 22.3, 3.0 Hz), $130.3(\mathrm{~d}, J=12.9 \mathrm{~Hz}), 129.8(\mathrm{t}, J=3.5 \mathrm{~Hz}), 128.5-128.2(\mathrm{~m}), 127.8(\mathrm{~d}, J=4.0 \mathrm{~Hz}), 73.1(\mathrm{~d}, J$ $=15.7 \mathrm{~Hz}), 70.1(\mathrm{~d}, J=1.7 \mathrm{~Hz}), 32.90,18.9(\mathrm{~d}, J=17.7 \mathrm{~Hz}), 18.5(\mathrm{~d}, J=6.3 \mathrm{~Hz})$.

$[\alpha]_{D}^{25}=-52.1\left(c 1.0, \mathrm{CHCl}_{3}\right)$.

HRMS (ESI): calculated $m / z$ for $\mathrm{C}_{30} \mathrm{H}_{33} \mathrm{~N}_{2} \mathrm{O}_{2} \mathrm{P}[\mathrm{M}+\mathrm{H}]^{+}=485.2352$, found 485.2360.

The analytical data are accordance with the reported literature. ${ }^{2}$

\section{$\mathbf{L 3}\left[\mathbf{R}^{1}=\mathbf{P h}, \mathbf{R}^{2}={ }^{\mathrm{t}} \mathrm{Bu}\right]:$}

White solid; Yield $1.537 \mathrm{~g}, 75 \%$.

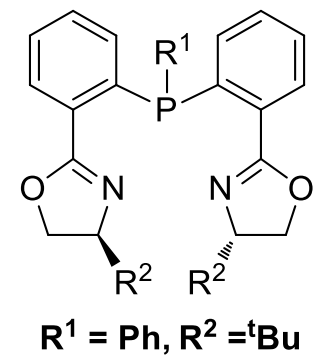

${ }^{1} \mathbf{H}$ NMR $\left(400 \mathrm{MHz}, \mathrm{CDCl}_{3}\right) \delta 7.93-7.87(\mathrm{~m}, 2 \mathrm{H}), 7.35-7.21(\mathrm{~m}, 9 \mathrm{H}), 6.92-$ $6.88(\mathrm{~m}, 2 \mathrm{H}), 4.11-3.97(\mathrm{~m}, 4 \mathrm{H}), 3.87(\mathrm{dt}, J=10.1,8.2 \mathrm{~Hz}, 2 \mathrm{H}), 0.71(\mathrm{~s}, 9 \mathrm{H}), 0.68$ (s, 9H).

${ }^{13}$ C NMR $\left(101 \mathrm{MHz}, \mathrm{CDCl}_{3}\right) \delta 163.0(\mathrm{dd}, J=62.0,2.5 \mathrm{~Hz}), 140.5(\mathrm{dd}, J=75.5$, $26.0 \mathrm{~Hz}), 139.5$ (d, $J=13.5 \mathrm{~Hz}), 134.7$ (d, $J=31.9 \mathrm{~Hz}), 134.3$ (d, $J=9.8 \mathrm{~Hz}), 132.3$ $(\mathrm{dd}, J=22.0,6.4 \mathrm{~Hz}), 130.4(\mathrm{~d}, J=13.5 \mathrm{~Hz}), 129.8(\mathrm{dd}, J=8.1,3.4 \mathrm{~Hz}), 128.4$, $128.3(\mathrm{~d}, J=6.6 \mathrm{~Hz}), 127.9(\mathrm{~d}, J=6.1 \mathrm{~Hz}), 76.8(\mathrm{~d}, J=38.7 \mathrm{~Hz}), 68.4(\mathrm{~d}, J=14.6 \mathrm{~Hz}), 33.8(\mathrm{~d}, J=9.1 \mathrm{~Hz})$, $25.9(\mathrm{~d}, J=6.2 \mathrm{~Hz})$.

$[\alpha]_{D}^{25}=-52.8\left(c 1.0, \mathrm{CHCl}_{3}\right)$.

HRMS (ESI): calculated $m / z$ for $\mathrm{C}_{32} \mathrm{H}_{37} \mathrm{~N}_{2} \mathrm{O}_{2} \mathrm{P}[\mathrm{M}+\mathrm{H}]^{+}=513.2665$, found 513.2683.

The analytical data are accordance with the reported literature. ${ }^{2}$ 


\section{$\mathbf{L} 4\left[\mathbf{R}^{1}=\mathrm{Ph}, \mathbf{R}^{2}=\mathrm{Me}\right]:$}

White solid; Yield $1.370 \mathrm{~g}, 80 \%$.

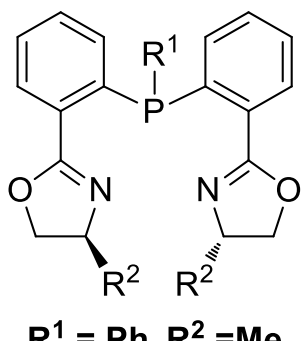

${ }^{1} \mathbf{H}$ NMR $\left(400 \mathrm{MHz}, \mathrm{CDCl}_{3}\right) \delta 7.83-7.80(\mathrm{~m}, 2 \mathrm{H}), 7.38-7.22(\mathrm{~m}, 9 \mathrm{H}), 6.95(\mathrm{ddd}$, $J=7.9,4.2,1.1 \mathrm{~Hz}, 2 \mathrm{H}), 4.30-4.11(\mathrm{~m}, 4 \mathrm{H}), 3.67(\mathrm{dt}, J=15.6,7.6 \mathrm{~Hz}, 2 \mathrm{H}), 1.07$ $(\mathrm{dd}, J=8.7,6.5 \mathrm{~Hz}, 6 \mathrm{H})$.

${ }^{13}$ C NMR $\left(101 \mathrm{MHz}, \mathrm{CDCl}_{3}\right) \delta 163.9(\mathrm{~d}, J=17.0 \mathrm{~Hz}), 139.9-139.7(\mathrm{~m}), 138.3(\mathrm{~d}$, $J=13.8 \mathrm{~Hz}), 134.8(\mathrm{~d}, J=22.2 \mathrm{~Hz}), 134.0(\mathrm{~d}, J=40.1 \mathrm{~Hz}), 132.5(\mathrm{dd}, J=39.2$, $22.8 \mathrm{~Hz}), 130.4(\mathrm{~d}, J=11.3 \mathrm{~Hz}), 129.9(\mathrm{dd}, J=10.5,4.0 \mathrm{~Hz}), 128.7,128.5(\mathrm{~d}, J=$ $7.6 \mathrm{~Hz}), 128.0(\mathrm{~d}, J=11.3 \mathrm{~Hz}), 73.9(\mathrm{~d}, J=2.5 \mathrm{~Hz}), 62.2(\mathrm{~d}, J=8.7 \mathrm{~Hz}), 21.2(\mathrm{~d}, J=6.3 \mathrm{~Hz})$.

$[\alpha]_{D}^{25}=-25.5\left(c 1.0, \mathrm{CHCl}_{3}\right)$.

HRMS (ESI): calculated $m / z$ for $\mathrm{C}_{26} \mathrm{H}_{25} \mathrm{~N}_{2} \mathrm{O}_{2} \mathrm{P}[\mathrm{M}+\mathrm{H}]^{+}=429.1726$, found 429.1736 .

The analytical data are accordance with the reported literature. ${ }^{2}$

\section{$\mathbf{L 5}\left[\mathbf{R}^{1}=\mathbf{P h}, \mathbf{R}^{2}=\mathbf{B n}\right]:$}

White solid; Yield $1.532 \mathrm{~g}, 66 \%$.

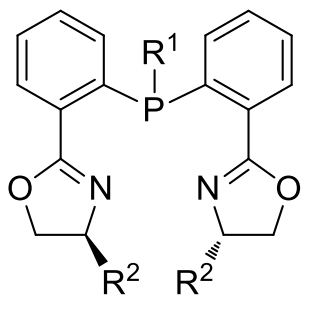

${ }^{1} \mathbf{H}$ NMR (400 MHz, $\left.\mathrm{CDCl}_{3}\right) \delta 7.89-7.82(\mathrm{~m}, 2 \mathrm{H}), 7.36-7.08$ (m, 19H), $7.03-$ $6.97(\mathrm{~m}, 2 \mathrm{H}), 4.46-4.38(\mathrm{~m}, 2 \mathrm{H}), 4.11(\mathrm{dd}, J=19.0,9.1 \mathrm{~Hz}, 2 \mathrm{H}), 3.89(\mathrm{dd}, J=$ 15.5, 7.3 Hz, 2H), 2.99 (ddd, $J=19.2,13.8,5.2 \mathrm{~Hz}, 2 \mathrm{H}), 2.39-2.29(\mathrm{~m}, 2 \mathrm{H})$.

${ }^{13}$ C NMR $\left(101 \mathrm{MHz}, \mathrm{CDCl}_{3}\right) \delta 164.2(\mathrm{dd}, J=11.2,1.8 \mathrm{~Hz}), 140.1(\mathrm{dd}, J=25.0$, $20.7 \mathrm{~Hz}), 138.5$ (t, $J=8.2 \mathrm{~Hz}), 134.9-134.5(\mathrm{~m}), 134.0,132.4$ (dd, $J=29.9,22.3$ $R^{1}=P h, R^{2}=B n$

$\mathrm{Hz}), 130.6(\mathrm{~d}, J=17.4 \mathrm{~Hz}), 130.0(\mathrm{dd}, J=8.1,3.7 \mathrm{~Hz}), 129.4(\mathrm{~d}, J=4.8 \mathrm{~Hz}), 128.7$ $(\mathrm{d}, J=9.7 \mathrm{~Hz}), 128.6(\mathrm{~d}, J=3.1 \mathrm{~Hz}), 128.1(\mathrm{~d}, J=5.2 \mathrm{~Hz}), 126.4(\mathrm{~d}, J=2.8 \mathrm{~Hz}), 71.8(\mathrm{~d}, J=4.8 \mathrm{~Hz}), 68.2$, $41.5(\mathrm{~d}, J=2.4 \mathrm{~Hz})$.

${ }^{31}$ P NMR $\left(162 \mathrm{MHz}, \mathrm{CDCl}_{3}\right) \delta-7.00$.

$[\alpha]_{D}^{25}=+17.3\left(c 1.0, \mathrm{CHCl}_{3}\right)$

HRMS (ESI): calculated $m / z$ for $\mathrm{C}_{38} \mathrm{H}_{33} \mathrm{~N}_{2} \mathrm{O}_{2} \mathrm{P}[\mathrm{M}+\mathrm{H}]^{+}=581.2352$, found 581.2364. 
$\mathbf{L 6}\left[\mathbf{R}^{1}=\mathrm{Ph}, \mathbf{R}^{2}=\mathrm{Ph}\right]:$

White solid; Yield $1.215 \mathrm{~g}, 55 \%$.

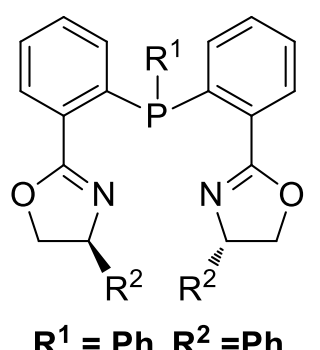

${ }^{1} \mathbf{H}$ NMR $\left(400 \mathrm{MHz}, \mathrm{CDCl}_{3}\right) \delta 8.02-7.97(\mathrm{~m}, 2 \mathrm{H}), 7.41-7.33(\mathrm{~m}, 9 \mathrm{H}), 7.23-$ $7.17(\mathrm{~m}, 6 \mathrm{H}), 7.08-6.98(\mathrm{~m}, 6 \mathrm{H}), 5.28-5.21(\mathrm{~m}, 2 \mathrm{H}), 4.58(\mathrm{q}, J=7.0 \mathrm{~Hz}, 2 \mathrm{H})$, $4.00(\mathrm{td}, J=8.5,4.4 \mathrm{~Hz}, 2 \mathrm{H})$.

${ }^{13}$ C NMR $\left(101 \mathrm{MHz}, \mathrm{CDCl}_{3}\right) \delta 164.8(\mathrm{dd}, J=8.4,2.1 \mathrm{~Hz}), 142.5(\mathrm{~d}, J=2.8 \mathrm{~Hz})$, $140.4(\mathrm{dd}, J=25.5,17.4 \mathrm{~Hz}), 138.6(\mathrm{~d}, J=13.8 \mathrm{~Hz}), 134.7(\mathrm{~d}, J=22.1 \mathrm{~Hz}), 134.1$, $132.0(\mathrm{dd}, J=53.5,22.5 \mathrm{~Hz}), 130.8(\mathrm{~d}, J=2.4 \mathrm{~Hz}), 130.3(\mathrm{dd}, J=11.5,3.8 \mathrm{~Hz})$, $128.7(\mathrm{~d}, J=2.5 \mathrm{~Hz}), 128.7,128.5(\mathrm{~d}, J=6.3 \mathrm{~Hz}), 128.1(\mathrm{~d}, J=5.2 \mathrm{~Hz}), 127.2(\mathrm{~d}, J=4.6 \mathrm{~Hz}), 126.8(\mathrm{~d}, J=$ $11.1 \mathrm{~Hz}), 74.5,70.2(\mathrm{~d}, J=6.7 \mathrm{~Hz})$.

${ }^{31}$ P NMR $\left(162 \mathrm{MHz}, \mathrm{CDCl}_{3}\right) \delta-7.11$. $[\alpha]_{D}^{25}=+37.6\left(c 1.0, \mathrm{CHCl}_{3}\right)$

HRMS (ESI): calculated $m / z$ for $\mathrm{C}_{36} \mathrm{H}_{29} \mathrm{~N}_{2} \mathrm{O}_{2} \mathrm{P}[\mathrm{M}+\mathrm{H}]^{+}=553.2039$, found 553.2058.

\section{$\left.\mathbf{L} 7 \mathbf{R}^{1}=4-\mathrm{CF}_{3} \mathbf{C}_{6} \mathbf{H}_{4}, \mathbf{R}^{2}={ }^{\mathrm{i}} \mathbf{P r}\right]:$}

White solid; Yield $1.590 \mathrm{~g}, 72 \%$.

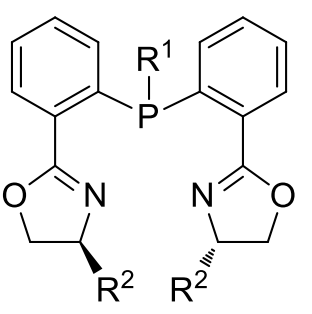

$\mathrm{R}^{1}=4-\mathrm{CF}_{3} \mathrm{C}_{6} \mathrm{H}_{4}$ $\mathbf{R}^{2}={ }^{\mathrm{i}} \mathrm{Pr}$

${ }^{1} \mathbf{H}$ NMR $\left(400 \mathrm{MHz}, \mathrm{CDCl}_{3}\right) \delta 7.90(\mathrm{dd}, J=11.0,7.4 \mathrm{~Hz}, 2 \mathrm{H}), 7.52(\mathrm{~d}, J=7.9 \mathrm{~Hz}$, $2 \mathrm{H}), 7.40-7.24(6 \mathrm{H}), 4.23-4.13(\mathrm{~m}, 2 \mathrm{H}), 3.97-3.85(\mathrm{~m}, 4 \mathrm{H}), 1.51(\mathrm{ddq}, J=26.2$, $13.2,6.6 \mathrm{~Hz}, 2 \mathrm{H}), 0.71(\mathrm{dd}, J=6.7,3.3 \mathrm{~Hz}, 6 \mathrm{H}), 0.67(\mathrm{t}, J=6.9 \mathrm{~Hz}, 6 \mathrm{H})$.

${ }^{13}$ C NMR (101 MHz, $\left.\mathrm{CDCl}_{3}\right) \delta 162.7(\mathrm{dd}, J=20.0,2.5 \mathrm{~Hz}), 145.3(\mathrm{~d}, J=17.9 \mathrm{~Hz})$, $139.6(\mathrm{dd}, J=43.1,25.1 \mathrm{~Hz}), 134.7,134.3(\mathrm{~d}, J=30.3 \mathrm{~Hz}), 132.2(\mathrm{dd}, J=22.3,7.0$ $\mathrm{Hz}), 130.5(\mathrm{~d}, J=12.8 \mathrm{~Hz}), 130.1(\mathrm{~d}, J=32.2 \mathrm{~Hz}), 129.8(\mathrm{t}, J=3.1 \mathrm{~Hz}), 128.3(\mathrm{~d}, J$ $=3.0 \mathrm{~Hz}), 125.7$ (ddd, $J=216.7,189.1,92.5 \mathrm{~Hz}), 125.0(\mathrm{dd}, J=7.1,3.7 \mathrm{~Hz}), 73.3$ (d, $J=17.3 \mathrm{~Hz}), 70.2(\mathrm{~d}, J=11.1 \mathrm{~Hz}), 33.0(\mathrm{~d}, J=4.2 \mathrm{~Hz}), 18.6$ (dd, $J=20.1,14.7 \mathrm{~Hz})$.

${ }^{31}$ P NMR $\left(162 \mathrm{MHz}, \mathrm{CDCl}_{3}\right) \delta-8.06$. $[\alpha]_{D}^{25}=-27.2\left(c 1.0, \mathrm{CHCl}_{3}\right)$.

HRMS (ESI): calculated $m / z$ for $\mathrm{C}_{31} \mathrm{H}_{32} \mathrm{~F}_{3} \mathrm{~N}_{2} \mathrm{O}_{2} \mathrm{P}[\mathrm{M}+\mathrm{H}]^{+}=553.2226$, found 553.2252. 
$\mathbf{L 8}\left[\mathbf{R}^{1}=4-\mathrm{NMe}_{2} \mathrm{C}_{6} \mathbf{H}_{4}, \mathbf{R}^{2}={ }^{\mathrm{i}} \mathrm{Pr}\right]:$

White solid; Yield $1.159 \mathrm{~g}, 55 \%$.

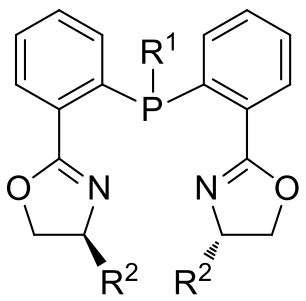

$\mathrm{R}^{1}=4-\mathrm{NMe}_{2} \mathrm{C}_{6} \mathrm{H}_{4}$ $\mathbf{R}^{2}={ }^{\mathrm{i}} \mathrm{Pr}$

${ }^{1} \mathbf{H}$ NMR $\left(400 \mathrm{MHz}, \mathrm{CDCl}_{3}\right) \delta 7.84-7.79(\mathrm{~m}, 2 \mathrm{H}), 7.32-7.19(\mathrm{~m}, 7 \mathrm{H}), 7.01(\mathrm{dd}$, $J=8.9,3.6 \mathrm{~Hz}, 2 \mathrm{H}), 6.67(\mathrm{~d}, J=8.2 \mathrm{~Hz}, 2 \mathrm{H}), 4.17-4.11(\mathrm{~m}, 2 \mathrm{H}), 3.93-3.83(\mathrm{~m}$, $4 \mathrm{H}), 2.94(\mathrm{~s}, 6 \mathrm{H}), 1.66-1.54(\mathrm{~m}, 2 \mathrm{H}), 0.81(\mathrm{~m}, 12 \mathrm{H})$.

${ }^{13}$ C NMR $\left(101 \mathrm{MHz}, \mathrm{CDCl}_{3}\right) \delta 164.0(\mathrm{dd}, J=56.5,1.5 \mathrm{~Hz}), 150.9,141.1(\mathrm{dd}, J=$ 37.7, $24.4 \mathrm{~Hz}), 136.2(\mathrm{~d}, J=23.7 \mathrm{~Hz}), 134.0(\mathrm{~d}, J=33.0 \mathrm{~Hz}), 132.3(\mathrm{dd}, J=22.3$, $13.3 \mathrm{~Hz}), 130.2(\mathrm{~d}, J=7.7 \mathrm{~Hz}), 129.9(\mathrm{dd}, J=7.0,3.7 \mathrm{~Hz}), 127.6,123.0(\mathrm{~d}, J=8.7$ $\mathrm{Hz}), 112.5(\mathrm{~d}, J=8.7 \mathrm{~Hz}), 73.0(\mathrm{~d}, J=9.6 \mathrm{~Hz}), 70.2(\mathrm{~d}, J=17.5 \mathrm{~Hz}), 40.4,32.9$ (d, $J=3.3 \mathrm{~Hz}), 18.8(\mathrm{dd}, J=63.0,19.6 \mathrm{~Hz})$.

${ }^{31} \mathbf{P}$ NMR $\left(162 \mathrm{MHz}, \mathrm{CDCl}_{3}\right) \delta-8.62$.

$[\alpha]_{D}^{25}=-57.2\left(c 1.0, \mathrm{CHCl}_{3}\right)$.

HRMS (ESI): calculated $m / z$ for $\mathrm{C}_{32} \mathrm{H}_{38} \mathrm{~N}_{3} \mathrm{O}_{2} \mathrm{P}[\mathrm{M}+\mathrm{H}]^{+}=528.2774$, Found 528.2803.

L9 $\left[\mathbf{R}^{1}=4-\mathrm{OMeC}_{6} \mathrm{H}_{4}, \mathbf{R}^{2}={ }^{\mathrm{i}} \mathrm{Pr}\right]$ :

White solid; Yield $1.275 \mathrm{~g}, 62 \%$.

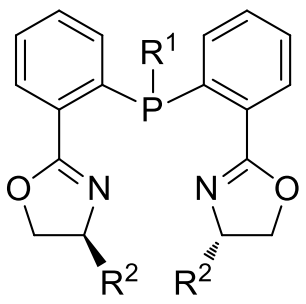

$\mathrm{R}^{1}=4-\mathrm{OMeC}_{6} \mathrm{H}_{4}$ $\mathbf{R}^{2}={ }^{\mathrm{i}} \mathrm{Pr}$

${ }^{1} \mathbf{H}$ NMR $\left(400 \mathrm{MHz}, \mathrm{CDCl}_{3}\right) \delta 7.86-7.81(\mathrm{~m}, 2 \mathrm{H}), 7.32-7.21(\mathrm{~m}, 6 \mathrm{H}), 6.97-$ $6.92(\mathrm{~m}, 2 \mathrm{H}), 6.85(\mathrm{~d}, J=8.3 \mathrm{~Hz}, 2 \mathrm{H}), 4.13(\mathrm{t}, J=12.4 \mathrm{~Hz}, 2 \mathrm{H}), 3.91-3.84(\mathrm{~m}$, $4 \mathrm{H}), 3.78(\mathrm{~s}, 3 \mathrm{H}), 1.57$ (dtt, $J=19.4,13.1,6.6 \mathrm{~Hz}, 2 \mathrm{H}), 0.85-0.70(\mathrm{~m}, 12 \mathrm{H})$.

${ }^{13}$ C NMR $\left(101 \mathrm{MHz}, \mathrm{CDCl}_{3}\right) \delta 163.4(\mathrm{dd}, J=48.0,2.0 \mathrm{~Hz}), 160.1,140.6(\mathrm{dd}, J=$ 38.7, $24.7 \mathrm{~Hz}), 136.3$ (d, $J=23.5 \mathrm{~Hz}), 134.0$ (d, $J=40.6 \mathrm{~Hz}), 132.1$ (d, $J=22.7$ Hz), $130.2(\mathrm{~d}, J=11.4 \mathrm{~Hz}), 129.8(\mathrm{dd}, J=7.5,3.6 \mathrm{~Hz}), 129.4(\mathrm{~d}, J=11.4 \mathrm{~Hz})$, $127.7(\mathrm{~d}, J=3.2 \mathrm{~Hz}), 114.1(\mathrm{~d}, J=8.4 \mathrm{~Hz}), 73.0(\mathrm{~d}, J=16.1 \mathrm{~Hz}), 70.1(\mathrm{~d}, J=9.2$ $\mathrm{Hz}), 55.2,32.9,18.7(\mathrm{dd}, J=48.9,15.8 \mathrm{~Hz})$.

${ }^{31}$ P NMR $\left(162 \mathrm{MHz}, \mathrm{CDCl}_{3}\right) \delta-8.67$.

$[\alpha]_{D}^{25}=-45.5\left(c 1.0, \mathrm{CHCl}_{3}\right)$.

HRMS (ESI): calculated $m / z$ for $\mathrm{C}_{31} \mathrm{H}_{35} \mathrm{~N}_{2} \mathrm{O}_{3} \mathrm{P}[\mathrm{M}+\mathrm{H}]^{+}=515.2464$, found 515.2477.

$\mathbf{L 1 0}\left[\mathbf{R}^{1}=4-\mathrm{OMe}-3,5-{ }^{\mathrm{t}} \mathrm{Bu}_{2} \mathrm{C}_{6} \mathrm{H}_{2}, \mathbf{R}^{2}={ }^{\mathrm{i}} \mathrm{Pr}\right]:$ 
White solid; Yield $1.503 \mathrm{~g}, 60 \%$.

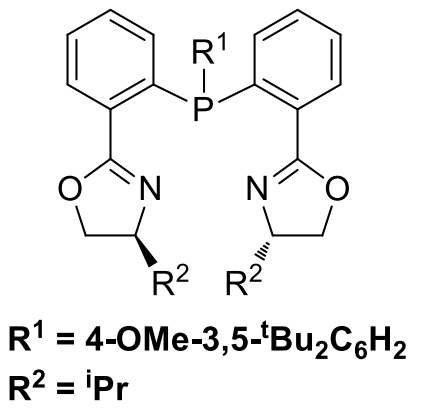

${ }^{1} \mathbf{H}$ NMR $\left(400 \mathrm{MHz}, \mathrm{CDCl}_{3}\right) \delta 7.86(\mathrm{~d}, J=9.6 \mathrm{~Hz}, 2 \mathrm{H}), 7.28(\mathrm{tt}, J=14.8$, $7.4 \mathrm{~Hz}, 4 \mathrm{H}), 7.17(\mathrm{~d}, J=7.8 \mathrm{~Hz}, 2 \mathrm{H}), 6.93(\mathrm{~d}, J=7.1 \mathrm{~Hz}, 2 \mathrm{H}), 4.21-4.11$ $(\mathrm{m}, 2 \mathrm{H}), 3.98-3.85(\mathrm{~m}, 4 \mathrm{H}), 3.67(\mathrm{~s}, 3 \mathrm{H}), 1.61-1.44(\mathrm{~m}, 2 \mathrm{H}), 1.30(\mathrm{~s}$, $18 \mathrm{H}), 0.79-0.72(\mathrm{~m}, 6 \mathrm{H}), 0.69(\mathrm{t}, J=6.7 \mathrm{~Hz}, 6 \mathrm{H})$.

${ }^{13}$ C NMR (101 MHz, $\left.\mathrm{CDCl}_{3}\right) \delta 163.2(\mathrm{dd}, J=9.6,2.4 \mathrm{~Hz}), 160.0(\mathrm{~s}), 143.2$ (d, $J=7.7 \mathrm{~Hz}), 141.2(\mathrm{dd}, J=71.8,25.7 \mathrm{~Hz}), 134.4(\mathrm{~s}), 133.8$ (s), 133.4 (d, $J$ $=23.7 \mathrm{~Hz}), 132.45-131.39(\mathrm{~m}), 130.2(\mathrm{~d}, J=17.3 \mathrm{~Hz}), 129.7(\mathrm{dd}, J=12.2$, $3.3 \mathrm{~Hz}), 127.6(\mathrm{~d}, J=6.4 \mathrm{~Hz}), 73.1(\mathrm{~d}, J=19.9 \mathrm{~Hz}), 70.0(\mathrm{~d}, J=23.5 \mathrm{~Hz}), 64.3(\mathrm{~s}), 35.9(\mathrm{~s}), 33.0(\mathrm{~d}, J=9.1$ $\mathrm{Hz}), 32.2(\mathrm{~s}), 18.7$ (dd, $J=33.5,14.7 \mathrm{~Hz})$.

${ }^{31} \mathbf{P}$ NMR $\left(162 \mathrm{MHz}, \mathrm{CDCl}_{3}\right) \delta-5.76$.

$[\alpha]_{D}^{25}=-42.0\left(c 0.5, \mathrm{CHCl}_{3}\right)$.

HRMS (ESI): calculated $m / z$ for $\mathrm{C}_{39} \mathrm{H}_{51} \mathrm{~N}_{2} \mathrm{O}_{3} \mathrm{P}[\mathrm{M}+\mathrm{H}]^{+}=627.3709$, found 627.3718.

$\mathbf{L 1 1}\left[\mathbf{R}^{1}=4-\mathrm{OMe}-3,5-{ }^{\mathrm{t}} \mathrm{Bu}_{2} \mathrm{C}_{6} \mathrm{H}_{2}, \mathbf{R}^{2}=\mathrm{Bn}\right]:$

White solid; Yield $1.445 \mathrm{~g}, 50 \%$.

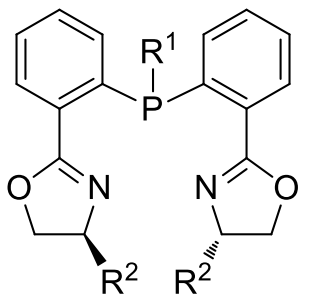

$\mathrm{R}^{1}=4-\mathrm{OMe}-3,5-{ }^{\mathrm{t}} \mathrm{Bu}_{2} \mathrm{C}_{6} \mathrm{H}_{2}$ $\mathrm{R}^{2}=\mathrm{Bn}$

${ }^{1} \mathbf{H}$ NMR $\left(400 \mathrm{MHz}, \mathrm{CDCl}_{3}\right) \delta 7.89-7.82(\mathrm{~m}, 2 \mathrm{H}), 7.34-7.16(\mathrm{~m}, 12 \mathrm{H})$, $7.10(\mathrm{dd}, J=6.7,3.8 \mathrm{~Hz}, 4 \mathrm{H}), 7.00(\mathrm{dd}, J=6.9,3.6 \mathrm{~Hz}, 2 \mathrm{H}), 4.46-4.36(\mathrm{~m}$, 2H), 4.09 (dt, $J=31.6,8.8 \mathrm{~Hz}, 2 \mathrm{H}), 3.92$ (dt, $J=11.1,8.0 \mathrm{~Hz}, 2 \mathrm{H}), 3.66$ (s, $3 \mathrm{H}), 3.01-2.95(\mathrm{~m}, 2 \mathrm{H}), 2.38(\mathrm{dd}, J=13.8,8.8 \mathrm{~Hz}, 1 \mathrm{H}), 2.20(\mathrm{dd}, J=13.7$, $9.3 \mathrm{~Hz}, 1 \mathrm{H}), 1.33$ (s, 18H).

${ }^{13}$ C NMR (101 MHz, $\left.\mathrm{CDCl}_{3}\right) \delta 164.3-164.2(\mathrm{~m}), 160.3,143.5(\mathrm{~d}, J=7.9$ Hz), 140.9 (dd, $J=31.9,25.5 \mathrm{~Hz}), 138.5(\mathrm{~d}, J=9.8 \mathrm{~Hz}), 133.8(\mathrm{t}, J=44.5$ $\mathrm{Hz}), 132.7-131.1(\mathrm{~m}), 130.4(\mathrm{~d}, J=23.1 \mathrm{~Hz}), 129.9(\mathrm{dd}, J=8.8,3.4 \mathrm{~Hz}), 129.3(\mathrm{~d}, J=2.1 \mathrm{~Hz}), 128.6(\mathrm{~d}, J=$ $5.4 \mathrm{~Hz}), 127.8(\mathrm{~d}, J=17.4 \mathrm{~Hz}), 126.4(\mathrm{~d}, J=3.0 \mathrm{~Hz}), 71.6(\mathrm{~d}, J=30.7 \mathrm{~Hz}), 68.2(\mathrm{~d}, J=2.9 \mathrm{~Hz}), 64.4,41.5(\mathrm{~d}$, $J=16.5 \mathrm{~Hz}), 36.0,32.3$.

${ }^{31}$ P NMR $\left(162 \mathrm{MHz}, \mathrm{CDCl}_{3}\right) \delta-5.54$.

$[\alpha]_{D}^{25}=+10.4\left(c 1.0, \mathrm{CHCl}_{3}\right)$ 
HRMS (ESI): calculated $m / z$ for $\mathrm{C}_{47} \mathrm{H}_{51} \mathrm{~N}_{2} \mathrm{O}_{3} \mathrm{P}[\mathrm{M}+\mathrm{H}]^{+}=723.3710$, found 723.3718 .

\section{Substrate compounds}<smiles>C=CC(OCC)C(OC)OC(C)=[In]</smiles>

[1a]<smiles>C=CC(OC(C)=O)C1CC1</smiles>

[1f]<smiles>COC(C)C=CC(C)OC</smiles>

[1b]<smiles>C=CC(CC(C)C)OC(C)=O</smiles>

[1g]<smiles>C=CC(CCc1ccccc1)OC(C)=O</smiles>

[1c]<smiles>C/C=C\C(OC(C)=O)c1ccccc1</smiles>

[1h]<smiles>C=CC(CCC)OC(C)=O</smiles>

[1d]<smiles>C=CC1COC(=O)O1</smiles>

[1i]<smiles>[CH]C(=O)OC(C=C)OC</smiles>

[1e]<smiles>C=CC(CCc1ccccc1)OC(=O)OC(C(F)(F)F)C(F)(F)F</smiles>

[1k]<smiles>O=C(OC/C=C\P=P)OC(C(F)(F)F)C(F)(F)F</smiles>

[4]<smiles>O=C(OC/C=C\P)OC(C(F)(F)F)C(F)(F)F</smiles>

[5]<smiles>Nc1ccccc1</smiles>

[2a]<smiles>Cc1ccccc1N</smiles>

[2f]<smiles>NNc1ccccc1</smiles>

[2k]<smiles>COc1ccc(N)cc1</smiles>

[2b]<smiles>CC(C)(C)c1ccc(N)cc1</smiles>

[2c]<smiles>C=CC(CCCOS(=O)(=O)c1ccccc1)OC(C)=O</smiles>

[1j]<smiles>CCNc1ccc(N)cc1</smiles>

[2g]<smiles>Nc1ccc2[nH]ccc2c1</smiles>

[2h]<smiles>Nc1ccc(Br)cc1</smiles>

[2d]<smiles>CNc1ccccc1</smiles>

[2i]<smiles>CCOC(=O)N1CCNCC1</smiles>

[6b]<smiles>C1CC2(CCN1)OCCO2</smiles>

[6c]<smiles>CC(=O)c1cccc(N)c1</smiles>

[2e]

[6a]<smiles>NCCc1ccccc1</smiles>

[6f]<smiles>C#CCN</smiles>

[6g]<smiles>C[C@H](N)c1ccccc1</smiles>

[6h]

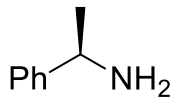

[6i]<smiles>c1ccc2c(c1)CCN2</smiles>

[2j]<smiles>c1ccc2c(c1)CCNC2</smiles>

[6d] [6e]

(6)

$\mathrm{Ph} \widehat{\mathrm{NH}_{2}}$

] 
All the ally lic carbonates (1a to $\mathbf{1 e}$, and $\mathbf{1 g}$ to $\mathbf{1 k}$ ) were prepared according to the reported literature procedure. ${ }^{3}$

\section{1-cyclopropylallyl methyl carbonate (1f):}

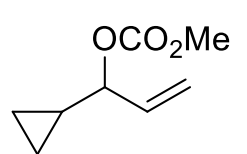

[1f]

Allylic carbonate 1f was synthesized in similar way to the other allylic carbonate reported in the literature. ${ }^{3(a, b, c)}$

${ }^{1} \mathrm{H}$ NMR $\left(500 \mathrm{MHz}, \mathrm{CDCl}_{3}\right) \delta 5.86(\mathrm{ddd}, J=17.0,10.5,6.3 \mathrm{~Hz}, 1 \mathrm{H}), 5.31(\mathrm{~d}, J=17.3 \mathrm{~Hz}$, $1 \mathrm{H}), 5.20(\mathrm{~d}, J=10.6 \mathrm{~Hz}, 1 \mathrm{H}), 4.55-4.49(\mathrm{~m}, 1 \mathrm{H}), 3.77(\mathrm{~s}, 3 \mathrm{H}), 1.17-1.05(\mathrm{~m}, 1 \mathrm{H}), 0.58$ (q, $J=4.6 \mathrm{~Hz}, 2 \mathrm{H}), 0.47(\mathrm{dd}, J=9.3,4.7 \mathrm{~Hz}, 1 \mathrm{H}), 0.35-0.28(\mathrm{~m}, 1 \mathrm{H})$.

${ }^{13} \mathrm{CNMR}\left(126 \mathrm{MHz}, \mathrm{CDCl}_{3}\right) \delta 155.6,135.2,117.4,83.2,54.8,14.7,3.7,2.6$.

\section{(E)-hex-2-en-1-yl (1,1,1,3,3,3-hexafluoropropan-2-yl) carbonate (4):}<smiles>O=C(OC/C=C/P=[In])OC(C(F)(F)F)C(F)(F)F</smiles>

[4]

Allylic carbonate 4 was synthesized following to the literature reported procedure for $1 \mathbf{k}^{3 \mathrm{~d}}$

${ }^{1} \mathrm{H} \mathrm{NMR}\left(400 \mathrm{MHz}, \mathrm{CDCl}_{3}\right) \delta 5.94-5.85(\mathrm{~m}, 1 \mathrm{H}), 5.58(\mathrm{ddd}, J=17.9,12.8,6.4 \mathrm{~Hz}, 2 \mathrm{H})$, $4.70(\mathrm{~d}, J=6.7 \mathrm{~Hz}, 2 \mathrm{H}), 2.06(\mathrm{q}, J=7.1 \mathrm{~Hz}, 2 \mathrm{H}), 1.48-1.36(\mathrm{~m}, 2 \mathrm{H}), 0.90(\mathrm{t}, J=7.4 \mathrm{~Hz}$, $3 \mathrm{H})$.

${ }^{13} \mathrm{C}$ NMR $\left(101 \mathrm{MHz}, \mathrm{CDCl}_{3}\right) \delta 152.9,139.5,123.6(\mathrm{dd}, J=609.4,330.3 \mathrm{~Hz}), 122.1,71.3,70.4(\mathrm{dt}, J=69.9,34.9$ Hz), 34.5, 22.1, 13.7 .

\section{(Z)-hex-2-en-1-yl (1,1,1,3,3,3-hexafluoropropan-2-yl) carbonate (5):}<smiles>CP/C=C\COC(=O)OC(C(F)(F)F)C(F)(F)F</smiles>

[5]

Allylic carbonate 5 was synthesized following to the literature reported procedure for $1 \mathbf{k}^{3 \mathrm{~d}}$

${ }^{1} \mathrm{H}$ NMR $\left(500 \mathrm{MHz}, \mathrm{CDCl}_{3}\right) \delta 5.77(\mathrm{dd}, J=18.5,7.6 \mathrm{~Hz}, 1 \mathrm{H}), 5.63-5.52(\mathrm{~m}$, $2 \mathrm{H}), 4.82(\mathrm{~d}, J=7.1 \mathrm{~Hz}, 2 \mathrm{H}), 2.16-2.07(\mathrm{~m}, 2 \mathrm{H}), 1.42(\mathrm{dd}, J=14.7,7.4 \mathrm{~Hz}, 2 \mathrm{H})$, $0.91(\mathrm{t}, J=7.4 \mathrm{~Hz}, 3 \mathrm{H})$.

${ }^{13} \mathrm{CNMR}\left(126 \mathrm{MHz}, \mathrm{CDCl}_{3}\right) \delta 153.0,137.9,121.5,123.90-116.87(\mathrm{~m}), 72.13-68.65(\mathrm{~m}), 66.2,29.8,22.6,13.8$. 


\section{Optimization of ligands}

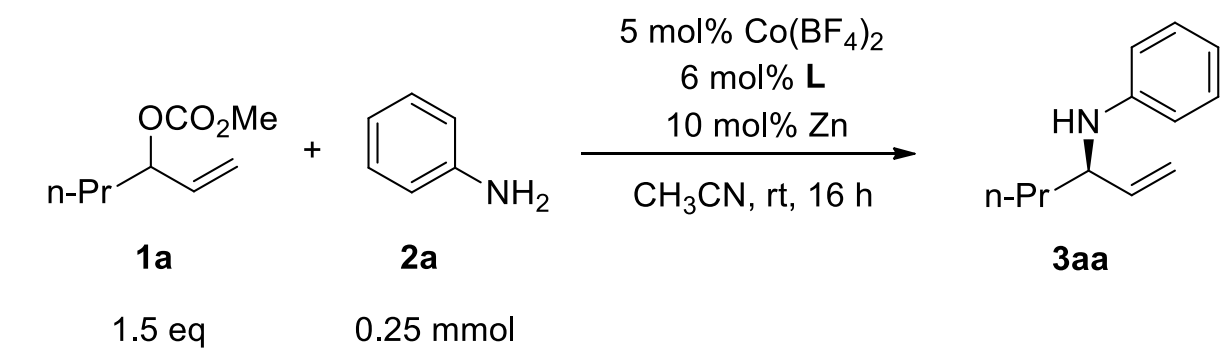

\begin{tabular}{cccc}
\hline entry & ligand & Yield (\%) & ee (\%) \\
\hline 1. & L1 & $<5$ & - \\
2. & L2 & 57 & $>99$ \\
3. & L3 & $<5$ & - \\
4. & L4 & 84 & $>99$ \\
5. & $\mathbf{L 5}$ & 68 & $>99$ \\
6. & $\mathbf{L 6}$ & $\mathbf{9 9}$ & $>99$ \\
7. & $\mathbf{L 7}$ & 38 & $>99$ \\
8. & $\mathbf{L 8}$ & 70 & $>99$ \\
9. & $\mathbf{L 9}$ & 63 & $>99$ \\
10. & $\mathbf{L 1 0}$ & 96 & $>99$ \\
11. & $\mathbf{L 1 1}$ & 96 & $>99$ \\
12. & $\mathbf{L 1 2}$ & $<5$ & - \\
13. & $\mathbf{L 1 3}$ & $<5$ & - \\
\hline
\end{tabular}<smiles>CC(C)C1COC(c2cccc(C3=N[C@@H](C(C)C)CO3)n2)=N1</smiles>

L1<smiles>[R]C1COC(c2ccccc2P([R7])c2ccccc2C2=NC([R])CO2)=N1</smiles>

L2 to L11<smiles>CC(C)C1COC(c2ccccc2Pc2ccccc2)=N1</smiles>

L12<smiles>CC(C)[C@H]1COC(CCP(CCC2=N[C@@H](C(C)C)CO2)c2ccccc2)=N1</smiles>

L13 
5. Optimization for cobalt salts and solvents with L6:

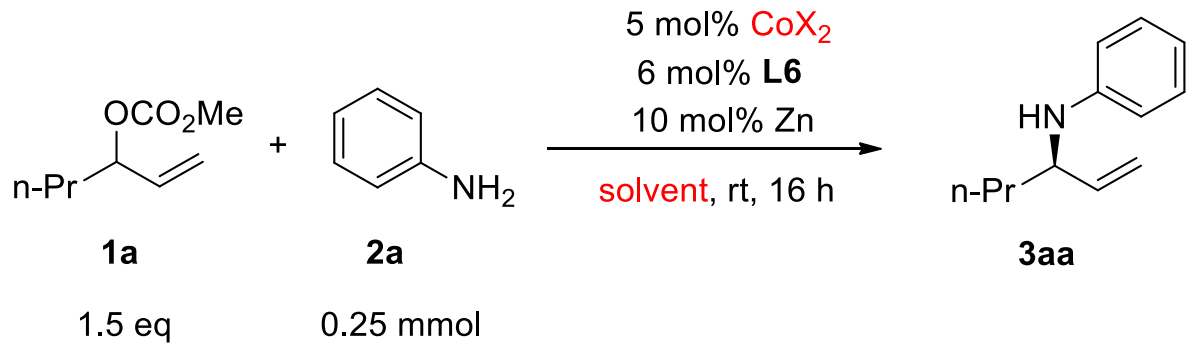

\begin{tabular}{|c|c|c|c|c|}
\hline Entry & Co-salt & Solvent & Yield (\%) & $e e(\%)$ \\
\hline 1. & $\mathrm{Co}\left(\mathrm{BF}_{4}\right)_{2}$ & $\mathrm{CH}_{3} \mathrm{CN}$ & 99 & $>99$ \\
\hline 2. & $\mathrm{Co}\left(\mathrm{BF}_{4}\right)_{2}$ & Toluene & -- & -- \\
\hline 3. & $\mathrm{Co}\left(\mathrm{BF}_{4}\right)_{2}$ & Ether & -- & -- \\
\hline 4. & $\mathrm{Co}\left(\mathrm{BF}_{4}\right)_{2}$ & THF & -- & -- \\
\hline 5. & $\mathrm{Co}\left(\mathrm{BF}_{4}\right)_{2}$ & DCM & $<5$ & -- \\
\hline 6. & $\mathrm{Co}\left(\mathrm{BF}_{4}\right)_{2}$ & DCE & $<5$ & -- \\
\hline 7. & $\mathrm{Co}\left(\mathrm{BF}_{4}\right)_{2}$ & DMF & -- & -- \\
\hline 8. & $\mathrm{Co}\left(\mathrm{BF}_{4}\right)_{2}$ & $\mathrm{MeOH}$ & $<5$ & -- \\
\hline 9. & $\mathrm{Co}(\mathrm{OAc})_{2}$ & $\mathrm{CH}_{3} \mathrm{CN}$ & -- & -- \\
\hline 10. & $\mathrm{CoCl}_{2}$ & $\mathrm{CH}_{3} \mathrm{CN}$ & -- & -- \\
\hline 11. & $\mathrm{CoBr}_{2}$ & $\mathrm{CH}_{3} \mathrm{CN}$ & -- & -- \\
\hline 12. & $\mathrm{Co}(\mathrm{acac})_{2}$ & $\mathrm{CH}_{3} \mathrm{CN}$ & -- & -- \\
\hline 13. & $\mathrm{Co}\left(\mathrm{H}_{2} \mathrm{O}\right)_{6} \bullet\left(\mathrm{ClO}_{4}\right)_{2}$ & $\mathrm{CH}_{3} \mathrm{CN}$ & 60 & $>99$ \\
\hline
\end{tabular}




\section{Representative procedure of Co-catalyzed AAA Reaction and spectral data:}

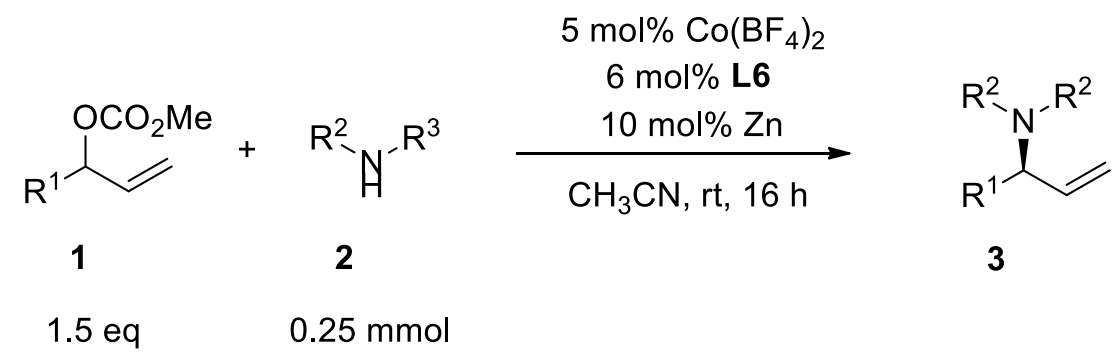

In an $\mathrm{N}_{2}$ filled glove box, $\mathrm{Zn}$ dust $\left(1.7 \mathrm{mg}, 2.6 \times 10^{-2} \mathrm{mmol}, 10 \mathrm{~mol} \%\right)$ was added to a premixed solution of 6 mol\% L6 $\left(8.3 \mathrm{mg}, 1.5 \times 10^{-2} \mathrm{mmol}\right)$ and $5 \mathrm{~mol} \% \mathrm{Co}\left(\mathrm{BF}_{4}\right)_{2}\left(2.9 \mathrm{mg}, 1.2 \times 10^{-2} \mathrm{mmol}\right)$ in $2 \mathrm{~mL}$ of acetonitrile and the mixture was stirred for 20 minutes. Then, 1 ( $0.38 \mathrm{mmol}, 1.5 \mathrm{eq})$ and $\mathbf{2}(0.25 \mathrm{mmol}, 1 \mathrm{eq})$ were added, sequentially. The reaction tube was sealed and brought out from the glove box. The reaction mixture was stirred for 16 hours at room temperature. The solvent was removed and the residue was purified by flash column chromatography with $25 \%$ toluene/PE as the eluent.

\section{$(R)-N$-(hex-1-en-3-yl)aniline (3aa): ${ }^{4}$}

Pale yellow oil; Yield: $43.4 \mathrm{mg}, 99 \%$.

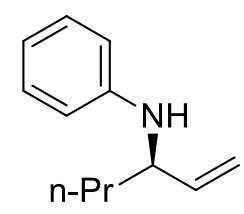

TLC $\mathbf{R}_{f}=0.3$ (PE : toluene, 3:1).

${ }^{1} \mathbf{H}$ NMR $\left(500 \mathrm{MHz}, \mathrm{CDCl}_{3}\right) \delta 7.15(\mathrm{dd}, J=8.5,7.4 \mathrm{~Hz}, 2 \mathrm{H}), 6.67(\mathrm{t}, J=7.3 \mathrm{~Hz}, 1 \mathrm{H})$, $6.60(\mathrm{dd}, J=8.5,0.9 \mathrm{~Hz}, 2 \mathrm{H}), 5.74(\mathrm{ddd}, J=16.7,10.3,6.2 \mathrm{~Hz}, 1 \mathrm{H}), 5.16(\mathrm{dd}, J=47.3$, $13.8 \mathrm{~Hz}, 2 \mathrm{H}), 3.82(\mathrm{q}, J=6.5 \mathrm{~Hz}, 1 \mathrm{H}), 3.61(\mathrm{~s}, 1 \mathrm{H}), 1.58(\mathrm{dt}, J=14.4,7.1 \mathrm{~Hz}, 3 \mathrm{H}), 1.50$ $-1.38(\mathrm{~m}, 2 \mathrm{H}), 0.95(\mathrm{t}, J=7.3 \mathrm{~Hz}, 3 \mathrm{H})$.

${ }^{13}$ C NMR $\left(101 \mathrm{MHz}, \mathrm{CDCl}_{3}\right) \delta 147.8,140.4,129.3,117.3,115.1,113.5,55.9,38.2,19.3,14.2$.

HPLC (Shimadzu LC-2030) (Daicel Chiralpak OD-H Column, ${ }^{\mathrm{i}} \mathrm{PrOH}: n-\mathrm{Hexane}=0.1: 99.9,1.0 \mathrm{ml} / \mathrm{min}$ ), R $t=$ $7.50 \mathrm{~min}$ (minor) and $8.51 \mathrm{~min}$ (major), >99\% ee.

$[\alpha]_{D}^{25}=+8.5\left(c 1.0, \mathrm{CHCl}_{3}\right)$.

HRMS (ESI): calculated $m / z$ for $\mathrm{C}_{12} \mathrm{H}_{17} \mathrm{~N}[\mathrm{M}+\mathrm{H}]^{+}=176.1434$, found 176.1440. 


\section{(R)-N-(hex-1-en-3-yl)-4-methoxyaniline (3ab): ${ }^{4}$}

Pale yellow oil; Yield: $50.3 \mathrm{mg}, 98 \%$.

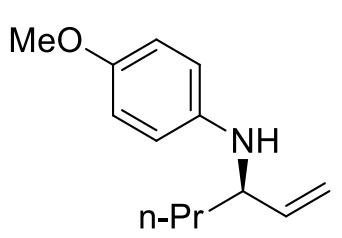

TLC $\mathbf{R}_{f}=0.3$ (PE:EA, 10:1).

${ }^{1} \mathbf{H}$ NMR $\left(400 \mathrm{MHz}, \mathrm{CDCl}_{3}\right) \delta 6.76(\mathrm{dd}, J=8.8,1.7 \mathrm{~Hz}, 2 \mathrm{H}), 6.57(\mathrm{dd}, J=8.8$, $1.5 \mathrm{~Hz}, 2 \mathrm{H}), 5.72(\mathrm{ddd}, J=16.9,10.3,6.4 \mathrm{~Hz}, 1 \mathrm{H}), 5.19(\mathrm{dd}, J=17.2,1.3 \mathrm{~Hz}$, $1 \mathrm{H}), 5.11(\mathrm{dd}, J=10.3,1.1 \mathrm{~Hz}, 1 \mathrm{H}), 3.76-3.71(\mathrm{~m}, 4 \mathrm{H}), 3.32(\mathrm{~s}, 1 \mathrm{H}), 1.56(\mathrm{tt}, J$ $=7.7,3.9 \mathrm{~Hz}, 2 \mathrm{H}), 1.50-1.40(\mathrm{~m}, 2 \mathrm{H}), 0.95(\mathrm{t}, J=7.2 \mathrm{~Hz}, 3 \mathrm{H})$.

${ }^{13} \mathbf{C}$ NMR $\left(101 \mathrm{MHz}, \mathrm{CDCl}_{3}\right) \delta 152.1,142.1,140.8,115.1,115.0,114.9,56.9,56.0,38.3,19.3,14.2$.

HPLC (Shimadzu LC-16) (Daicel Chiralpak OJ-H Column, ${ }^{\mathrm{i}} \operatorname{PrOH}: n-\mathrm{Hexane}=0.5: 99.5,1.0 \mathrm{ml} / \mathrm{min}$ ), R $t=$ $18.81 \mathrm{~min}$ (minor) and $21.18 \mathrm{~min}$ (major), $>99 \%$ ee.

$[\alpha]_{D}^{25}=-4.3\left(c 1.0, \mathrm{CHCl}_{3}\right)$

HRMS (ESI): calculated $m / z$ for $\mathrm{C}_{13} \mathrm{H}_{19} \mathrm{NO}[\mathrm{M}+\mathrm{H}]^{+}=206.1539$, found 206.1542 .

\section{(R)-4-(tert-butyl)- $N$-(hex-1-en-3-yl)aniline (3ac):}

Clear oil; Yield: $56.0 \mathrm{mg}, 97 \%$.

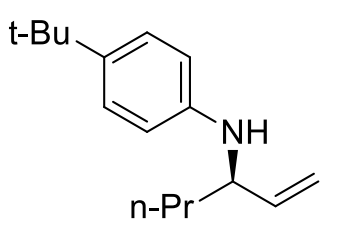

TLC $\mathbf{R}_{f}=0.3$ (PE:Toluene, 5:1).

${ }^{1} \mathbf{H}$ NMR $\left(500 \mathrm{MHz}, \mathrm{CDCl}_{3}\right) \delta 7.18(\mathrm{~d}, J=8.4 \mathrm{~Hz}, 2 \mathrm{H}), 6.56(\mathrm{~d}, J=8.4 \mathrm{~Hz}, 2 \mathrm{H})$, $5.74(\mathrm{ddd}, J=16.8,10.3,6.2 \mathrm{~Hz}, 1 \mathrm{H}), 5.22(\mathrm{~d}, J=17.2 \mathrm{~Hz}, 1 \mathrm{H}), 5.11(\mathrm{~d}, J=10.3$ $\mathrm{Hz}, 1 \mathrm{H}), 3.78(\mathrm{q}, J=6.4 \mathrm{~Hz}, 1 \mathrm{H}), 3.53(\mathrm{~s}, 1 \mathrm{H}), 1.56(\mathrm{dd}, J=14.8,7.9 \mathrm{~Hz}, 2 \mathrm{H})$, $1.48-1.40(\mathrm{~m}, 2 \mathrm{H}), 1.27(\mathrm{~s}, 9 \mathrm{H}), 0.94(\mathrm{t}, J=7.3 \mathrm{~Hz}, 3 \mathrm{H})$.

${ }^{13}$ C NMR $\left(101 \mathrm{MHz}, \mathrm{CDCl}_{3}\right) \delta 145.5,140.8,139.9,126.1,115.0,113.1,56.1,38.3,34.0,31.8,19.3,14.2$.

HPLC (Shimadzu LC-2030) (Daicel Chiralpak OJ-H Column, ${ }^{\mathrm{i}} \operatorname{PrOH}: n$-Hexane $=0.1: 99.9,1.0 \mathrm{ml} / \mathrm{min}$ ), Rt $=$ $7.13 \mathrm{~min}$ (minor) and $8.63 \mathrm{~min}$ (major), >99\% ee.

$[\alpha]_{D}^{25}=+4.3\left(c=1.0, \mathrm{CHCl}_{3}\right)$

HRMS (ESI): calculated $m / z$ for $\mathrm{C}_{16} \mathrm{H}_{25} \mathrm{~N}[\mathrm{M}+\mathrm{H}]^{+}=232.2060$, found 232.2058. 


\section{(R)-4-bromo- $N$-(hex-1-en-3-yl)aniline (3ad):}

Pale yellow oil; Yield: $60.3 \mathrm{mg}, 95 \%$.

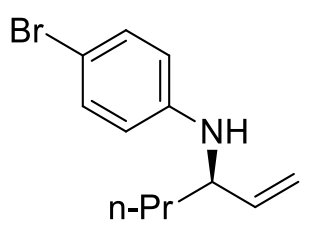

TLC $\mathbf{R}_{f}=0.3$ (PE : Toluene, 85:15).

${ }^{1} \mathbf{H}$ NMR $\left(400 \mathrm{MHz}, \mathrm{CDCl}_{3}\right) \delta 7.25-7.18(\mathrm{~m}, 2 \mathrm{H}), 6.50-6.43(\mathrm{~m}, 2 \mathrm{H}), 5.74-$ $5.66(\mathrm{~m}, 1 \mathrm{H}), 5.18(\mathrm{~d}, J=17.2 \mathrm{~Hz}, 1 \mathrm{H}), 5.12(\mathrm{~d}, J=10.3 \mathrm{~Hz}, 1 \mathrm{H}), 3.76(\mathrm{q}, J=6.5$ $\mathrm{Hz}, 1 \mathrm{H}), 3.64(\mathrm{~s}, 1 \mathrm{H}), 1.56$ (dd, $J=15.1,7.2 \mathrm{~Hz}, 2 \mathrm{H}), 1.43$ (ddd, $J=11.5,7.2,3.5$

$\mathrm{Hz}, 2 \mathrm{H}), 0.94(\mathrm{t}, J=7.3 \mathrm{~Hz}, 3 \mathrm{H})$.

${ }^{13} \mathbf{C}$ NMR $\left(101 \mathrm{MHz}, \mathrm{CDCl}_{3}\right) \delta 146.8,139.8,132.0,115.4,115.1,108.8,56.0,38.1,19.3,14.2$.

HPLC (Shimadzu LC-16) (Daicel Chiralpak OD-H Column, ${ }^{i} \mathrm{PrOH}: n-\mathrm{Hex} a n e=0.0: 100.0,1.0 \mathrm{ml} / \mathrm{min}$ ), R $t=$ $15.14 \mathrm{~min}$ (minor) and $16.44 \mathrm{~min}$ (major), $>99 \%$ ee.

$[\alpha]_{D}^{25}=+4.7\left(c 1.0, \mathrm{CHCl}_{3}\right)$

HRMS (ESI): calculated $m / z$ for $\mathrm{C}_{12} \mathrm{H}_{16} \mathrm{BrN}[\mathrm{M}+\mathrm{H}]^{+}=254.0539$, found 254.0531.

\section{(R)-1-(3-(hex-1-en-3-ylamino)phenyl)ethanone (3ae):}

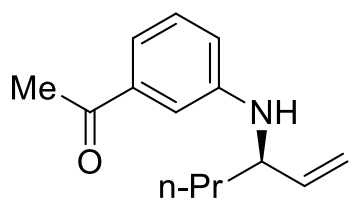

Pale yellow oil; Yield: $54.0 \mathrm{mg}, 99 \%$.

TLC $\mathbf{R}_{f}=0.8$ (PE:EA, 10:1).

${ }^{1} \mathbf{H}$ NMR $\left(400 \mathrm{MHz}, \mathrm{CDCl}_{3}\right) \delta 7.25-7.16(\mathrm{~m}, 3 \mathrm{H}), 6.77(\mathrm{dt}, J=6.9,2.3 \mathrm{~Hz}, 1 \mathrm{H})$, $5.72(\mathrm{ddd}, J=16.7,10.3,6.1 \mathrm{~Hz}, 1 \mathrm{H}), 5.21(\mathrm{~d}, J=17.2 \mathrm{~Hz}, 1 \mathrm{H}), 5.13(\mathrm{~d}, J=10.2$ $\mathrm{Hz}, 1 \mathrm{H}), 3.83(\mathrm{~d}, J=30.0 \mathrm{~Hz}, 2 \mathrm{H}), 2.55(\mathrm{~s}, 3 \mathrm{H}), 1.61-1.55(\mathrm{~m}, 2 \mathrm{H}), 1.50-1.38(\mathrm{~m}, 2 \mathrm{H}), 0.95(\mathrm{t}, J=7.3 \mathrm{~Hz}$, $3 \mathrm{H})$.

${ }^{13} \mathbf{C}$ NMR $\left(101 \mathrm{MHz}, \mathrm{CDCl}_{3}\right) \delta 198.9,148.0,139.8,138.3,129.4,118.1,117.7,115.4,112.5,55.8,38.2,26.9$, 19.3, 14.2.

HPLC (Shimadzu LC-16) (Daicel Chiralpak OJ-H Column, ${ }^{i}$ PrOH: $n$-Hexane = 1.0:99.0, $2.0 \mathrm{ml} / \mathrm{min}$ ), Rt $=$ 9.65 min (major) and 11.76 min (minor), >99\% ee.

$[\alpha]_{D}^{25}=+22.2\left(c 1.0, \mathrm{CHCl}_{3}\right)$.

HRMS (ESI): calculated $m / z$ for $\mathrm{C}_{14} \mathrm{H}_{19} \mathrm{NO}[\mathrm{M}+\mathrm{Na}]^{+}=240.1364$, found 240.1362 . 


\section{(R)- $N$-(hex-1-en-3-yl)-2-methylaniline (3af):}

Pale yellow oil; Yield: $43.0 \mathrm{mg}, 91 \%$.

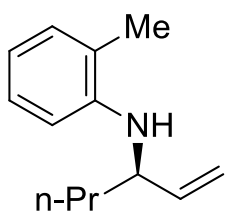

TLC $\mathbf{R}_{f}=0.5$ (PE:EA, 100:0).

${ }^{1} \mathbf{H}$ NMR $\left(400 \mathrm{MHz}, \mathrm{CDCl}_{3}\right) \delta 7.09(\mathrm{dd}, J=17.8,7.9 \mathrm{~Hz}, 2 \mathrm{H}), 6.64(\mathrm{dd}, J=15.0,7.7$ $\mathrm{Hz}, 2 \mathrm{H}), 5.79$ (ddd, $J=16.4,10.3,6.1 \mathrm{~Hz}, 1 \mathrm{H}), 5.22$ (dd, $J=17.2,1.3 \mathrm{~Hz}, 1 \mathrm{H}), 5.14$ (dd, $J=10.3,1.1 \mathrm{~Hz}, 1 \mathrm{H}), 3.89(\mathrm{q}, J=6.2 \mathrm{~Hz}, 1 \mathrm{H}), 3.49(\mathrm{~s}, 1 \mathrm{H}), 2.18(\mathrm{~s}, 3 \mathrm{H}), 1.65(\mathrm{dd}, J=$ $14.2,7.0 \mathrm{~Hz}, 2 \mathrm{H}), 1.55-1.43(\mathrm{~m}, 2 \mathrm{H}), 0.98(\mathrm{td}, J=7.3,1.7 \mathrm{~Hz}, 3 \mathrm{H})$.

${ }^{13}$ C NMR $\left(101 \mathrm{MHz}, \mathrm{CDCl}_{3}\right) \delta 145.7,140.6,130.2,127.1,121.8,116.8,115.0,110.9,55.7,38.4,19.4,17.8$, 14.2 .

HPLC (Daicel Chiralpak OJ-H Column, ${ }^{i} \mathrm{PrOH}: n$-Hexane $=0.0: 100.0,1.0 \mathrm{ml} / \mathrm{min}$ ), R $t=5.82 \mathrm{~min}$ (major) and $6.36 \mathrm{~min}$ (minor), $98 \%$ ee.

$[\alpha]_{D}^{25}=-1.1\left(c 1.0, \mathrm{CHCl}_{3}\right)$.

HRMS (ESI): calculated $m / z$ for $\mathrm{C}_{13} \mathrm{H}_{19} \mathrm{~N}[\mathrm{M}+\mathrm{H}]^{+}=190.1590$, found 190.1593 .

\section{(R)-N-(4-(hex-1-en-3-ylamino)phenyl)acetamide (3ag):}

White solid; Yield: $57.5 \mathrm{mg}, 99 \%$; M.P. $95^{\circ} \mathrm{C}$.

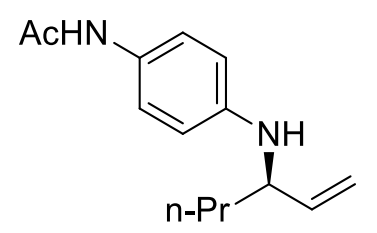

TLC $\mathbf{R}_{f}=0.5$ (PE:EA, 3:2).

${ }^{1} \mathbf{H}$ NMR $\left(500 \mathrm{MHz}, \mathrm{CDCl}_{3}\right) \delta 7.25-7.21(\mathrm{~m}, 2 \mathrm{H}), 6.56-6.53(\mathrm{~m}, 2 \mathrm{H}), 5.70$ (ddd, $J=16.8,10.3,6.2 \mathrm{~Hz}, 1 \mathrm{H}), 5.21-5.08(\mathrm{~m}, 2 \mathrm{H}), 3.76(\mathrm{dd}, J=12.9,6.5 \mathrm{~Hz}$, $1 \mathrm{H}), 2.10(\mathrm{~s}, 2.6 \mathrm{H}), 1.92(\mathrm{~s}, 0.4 \mathrm{H}), 1.55(\mathrm{dd}, J=15.6,9.4 \mathrm{~Hz}, 2 \mathrm{H}), 1.42(\mathrm{tt}, J=$ $14.2,7.1 \mathrm{~Hz}, 2 \mathrm{H}), 0.93(\mathrm{t}, J=7.3 \mathrm{~Hz}, 3 \mathrm{H})$.

${ }^{13}$ C NMR $\left(126 \mathrm{MHz}, \mathrm{CDCl}_{3}\right) \delta 168.4,144.9,140.2,128.1,122.5,115.3,113.8,56.3,38.1,24.4,19.3,14.2$.

HPLC (Shimadzu LC-16) (Daicel Chiralpak OJ-H Column, ${ }^{i} \mathrm{PrOH}: n-\mathrm{Hexane}=13: 87,1.0 \mathrm{ml} / \mathrm{min}$ ), R $t=16.12$ $\min$ (major) and $17.01 \mathrm{~min}$ (minor), $>99 \%$ ee.

$[\alpha]_{D}^{25}=-1.0\left(c 0.5, \mathrm{CHCl}_{3}\right)$.

HRMS (ESI): calculated $m / z$ for $\mathrm{C}_{14} \mathrm{H}_{20} \mathrm{~N}_{2} \mathrm{O}[\mathrm{M}+\mathrm{Na}]^{+}=255.1473$, found 255.1477. 


\section{$(R)-N$-(hex-1-en-3-yl)-1H-indol-5-amine (3ah):}

Pale yellow liquid; Yield: $53.0 \mathrm{mg}, 99 \%$.

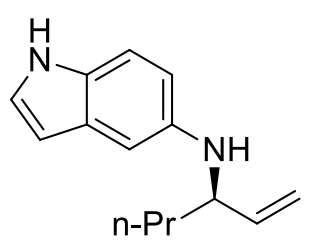

TLC $\mathbf{R}_{f}=0$. (PE:EA, 4:1).

${ }^{1} \mathbf{H}$ NMR $\left(400 \mathrm{MHz}, \mathrm{CDCl}_{3}\right) \delta 7.85(\mathrm{~s}, 1 \mathrm{H}), 7.18(\mathrm{~d}, J=8.6 \mathrm{~Hz}, 1 \mathrm{H}), 7.09(\mathrm{t}, J=2.8$ $\mathrm{Hz}, 1 \mathrm{H}), 6.86(\mathrm{~d}, J=2.0 \mathrm{~Hz}, 1 \mathrm{H}), 6.64(\mathrm{dd}, J=8.6,2.2 \mathrm{~Hz}, 1 \mathrm{H}), 6.39(\mathrm{~s}, 1 \mathrm{H}), 5.80$ (ddd, $J=16.9,10.3,6.4 \mathrm{~Hz}, 1 \mathrm{H}), 5.25(\mathrm{~d}, J=17.2 \mathrm{~Hz}, 1 \mathrm{H}), 5.12(\mathrm{~d}, J=10.3 \mathrm{~Hz}$, $1 \mathrm{H}), 3.85(\mathrm{q}, J=6.5 \mathrm{~Hz}, 1 \mathrm{H}), 1.68-1.53(\mathrm{~m}, 2 \mathrm{H}), 1.52-1.43(\mathrm{~m}, 2 \mathrm{H}), 0.97(\mathrm{t}, J=7.3 \mathrm{~Hz}, 3 \mathrm{H})$.

${ }^{13}$ C NMR $\left(126 \mathrm{MHz}, \mathrm{CDCl}_{3}\right) \delta 141.6,141.0,130.3,128.9,124.6,115.2,113.0,111.7,103.8,102.0,57.6$, $38.2,19.4,14.3$.

HPLC (Shimadzu LC-16) (Daicel Chiralpak OD-H Column, ${ }^{i} \mathrm{PrOH}: n$-Hex ane $=10: 90,2.0 \mathrm{ml} / \mathrm{min}$ ), R $t=9.37$ $\min$ (minor) and $11.71 \mathrm{~min}$ (major), $98 \%$ ee.

$[\alpha]_{D}^{25}=+8.6\left(c 0.5, \mathrm{CHCl}_{3}\right)$

HRMS (ESI): calculated $m / z$ for $\mathrm{C}_{14} \mathrm{H}_{18} \mathrm{~N}_{2}[\mathrm{M}+\mathrm{H}]^{+}=215.1542$, found 215.1544 .

\section{(R)-N-(hex-1-en-3-yl)-N-methylaniline (3ai):}

Pale yellow oil; Yield: $31.2 \mathrm{mg}, 66 \%$ (10\% catalyst).

TLC $\mathbf{R}_{f}=0.3$ (PE:Toluene, 4:1).

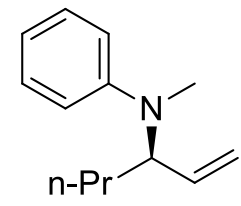

${ }^{1} \mathbf{H}$ NMR $\left(400 \mathrm{MHz}, \mathrm{CDCl}_{3}\right) \delta 7.21(\mathrm{dd}, J=11.9,4.1 \mathrm{~Hz}, 2 \mathrm{H}), 6.77(\mathrm{~d}, J=8.5 \mathrm{~Hz}, 2 \mathrm{H})$, $6.67(\mathrm{t}, J=7.2 \mathrm{~Hz}, 1 \mathrm{H}), 5.81$ (ddd, $J=15.9,10.6,4.9 \mathrm{~Hz}, 1 \mathrm{H}), 5.11$ (ddd, $J=13.9,9.4,1.2$ $\mathrm{Hz}, 2 \mathrm{H}), 4.29(\mathrm{dd}, J=13.1,5.5 \mathrm{~Hz}, 1 \mathrm{H}), 2.74(\mathrm{~s}, 3 \mathrm{H}), 1.65(\mathrm{td}, J=22.9,11.5 \mathrm{~Hz}, 2 \mathrm{H})$, $1.34(\mathrm{dd}, J=21.2,7.0 \mathrm{~Hz}, 2 \mathrm{H}), 0.91(\mathrm{t}, J=7.4 \mathrm{~Hz}, 3 \mathrm{H})$.

${ }^{13}$ C NMR $\left(126 \mathrm{MHz}, \mathrm{CDCl}_{3}\right) \delta 150.8,137.8,129.3,116.5,115.5,113.1,60.0,34.4,31.5,20.1,14.3$.

HPLC (Shimadzu LC-2030) (Daicel Chiralpak OD-H Column, ${ }^{i} \mathrm{PrOH}: n-\mathrm{Hex} a n e=0.0: 100.0,0.3 \mathrm{ml} / \mathrm{min}$ ), R $t$ $=28.25 \mathrm{~min}$ (minor) and $30.46 \mathrm{~min}$ (major), $99 \%$ ee.

$[\alpha]_{D}^{25}=+109.3\left(c 1.0, \mathrm{CHCl}_{3}\right)$.

HRMS (ESI): calculated $\mathrm{m} / z$ for $\mathrm{C}_{13} \mathrm{H}_{19} \mathrm{~N}[\mathrm{M}+\mathrm{H}]^{+}=190.1590$, found 190.1595 . 


\section{(R)-1-(hex-1-en-3-yl)indoline (3aj):}

Pale yellow oil; Yield: $49.8 \mathrm{mg}, 99 \%$.

TLC $\mathbf{R}_{f}=0.2$ (PE:Toluene, 4:1).

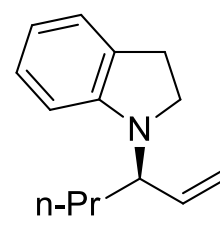

${ }^{1} \mathbf{H}$ NMR $\left(400 \mathrm{MHz}, \mathrm{CDCl}_{3}\right) \delta 7.04(\mathrm{t}, J=7.8 \mathrm{~Hz}, 2 \mathrm{H}), 6.60(\mathrm{t}, J=7.3 \mathrm{~Hz}, 1 \mathrm{H}), 6.44(\mathrm{~d}, J=$ $7.8 \mathrm{~Hz}, 1 \mathrm{H}), 5.84-5.75(\mathrm{~m}, 1 \mathrm{H}), 5.18(\mathrm{dd}, J=15.3,3.6 \mathrm{~Hz}, 2 \mathrm{H}), 3.98(\mathrm{q}, J=7.0 \mathrm{~Hz}, 1 \mathrm{H})$, $3.46-3.33(\mathrm{~m}, 2 \mathrm{H}), 2.96(\mathrm{t}, J=8.5 \mathrm{~Hz}, 2 \mathrm{H}), 1.77-1.68(\mathrm{~m}, 1 \mathrm{H}), 1.63-1.58(\mathrm{~m}, 1 \mathrm{H}), 1.50$ $-1.39(\mathrm{~m}, 2 \mathrm{H}), 0.97(\mathrm{dd}, J=11.9,4.5 \mathrm{~Hz}, 3 \mathrm{H})$.

${ }^{13}$ C NMR $\left(101 \mathrm{MHz}, \mathrm{CDCl}_{3}\right) \delta 151.6,136.4,130.1,127.4,124.6,117.0,116.9,107.4,57.6,47.0,33.8,28.4$, 19.9, 14.2.

HPLC (Shimadzu LC-16) (Daicel Chiralpak OD-H Column, ${ }^{i} \mathrm{PrOH}: n-\mathrm{Hex} a n e=0.0: 100.0,1.0 \mathrm{ml} / \mathrm{min}$ ), R $t=$ $11.84 \mathrm{~min}$ (major) and $12.49 \mathrm{~min}$ (minor), $99 \%$ ee.

$[\alpha]_{D}^{25}=+39.7\left(c 1.0, \mathrm{CHCl}_{3}\right)$.

HRMS (ESI): calculated $m / z$ for $\mathrm{C}_{14} \mathrm{H}_{19} \mathrm{~N}[\mathrm{M}+\mathrm{H}]^{+}=202.1590$, found 202.1597.

\section{(R)-1-(hex-1-en-3-yl)-1-(4-methoxyphenyl)hydrazine (3ak):}

Pale yellow oil; Yield: $39.5 \mathrm{mg}, 83 \%$.

TLC $\mathbf{R}_{f}=0.7$ (PE:EA, 10:1).

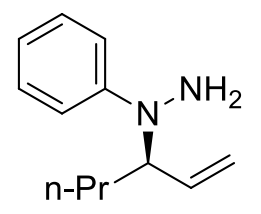

${ }^{1} \mathbf{H}$ NMR $\left(500 \mathrm{MHz}, \mathrm{CDCl}_{3}\right) \delta 7.23(\mathrm{~d}, J=7.4 \mathrm{~Hz}, 2 \mathrm{H}), 7.04(\mathrm{~d}, J=8.3 \mathrm{~Hz}, 2 \mathrm{H}), 6.76(\mathrm{t}$, $J=7.2 \mathrm{~Hz}, 1 \mathrm{H}), 5.80(\mathrm{ddd}, J=16.7,10.6,5.8 \mathrm{~Hz}, 1 \mathrm{H}), 5.20(\mathrm{~d}, \mathrm{~J}=10.6 \mathrm{~Hz}, 1 \mathrm{H}), 5.13(\mathrm{~d}$, $J=17.5 \mathrm{~Hz}, 1 \mathrm{H}), 4.28(\mathrm{dd}, J=13.8,6.5 \mathrm{~Hz}, 1 \mathrm{H}), 3.29(\mathrm{~s}, 2 \mathrm{H}), 1.85-1.78(\mathrm{~m}, 1 \mathrm{H}), 1.66$ $-1.59(\mathrm{~m}, 1 \mathrm{H}), 1.44-1.35(\mathrm{~m}, 2 \mathrm{H}), 0.95(\mathrm{t}, J=7.4 \mathrm{~Hz}, 3 \mathrm{H})$.

${ }^{13}$ C NMR $\left(101 \mathrm{MHz}, \mathrm{CDCl}_{3}\right) \delta 152.1,135.9,129.2,118.4,117.3,114.0,62.1,32.9,19.9,14.3$.

HPLC (Shimadzu LC-16) (Daicel Chiralpak OJ-H Column, ${ }^{i} \mathrm{PrOH}: n$-Hexane $=0: 100,0.5 \mathrm{ml} / \mathrm{min}$ ), R $t=38.22$ min (minor) and 40.05 min (major), $>99 \%$ ee.

$[\alpha]_{D}^{25}=+103.2\left(c 0.5, \mathrm{CHCl}_{3}\right)$.

HRMS (ESI): calculated $m / z$ for $\mathrm{C}_{12} \mathrm{H}_{18} \mathrm{~N}_{2}[\mathrm{M}+\mathrm{H}]^{+}=191.1543$, found 191.1547. 


\section{(R)- $N$-(but-3-en-2-yl)-4-methoxyaniline (3bb): ${ }^{5}$}

Pale yellow oil; Yield: $37.2 \mathrm{mg}, 84 \%$.

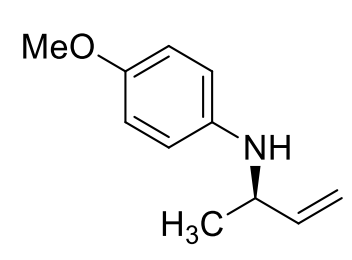

TLC $\mathbf{R}_{f}=0.5$ (PE:EA, 9:1).

${ }^{1} \mathbf{H}$ NMR $\left(400 \mathrm{MHz}, \mathrm{CDCl}_{3}\right) \delta 6.76(\mathrm{dd}, J=9.6,2.8 \mathrm{~Hz}, 2 \mathrm{H}), 6.69-6.57(\mathrm{~m}, 2 \mathrm{H})$, $5.85-5.78(\mathrm{~m}, 1 \mathrm{H}), 5.20(\mathrm{~d}, J=17.2 \mathrm{~Hz}, 1 \mathrm{H}), 5.07(\mathrm{~d}, J=10.3 \mathrm{~Hz}, 1 \mathrm{H}), 3.91(\mathrm{p}, J$ $=6.6 \mathrm{~Hz}, 1 \mathrm{H}), 3.74(\mathrm{~s}, 3 \mathrm{H}), 1.29(\mathrm{~d}, J=6.6 \mathrm{~Hz}, 3 \mathrm{H})$.

${ }^{13}$ C NMR (101 MHz, $\left.\mathrm{CDCl}_{3}\right) \delta 152.2,141.9,141.8,115.1,115.0,114.3,56.0,52.3,21.9$.

HPLC (Shimadzu LC-2030) (Daicel Chiralpak OJ-H Column, ${ }^{i} \operatorname{PrOH}: n-\mathrm{Hexane}=0.5: 99.5,2.0 \mathrm{ml} / \mathrm{min}$ ), R $t=$ $28.66 \mathrm{~min}$ (minor) and $32.37 \mathrm{~min}$ (majoro), 97\% ee.

$[\alpha]_{D}^{25}=-6.5\left(c 1.0, \mathrm{CHCl}_{3}\right)$.

HRMS (ESI): calculated $m / z$ for $\mathrm{C}_{11} \mathrm{H}_{15} \mathrm{NO}[\mathrm{M}+\mathrm{H}]^{+}=178.1226$, found 178.1230.

\section{(R)-4-methoxy- $N$-(5-phenylpent-1-en-3-yl)aniline (3cb): ${ }^{5}$}

Pale yellow oil; Yield: $65.5 \mathrm{mg}, 98 \%$.

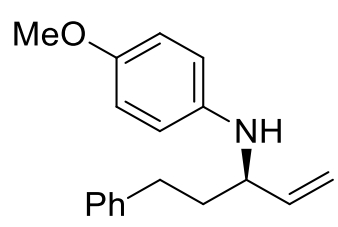

TLC $\mathbf{R}_{f}=0.6$ (PE:EA, 10:1).

${ }^{1} \mathbf{H}$ NMR $\left(500 \mathrm{MHz}, \mathrm{CDCl}_{3}\right) \delta 7.29(\mathrm{t}, J=7.4 \mathrm{~Hz}, 2 \mathrm{H}), 7.20(\mathrm{t}, J=7.6 \mathrm{~Hz}, 3 \mathrm{H})$, $6.76(\mathrm{~d}, J=8.8 \mathrm{~Hz}, 2 \mathrm{H}), 6.54(\mathrm{~d}, J=8.8 \mathrm{~Hz}, 2 \mathrm{H}), 5.77$ (ddd, $J=16.7,10.3,6.0$ $\mathrm{Hz}, 1 \mathrm{H}), 5.21(\mathrm{dd}, J=17.2,1.1 \mathrm{~Hz}, 1 \mathrm{H}), 5.15(\mathrm{dd}, J=10.3,1.0 \mathrm{~Hz}, 1 \mathrm{H}), 3.79-$ $3.74(\mathrm{~m}, 1 \mathrm{H}), 3.74(\mathrm{~d}, J=0.6 \mathrm{~Hz}, 3 \mathrm{H}), 2.75(\mathrm{t}, J=7.8 \mathrm{~Hz}, 2 \mathrm{H}), 1.91(\mathrm{dt}, J=11.7,7.1 \mathrm{~Hz}, 2 \mathrm{H})$.

${ }^{13}$ C NMR (126 MHz, $\left.\mathrm{CDCl}_{3}\right) \delta 152.2,141.9,141.7,140.3,128.7,128.6,126.1,115.7,115.1,115.0,56.6$, $56.0,37.5,32.4$.

HPLC (Shimadzu LC-16) (Daicel Chiralpak OJ-H Column, ${ }^{i} \mathrm{PrOH}: n$-Hexane $=0.5: 99.5,2.0 \mathrm{ml} / \mathrm{min}$ ), R $t=$ $22.91 \mathrm{~min}$ (major) and $26.66 \mathrm{~min}$ (minor), >99\% ee.

$[\alpha]_{D}^{25}=-10.7\left(c 1.0, \mathrm{CHCl}_{3}\right)$.

HRMS (ESI): calculated $m / z$ for $\mathrm{C}_{18} \mathrm{H}_{21} \mathrm{NO}[\mathrm{M}+\mathrm{H}]^{+}=268.1696$, found 268.1701. 


\section{(R)-4-methoxy- $N$-(4-methylpent-1-en-3-yl)aniline (3db): ${ }^{6}$}

Pale yellow oil; Yield: $45.7 \mathrm{mg}, 89 \%$.

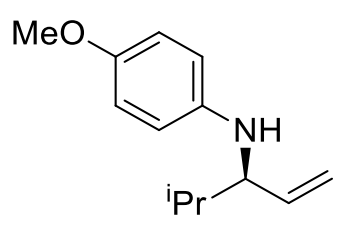

TLC $\mathbf{R}_{f}=0.7$ (PE:EA, 20:1).

${ }^{1} \mathbf{H}$ NMR $\left(500 \mathrm{MHz}, \mathrm{CDCl}_{3}\right) \delta 6.78-6.74(\mathrm{~m}, 2 \mathrm{H}), 6.60-6.57(\mathrm{~m}, 2 \mathrm{H}), 5.71$

(ddd, $J=17.0,10.4,6.5 \mathrm{~Hz}, 1 \mathrm{H}), 5.16$ (ddt, $J=10.4,4.6,1.6 \mathrm{~Hz}, 2 \mathrm{H}), 3.74$ (s,

$3 \mathrm{H}), 3.56(\mathrm{dd}, J=6.5,5.4 \mathrm{~Hz}, 1 \mathrm{H}), 1.86(\mathrm{dq}, J=13.6,6.8 \mathrm{~Hz}, 1 \mathrm{H}), 0.99(\mathrm{~d}, J=$

$6.8 \mathrm{~Hz}, 3 \mathrm{H}), 0.96(\mathrm{~d}, J=6.8 \mathrm{~Hz}, 3 \mathrm{H})$.

${ }^{13} \mathbf{C ~ N M R}\left(126 \mathrm{MHz}, \mathrm{CDCl}_{3}\right) \delta 152.1,142.2,138.3,116.3,115.0,62.8,56.0,32.6,19.1,18.7$.

HPLC (Shimadzu LC-16) (Daicel Chiralpak OJ-H Column, ${ }^{i} \mathrm{PrOH}: n$-Hexane $=0.5: 95.5,2.0 \mathrm{ml} / \mathrm{min}$ ), Rt $=$ $11.19 \mathrm{~min}$ (minor) and $13.12 \mathrm{~min}$ (major), >99\% ee.

$[\alpha]_{D}^{25}=-2.2\left(c 0.5, \mathrm{CHCl}_{3}\right)$.

HRMS (ESI): calculated $m / z$ for $\mathrm{C}_{13} \mathrm{H}_{19} \mathrm{NO}[\mathrm{M}+\mathrm{H}]^{+}=206.1539$, found 206.1540.

(S)-N-(1-cyclohexylallyl)-4-methoxyaniline (3eb): ${ }^{7}$

Pale yellow oil; Yield: $49.7 \mathrm{mg}, 81 \%$.

TLC $\mathbf{R}_{f}=0.7$ (PE:EA, 20:1).

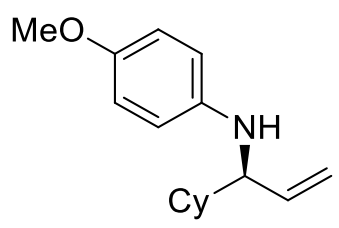

${ }^{1} \mathbf{H}$ NMR $\left(400 \mathrm{MHz}, \mathrm{CDCl}_{3}\right) \delta 6.78-6.74(\mathrm{~m}, 2 \mathrm{H}), 6.58-6.54(\mathrm{~m}, 2 \mathrm{H}), 5.70(\mathrm{ddd}$, $J=17.1,10.4,6.7 \mathrm{~Hz}, 1 \mathrm{H}), 5.17-5.13(\mathrm{~m}, 2 \mathrm{H}), 3.74(\mathrm{~s}, 3 \mathrm{H}), 3.56(\mathrm{t}, J=6.1 \mathrm{~Hz}$, $1 \mathrm{H}), 3.44(\mathrm{~s}, 1 \mathrm{H}), 1.87-1.67(\mathrm{~m}, 5 \mathrm{H}), 1.53-1.45(\mathrm{~m}, 1 \mathrm{H}), 1.30-1.03(\mathrm{~m}, 5 \mathrm{H})$.

${ }^{13} \mathbf{C}$ NMR $\left(126 \mathrm{MHz}, \mathrm{CDCl}_{3}\right) \delta 152.0,142.4,138.9,116.0,115.0,114.8,62.3,56.0$, $42.9,29.7,29.5,26.8,26.6,26.5$.

HPLC (Shimadzu LC-16) (Daicel Chiralpak OJ-H Column, ${ }^{i} \operatorname{PrOH}: n$-Hexane $=2.0: 98.0,2.0 \mathrm{ml} / \mathrm{min}$ ), R $t=$ $6.06 \mathrm{~min}$ (minor) and $7.68 \mathrm{~min}$ (major), >99\% ee.

$[\alpha]_{D}^{25}=-12.2\left(c 1.0, \mathrm{CHCl}_{3}\right)$.

HRMS (ESI): calculated $m / z$ for $\mathrm{C}_{16} \mathrm{H}_{23} \mathrm{NO}[\mathrm{M}+\mathrm{H}]^{+}=246.1852$, found 246.1853. 


\section{(S)-N-(1-cyclopropylallyl)-4-methoxyaniline (3fb):}

Pale yellow Liquid; Yield: $44.7 \mathrm{mg}, 88 \%$.

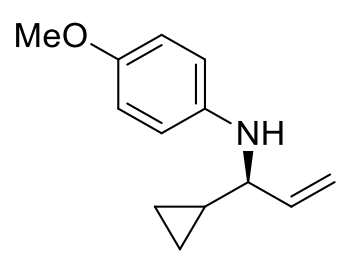

TLC $\mathbf{R}_{f}=0.7$ (PE:EA, 20:1).

${ }^{1} \mathbf{H}$ NMR $\left(500 \mathrm{MHz}, \mathrm{CDCl}_{3}\right) \delta 6.77-6.74(\mathrm{~m}, 2 \mathrm{H}), 6.60-6.57(\mathrm{~m}, 2 \mathrm{H}), 5.85-$ $5.77(\mathrm{~m}, 1 \mathrm{H}), 5.24(\mathrm{dt}, J=17.2,1.3 \mathrm{~Hz}, 1 \mathrm{H}), 5.13(\mathrm{dt}, J=10.3,1.2 \mathrm{~Hz}, 1 \mathrm{H}), 3.74$ (s, 3H), $3.15(\mathrm{dd}, J=7.5,6.4 \mathrm{~Hz}, 1 \mathrm{H}), 1.05-0.98(\mathrm{~m}, 1 \mathrm{H}), 0.57-0.53(\mathrm{~m}, 2 \mathrm{H})$, $0.36-0.34(\mathrm{~m}, 2 \mathrm{H})$.

${ }^{13} \mathbf{C}$ NMR $\left(126 \mathrm{MHz}, \mathrm{CDCl}_{3}\right) \delta 152.2,142.2,139.4,115.4,115.1,114.9,61.5,56.0,17.0,3.6,2.7$.

HPLC (Shimadzu LC-16) (Daicel Chiralpak OJ-H Column, ${ }^{i} \mathrm{PrOH}: n-\mathrm{Hexane}=0.0: 100.0,3.0 \mathrm{ml} / \mathrm{min}$ ), R $t=$ $16.54 \mathrm{~min}$ (minor) and $17.36 \mathrm{~min}$ (major), >99\% ee.

$[\alpha]_{D}^{25}=-38.7\left(c 1.0, \mathrm{CHCl}_{3}\right)$

HRMS (ESI): calculated $m / z$ for $\mathrm{C}_{11} \mathrm{H}_{15} \mathrm{NO}[\mathrm{M}+\mathrm{H}]^{+}=178.1226$, found 178.1230.

\section{(R)-4-methoxy- $N$-(5-methylhex-1-en-3-yl)aniline (3gb): ${ }^{5}$}

Pale yellow oil; Yield: $52.1 \mathrm{mg}, 95 \%$.

TLC $\mathbf{R}_{f}=0.7$ (PE:EA, 10:1).

${ }^{1} \mathbf{H}$ NMR $\left(500 \mathrm{MHz}, \mathrm{CDCl}_{3}\right) \delta 6.78-6.74(\mathrm{~m}, 2 \mathrm{H}), 6.59-6.56(\mathrm{~m}, 2 \mathrm{H}), 5.69(\mathrm{ddd}, J=17.1,10.3,6.5 \mathrm{~Hz}$, $1 \mathrm{H}), 5.19$ (d, $J=17.2 \mathrm{~Hz}, 1 \mathrm{H}), 5.09(\mathrm{~d}, J=10.3 \mathrm{~Hz}, 1 \mathrm{H}), 3.78$ (q, $J=7.0 \mathrm{~Hz}, 1 \mathrm{H}), 3.74(\mathrm{~s}, 3 \mathrm{H}), 1.78(\mathrm{dp}, J=$ $13.4,6.7 \mathrm{~Hz}, 1 \mathrm{H}), 1.47(\mathrm{dt}, J=14.3,7.2 \mathrm{~Hz}, 1 \mathrm{H}), 1.40(\mathrm{dt}, J=13.8,7.0 \mathrm{~Hz}, 1 \mathrm{H})$,

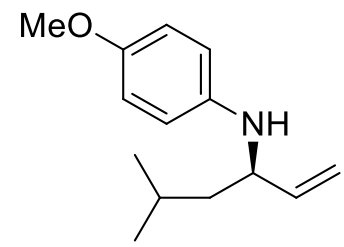
$0.96(\mathrm{~d}, J=6.6 \mathrm{~Hz}, 3 \mathrm{H}), 0.93(\mathrm{~d}, J=6.6 \mathrm{~Hz}, 3 \mathrm{H})$.

${ }^{13} \mathbf{C}$ NMR $\left(126 \mathrm{MHz}, \mathrm{CDCl}_{3}\right.$ ) $\delta 152.1,142.1,141.0,115.0$ (for 2C), 114.9, 56.0, $55.3,45.6,24.9,22.9$ (for $2 \mathrm{C}$ ).

HPLC (Shimadzu LC-16) (Daicel Chiralpak OJ-H Column, ${ }^{i} \mathrm{PrOH}: n-\mathrm{Hex}$ ane $=$ 0.5:99.5, $3.0 \mathrm{ml} / \mathrm{min}$ ), $\mathrm{R} t=5.78 \mathrm{~min}$ (minor) and $9.71 \mathrm{~min}$ (major), $>99 \%$ ee. $[\alpha]_{D}^{25}=-6.8\left(c 0.5, \mathrm{CHCl}_{3}\right)$

HRMS (ESI): calculated $m / z$ for $\mathrm{C}_{14} \mathrm{H}_{21} \mathrm{NO}[\mathrm{M}+\mathrm{H}]^{+}=220.1696$, found 220.1703. 


\section{(S)-4-methoxy- $N$-(1-phenylallyl)aniline (3hb): ${ }^{4}$}

Pale yellow oil; Yield: $50.2 \mathrm{mg}$, 84\% (using L4 ligand).

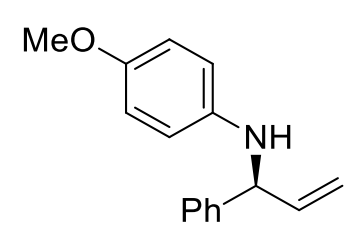

TLC $\mathbf{R}_{f}=0.6$ (PE:EA, 4:1).

${ }^{1} \mathbf{H}$ NMR $\left(500 \mathrm{MHz}, \mathrm{CDCl}_{3}\right) \delta 7.35(\mathrm{dt}, J=13.0,8.2 \mathrm{~Hz}, 4 \mathrm{H}), 7.28-7.24(\mathrm{~m}, 1 \mathrm{H})$, $6.74-6.72(\mathrm{~m}, 2 \mathrm{H}), 6.57-6.55(\mathrm{~m}, 2 \mathrm{H}), 6.06-5.99(\mathrm{~m}, 1 \mathrm{H}), 5.26(\mathrm{dt}, J=17.1$, $1.3 \mathrm{~Hz}, 1 \mathrm{H}), 5.20(\mathrm{dt}, J=10.2,1.2 \mathrm{~Hz}, 1 \mathrm{H}), 4.85(\mathrm{~d}, J=6.0 \mathrm{~Hz}, 1 \mathrm{H}), 3.71(\mathrm{~s}, 3 \mathrm{H})$.

${ }^{13} \mathbf{C}$ NMR $\left(126 \mathrm{MHz}, \mathrm{CDCl}_{3}\right) \delta 152.4,142.3,141.6,139.7,128.9,127.6,127.3,116.1,115.1,114.9,62.0$, 56.0.

HPLC (Shimadzu LC-16) (Daicel Chiralpak OJ-H Column, ${ }^{i} \mathrm{PrOH}: n$-Hexane $=1.0: 99.0,2.0 \mathrm{ml} / \mathrm{min}$ ), R $t=$ $22.69 \mathrm{~min}$ (minor) and $23.35 \mathrm{~min}$ (major), $99 \%$ ee.

$[\alpha]_{D}^{25}=-16.1\left(c 1.0, \mathrm{CHCl}_{3}\right)$.

HRMS (ESI): calculated $\mathrm{m} / z$ for $\mathrm{C}_{16} \mathrm{H}_{17} \mathrm{NO}[\mathrm{M}+\mathrm{H}]^{+}=240.1383$, found 240.1382 .

\section{(S)-2-(4-methoxyphenylamino)but-3-en-1-ol (3ib): ${ }^{8}$}

Pale yellow oil; Yield: $43.0 \mathrm{mg}, 89 \%$ (10 mol\% catalyst).

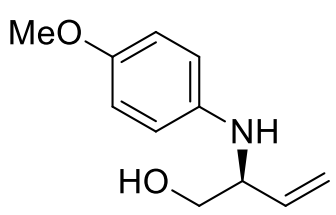

TLC $\mathbf{R}_{f}=0.3$ (PE:EA, 4:1).

${ }^{1} \mathbf{H}$ NMR $\left(400 \mathrm{MHz}, \mathrm{CDCl}_{3}\right) \delta 6.79-6.76(\mathrm{~m}, 2 \mathrm{H}), 6.68-6.65(\mathrm{~m}, 2 \mathrm{H}), 5.79$ (ddd, $J=17.1,10.4,5.8 \mathrm{~Hz}, 1 \mathrm{H}), 5.29(\mathrm{~d}, J=17.3 \mathrm{~Hz}, 1 \mathrm{H}), 5.29$ (d, $J=17.3 \mathrm{~Hz}$, $1 \mathrm{H}), 5.23(\mathrm{~d}, J=10.4 \mathrm{~Hz}, 1 \mathrm{H}), 3.94(\mathrm{dd}, J=11.1,5.8 \mathrm{~Hz}, 1 \mathrm{H}), 3.78(\mathrm{dd}, J=10.9$, $4.3 \mathrm{~Hz}, 1 \mathrm{H}), 3.74(\mathrm{~s}, 3 \mathrm{H}), 3.61(\mathrm{dd}, J=10.9,6.8 \mathrm{~Hz}, 1 \mathrm{H}), 2.67$ (s, 2H).

${ }^{13} \mathbf{C}$ NMR $\left(126 \mathrm{MHz}, \mathrm{CDCl}_{3}\right) \delta 152.9,141.4,136.8,117.6,115.9,115.0,65.1,59.1,55.9$.

HPLC (Shimadzu LC-16) (Daicel Chiralpak OJ-H Column, ${ }^{i} \operatorname{PrOH}: n-H e x a n e=7.0: 93.0,3.0 \mathrm{ml} / \mathrm{min}$ ), R $t=$ $10.18 \mathrm{~min}$ (minor) and $11.18 \mathrm{~min}$ (major), 94\% ee.

$[\alpha]_{D}^{25}=+17.8\left(c 0.5, \mathrm{CHCl}_{3}\right)$

HRMS (ESI): calculated $m / z$ for $\mathrm{C}_{11} \mathrm{H}_{15} \mathrm{NO}_{2}[\mathrm{M}+\mathrm{H}]^{+}=194.1176$, found 194.1180. 
(R)- $N$-(6-(tert-butyldimethylsilyloxy)hex-1-en-3-yl)-4-methoxyaniline (3jb): ${ }^{7}$

Pale yellow oil; Yield: $80.5 \mathrm{mg}, 96 \%$.

TLC $\mathbf{R}_{f}=0.8$ (PE:EA, 10:1).

${ }^{1} \mathbf{H}$ NMR $\left(500 \mathrm{MHz}, \mathrm{CDCl}_{3}\right) \delta 6.77-6.74(\mathrm{~m}, 2 \mathrm{H}), 6.60-6.56(\mathrm{~m}, 2 \mathrm{H}), 5.73(\mathrm{ddd}, J=16.9,10.3,6.3 \mathrm{~Hz}$, $1 \mathrm{H}), 5.20(\mathrm{~d}, J=17.2 \mathrm{~Hz}, 1 \mathrm{H}), 5.12(\mathrm{~d}, J=10.3 \mathrm{~Hz}, 1 \mathrm{H}), 3.74(\mathrm{~s}, 4 \mathrm{H}), 3.64(\mathrm{t}, J=$<smiles>C=C[C@H](CCCOC(C)(C)C)Nc1ccc(OC)cc1</smiles>
$5.6 \mathrm{~Hz}, 2 \mathrm{H}), 1.67-1.61(\mathrm{~m}, 4 \mathrm{H}), 0.90(\mathrm{~s}, 9 \mathrm{H}), 0.06(\mathrm{~s}, 6 \mathrm{H})$.

${ }^{13}$ C NMR $\left(126 \mathrm{MHz}, \mathrm{CDCl}_{3}\right) \delta 152.1,142.0,140.6,115.3,115.0,114.9,63.1$, 57.0, 56.0, 32.2, 29.4, 26.2, 18.6, -5.1 .

HPLC (Shimadzu LC-16) (Daicel Chiralpak IC-H Column, ${ }^{i} \operatorname{PrOH}: n-\mathrm{Hex}$ ane $=0.5: 99.5,1.0 \mathrm{ml} / \mathrm{min}$ ), R $t=$ $8.62 \mathrm{~min}$ (minor) and $12.75 \mathrm{~min}$ (major), >99\% ee.

$[\alpha]_{D}^{25}=-8.7\left(c 1.0, \mathrm{CHCl}_{3}\right)$

HRMS (ESI): calculated $\mathrm{m} / z$ for $\mathrm{C}_{19} \mathrm{H}_{33} \mathrm{NO}_{2} \mathrm{Si}[\mathrm{M}+\mathrm{H}]^{+}=336.2353$, found 336.2359.

\section{Optimization for linear allylic carbonate ( $E$ and $Z$ isomers):}

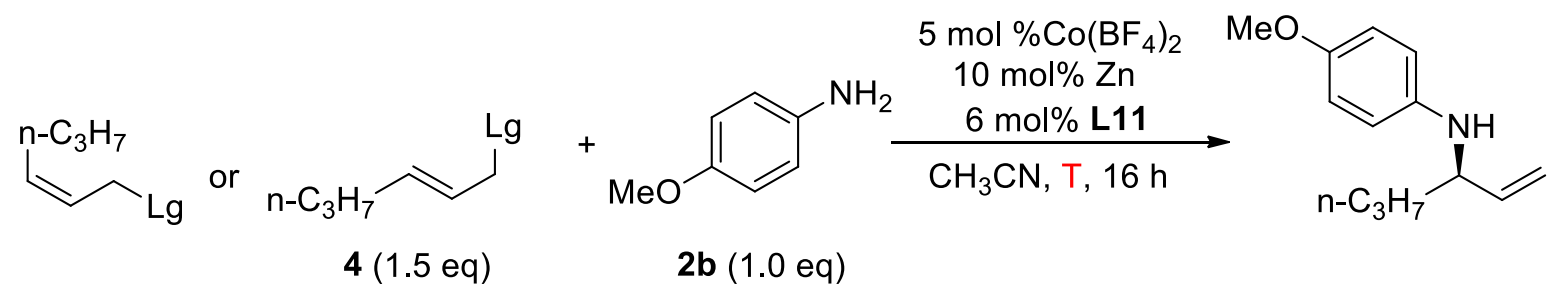

\begin{tabular}{cccccc}
\hline Entry & Carbonate & T $\left({ }^{\circ} \mathbf{C}\right)$ & Yield & B:L & e e \\
\hline 1. & $E, \mathrm{Lg}=\mathrm{OCOOMe}$ & 25 & - & - & - \\
2. & $E, \mathrm{Lg}=\mathrm{OCOOCH}\left(\mathrm{CF}_{3}\right)_{2}$ & 25 & $45 \%$ & $>50: 1$ & $99 \%$ \\
3. & $E, \mathrm{Lg}=\mathrm{OCOOCH}\left(\mathrm{CF}_{3}\right)_{2}$ & 40 & $55 \%$ & $>50: 1$ & $99 \%$ \\
4. & $E, \mathrm{Lg}=\mathrm{OCOOCH}\left(\mathrm{CF}_{3}\right)_{2}$ & 60 & $70 \%$ & $>50: 1$ & $99 \%$ \\
5. & $E, \mathrm{Lg}=\mathrm{OCOOCH}\left(\mathrm{CF}_{3}\right)_{2}$ & 80 & $<50$ & - & - \\
6. & $Z, \mathrm{Lg}=\mathrm{OCOOCH}\left(\mathrm{CF}_{3}\right)_{2}$ & 60 & $70 \%$ & $13: 1$ & $95 \%$ \\
\hline
\end{tabular}




\section{Representative procedure of Co-catalyzed AAA Reaction of aliphatic amines:}

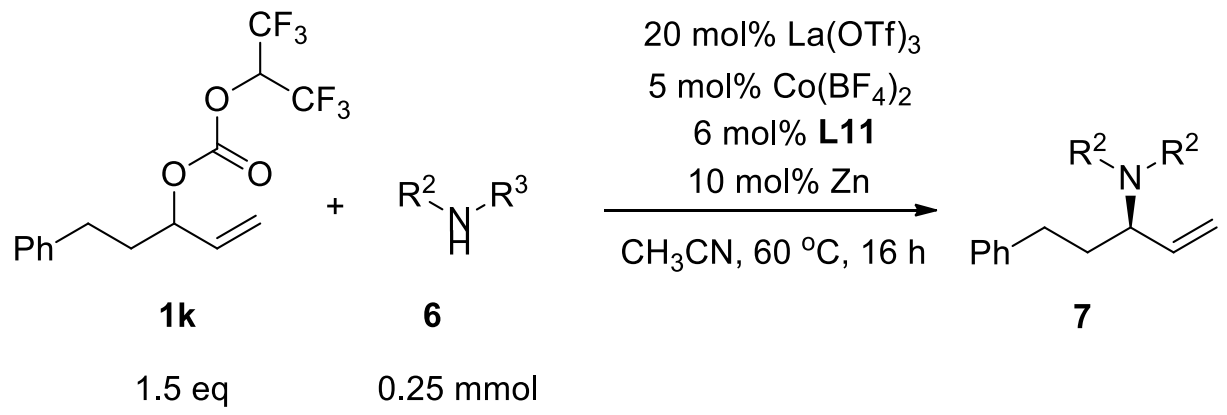

In an $\mathrm{N}_{2}$ filled glove box, $\mathrm{Zn}$ dust $\left(1.7 \mathrm{mg}, 2.6 \times 10^{-2} \mathrm{mmol}, 10 \mathrm{~mol} \%\right)$ was added to a premixed solution of 6 mol\% L11 (10.9 mg, $\left.1.5 \times 10^{-2} \mathrm{mmol}\right)$ and $5 \mathrm{~mol} \% \mathrm{Co}\left(\mathrm{BF}_{4}\right)_{2}\left(2.9 \mathrm{mg}, 1.2 \times 10^{-2} \mathrm{mmol}\right)$ in $2 \mathrm{~mL}$ of acetonitrile and the mixture was stirred for 20 minutes. Then, $\mathbf{1 k}(0.38 \mathrm{mmol}, 1.5 \mathrm{eq}), \mathrm{La}(\mathrm{OTf})_{3}\left(29.0 \mathrm{mg}, 5.0 \times 10^{-2} \mathrm{mmol}\right.$, $20 \mathrm{~mol} \%)$ and $6(0.25 \mathrm{mmol}, 1 \mathrm{eq})$ were added sequentially. The reaction tube was sealed and brought out from the glove box. The reaction mixture was stirred for 16 hours at $60{ }^{\circ} \mathrm{C}$. The solvent was removed and the residue was purified by flash column chromatography.

\section{(R)-4-(5-phenylpent-1-en-3-yl)morpholine (7ka):}

Clear oil; Yield: $57.3 \mathrm{mg}, 99 \%$.

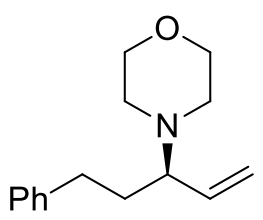

TLC $\mathbf{R}_{f}=0.2($ PE:EA, 1:1).

${ }^{1} \mathbf{H}$ NMR $\left(500 \mathrm{MHz}, \mathrm{CDCl}_{3}\right) \delta 7.29-7.26(\mathrm{~m}, 2 \mathrm{H}), 7.19-7.17(\mathrm{~m}, 3 \mathrm{H}), 5.74(\mathrm{ddd}, J=$ $17.2,10.2,9.0 \mathrm{~Hz}, 1 \mathrm{H}), 5.26(\mathrm{dd}, J=10.3,1.8 \mathrm{~Hz}, 1 \mathrm{H}), 5.13(\mathrm{dd}, J=17.2,1.6 \mathrm{~Hz}, 1 \mathrm{H})$, $3.74-3.67(\mathrm{~m}, 4 \mathrm{H}), 2.75(\mathrm{dd}, J=13.9,8.8 \mathrm{~Hz}, 1 \mathrm{H}), 2.68(\mathrm{dd}, J=19.4,10.3 \mathrm{~Hz}, 1 \mathrm{H})$, $2.60-2.54(\mathrm{~m}, 3 \mathrm{H}), 2.45(\mathrm{dd}, J=17.0,3.5 \mathrm{~Hz}, 2 \mathrm{H}), 2.01-1.94(\mathrm{~m}, 1 \mathrm{H}), 1.76-1.68(\mathrm{~m}, 1 \mathrm{H})$.

${ }^{13} \mathbf{C ~ N M R}\left(126 \mathrm{MHz}, \mathrm{CDCl}_{3}\right) \delta 142.5,137.3,128.6,128.5,126.0,118.5,68.3,67.5,50.3,33.4,32.6$.

HPLC (Shimadzu LC-2030) (Daicel Chiralpak OD-H Column, ${ }^{i} \operatorname{PrOH}: n-H e x a n e=5: 95,1.0 \mathrm{ml} / \mathrm{min}$ ), R $t=$ $4.99 \mathrm{~min}$ (minor) and $5.42 \mathrm{~min}$ (major), >99\% ee.

$[\alpha]_{D}^{25}=-5.4\left(c 1.0, \mathrm{CHCl}_{3}\right)$.

HRMS (ESI): calculated $m / z$ for $\mathrm{C}_{15} \mathrm{H}_{21} \mathrm{NO}[\mathrm{M}+\mathrm{H}]^{+}=232.1696$, found 232.1700. 
(R)-ethyl 4-(5-phenylpent-1-en-3-yl)piperazine-1-carboxylate (7kb):

Clear oil, Yield: $43.8 \mathrm{mg}, 58 \%$.

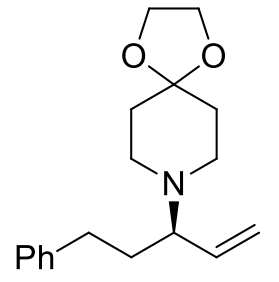

TLC $\mathbf{R}_{f}=0.2$ (PE:EA, 1:1).

${ }^{1} \mathbf{H}$ NMR $\left(400 \mathrm{MHz}, \mathrm{CDCl}_{3}\right) \delta 7.29-7.26(\mathrm{~m}, 2 \mathrm{H}), 7.18(\mathrm{dd}, J=7.2,3.7 \mathrm{~Hz}, 3 \mathrm{H}), 5.71$ (ddd, $J=17.2,10.2,9.0 \mathrm{~Hz}, 1 \mathrm{H}), 5.17(\mathrm{ddd}, J=18.7,13.7,1.7 \mathrm{~Hz}, 2 \mathrm{H}), 4.12(\mathrm{q}, J=7.1$ $\mathrm{Hz}, 2 \mathrm{H}), 3.45(\mathrm{dd}, J=6.3,3.7 \mathrm{~Hz}, 4 \mathrm{H}), 2.82(\mathrm{dd}, J=14.1,8.7 \mathrm{~Hz}, 1 \mathrm{H}), 2.71-2.54(\mathrm{~m}$, $4 \mathrm{H}), 2.40-2.37(\mathrm{~m}, 2 \mathrm{H}), 2.00-1.92(\mathrm{~m}, 1 \mathrm{H}), 1.78-1.68(\mathrm{~m}, 1 \mathrm{H}), 1.25(\mathrm{t}, J=7.1 \mathrm{~Hz}, 3 \mathrm{H})$.

${ }^{13}$ C NMR $\left(126 \mathrm{MHz}, \mathrm{CDCl}_{3}\right) \delta 155.6,142.4,136.9,128.6,128.5,126.0,118.5,67.8,61.4,49.3,44.2,33.7$, 32.6, 14.9.

HPLC (Shimadzu LC-16) (Daicel Chiralpak OD-H Column, ${ }^{i} \mathrm{PrOH}: n$-Hexane $=10: 90,0.5 \mathrm{~m} / \mathrm{min}$ ), R $t=$ $10.50 \mathrm{~min}$ (minor) and $11.87 \mathrm{~min}$ (major), $99 \%$ ee.

$[\alpha]_{D}^{25}=-15.1\left(c 1.0, \mathrm{CHCl}_{3}\right)$.

HRMS (ESI): calculated $m / z$ for $\mathrm{C}_{18} \mathrm{H}_{26} \mathrm{~N}_{2} \mathrm{O}_{2}[\mathrm{M}+\mathrm{H}]^{+}=303.2067$, found 303.2075.

(R)-8-(5-phenylpent-1-en-3-yl)-1,4-dioxa-8-azaspiro[4.5]decane (7kc):

Clear oil, Yield: $48.1 \mathrm{mg}, 67 \%$.

TLC $\mathbf{R}_{f}=0.3$ (PE:EA, 3:2).

${ }^{1}$ H NMR $\left(400 \mathrm{MHz}, \mathrm{CDCl}_{3}\right) \delta 7.29-7.25(\mathrm{~m}, 2 \mathrm{H}), 7.19-7.15(\mathrm{~m}, 3 \mathrm{H}), 5.81-5.72(\mathrm{~m}, 1 \mathrm{H}), 5.22(\mathrm{dd}, J=$ $10.3,1.9 \mathrm{~Hz}, 1 \mathrm{H}), 5.10$ (dd, $J=17.1,1.7 \mathrm{~Hz}, 1 \mathrm{H}), 3.94$ (s, 4H), 2.86 (dd, $J=14.0,8.8 \mathrm{~Hz}, 1 \mathrm{H}), 2.70-2.47$ (m, $6 \mathrm{H}), 1.97(\mathrm{dt}, J=15.6,5.8 \mathrm{~Hz}, 1 \mathrm{H}), 1.78-1.69(\mathrm{~m}, 5 \mathrm{H})$.

${ }^{13}$ C NMR $\left(126 \mathrm{MHz}, \mathrm{CDCl}_{3}\right) \delta 142.6,137.2,128.6,128.5,125.9,118.0,107.6,67.4$,<smiles>C=C[C@H](CCc1ccccc1)N1CCN(C(=O)CC)CC1</smiles>
64.4, 47.3, 35.4, 34.1, 32.9.

HPLC (Shimadzu LC-2030) (Daicel Chiralpak OJ-H Column, ${ }^{i} \mathrm{PrOH}: n-\mathrm{Hexane}=$ 0.2:99.8, $0.7 \mathrm{ml} / \mathrm{min}$ ), $\mathrm{R} t=13.60 \mathrm{~min}$ (major) and $14.48 \mathrm{~min}$ (minor), $>99 \%$ ee.

$[\alpha]_{D}^{25}=-10.8\left(c 1.0, \mathrm{CHCl}_{3}\right)$. 
HRMS (ESI): calculated $m / z$ for $\mathrm{C}_{18} \mathrm{H}_{25} \mathrm{NO}_{2}[\mathrm{M}+\mathrm{H}]^{+}=288.1958$, found 288.1960.

(R)-2-(5-phenylpent-1-en-3-yl)-1,2,3,4-tetrahydroisoquinoline (7kd):

Pale yellow oil; Yield: $55.5 \mathrm{mg}, 80 \%$.

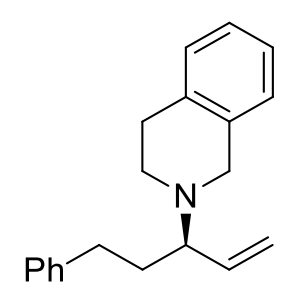

TLC $\mathbf{R}_{f}=0.2$ (PE:EA, 10:1).

${ }^{1} \mathbf{H}$ NMR $\left(500 \mathrm{MHz}, \mathrm{CDCl}_{3}\right) \delta 7.30(\mathrm{t}, J=7.5 \mathrm{~Hz}, 2 \mathrm{H}), 7.20(\mathrm{dd}, J=12.9,7.1 \mathrm{~Hz}, 3 \mathrm{H})$, $7.15-7.09(\mathrm{~m}, 3 \mathrm{H}), 7.03-7.02(\mathrm{~m}, 1 \mathrm{H}), 5.85(\mathrm{dd}, J=26.7,9.6 \mathrm{~Hz}, 1 \mathrm{H}), 5.30(\mathrm{dd}, J=$ $10.3,1.7 \mathrm{~Hz}, 1 \mathrm{H}), 5.20(\mathrm{~d}, J=18.6 \mathrm{~Hz}, 1 \mathrm{H}), 3.79(\mathrm{~d}, J=14.8 \mathrm{~Hz}, 1 \mathrm{H}), 3.69(\mathrm{~d}, J=$ $14.8 \mathrm{~Hz}, 1 \mathrm{H}), 3.03(\mathrm{t}, J=11.3 \mathrm{~Hz}, 1 \mathrm{H}), 2.97-2.92(\mathrm{~m}, 1 \mathrm{H}), 2.90(\mathrm{~d}, J=5.6 \mathrm{~Hz}, 2 \mathrm{H})$, $2.69(\mathrm{dd}, J=33.6,16.3 \mathrm{~Hz}, 3 \mathrm{H}), 2.14-2.07(\mathrm{~m}, 1 \mathrm{H}), 1.97-1.83(\mathrm{~m}, 1 \mathrm{H})$.

${ }^{13} \mathbf{C}$ NMR $\left(126 \mathrm{MHz}, \mathrm{CDCl}_{3}\right) \delta 142.6,137.1,135.5,134.8,128.9,128.7,128.5,126.9,126.1,125.9,125.7$, 118.4, 67.4, 52.7, 46.8, 34.0, 32.8, 29.8.

HPLC (Shimadzu LC-16) (Daicel Chiralpak IG Column, ${ }^{i} \operatorname{PrOH}: n$-Hexane $=0.2: 99.8,0.8 \mathrm{ml} / \mathrm{min}$ ), R $t=13.09$ $\min$ (minor) and $16.30 \mathrm{~min}$ (major), >99\% ee.

$[\alpha]_{D}^{25}=-18.5\left(c 0.5, \mathrm{CHCl}_{3}\right)$.

HRMS (ESI): calculated $m / z$ for $\mathrm{C}_{20} \mathrm{H}_{23} \mathrm{~N}[\mathrm{M}+\mathrm{H}]^{+}=278.1903$, found 278.1903.

\section{$(R)-N$-benzyl-5-phenylpent-1-en-3-amine (7ke): ${ }^{9}$}

Clear oil; Yield: $44.6 \mathrm{mg}, 71 \%$.

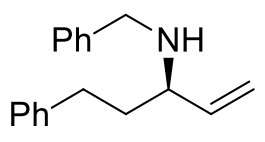

TLC R $\mathbf{R}_{f}: 0.4$ (EA:PE, 1:1).

${ }^{1} \mathbf{H}$ NMR $\left(400 \mathrm{MHz}, \mathrm{CDCl}_{3}\right) \delta 7.35-7.17(\mathrm{~m}, 10 \mathrm{H}), 5.75-5.68(\mathrm{~m}, 1 \mathrm{H}), 5.19(\mathrm{dd}, J=$ $22.4,13.7 \mathrm{~Hz}, 2 \mathrm{H}), 3.84$ (d, $J=13.1 \mathrm{~Hz}, 1 \mathrm{H}), 3.66$ (d, $J=13.1 \mathrm{~Hz}, 1 \mathrm{H}), 3.09$ (dd, $J=$ 13.7, $7.7 \mathrm{~Hz}, 1 \mathrm{H}), 2.65(\mathrm{dt}, J=13.9,7.8 \mathrm{~Hz}, 2 \mathrm{H}), 1.90-1.73(\mathrm{~m}, 2 \mathrm{H}), 1.47(\mathrm{~s}, 1 \mathrm{H})$.

${ }^{13}$ C NMR $\left(101 \mathrm{MHz}, \mathrm{CDCl}_{3}\right) \delta 142.4,141.2,140.9,128.6,128.5$ (for 2C), 128.4, 127.0, 125.9, 116.6, 60.9, $51.4,37.5,32.4$.

HPLC (Shimadzu LC-16) (Daicel Chiralpak OD-H Column, ${ }^{i} \mathrm{PrOH}: n-$ Hexane $=1.0: 99.0,1.0 \mathrm{ml} / \mathrm{min}$ ), R $t=$ $7.43 \mathrm{~min}$ (minor) and $8.12 \mathrm{~min}$ (major), >99\% ee.

$[\alpha]_{D}^{25}=+2.0\left(c 1.0, \mathrm{CHCl}_{3}\right)$. 
HRMS (ESI): calculated $m / z$ for $\mathrm{C}_{18} \mathrm{H}_{21} \mathrm{~N}[\mathrm{M}+\mathrm{H}]^{+}=252.1747$, found 252.1754.

(R)- $N$-phenethyl-5-phenylpent-1-en-3-amine (7kf):

White solid; Yield: $39.8 \mathrm{mg}, 60 \%$; M.P. $134{ }^{\circ} \mathrm{C}$.

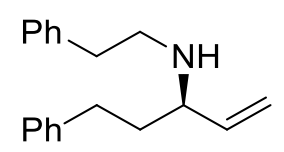

TLC $\mathbf{R}_{f}=0.2$ (PE:EA, 1:1).

${ }^{1} \mathbf{H}$ NMR $\left(500 \mathrm{MHz}, \mathrm{CDCl}_{3}\right) \delta 7.32-7.11(\mathrm{~m}, 10 \mathrm{H}), 5.70-5.62(\mathrm{~m}, 1 \mathrm{H}), 5.27(\mathrm{~d}, J=$ $10.2 \mathrm{~Hz}, 1 \mathrm{H}), 5.18(\mathrm{~d}, J=17.1 \mathrm{~Hz}, 1 \mathrm{H}), 3.59(\mathrm{~s}, 1 \mathrm{H}), 3.14(\mathrm{dd}, J=13.5,8.6 \mathrm{~Hz}, 1 \mathrm{H})$,

$2.99(\mathrm{dd}, J=12.9,8.6 \mathrm{~Hz}, 1 \mathrm{H}), 2.89-2.79(\mathrm{~m}, 3 \mathrm{H}), 2.63(\mathrm{dd}, J=19.2,10.0 \mathrm{~Hz}, 1 \mathrm{H}), 2.54(\mathrm{dd}, J=22.1,8.2$ $\mathrm{Hz}, 1 \mathrm{H}), 1.93(\mathrm{dd}, J=20.8,13.8 \mathrm{~Hz}, 1 \mathrm{H}), 1.80(\mathrm{dd}, J=20.1,11.4 \mathrm{~Hz}, 1 \mathrm{H})$.

${ }^{13}$ C NMR $\left(126 \mathrm{MHz}, \mathrm{CDCl}_{3}\right) \delta 142.2,141.1,140.2,128.9,128.9,128.6,128.5$ (for 2C), 126.3, 125.9, 116.6, $61.5,48.7,37.3,36.6,32.3$.

HPLC (Shimadzu LC-16) (Daicel Chiralpak OD-H Column, ${ }^{i} \mathrm{PrOH}: n-$ Hexane $=1.0: 99.0,0.7 \mathrm{ml} / \mathrm{min}$ ), R $t=$ $16.02 \mathrm{~min}$ (major) and $17.77 \mathrm{~min}$ (minor), $99 \%$ ee.

$[\alpha]_{D}^{25}=+3.7\left(c 1.0, \mathrm{CHCl}_{3}\right)$

HRMS (ESI): calculated $m / z$ for $\mathrm{C}_{19} \mathrm{H}_{23} \mathrm{~N}[\mathrm{M}+\mathrm{H}]^{+}=266.1903$, found 266.1904.

\section{(R)-5-phenyl- $N$-(prop-2-ynyl)pent-1-en-3-amine (7kg):}

Pale yellow oil; Yield: $33.4 \mathrm{mg}, 67 \%$.

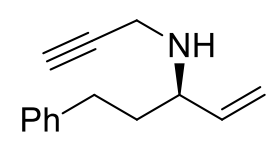

TLC $\mathbf{R}_{f}=0.5$ (PE:EA, 1:1).

${ }^{1} \mathbf{H}$ NMR $\left(500 \mathrm{MHz}, \mathrm{CDCl}_{3}\right) \delta 7.29-7.26(\mathrm{~m}, 2 \mathrm{H}), 7.18(\mathrm{dd}, J=7.4,5.2 \mathrm{~Hz}, 3 \mathrm{H}), 5.62$ $-5.55(\mathrm{~m}, 1 \mathrm{H}), 5.23-5.19(\mathrm{~m}, 2 \mathrm{H}), 3.45(\mathrm{dd}, J=17.0,2.5 \mathrm{~Hz}, 1 \mathrm{H}), 3.31(\mathrm{dd}, J=17.0$,

$2.4 \mathrm{~Hz}, 1 \mathrm{H}), 3.29-3.24(\mathrm{~m}, 1 \mathrm{H}), 2.71-2.58(\mathrm{~m}, 2 \mathrm{H}), 2.19(\mathrm{t}, J=2.4 \mathrm{~Hz}, 1 \mathrm{H}), 1.85-1.71(\mathrm{~m}, 2 \mathrm{H}), 1.35(\mathrm{~s}$, $1 \mathrm{H})$.

${ }^{13}$ C NMR $\left(101 \mathrm{MHz}, \mathrm{CDCl}_{3}\right) \delta 142.1,139.9,128.5,126.0,117.8,82.4,71.4,60.2,37.3,35.7,32.3$.

HPLC (Shimadzu LC-2030) (Daic el Chiralpak OD-H Column, ${ }^{i} \mathrm{PrOH}: n-\mathrm{Hexane}=0.8: 99.2,0.8 \mathrm{ml} / \mathrm{min}$ ), R $t=$ $9.71 \mathrm{~min}$ (minor) and $10.36 \mathrm{~min}$ (major), >99\% ee.

$[\alpha]_{D}^{25}=+27.8\left(c 1.0, \mathrm{CHCl}_{3}\right)$. 
HRMS (ESI): calculated $m / z$ for $\mathrm{C}_{14} \mathrm{H}_{17} \mathrm{~N}[\mathrm{M}+\mathrm{H}]^{+}=200.1434$, found 200.1427.

\section{$(R)-5$-phenyl- $N-((S)-1-p h e n y l e t h y l) p e n t-1-e n-3-a m i n e ~(7 k h):$}

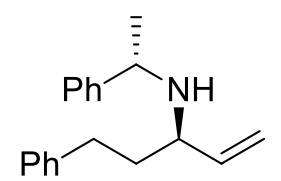

Pale yellow oil; Yield: $49.1 \mathrm{mg}, 74 \%$.

TLC $\mathbf{R}_{f}=0.2$ (PE:EA, 1:1).

${ }^{1} \mathbf{H}$ NMR $\left(500 \mathrm{MHz}, \mathrm{CDCl}_{3}\right) \delta 7.33-7.20(\mathrm{~m}, 7 \mathrm{H}), 7.14(\mathrm{t}, J=7.4 \mathrm{~Hz}, 1 \mathrm{H}), 7.08(\mathrm{~d}, J=$ $7.1 \mathrm{~Hz}, 2 \mathrm{H}), 5.62-5.55(\mathrm{~m}, 1 \mathrm{H}), 5.14-5.12(\mathrm{~m}, 1 \mathrm{H}), 4.95-4.91(\mathrm{~m}, 1 \mathrm{H}), 3.84(\mathrm{q}, J=6.7 \mathrm{~Hz}, 1 \mathrm{H}), 2.78(\mathrm{dd}$, $J=14.9,6.9 \mathrm{~Hz}, 1 \mathrm{H}), 2.63-2.49(\mathrm{~m}, 2 \mathrm{H}), 1.68(\mathrm{dd}, J=23.3,12.9 \mathrm{~Hz}, 2 \mathrm{H}), 1.37(\mathrm{~s}, 1 \mathrm{H}), 1.32(\mathrm{~d}, J=6.7 \mathrm{~Hz}$, $3 \mathrm{H})$.

${ }^{13} \mathbf{C}$ NMR $\left(126 \mathrm{MHz}, \mathrm{CDCl}_{3}\right) \delta 146.0,142.5,141.2,128.6,128.5,128.4,126.9,125.8,116.3,58.8,54.938 .2$, 32.5, 25.3.

$[\alpha]_{D}^{25}=-65.5\left(c 0.5, \mathrm{CHCl}_{3}\right)$.

HRMS (ESI): calculated $m / z$ for $\mathrm{C}_{19} \mathrm{H}_{23} \mathrm{~N}[\mathrm{M}+\mathrm{H}]^{+}=266.1903$, found 266.1908.

\section{(R)-5-phenyl- $N$-((R)-1-phenylethyl)pent-1-en-3-amine (7ki):}

Pale yellow oil; Yield: $50.4 \mathrm{mg}, 76 \%$.

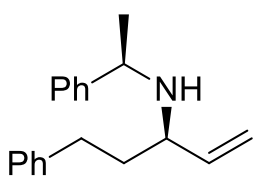

TLC $\mathbf{R}_{f}=0.2$ (PE:EA, 1:1).

${ }^{1} \mathbf{H}$ NMR $\left(500 \mathrm{MHz}, \mathrm{CDCl}_{3}\right) \delta 7.32-7.27(\mathrm{~m}, 5 \mathrm{H}), 7.26-7.16(\mathrm{~m}, 5 \mathrm{H}), 5.66-5.59$ (m, 1H), 5.09 (dd, $J=21.8,4.8 \mathrm{~Hz}, 2 \mathrm{H}), 3.86(\mathrm{q}, J=6.5 \mathrm{~Hz}, 1 \mathrm{H}), 3.09$ (dd, $J=13.1$, $7.9 \mathrm{~Hz}, 1 \mathrm{H}), 2.68(\mathrm{dd}, J=19.6,10.2 \mathrm{~Hz}, 1 \mathrm{H}), 2.61-2.55(\mathrm{~m}, 1 \mathrm{H}), 1.89-1.82(\mathrm{~m}, 1 \mathrm{H}), 1.74-1.67(\mathrm{~m}, 1 \mathrm{H})$, $1.47(\mathrm{~s}, 2 \mathrm{H}), 1.30(\mathrm{~d}, J=6.5 \mathrm{~Hz}, 3 \mathrm{H})$.

${ }^{13} \mathbf{C}$ NMR $\left(126 \mathrm{MHz}, \mathrm{CDCl}_{3}\right) \delta 146.4,142.5,141.4,128.6,128.5,127.0,126.8,125.9,115.9,58.5,54.7,36.9$, 32.3, 23.3.

$[\alpha]_{D}^{25}=+10.4\left(c 0.5, \mathrm{CHCl}_{3}\right)$

HRMS (ESI): calculated $m / z$ for $\mathrm{C}_{19} \mathrm{H}_{23} \mathrm{~N}[\mathrm{M}+\mathrm{H}]^{+}=266.1903$, found 266.1906. 


\section{(R)-5-phenyl- $N$-(2,2,2-trifluoroethyl)pent-1-en-3-amine (7kj):}

Clear oil, Yield: $51.1 \mathrm{mg}, 84 \%$.

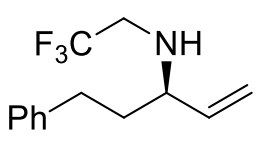

TLC $\mathbf{R}_{f}=0.7$ (PE:EA, 20:1).

${ }^{1} \mathbf{H}$ NMR $\left(400 \mathrm{MHz}, \mathrm{CDCl}_{3}\right) \delta 7.29(\mathrm{dd}, J=14.4,7.3 \mathrm{~Hz}, 2 \mathrm{H}), 7.20(\mathrm{t}, J=7.2 \mathrm{~Hz}, 3 \mathrm{H})$, $5.63-5.54(\mathrm{~m}, 1 \mathrm{H}), 5.24-5.15(\mathrm{~m}, 2 \mathrm{H}), 3.24-3.02(\mathrm{~m}, 3 \mathrm{H}), 2.67(\mathrm{tt}, J=21.0,10.3$

$\mathrm{Hz}, 2 \mathrm{H}), 1.87-1.71(\mathrm{~m}, 2 \mathrm{H}), 1.26(\mathrm{~s}, 1 \mathrm{H})$.

${ }^{13} \mathbf{C}$ NMR (126 MHz, $\left.\mathrm{CDCl}_{3}\right) \delta 142.0,140.0,128.6,129.46-122.18(\mathrm{~m}), 126.1,117.7,61.0,48.0(\mathrm{q}, J=31.2$ $\mathrm{Hz}), 37.4,32.3$.

HPLC (Shimadzu LC-2030) (Daicel Chiralpak IG Column, ${ }^{i} \operatorname{PrOH}: n-\mathrm{Hex}$ ane $=0.1: 99.9,1.0 \mathrm{ml} / \mathrm{min}$ ), R $t=$ $4.76 \mathrm{~min}$ (major) and $5.30 \mathrm{~min}$ (minor), >99\% ee.

$[\alpha]_{D}^{25}=+9.6\left(c 1.0, \mathrm{CHCl}_{3}\right)$.

HRMS (ESI): calculated $m / z$ for $\mathrm{C}_{13} \mathrm{H}_{16} \mathrm{~F}_{3} \mathrm{~N}[\mathrm{M}+\mathrm{H}]^{+}=244.1308$, found 244.1309.

\section{Gram scale synthes is of 3ab:}

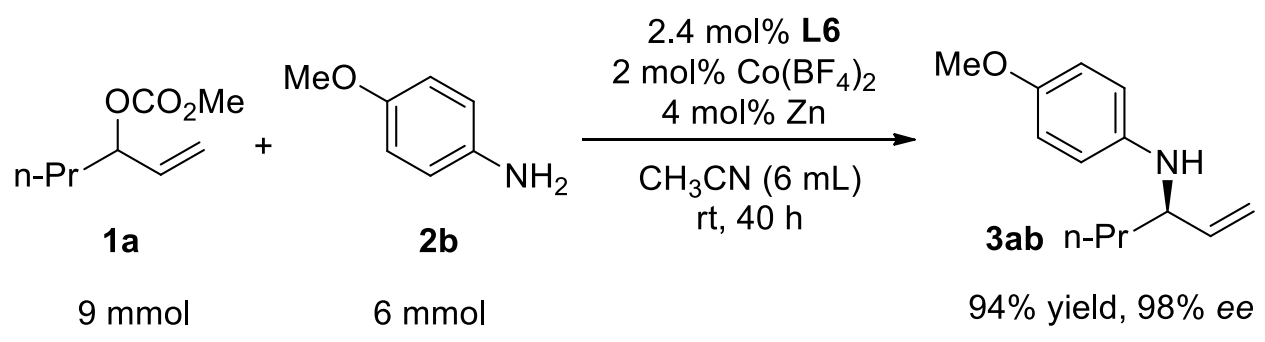

In an $\mathrm{N}_{2}$ filled glove box, a reaction tube was charged with $2.4 \mathrm{~mol} \%$ of $\mathbf{L 6}$ ( $80 \mathrm{mg}, 0.14 \mathrm{mmol}$ ), and $2 \mathrm{~mol} \%$ of $\mathrm{Co}\left(\mathrm{BF}_{4}\right)_{2}(28 \mathrm{mg}, 0.12 \mathrm{mmol})$ in $6 \mathrm{~mL}$ of acetonitrile. $\mathrm{Zn}$ dust (16 mg, $0.24 \mathrm{mmol}, 4 \mathrm{~mol} \%$ ) was added and the reaction mixture was stirred for 20 minutes. Then, $\mathbf{1 a}(1.420 \mathrm{~g}, 9 \mathrm{mmol})$ and $\mathbf{2 b}(0.740 \mathrm{~g}, 6 \mathrm{mmol})$ were added to the reaction mixture. The reaction was monitored by TLC and stopped after 40 hours. Solvent was removed and the residue was purified by flash column chromatography. Yield: $1.159 \mathrm{~g}$, 94\%; ee 98\%. 


\section{Procedure for the complex preparation and single crystal X-ray diffraction data:}

\section{$\left[\mathrm{L8}-\mathrm{Co}\left(\mathrm{CH}_{3} \mathrm{CN}\right)_{2}\right]\left(\mathrm{BF}_{4}\right)_{2}$ complex:}

To a stirred solution of $\mathbf{L 8}(0.265 \mathrm{~g}, 0.5 \mathrm{mmol})$ in acetonitrile $(2 \mathrm{~mL})$ was added 1 eq of anhydrous $\mathrm{Co}\left(\mathrm{BF}_{4}\right)_{2}$ $(0.117 \mathrm{~g}, 0.5 \mathrm{mmol})$ and the resulting mixture was stirred for 4 hours at room temperature under an $\mathrm{N}_{2}$ atmosphere. The reaction mixture was concentrated and washed with diethyl ether several times to obtained a brown solid. A single crystal suitable for X-ray diffraction analysis was obtained from acetonitrile/diethyl ether solvent. Yield: $0.354 \mathrm{~g}, 84 \%$.

\section{$\left[\mathrm{L9}-\mathrm{Co}\left(\mathrm{CH}_{3} \mathrm{CN}\right)_{2}\right]\left(\mathrm{BF}_{4}\right)_{2}$ complex:}

[L9-Co( $\left.\left(\mathrm{CH}_{3} \mathrm{CN}\right)_{2}\right]\left(\mathrm{BF}_{4}\right)_{2}$ was synthesized in a similar way to that of $\left[\mathrm{L8}-\mathrm{Co}\left(\mathrm{CH}_{3} \mathbf{C N}\right)_{2}\right]\left(\mathrm{BF}_{4}\right)_{2}$. Single crystal suitable for X-ray study was grown from acetonitrile/diethyl ether solvent. Yield: $0.352 \mathrm{~g}, 85 \%$.

\section{L4CoI•THF complex:}

An ethanol solution of $\mathbf{L 4}(0.216 \mathrm{~g}, 0.5 \mathrm{mmol})$ in $8 \mathrm{~mL}$ was added 1 eq of $\mathrm{CoI}_{2}(0.157 \mathrm{~g}, 0.5 \mathrm{mmol})$ and the mixture was stirred for 30 minutes under an $\mathrm{N}_{2}$ atmosphere. A brown precipitate appeared in the reaction mixture. $\mathrm{NaBH}_{4}(0.019 \mathrm{~g}, 0.5 \mathrm{mmol}, 1 \mathrm{eq})$ was added portion wise to the reaction mixture. During this period, the brown solid turns green. After 4 hours, the green precipitate was isolated by filtration and washed one time with ethanol under $\mathrm{N}_{2}$ atmosphere. Yield: $0.280 \mathrm{~g}, 82 \%$. Single crystal suitable for X-ray study was grown from $\mathrm{THF} / \mathrm{Hexane.}$

X-ray crystallographic data were collected using a Bruker APEX-II CCD diffractometer, equipped with a sealed tube $\mathrm{Cu}-\mathrm{K} \alpha$ radiation ( $\lambda=1.54178 \AA$ ) at ambient temperature or at low temperature under liquid $\mathrm{N}_{2}$ flow. Structure was solved with the Superflip, structure solution program using Charge Flipping and refined by direct methods using SHELXL-2016/6 and with full-matrix least squares on $F^{2}$ using SHELXL-2016/6. All the non-hydrogen atoms were refined anisotropically. 


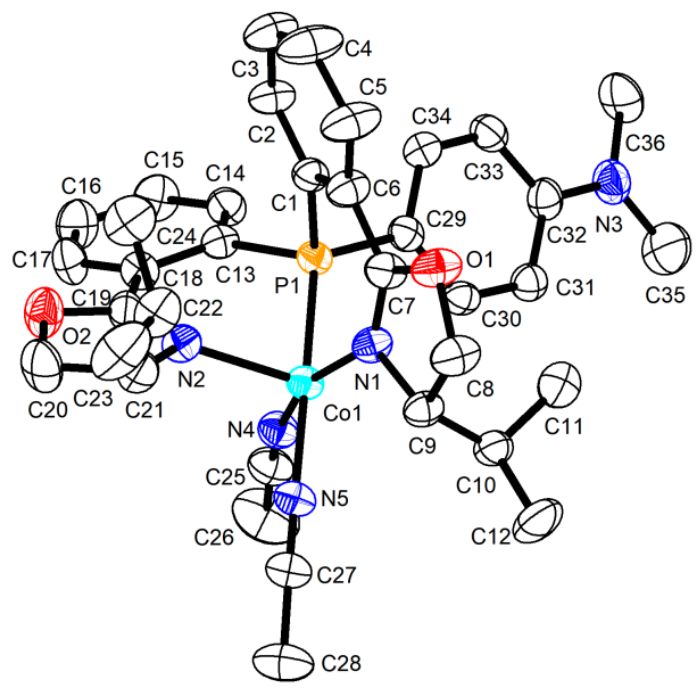

Figure 1: Dicationic complex structure with atom labeling scheme of [ $\left.\mathbf{L 8}-\mathbf{C o}\left(\mathbf{C H}_{3} \mathbf{C N}\right)_{2}\right]\left(\mathbf{B F}_{4}\right)_{2} . \mathrm{H}$ atoms and counter anions are omitted for structural clarity. ORTEP diagram are drawn at the $30 \%$ probability level.

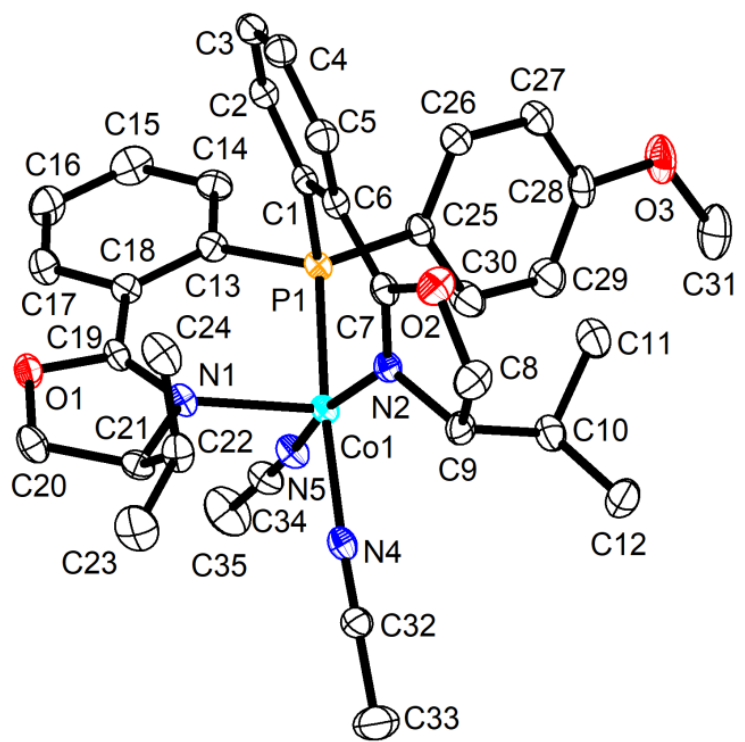

Figure 2: Dicationic complex structure with atom labeling scheme of $\left[\mathbf{L 9}-\mathbf{C o}\left(\mathbf{C H}_{3} \mathbf{C N}\right)_{2}\right]\left(\mathbf{B F}_{4}\right)_{2}$. H atoms and counter anions are omitted for structural clarity. ORTEP diagram are drawn at the $30 \%$ probability level. 


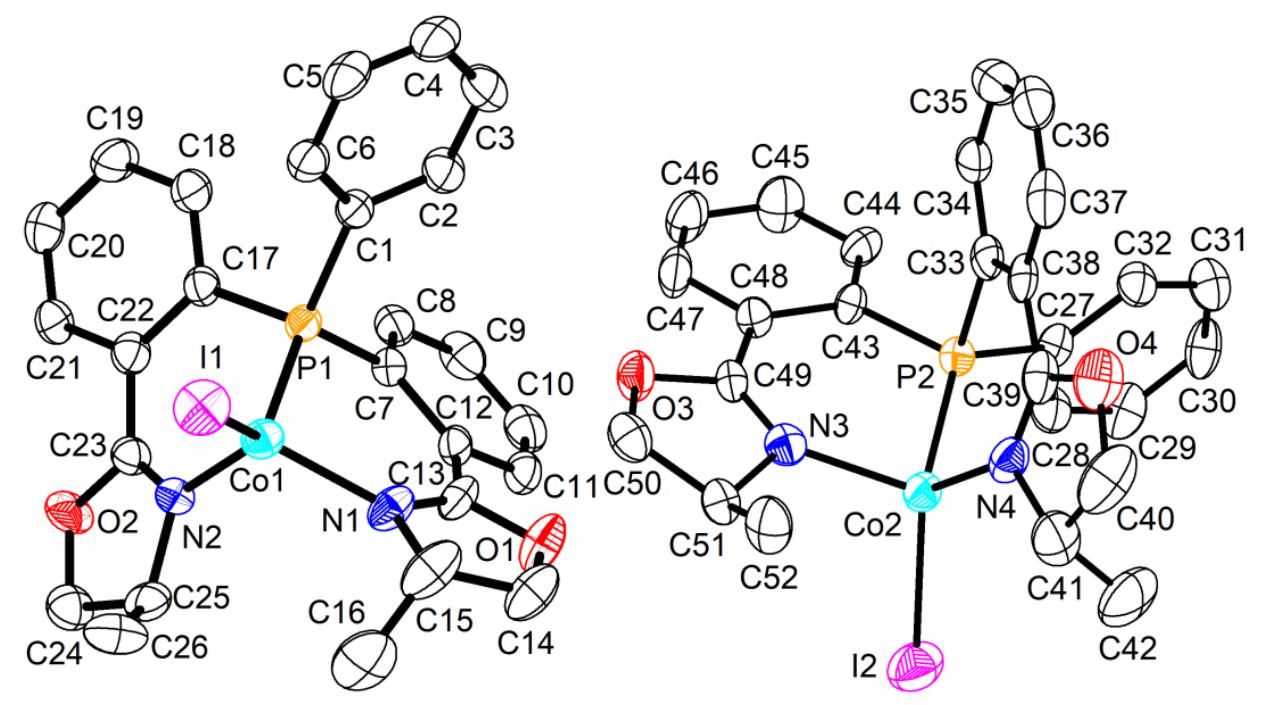

Figure 3: ORTEP diagram of the two distinct molecules of L4CoI•THF present in the unit cell are shown at the $30 \%$ probability level. $\mathrm{H}$ atoms and solvent molecules are omitted for the clarity.

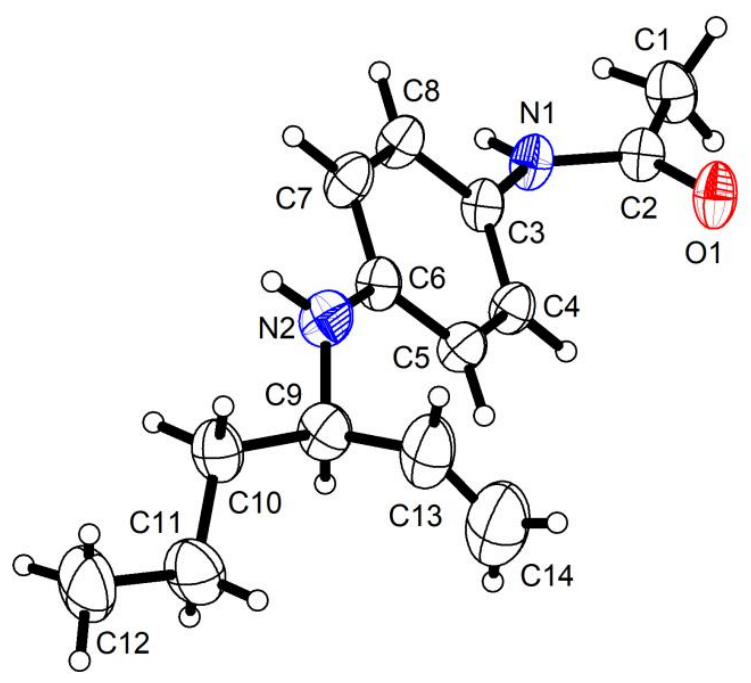

Figure 4: ORTEP diagram of 3ag with atom labeling scheme are shown at 30\% probability level.

Table 1: Selected bond distances $(\AA)$ for $\left[\mathbf{L 8}-\mathbf{C o}\left(\mathbf{C H}_{3} \mathbf{C N}\right)_{2}\right]\left(\mathbf{B F}_{4}\right)_{2}$ are listed below.

\begin{tabular}{llllll}
\hline Co1-N4 & $1.917(10)$ & N2-C19 & $1.268(13)$ & C14-C15 & $1.364(16)$ \\
Co1-N5 & $1.946(8)$ & N2-C21 & $1.478(14)$ & C17-C16 & $1.367(17)$ \\
Co1-N1 & $1.951(7)$ & N3-C32 & $1.366(15)$ & C17-C18 & $1.388(15)$ \\
Co1-N2 & $2.102(9)$ & N3-C35 & $1.419(18)$ & C16-C15 & $1.366(18)$ \\
Co1-P1 & $2.158(2)$ & N3-C36 & $1.443(15)$ & C18-C19 & $1.484(14)$ \\
C1-C2 & $1.380(14)$ & C3-C4 & $1.370(19)$ & C20-C21 & $1.555(19)$ \\
C1-C6 & $1.399(14)$ & C4-C5 & $1.398(19)$ & C21-C22 & $1.546(19)$ \\
C1-P1 & $1.824(10)$ & N4-C25 & $1.128(14)$ & C22-C24 & $1.52(2)$ \\
O1-C7 & $1.333(11)$ & N5-C27 & $1.132(12)$ & C22-C23 & $1.528(19)$ \\
O1-C8 & $1.463(13)$ & C5-C6 & $1.372(16)$ & C25-C26 & $1.453(19)$ \\
\hline
\end{tabular}




\begin{tabular}{llllll}
\hline $\mathrm{N} 1-\mathrm{C} 7$ & $1.282(12)$ & $\mathrm{C} 6-\mathrm{C} 7$ & $1.463(15)$ & $\mathrm{C} 27-\mathrm{C} 28$ & $1.455(16)$ \\
$\mathrm{N} 1-\mathrm{C} 9$ & $1.503(13)$ & $\mathrm{C} 8-\mathrm{C} 9$ & $1.518(15)$ & $\mathrm{C} 29-\mathrm{C} 30$ & $1.387(15)$ \\
$\mathrm{P} 1-\mathrm{C} 29$ & $1.802(10)$ & $\mathrm{C} 9-\mathrm{C} 10$ & $1.505(16)$ & $\mathrm{C} 29-\mathrm{C} 34$ & $1.404(14)$ \\
$\mathrm{P} 1-\mathrm{C} 13$ & $1.829(9)$ & $\mathrm{C} 10-\mathrm{C} 11$ & $1.522(18)$ & $\mathrm{C} 30-\mathrm{C} 31$ & $1.364(15)$ \\
$\mathrm{C} 2-\mathrm{C} 3$ & $1.383(17)$ & $\mathrm{C} 10-\mathrm{C} 12$ & $1.532(16)$ & $\mathrm{C} 31-\mathrm{C} 32$ & $1.418(15)$ \\
$\mathrm{O} 2-\mathrm{C} 19$ & $1.337(13)$ & $\mathrm{C} 13-\mathrm{C} 14$ & $1.389(14)$ & $\mathrm{C} 32-\mathrm{C} 33$ & $1.415(16)$ \\
$\mathrm{O} 2-\mathrm{C} 20$ & $1.444(15)$ & $\mathrm{C} 13-\mathrm{C} 18$ & $1.400(14)$ & $\mathrm{C} 33-\mathrm{C} 34$ & $1.366(16)$ \\
\hline
\end{tabular}

Table 2: Selected bond angles $\left(^{\circ}\right)$ for $\left[\mathbf{L 8}-\mathbf{C o}\left(\mathbf{C H}_{3} \mathbf{C N}\right)_{2}\right]\left(\mathbf{B F}_{4}\right)_{2}$ are listed below .

\begin{tabular}{llll}
\hline N4-Co1-N5 & $86.7(3)$ & C13-P1-Co1 & $107.9(3)$ \\
N4-Co1-N1 & $159.9(4)$ & C19-N2-Co1 & $126.5(7)$ \\
N5-Co1-N1 & $92.8(3)$ & C21-N2-Co1 & $124.4(7)$ \\
N4-Co1-N2 & $98.6(4)$ & C25-N4-Co1 & $170.2(9)$ \\
N5-Co1-N2 & $95.7(4)$ & C27-N5-Co1 & $167.7(9)$ \\
N1-Co1-N2 & $101.4(4)$ & C29-P1-C1 & $106.3(4)$ \\
N4-Co1-P1 & $89.4(3)$ & C29-P1-C13 & $106.8(4)$ \\
N5-Co1-P1 & $176.1(3)$ & C1-P1-C13 & $106.1(4)$ \\
N1-Co1-P1 & $90.9(2)$ & N1-C7-C6 & $128.7(8)$ \\
N2-Co1-P1 & $84.9(2)$ & C1-C6-C7 & $122.8(9)$ \\
C7-N1-Co1 & $131.8(6)$ & C6-C1-P1 & $120.4(7)$ \\
C9-N1-Co1 & $119.6(6)$ & C18-C13-P1 & $120.4(7)$ \\
C29-P1-Co1 & $118.8(3)$ & C13-C18-C19 & $123.0(9)$ \\
C1-P1-Co1 & $110.3(3)$ & N2-C19-C18 & $126.9(9)$ \\
\hline
\end{tabular}

Table 3: Selected bond distances $(\AA)$ for $\left[\mathbf{L 9}-\mathbf{C o}\left(\mathbf{C H}_{3} \mathbf{C N}\right)_{2}\right]\left(\mathbf{B F}_{4}\right)_{2}$ are listed below.

\begin{tabular}{llllll}
\hline Co1-N5 & $1.937(5)$ & $\mathrm{C} 3-\mathrm{C} 4$ & $1.390(9)$ & $\mathrm{C} 15-\mathrm{C} 16$ & $1.38(1)$ \\
Co1-N2 & $1.952(5)$ & $\mathrm{O} 2-\mathrm{C} 7$ & $1.341(7)$ & $\mathrm{C} 16-\mathrm{C} 17$ & $1.370(9)$ \\
Co1-N4 & $1.972(5)$ & $\mathrm{O} 2-\mathrm{C} 8$ & $1.453(8)$ & $\mathrm{C} 19-\mathrm{C} 18$ & $1.477(7)$ \\
Co1-N1 & $2.073(4)$ & $\mathrm{N} 2-\mathrm{C} 7$ & $1.288(7)$ & $\mathrm{C} 18-\mathrm{C} 17$ & $1.406(8)$ \\
Co1-P1 & $2.1523(15)$ & $\mathrm{N} 2-\mathrm{C} 9$ & $1.503(7)$ & $\mathrm{C} 20-\mathrm{C} 21$ & $1.526(8)$ \\
$\mathrm{C} 1-\mathrm{C} 2$ & $1.388(8)$ & $\mathrm{C} 5-\mathrm{C} 4$ & $1.377(9)$ & $\mathrm{C} 21-\mathrm{C} 22$ & $1.532(8)$ \\
$\mathrm{C} 1-\mathrm{C} 6$ & $1.419(7)$ & $\mathrm{C} 5-\mathrm{C} 6$ & $1.401(8)$ & $\mathrm{C} 22-\mathrm{C} 24$ & $1.517(10)$ \\
$\mathrm{C} 1-\mathrm{P} 1$ & $1.814(5)$ & $\mathrm{N} 5-\mathrm{C} 34$ & $1.123(8)$ & $\mathrm{C} 22-\mathrm{C} 23$ & $1.527(8)$ \\
$\mathrm{N} 1-\mathrm{C} 19$ & $1.279(7)$ & $\mathrm{N} 4-\mathrm{C} 32$ & $1.141(8)$ & $\mathrm{C} 25-\mathrm{C} 26$ & $1.379(8)$ \\
$\mathrm{N} 1-\mathrm{C} 21$ & $1.485(6)$ & $\mathrm{C} 7-\mathrm{C} 6$ & $1.446(8)$ & $\mathrm{C} 25-\mathrm{C} 30$ & $1.390(8)$ \\
$\mathrm{O} 1-\mathrm{C} 19$ & $1.345(6)$ & $\mathrm{C} 8-\mathrm{C} 9$ & $1.515(8)$ & $\mathrm{C} 26-\mathrm{C} 27$ & $1.383(9)$ \\
$\mathrm{O} 1-\mathrm{C} 20$ & $1.448(7)$ & $\mathrm{C} 9-\mathrm{C} 10$ & $1.549(8)$ & $\mathrm{C} 27-\mathrm{C} 28$ & $1.372(10)$ \\
$\mathrm{P} 1-\mathrm{C} 25$ & $1.810(5)$ & $\mathrm{C} 10-\mathrm{C} 11$ & $1.516(9)$ & $\mathrm{C} 28-\mathrm{C} 29$ & $1.385(9)$ \\
$\mathrm{P} 1-\mathrm{C} 13$ & $1.809(5)$ & $\mathrm{C} 10-\mathrm{C} 12$ & $1.534(9)$ & $\mathrm{C} 29-\mathrm{C} 30$ & $1.390(9)$ \\
$\mathrm{O} 3-\mathrm{C} 28$ & $1.372(7)$ & $\mathrm{C} 13-\mathrm{C} 14$ & $1.395(8)$ & $\mathrm{C} 32-\mathrm{C} 33$ & $1.452(9)$ \\
$\mathrm{O} 3-\mathrm{C} 31$ & $1.437(10)$ & $\mathrm{C} 13-\mathrm{C} 18$ & $1.410(8)$ & $\mathrm{C} 34-\mathrm{C} 35$ & $1.446(9)$ \\
$\mathrm{C} 3-\mathrm{C} 2$ & $1.376(8)$ & $\mathrm{C} 14-\mathrm{C} 15$ & $1.392(9)$ & & \\
& & & & & \\
\hline
\end{tabular}

Table 4: Selected bond angles $\left(^{\circ}\right)$ for $\left[\mathbf{L 9 - C o}\left(\mathbf{C H}_{3} \mathbf{C N}\right)_{2}\right]\left(\mathbf{B F}_{4}\right)_{2}$ are listed below.

\begin{tabular}{llll}
\hline N5-Co1-N2 & $166.3(2)$ & C34-N5-Co1 & $173.5(5)$ \\
N5-Co1-N4 & $86.5(2)$ & C32-N4-Co1 & $170.2(5)$ \\
N2-Co1-N4 & $92.81(19)$ & C25-P1-Co1 & $113.32(19)$ \\
N5-Co1-N1 & $90.02(19)$ & C13-P1-Co1 & $110.19(18)$ \\
N2-Co1-N1 & $103.66(18)$ & C1-P1-Co1 & $113.93(17)$ \\
N4-Co1-N1 & $97.66(18)$ & C25-P1-C13 & $109.9(2)$ \\
N5-Co1-P1 & $89.76(15)$ & C25-P1-C1 & $106.8(2)$ \\
N2-Co1-P1 & $89.38(14)$ & C13-P1-C1 & $102.1(2)$ \\
N4-Co1-P1 & $172.91(15)$ & N2-C7-C6 & $129.6(5)$ \\
N1-Co1-P1 & $88.37(13)$ & C1-C6-C7 & $122.4(5)$ \\
C19-N1-Co1 & $128.8(3)$ & C6-C1-P1 & $120.1(4)$ \\
C21-N1-Co1 & $122.0(3)$ & C18-C13-P1 & $120.5(4)$ \\
\hline
\end{tabular}




\begin{tabular}{llll}
\hline C7-N2-Co1 & $132.4(4)$ & $\mathrm{C} 13-\mathrm{C} 18-\mathrm{C} 19$ & $123.7(4)$ \\
$\mathrm{C} 9-\mathrm{N} 2-\mathrm{Co} 1$ & $120.5(3)$ & $\mathrm{N} 1-\mathrm{C} 19-\mathrm{C} 18$ & $128.2(5)$ \\
\hline
\end{tabular}

Table 5: Selected bond distances $(\AA)$ for $\mathbf{L 4 - C o I} \bullet \mathbf{T H F}$ are listed below.

\begin{tabular}{llllll}
\hline Co1-I1 & $2.5529(16)$ & $\mathrm{N} 2-\mathrm{C} 23$ & $1.272(13)$ & $\mathrm{C} 11-\mathrm{C} 12$ & $1.392(13)$ \\
Co1-N1 & $2.015(8)$ & $\mathrm{N} 2-\mathrm{C} 25$ & $1.496(12)$ & $\mathrm{C} 12-\mathrm{C} 13$ & $1.462(15)$ \\
Co1-N2 & $2.024(8)$ & $\mathrm{C} 1-\mathrm{C} 6$ & $1.400(13)$ & $\mathrm{C} 14-\mathrm{C} 15$ & $1.504(17)$ \\
Co1-P1 & $2.159(3)$ & $\mathrm{C} 1-\mathrm{C} 2$ & $1.400(13)$ & $\mathrm{C} 19-\mathrm{C} 18$ & $1.377(14)$ \\
P1-C7 & $1.820(9)$ & $\mathrm{C} 2-\mathrm{C} 3$ & $1.391(14)$ & $\mathrm{C} 19-\mathrm{C} 20$ & $1.406(15)$ \\
P1-C17 & $1.827(9)$ & $\mathrm{C} 3-\mathrm{C} 4$ & $1.402(16)$ & $\mathrm{C} 18-\mathrm{C} 17$ & $1.387(14)$ \\
P1-C1 & $1.820(9)$ & $\mathrm{C} 4-\mathrm{C} 5$ & $1.388(17)$ & $\mathrm{C} 17-\mathrm{C} 22$ & $1.409(12)$ \\
O1-C13 & $1.353(11)$ & $\mathrm{C} 9-\mathrm{C} 10$ & $1.369(15)$ & $\mathrm{C} 16-\mathrm{C} 15$ & $1.55(2)$ \\
O1-C14 & $1.448(15)$ & $\mathrm{C} 9-\mathrm{C} 8$ & $1.400(13)$ & $\mathrm{C} 20-\mathrm{C} 21$ & $1.358(15)$ \\
O2-C23 & $1.366(11)$ & $\mathrm{C} 8-\mathrm{C} 7$ & $1.380(14)$ & $\mathrm{C} 21-\mathrm{C} 22$ & $1.406(13)$ \\
O2-C24 & $1.455(14)$ & $\mathrm{C} 7-\mathrm{C} 12$ & $1.454(13)$ & $\mathrm{C} 22-\mathrm{C} 23$ & $1.479(13)$ \\
N1-C13 & $1.280(13)$ & $\mathrm{C} 6-\mathrm{C} 5$ & $1.389(15)$ & $\mathrm{C} 24-\mathrm{C} 25$ & $1.521(14)$ \\
N1-C15 & $1.512(13)$ & $\mathrm{C} 11-\mathrm{C} 10$ & $1.377(15)$ & $\mathrm{C} 25-\mathrm{C} 26$ & $1.498(17)$ \\
& & & & & \\
Co2-I2 & $2.5475(16)$ & $\mathrm{N} 4-\mathrm{C} 39$ & $1.281(13)$ & $\mathrm{C} 37-\mathrm{C} 38$ & $1.416(13)$ \\
Co2-N3 & $2.007(7)$ & $\mathrm{N} 4-\mathrm{C} 41$ & $1.492(13)$ & $\mathrm{C} 38-\mathrm{C} 39$ & $1.474(15)$ \\
Co2-N4 & $2.039(8)$ & $\mathrm{C} 27-\mathrm{C} 32$ & $1.403(13)$ & $\mathrm{C} 40-\mathrm{C} 41$ & $1.525(19)$ \\
Co2-P2 & $2.156(3)$ & $\mathrm{C} 27-\mathrm{C} 28$ & $1.409(13)$ & $\mathrm{C} 41-\mathrm{C} 42$ & $1.505(18)$ \\
P2-C27 & $1.822(9)$ & $\mathrm{C} 28-\mathrm{C} 29$ & $1.363(13)$ & $\mathrm{C} 43-\mathrm{C} 44$ & $1.393(13)$ \\
P2-C43 & $1.835(8)$ & $\mathrm{C} 29-\mathrm{C} 30$ & $1.386(15)$ & $\mathrm{C} 43-\mathrm{C} 48$ & $1.413(11)$ \\
P2-C33 & $1.864(9)$ & $\mathrm{C} 30-\mathrm{C} 31$ & $1.394(15)$ & $\mathrm{C} 44-\mathrm{C} 45$ & $1.408(14)$ \\
O3-C49 & $1.376(10)$ & $\mathrm{C} 31-\mathrm{C} 32$ & $1.377(13)$ & $\mathrm{C} 45-\mathrm{C} 46$ & $1.379(15)$ \\
O3-C50 & $1.462(12)$ & $\mathrm{C} 33-\mathrm{C} 34$ & $1.389(15)$ & $\mathrm{C} 46-\mathrm{C} 47$ & $1.380(15)$ \\
O4-C39 & $1.348(11)$ & $\mathrm{C} 33-\mathrm{C} 38$ & $1.390(13)$ & $\mathrm{C} 47-\mathrm{C} 48$ & $1.395(13)$ \\
O4-C40 & $1.419(16)$ & $\mathrm{C} 34-\mathrm{C} 35$ & $1.377(15)$ & $\mathrm{C} 48-\mathrm{C} 49$ & $1.476(12)$ \\
N3-C49 & $1.278(12)$ & $\mathrm{C} 35-\mathrm{C} 36$ & $1.403(16)$ & $\mathrm{C} 50-\mathrm{C} 51$ & $1.519(14)$ \\
N3-C51 & $1.498(12)$ & $\mathrm{C} 36-\mathrm{C} 37$ & $1.375(16)$ & $\mathrm{C} 51-\mathrm{C} 52$ & $1.490(15)$ \\
\hline
\end{tabular}

Table 6: Selected bond angles $\left({ }^{\circ}\right)$ for $\mathbf{L} 4-\mathbf{C o I} \bullet \mathbf{T H F}$ are listed below .

\begin{tabular}{llll}
\hline N1-Co1-N2 & $106.7(3)$ & N3-Co2-N4 & $108.9(3)$ \\
N1-Co1-P1 & $94.3(3)$ & N3-Co2-P2 & $91.0(2)$ \\
N2-Co1-P1 & $90.8(2)$ & N4-Co2-P2 & $93.3(2)$ \\
N1-Co1-I1 & $115.8(2)$ & N3-Co2-I2 & $109.3(2)$ \\
N2-Co1-I1 & $121.5(2)$ & N4-Co2-I2 & $120.4(2)$ \\
P1-Co1-I1 & $122.27(8)$ & P2-Co2-I2 & $129.42(9)$ \\
C7-P1-Co1 & $110.3(3)$ & C27-P2-Co2 & $123.0(3)$ \\
C1-P1-Co1 & $124.4(3)$ & C43-P2-Co2 & $112.2(3)$ \\
C17-P1-Co1 & $111.3(3)$ & C33-P2-Co2 & $111.0(3)$ \\
C7-P1-C1 & $103.6(4)$ & C27-P2-C43 & $103.7(4)$ \\
C7-P1-C17 & $101.5(4)$ & C27-P2-C33 & $103.2(4)$ \\
C1-P1-C17 & $103.3(4)$ & C43-P2-C33 & $101.4(4)$ \\
C23-N2-Co1 & $130.2(6)$ & C49-N3-Co2 & $131.6(6)$ \\
C25-N2-Co1 & $121.2(6)$ & C51-N3-Co2 & $119.7(6)$ \\
C13-N1-Co1 & $129.3(7)$ & C39-N4-Co2 & $130.2(7)$ \\
C15-N1-Co1 & $122.1(7)$ & C41-N4-Co2 & $122.1(7)$ \\
C22-C17-P1 & $120.9(7)$ & C48-C43-P2 & $121.5(6)$ \\
C12-C7-P1 & $121.7(7)$ & C38-C33-P2 & $122.3(7)$ \\
N1-C13-C12 & $129.3(8)$ & N3-C49-C48 & $129.9(8)$ \\
C7-C12-C13 & $123.5(9)$ & C43-C48-C49 & $122.9(8)$ \\
C23-N2-C25 & $108.6(8)$ & N4-C41-C42 & $110.8(10)$ \\
N2-C25-C26 & $112.1(8)$ & N4-C41-C40 & $102.5(10)$ \\
N2-C25-C24 & $102.5(8)$ & C49-N3-C51 & $107.5(7)$ \\
C17-C22-C23 & $123.6(8)$ & N4-C39-C38 & $128.9(8)$ \\
N2-C23-C22 & $129.2(9)$ & C33-C38-C39 & $125.3(8)$ \\
\hline
\end{tabular}


Table 7: Selected bond distances $(\AA)$ and bond angles $\left({ }^{\circ}\right)$ for compound 3ag are listed below.

\begin{tabular}{llll}
\hline $\mathrm{O} 1-\mathrm{C} 2$ & $1.235(3)$ & $\mathrm{O} 1-\mathrm{C} 2-\mathrm{N} 1$ & $122.6(3)$ \\
$\mathrm{C} 1-\mathrm{C} 2$ & $1.503(4)$ & $\mathrm{O} 1-\mathrm{C} 2-\mathrm{C} 1$ & $121.6(3)$ \\
$\mathrm{N} 1-\mathrm{C} 2$ & $1.336(4)$ & $\mathrm{N} 1-\mathrm{C} 2-\mathrm{C} 1$ & $115.8(3)$ \\
$\mathrm{N} 1-\mathrm{C} 3$ & $1.427(4)$ & $\mathrm{C} 4-\mathrm{C} 3-\mathrm{C} 8$ & $118.5(3)$ \\
$\mathrm{N} 2-\mathrm{C} 6$ & $1.386(4)$ & $\mathrm{C} 4-\mathrm{C} 3-\mathrm{N} 1$ & $122.7(3)$ \\
$\mathrm{N} 2-\mathrm{C} 9$ & $1.425(5)$ & $\mathrm{C} 8-\mathrm{C} 3-\mathrm{N} 1$ & $118.8(3)$ \\
$\mathrm{C} 3-\mathrm{C} 4$ & $1.379(4)$ & $\mathrm{N} 2-\mathrm{C} 6-\mathrm{C} 7$ & $119.1(3)$ \\
$\mathrm{C} 3-\mathrm{C} 8$ & $1.386(4)$ & $\mathrm{N} 2-\mathrm{C} 6-\mathrm{C} 5$ & $123.2(3)$ \\
$\mathrm{C} 6-\mathrm{C} 7$ & $1.396(5)$ & $\mathrm{C} 7-\mathrm{C} 6-\mathrm{C} 5$ & $117.8(3)$ \\
$\mathrm{C} 6-\mathrm{C} 5$ & $1.400(4)$ & $\mathrm{C} 4-\mathrm{C} 5-\mathrm{C} 6$ & $120.7(3)$ \\
$\mathrm{C} 5-\mathrm{C} 4$ & $1.383(4)$ & $\mathrm{C} 3-\mathrm{C} 4-\mathrm{C} 5$ & $120.9(3)$ \\
$\mathrm{C} 7-\mathrm{C} 8$ & $1.375(5)$ & $\mathrm{C} 8-\mathrm{C} 7-\mathrm{C} 6$ & $120.8(3)$ \\
$\mathrm{C} 9-\mathrm{C} 13$ & $1.509(6)$ & $\mathrm{N} 2-\mathrm{C} 9-\mathrm{C} 13$ & $108.9(4)$ \\
$\mathrm{C} 9-\mathrm{C} 10$ & $1.534(5)$ & $\mathrm{N} 2-\mathrm{C} 9-\mathrm{C} 10$ & $109.4(3)$ \\
$\mathrm{C} 10-\mathrm{C} 11$ & $1.504(7)$ & $\mathrm{C} 13-\mathrm{C} 9-\mathrm{C} 10$ & $110.0(3)$ \\
$\mathrm{C} 11-\mathrm{C} 12$ & $1.512(7)$ & $\mathrm{C} 7-\mathrm{C} 8-\mathrm{C} 3$ & $121.3(3)$ \\
$\mathrm{C} 13-\mathrm{C} 14$ & $1.194(8)$ & $\mathrm{C} 11-\mathrm{C} 10-\mathrm{C} 9$ & $115.0(4)$ \\
$\mathrm{C} 2-\mathrm{N} 1-\mathrm{C} 3$ & $126.5(2)$ & $\mathrm{C} 10-\mathrm{C} 11-\mathrm{C} 12$ & $111.9(5)$ \\
$\mathrm{C} 6-\mathrm{N} 2-\mathrm{C} 9$ & $124.7(3)$ & $\mathrm{C} 14-\mathrm{C} 13-\mathrm{C} 9$ & $131.5(7)$ \\
\hline
\end{tabular}

Table 8: Crystallographic data and structure refinement for $\left[\mathbf{L 8}-\mathbf{C o}\left(\mathrm{CH}_{3} \mathbf{C N}\right)_{2}\right]\left(\mathbf{B F}_{4}\right)_{2} ;$; $[\mathbf{L 9}$ $\left.\mathrm{Co}\left(\mathrm{CH}_{3} \mathrm{CN}\right)_{2}\right]\left(\mathrm{BF}_{4}\right)_{2} ; \mathrm{L4}-\mathrm{CoI} \bullet \mathrm{THF}$; and 3ag.

\begin{tabular}{|c|c|c|c|c|}
\hline & {$\left[\mathrm{L8Co}\left(\mathrm{CH}_{3} \mathrm{CN}\right)_{2}\right]\left(\mathrm{BF}_{4}\right)_{2}$} & {$\left[\mathrm{LPCo}\left(\mathrm{CH}_{3} \mathrm{CN}\right)_{2}\right]\left(\mathrm{BF}_{4}\right)_{2}$} & L4CoI•THF & 3ag \\
\hline Empirical formula & $\mathrm{C}_{36} \mathrm{H}_{44} \mathrm{~B}_{2} \mathrm{CoF}_{8} \mathrm{~N}_{5} \mathrm{O}_{2} \mathrm{P}$ & $\mathrm{C}_{35} \mathrm{H}_{41} \mathrm{~B}_{2} \mathrm{CoF}_{8} \mathrm{~N}_{4} \mathrm{O}_{3} \mathrm{P}$ & $\mathrm{C}_{30} \mathrm{H}_{33} \mathrm{CoIN}_{2} \mathrm{O}_{3} \mathrm{P}$ & $\mathrm{C}_{14} \mathrm{H}_{20} \mathrm{~N}_{2} \mathrm{O}$ \\
\hline Formula weight & 842.28 & 829.24 & 686.38 & 232.32 \\
\hline Crystal habit, color & Block /green & Block/green & Block /green & block/ colorless \\
\hline Crystal size, $\mathrm{mm}^{3}$ & $0.200 \times 0.180 \times 0.160$ & $0.240 \times 0.220 \times 0.200$ & $0.240 \times 0.220 \times 0.200$ & $0.220 \times 0.200 \times 0.180$ \\
\hline Temperature, $T / \mathrm{K}$ & $298(2)$ & $223(2)$ & $172(2)$ & $293(2)$ \\
\hline Wavelength, $\lambda / \AA$ & 1.54178 & 1.54178 & 1.54178 & 1.54178 \\
\hline Crystal system & monoclinic & triclinic & orthorhombic & orthorhombic \\
\hline Space group & $' P 2_{1}^{\prime}$ & 'P1' & 'P2 ${ }_{1} 2_{1} 2_{1}^{\prime}$ & 'P2 ${ }_{1} 2_{1} 2_{1}^{\prime}$ \\
\hline Unit cell dimensions & $\begin{array}{l}a=12.3483(4) \AA \\
b=11.3138(4) \AA \\
c=14.8272(4) \AA \\
\alpha=\gamma=90^{\circ} \\
\beta=99.2000(10)^{\circ}\end{array}$ & $\begin{array}{l}a=9.7764(18) \AA \\
b=10.020(2) \AA \\
c=10.928(2) \AA \\
\alpha=112.897(14)^{\circ} \\
\beta=98.386(15)^{\circ} \\
\gamma=93.96(2)^{\circ}\end{array}$ & $\begin{array}{l}a=9.4527(3) \AA \\
b=25.1917(8) \AA \\
c=27.5943(9) \AA \\
\alpha=\beta=\gamma=90^{\circ}\end{array}$ & $\begin{array}{l}a=8.3713(11) \AA \\
b=9.4586(12) \AA \\
c=17.695(3) \AA \\
\alpha=\beta=\gamma=90^{\circ}\end{array}$ \\
\hline Volume, $V / \mathrm{A}^{3}$ & 2044.81(11) & $966.2(4)$ & $6571.0(4)$ & $1401.1(3)$ \\
\hline$Z$ & 2 & 1 & 8 & 4 \\
\hline Calculated density, $\mathrm{Mg} \cdot \mathrm{m}^{-3}$ & 1.368 & 1.425 & 1.388 & 1.101 \\
\hline Absorption coefficient, $\mu / \mathrm{mm}^{-1}$ & 4.312 & 4.567 & 12.169 & 0.549 \\
\hline$F(000)$ & 870 & 427 & 2768 & 504 \\
\hline $2 \theta$ range for data collection & 3.019 to $55.983^{\circ}$ & 4.472 to $63.775^{\circ}$ & 2.375 to $68.422^{\circ}$ & 5.303 to $68.483^{\circ}$ \\
\hline Limiting indices & $\begin{array}{l}-13 \leq h \leq 13, \quad-12 \leq k \leq 12, \\
-15 \leq l \leq 15\end{array}$ & $\begin{array}{l}-11 \leq h \leq 11, \quad-11 \leq k \leq 11, \\
-12 \leq l \leq 12\end{array}$ & $\begin{array}{l}-11 \leq h \leq 11, \quad-30 \leq \\
k \leq 29,-33 \leq l \leq 33\end{array}$ & $\begin{array}{l}-10 \leq h \leq 10, \quad-9 \leq \\
k \leq 11,-20 \leq l \leq 21\end{array}$ \\
\hline Reflection collected/unique & $\begin{array}{l}13185 / 4459 \quad[R(\text { int })= \\
0.0318]\end{array}$ & $\begin{array}{l}15109 / 4841 \quad[R(\text { int })= \\
0.0310]\end{array}$ & $\begin{array}{l}65551 / 10312 \\
{[R(\text { int })=0.0776]}\end{array}$ & $\begin{array}{l}11923 / 2196 \\
{[R(\text { int })=0.0 .0418]}\end{array}$ \\
\hline Completeness to $\theta$ & $94.7 \%\left(2 \theta=55.983^{\circ}\right)$ & $98.7 \%\left(2 \theta=63.775^{\circ}\right)$ & $\begin{array}{l}99.2 \% \\
\left.68.422^{\circ}\right)\end{array} \quad(2 \theta=$ & $\begin{array}{l}99.5 \% \\
\left.68.483^{\circ}\right)\end{array} \quad(2 \theta=$ \\
\hline Max. and min. transmission & 0.545 and 0.479 & 0.7531 and 0.6060 & 0.195 and 0.158 & 0.908 and 0.889 \\
\hline Refinement method & 'SHELXL-2016/6' & 'SHELXL-2016/6' & 'SHELXL-2016/6' & 'SHELXL-2016/6' \\
\hline Data/restraints/parameters & $4459 / 1 / 504$ & $4841 / 3 / 495$ & $10312 / 2 / 690$ & $2196 / 1 / 160$ \\
\hline Goodness-of-fit on $F^{2}$ & 1.066 & 1.009 & 1.021 & 1.007 \\
\hline Final $R$ indices $[I>2 \operatorname{sigma}(I)]$ & $\begin{array}{l}R 1=0.0606, \\
w R 2=0.1829\end{array}$ & $\begin{array}{l}R 1=0.0400 \\
w R 2=0.1054\end{array}$ & $\begin{array}{l}R 1=0.0555 \\
w R 2=0.1446\end{array}$ & $\begin{array}{l}R 1=0.0517 \\
w R 2=0.1463\end{array}$ \\
\hline$R$ indices (all data) & $\begin{array}{l}R 1=0.0630 \\
w R 2=0.1850\end{array}$ & $\begin{array}{l}R 1=0.0420 \\
w R 2=0.1100\end{array}$ & $\begin{array}{l}R 1=0.0663 \\
w R 2=0.1531\end{array}$ & $\begin{array}{l}R 1=0.0606 \\
w R 2=0.1586\end{array}$ \\
\hline Largest diff. peak and hole & 0.973 and $--0.329 \mathrm{e} \cdot \AA^{-3}$ & 0.482 and $-0.298 \mathrm{e} \cdot \AA^{-3}$ & $\begin{array}{l}1.018 \text { and }-0.977 \\
\mathrm{e} \cdot \AA^{-3}\end{array}$ & $\begin{array}{l}0.250 \text { and }-0.236 \\
\text { e. } \AA^{-3}\end{array}$ \\
\hline
\end{tabular}




\section{Procedure of Co-Catalyzed AAA Reaction with $\left[\mathrm{LBCo}\left(\mathrm{CH}_{3} \mathrm{CN}\right)_{2}\right]\left(\mathrm{BF}_{4}\right)_{2}$ and L4-CoI:}

Procedure of Co-Catalyzed AAA Reaction with $\left[\mathbf{L 8}-\mathrm{Co}\left(\mathrm{CH}_{3} \mathrm{CN}\right)_{2}\right]\left(\mathrm{BF}_{4}\right)_{2}$

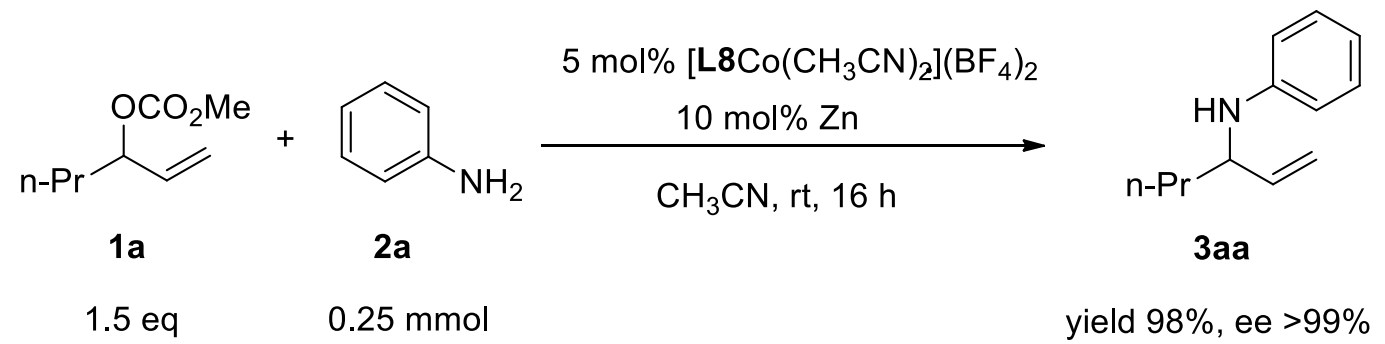

A reaction tube equipped with magnetic stir bar was charged with $5 \mathrm{~mol} \%$ of $\left[\mathbf{L 8 C o}\left(\mathbf{C H}_{3} \mathbf{C N}\right)_{2}\right]\left(\mathbf{B F}_{4}\right)_{2}(10.6$ $\left.\mathrm{mg}, 1.3 \times 10^{-2} \mathrm{mmol}, 0.05 \mathrm{eq}\right)$ and $10 \mathrm{~mol} \%$ of $\mathrm{Zn}$ dust $\left(1.7 \mathrm{mg}, 2.6 \times 10^{-2} \mathrm{mmol}, 0.10 \mathrm{eq}\right)$ in $2 \mathrm{~mL}$ of acetonitrile and stirred for 20 minutes in an $\mathrm{N}_{2}$ filled glove box. Then, $1 \mathrm{a}(60.0 \mathrm{mg}, 0.38 \mathrm{mmol}, 1.5 \mathrm{eq})$ and $\mathbf{2 a}(23.5 \mathrm{mg}$, $0.25 \mathrm{mmol}, 1 \mathrm{eq})$ were added sequentially to the reaction mixture. The reaction tube was sealed and brought out from the glove box. The reaction mixture was stirred for 16 hours at room temperature. The solvent was removed and the residue was purified by flash column chromatography with $25 \%$ toluene/PE as the eluent.

\section{Procedure of Co-Catalyzed AAA Reaction with L4-CoI}

To a reaction tube equipped with magnetic stir bar was charged with $5 \mathrm{~mol} \%$ of $\mathbf{L 8}-\mathbf{C o I}\left(9 \mathrm{mg}, 1.3 \times 10^{-2}\right.$ mmol, $0.05 \mathrm{eq})$ in $2 \mathrm{~mL}$ of acetonitrile were added $\mathbf{1 a}(60.0 \mathrm{mg}, 0.38 \mathrm{mmol}, 1.5 \mathrm{eq})$ and $\mathbf{2 b}(23.5 \mathrm{mg}, 0.25$ mmol, 1 eq) and stirred for 20 minutes in an $\mathrm{N}_{2}$ filled glove box. Followed by addition of different additive, the reaction mixture was stirred for 16 hours at room temperature. The solvent was removed and the residue was purified by flash column chromatography with $25 \%$ toluene/PE as the eluent. 
Optimization with L8-CoI•THF catalyst:

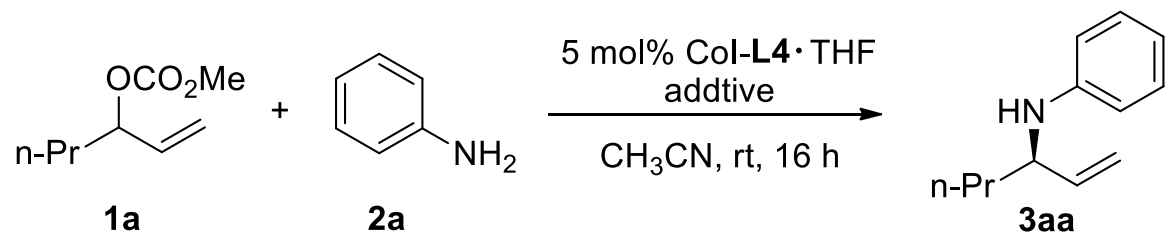

$1.5 \mathrm{eq} \quad 0.25 \mathrm{mmol}$

\begin{tabular}{|c|c|c|c|}
\hline Entry & Additive & Yield & $e e$ \\
\hline 1. & -- & $\mathrm{Nr}$ & - \\
\hline 2. & $\operatorname{AgOTf}(5 \mathrm{~mol} \%)$ & $<5 \%$ & - \\
\hline 3. & $\mathrm{AgBF}_{4}(5 \mathrm{~mol} \%)$ & $<5 \%$ & - \\
\hline \multirow[t]{2}{*}{4.} & $\operatorname{AgOTf}(5 \mathrm{~mol} \%) /$ & $46 \%$ & nd \\
\hline & $\mathrm{Zn}\left(\mathrm{BF}_{4}\right)_{2}(5 \mathrm{~mol} \%)$ & & \\
\hline \multirow[t]{2}{*}{5.} & $\mathrm{AgBF}_{4}(5 \mathrm{~mol} \%) /$ & $<5$ & - \\
\hline & $\mathrm{Zn}(\mathrm{OTf})_{2}(5 \mathrm{~mol} \%)$ & & \\
\hline 6. & NaBARF (20 mol\%) & - & - \\
\hline 7. & $\left(\mathrm{C}_{6} \mathrm{~F}_{5}\right)_{3} \mathrm{~B}(20 \mathrm{~mol} \%)$ & - & - \\
\hline \multirow[t]{2}{*}{8.} & NaBARF $(20 \mathrm{~mol} \%) /$ & - & - \\
\hline & $\mathrm{La}(\mathrm{OTf})_{3}(20 \mathrm{~mol} \%)$ & & \\
\hline 9. & $\mathrm{Zn}\left(\mathrm{BF}_{4}\right)_{2}(5 \mathrm{~mol} \%)$ & $68 \%$ & $96.0 \%$ \\
\hline 10. & $\mathrm{Zn}\left(\mathrm{BF}_{4}\right)_{2}(10 \mathrm{~mol} \%)$ & $84 \%$ & $99.0 \%$ \\
\hline 11. & $\mathrm{Zn}\left(\mathrm{BF}_{4}\right)_{2}(15 \mathrm{~mol} \%)$ & $82 \%$ & $98.5 \%$ \\
\hline 12. & $\mathrm{Zn}\left(\mathrm{BF}_{4}\right)_{2}(25 \mathrm{~mol} \%)$ & $60 \%$ & $98.5 \%$ \\
\hline
\end{tabular}




\section{NMR Spectra of new compounds:}

[1f]<smiles>C=CC(OC(=O)OC)C1CC1</smiles>

[1f]<smiles>C=CC(OC(=O)OC)C1CC1</smiles>

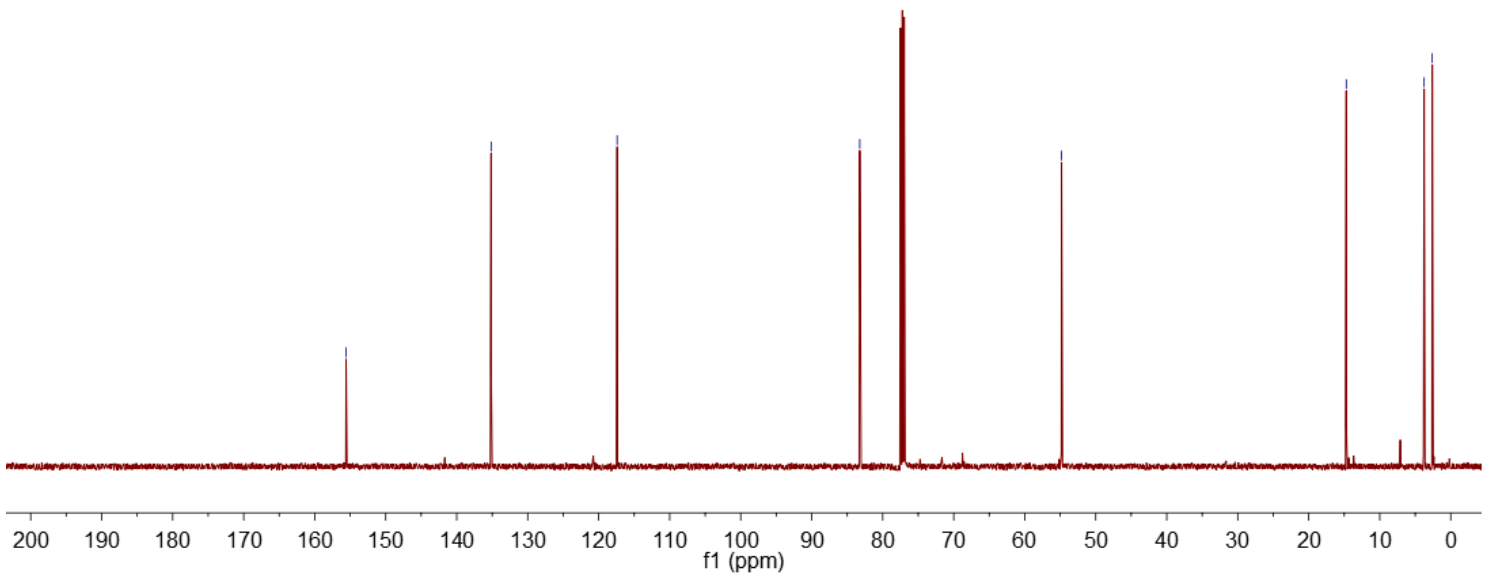


[4]

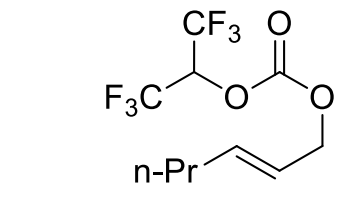

[4]

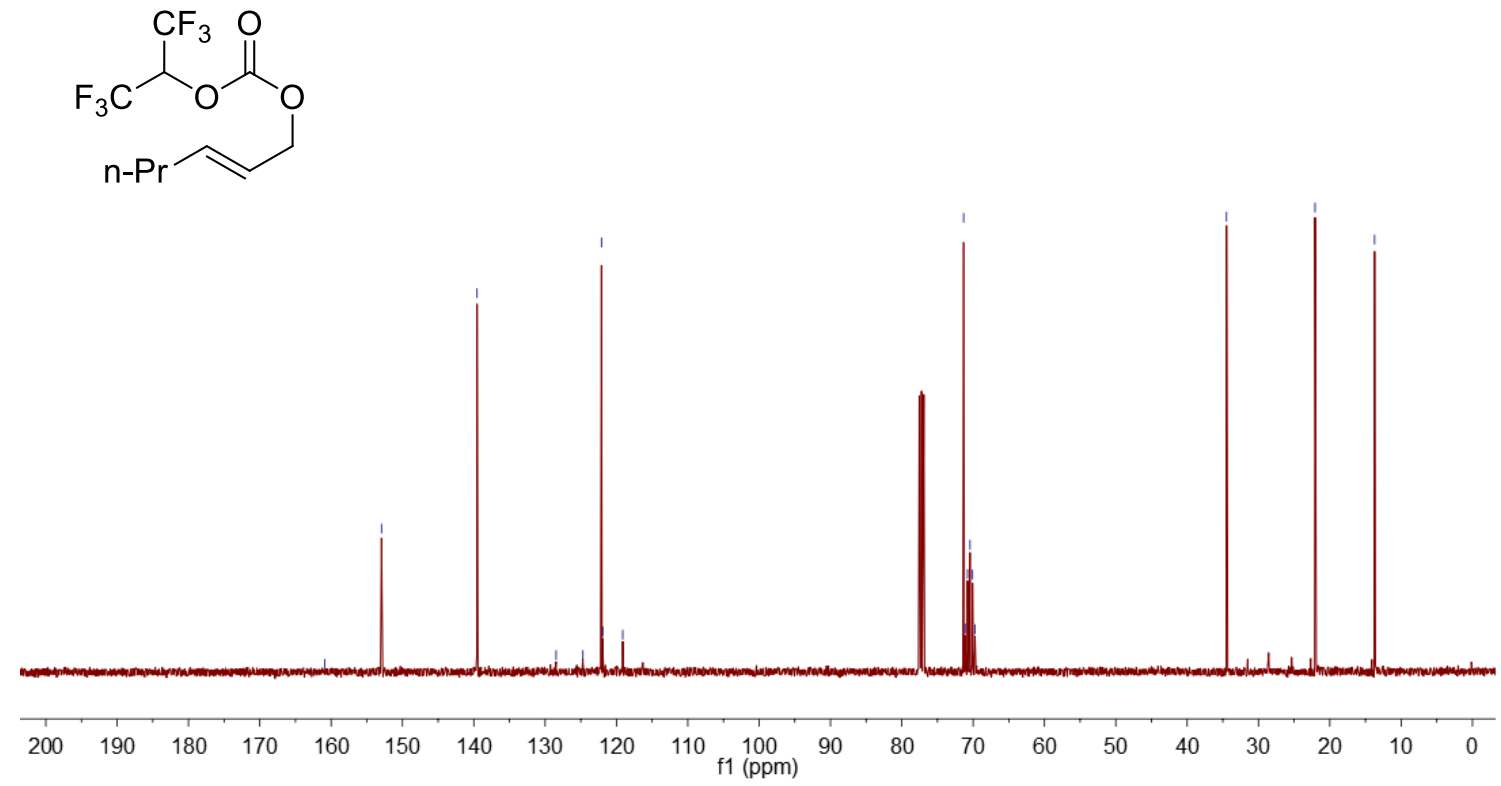


[5]<smiles>O=C(OC/C=C\PP)OC(C(F)(F)F)C(F)(F)F</smiles>

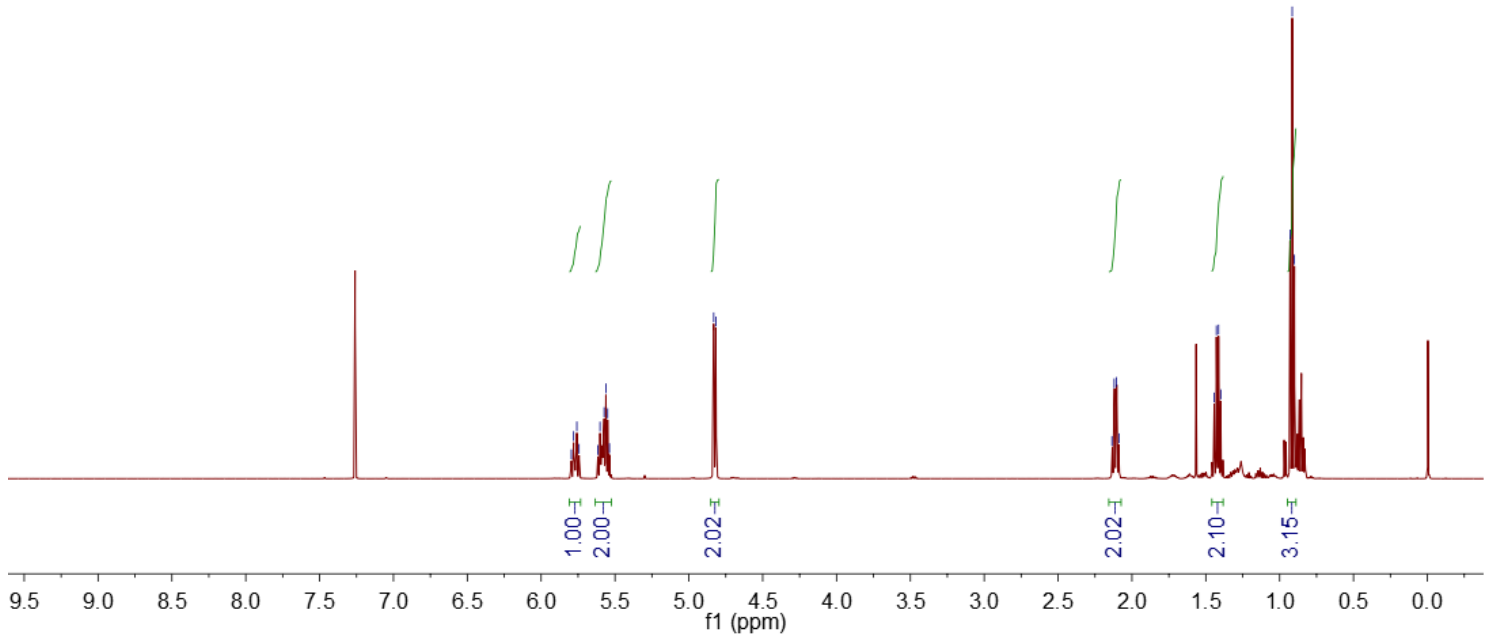

[5]<smiles>O=C(OC/C=C\[PH])OC(C(F)(F)F)C(F)(F)F</smiles>

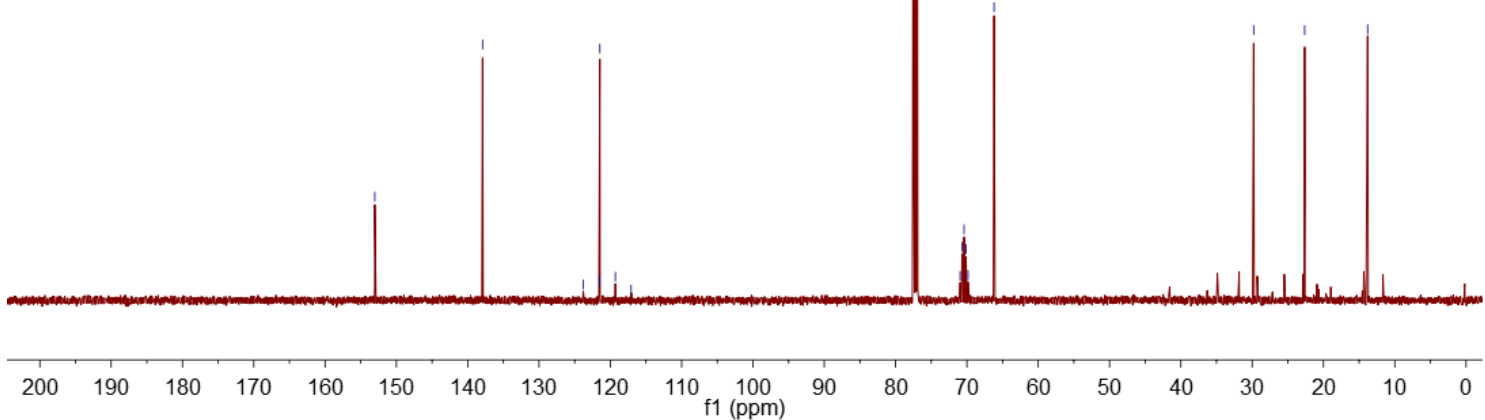




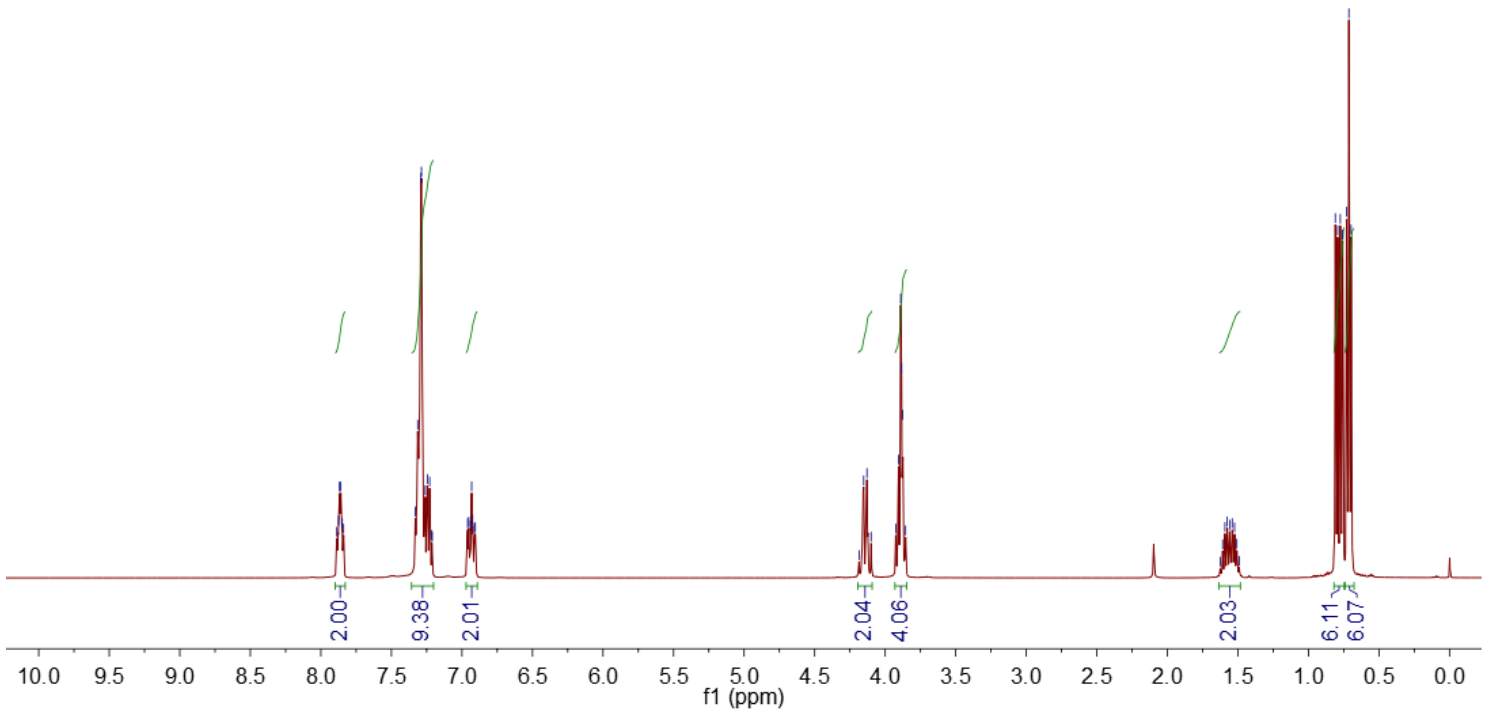

[L2]

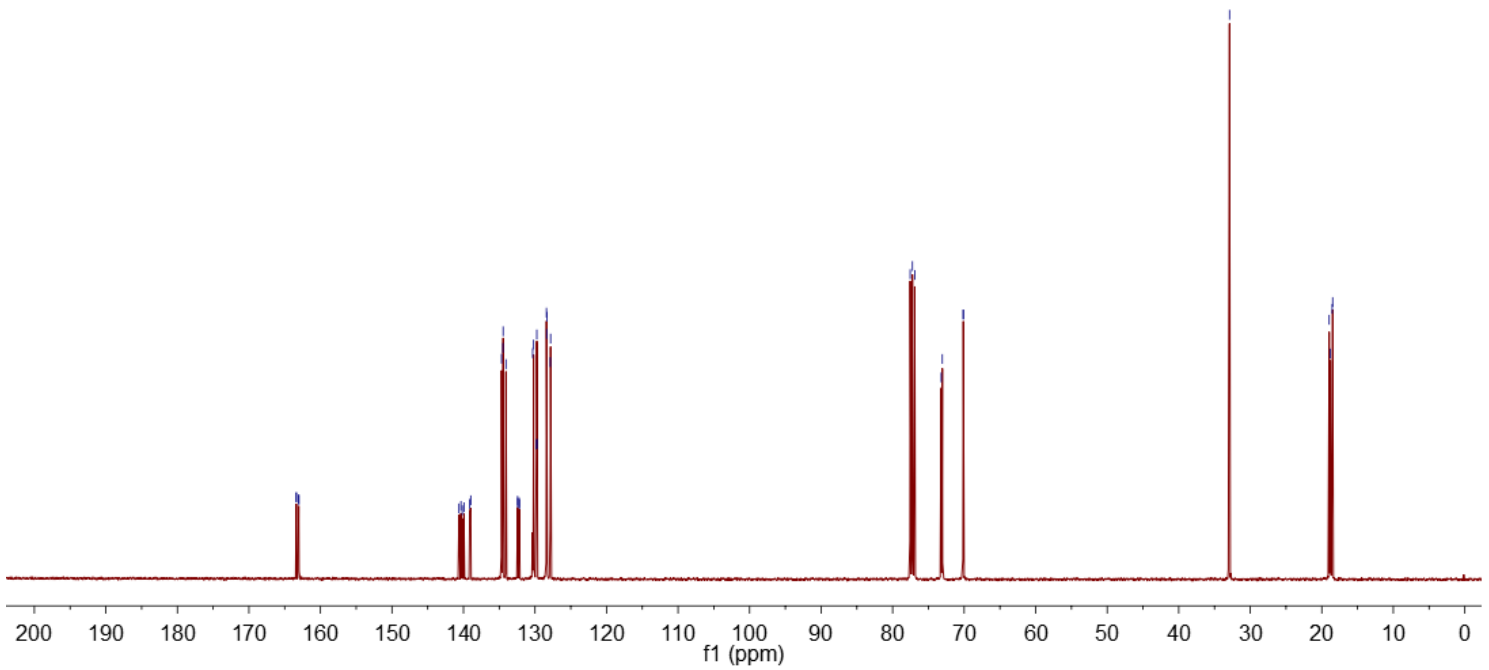




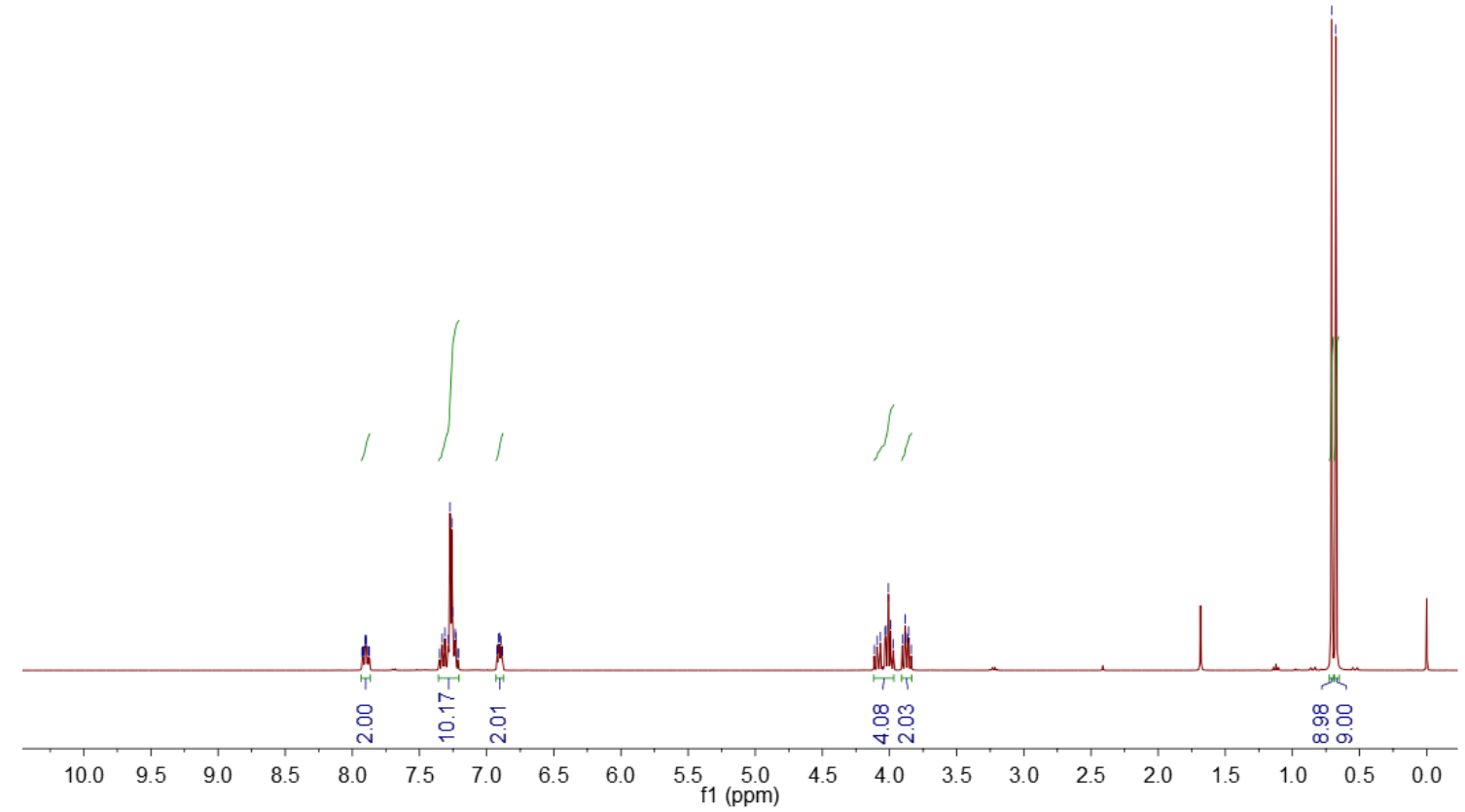

[L3] MmN

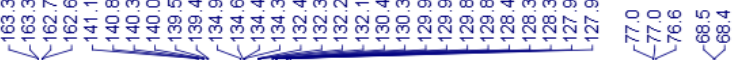

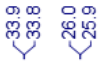

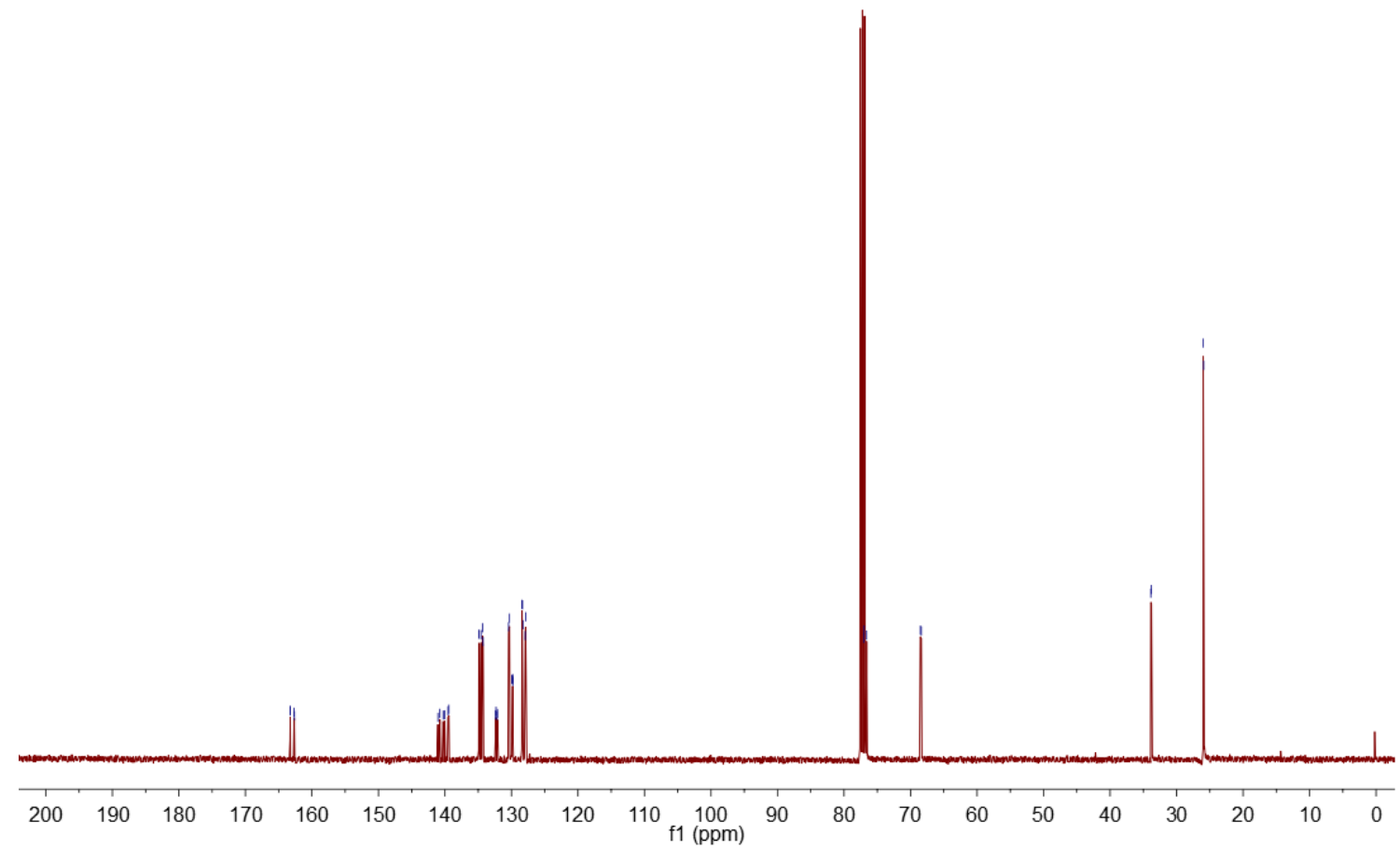




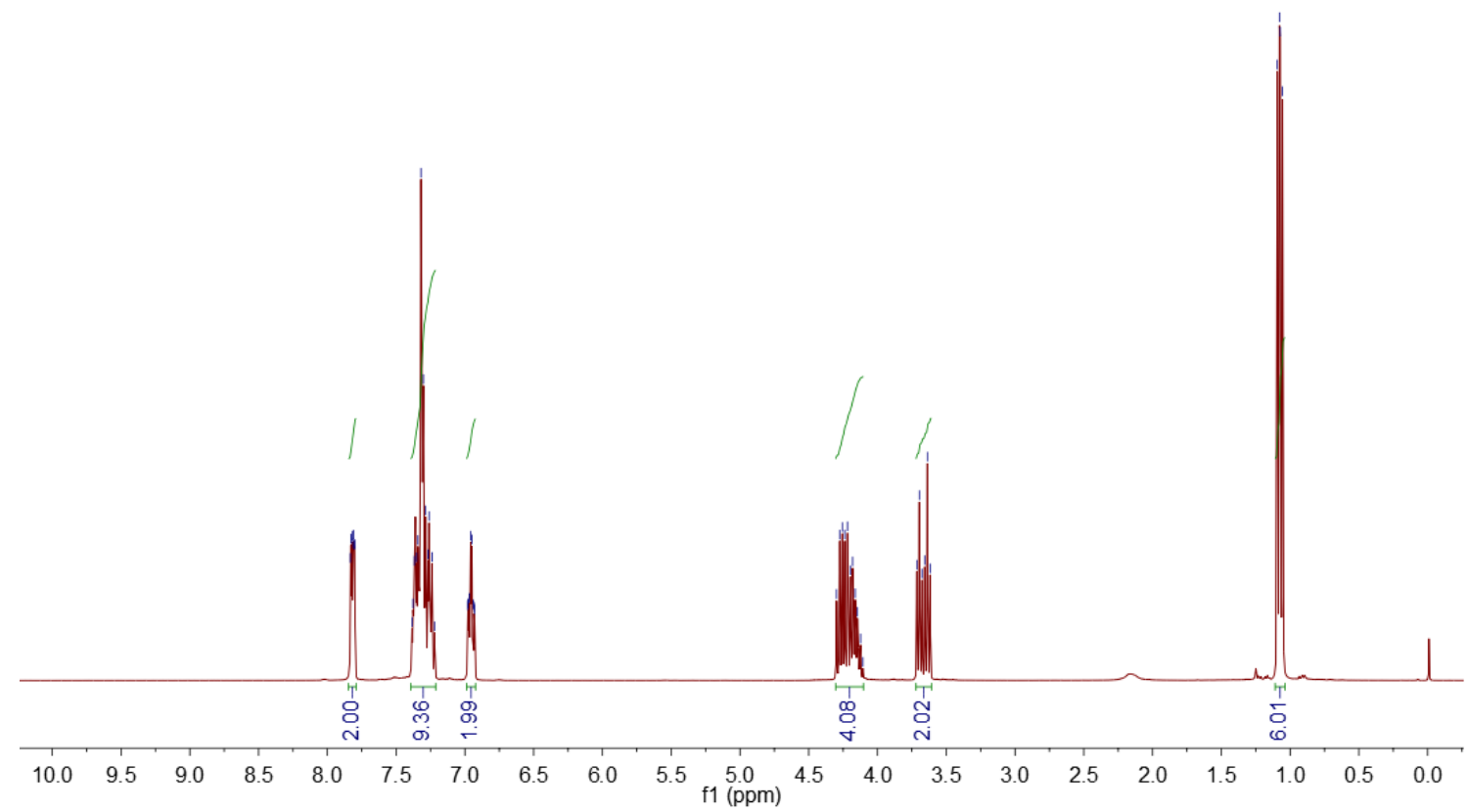

[L4]

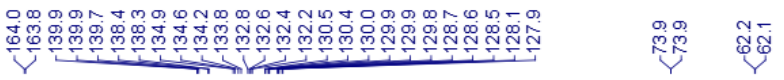

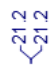

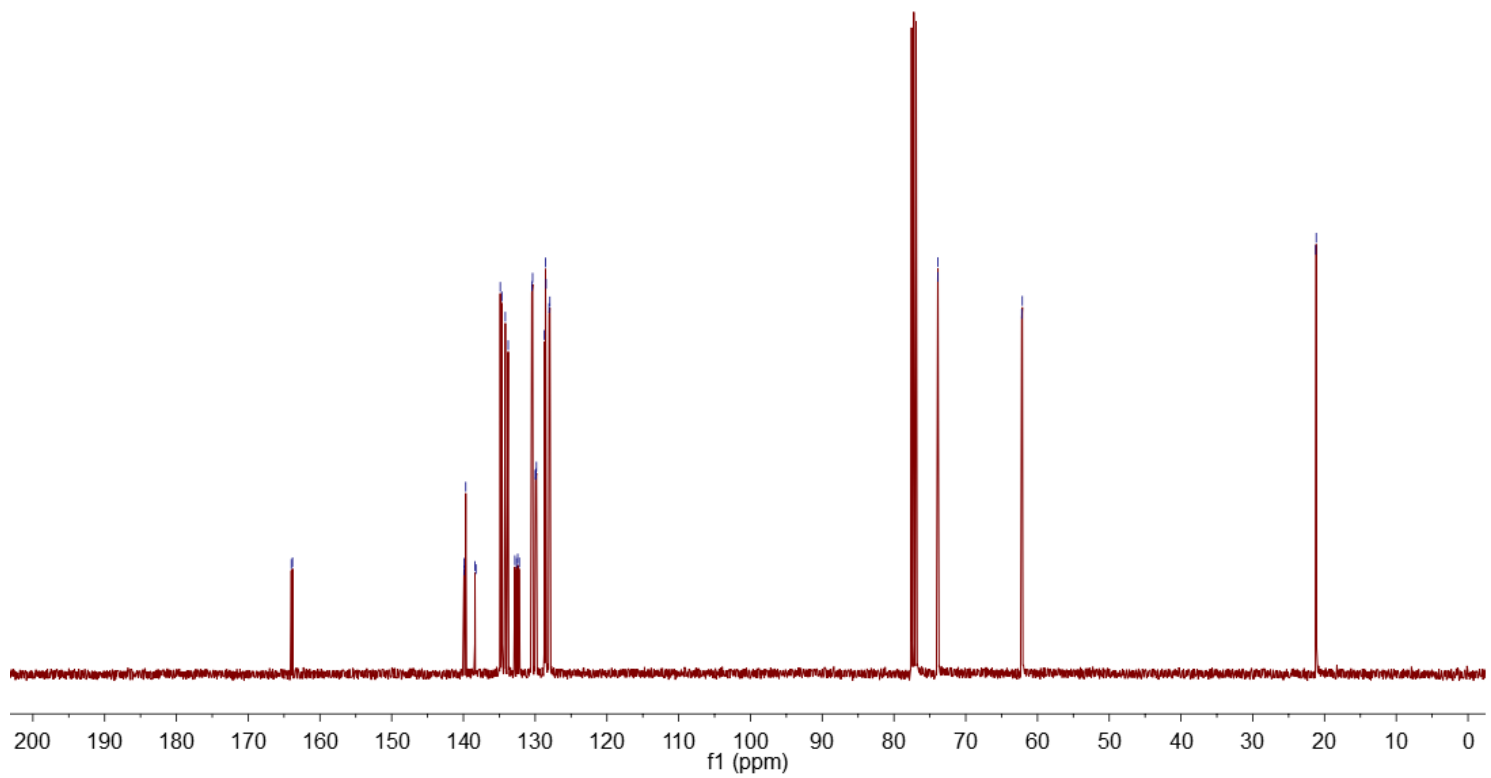




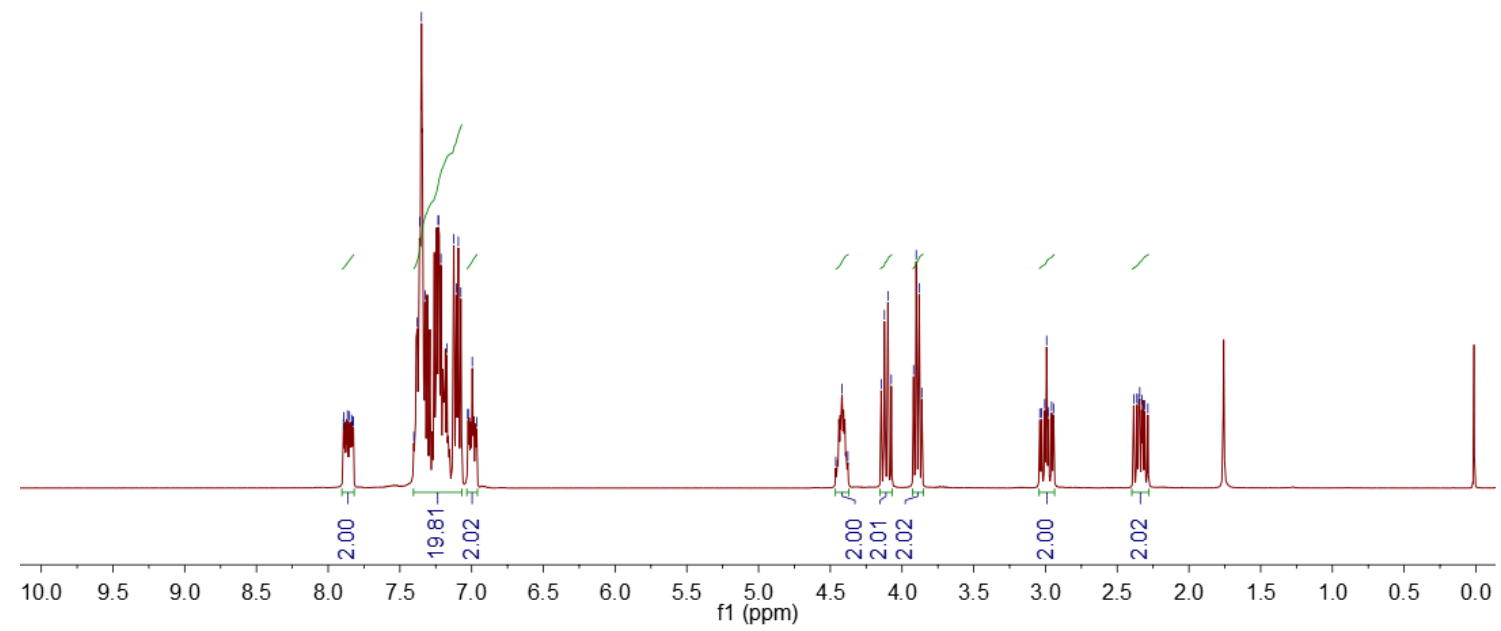

[L5]

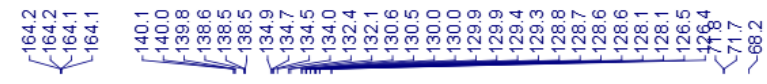

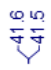

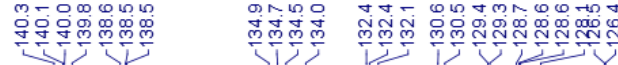
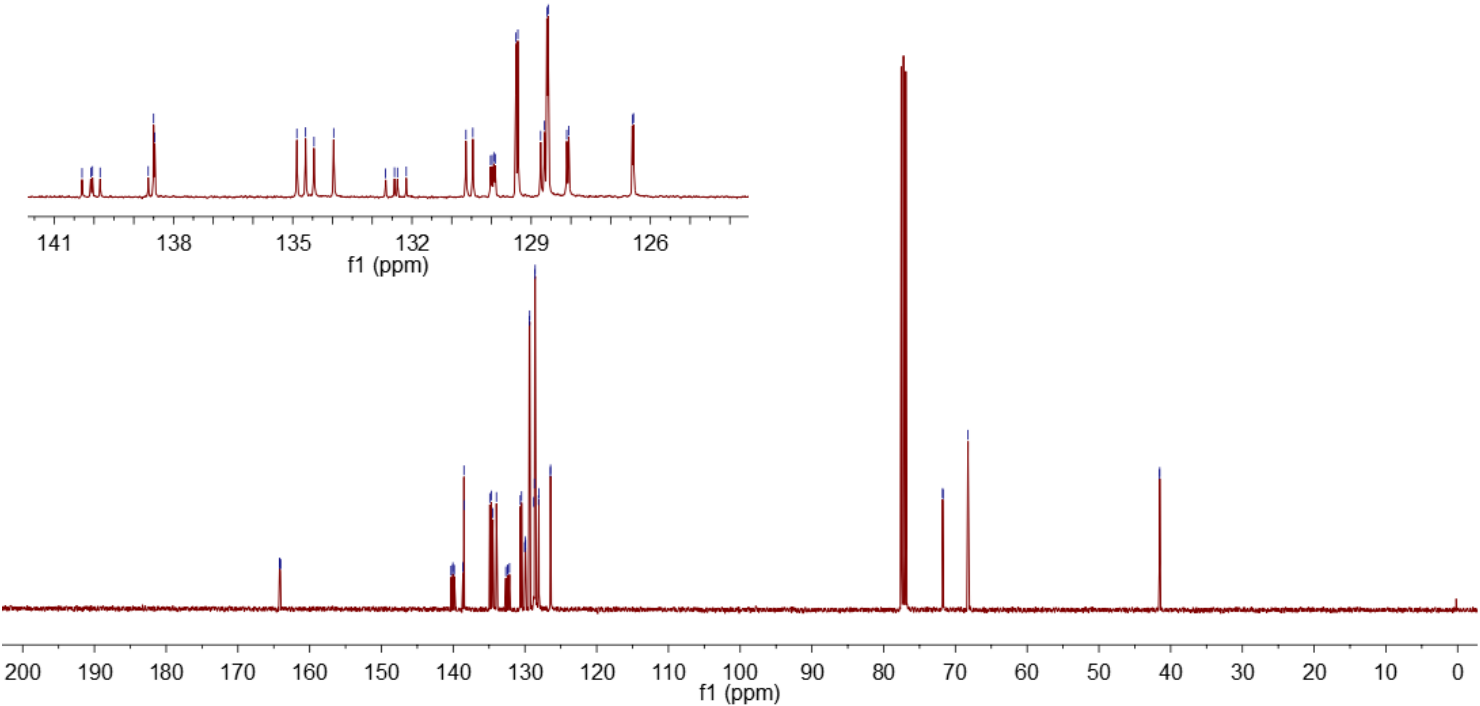

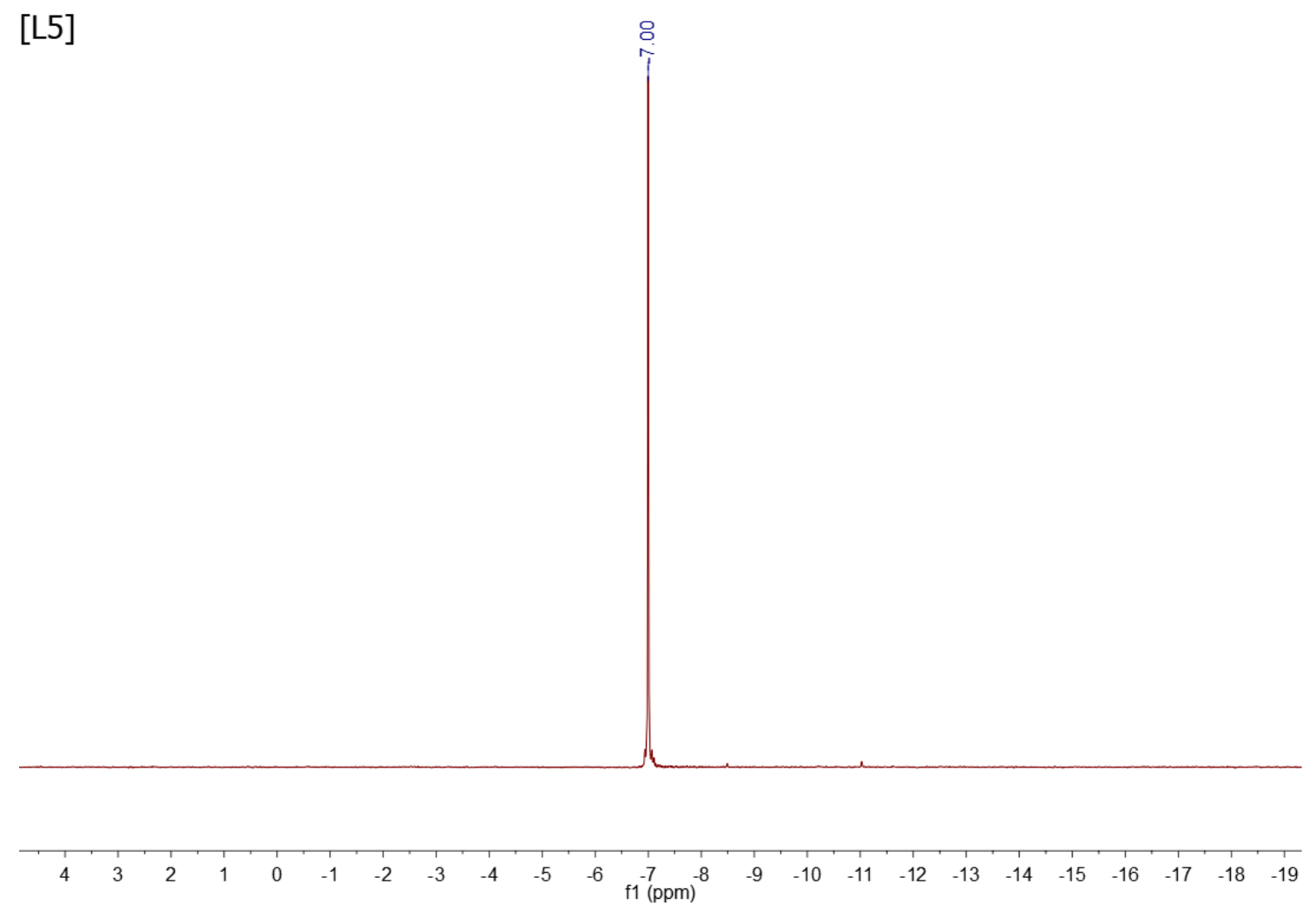

[L6]

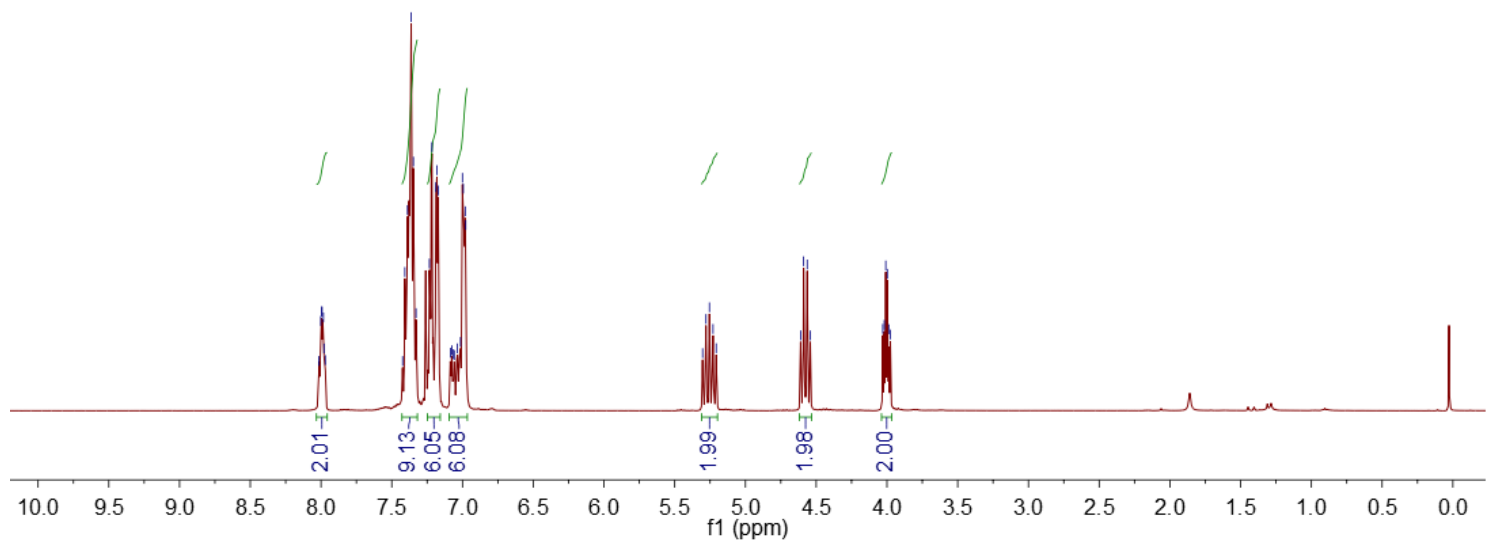




\section{[L6]}

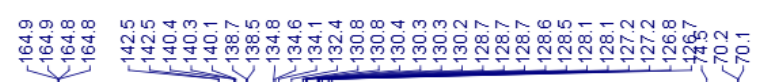

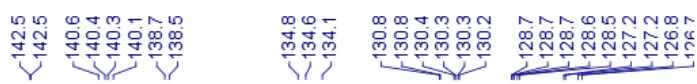
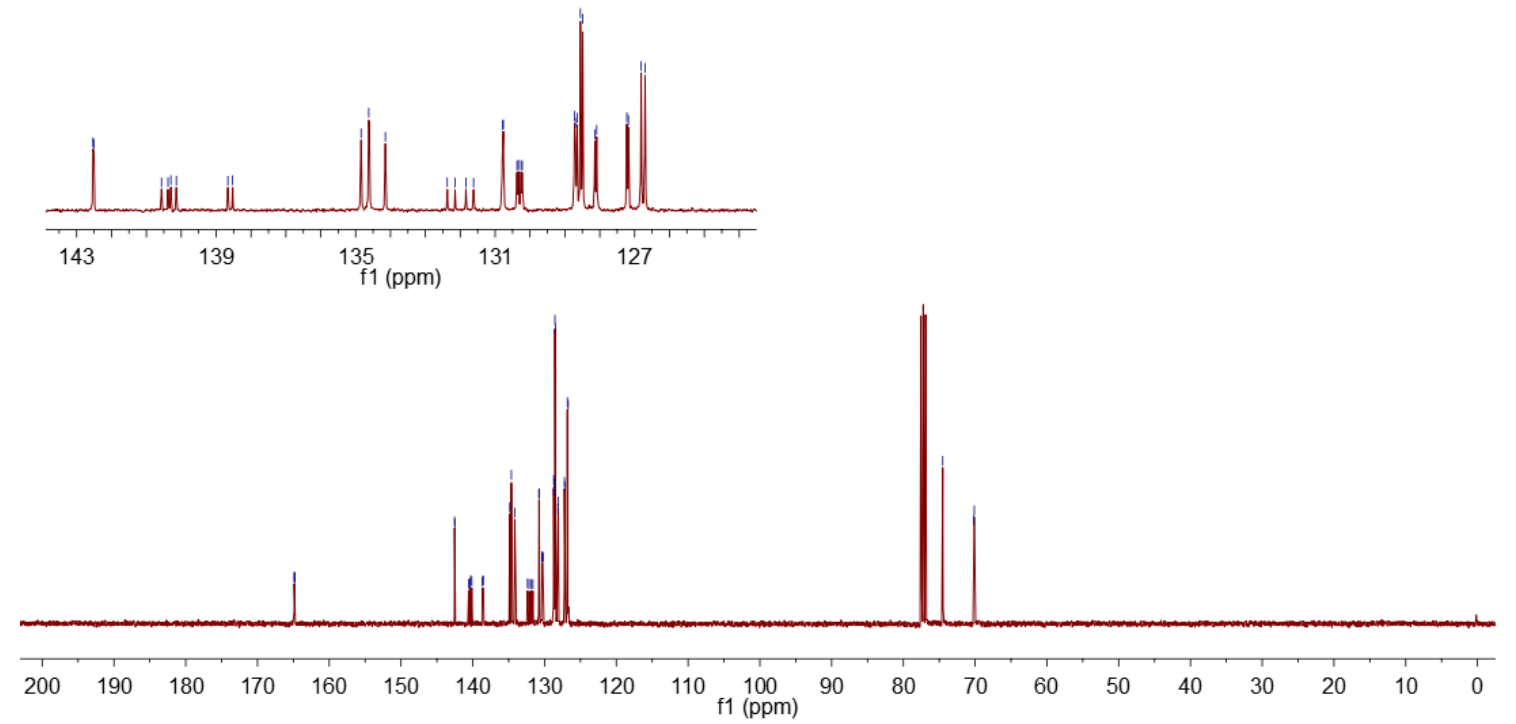

[L6] 


\section{[L7]}

๙

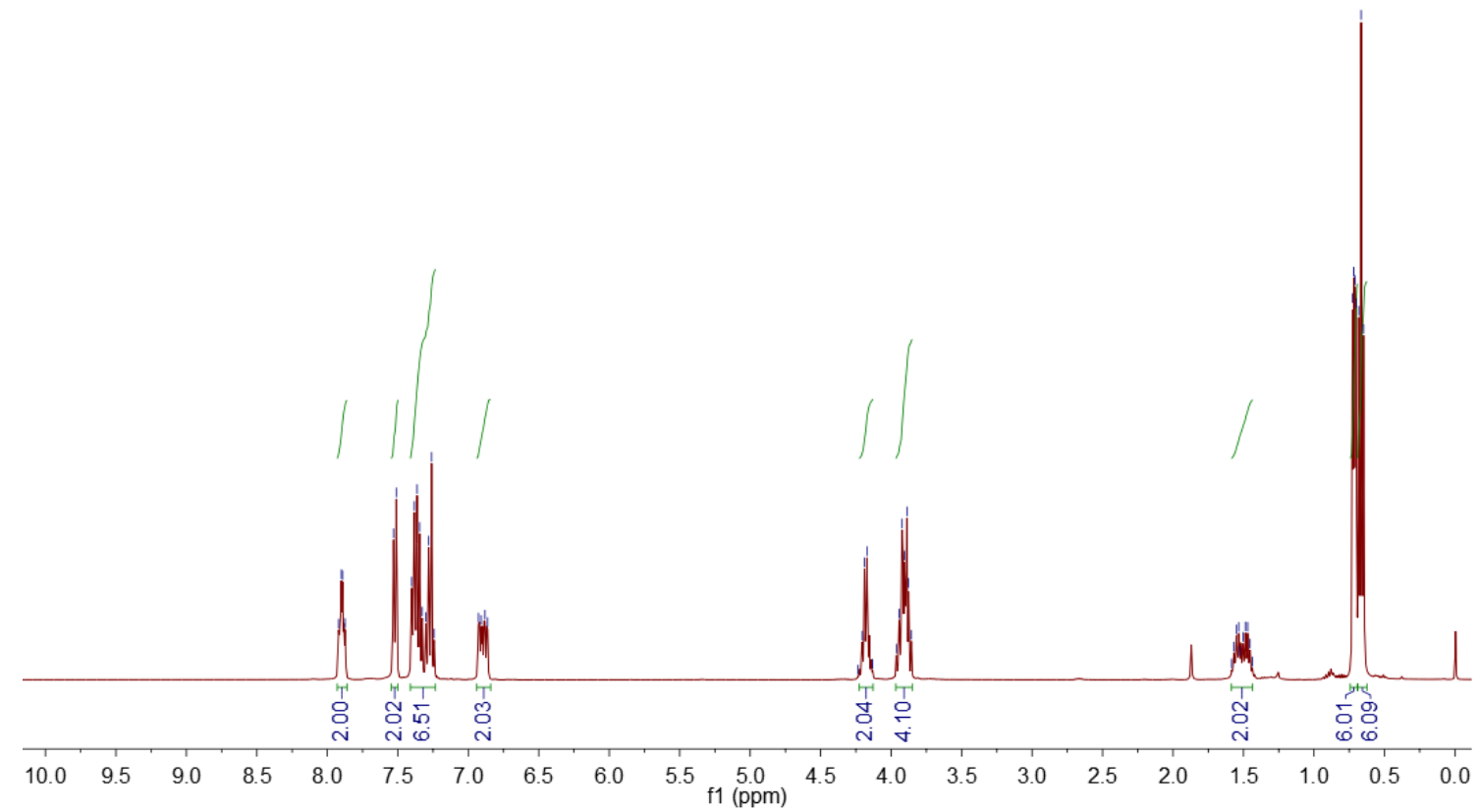

[L7]

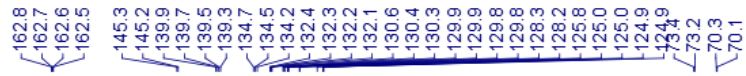

赵

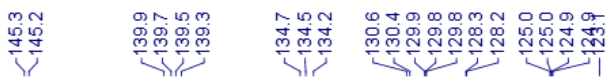
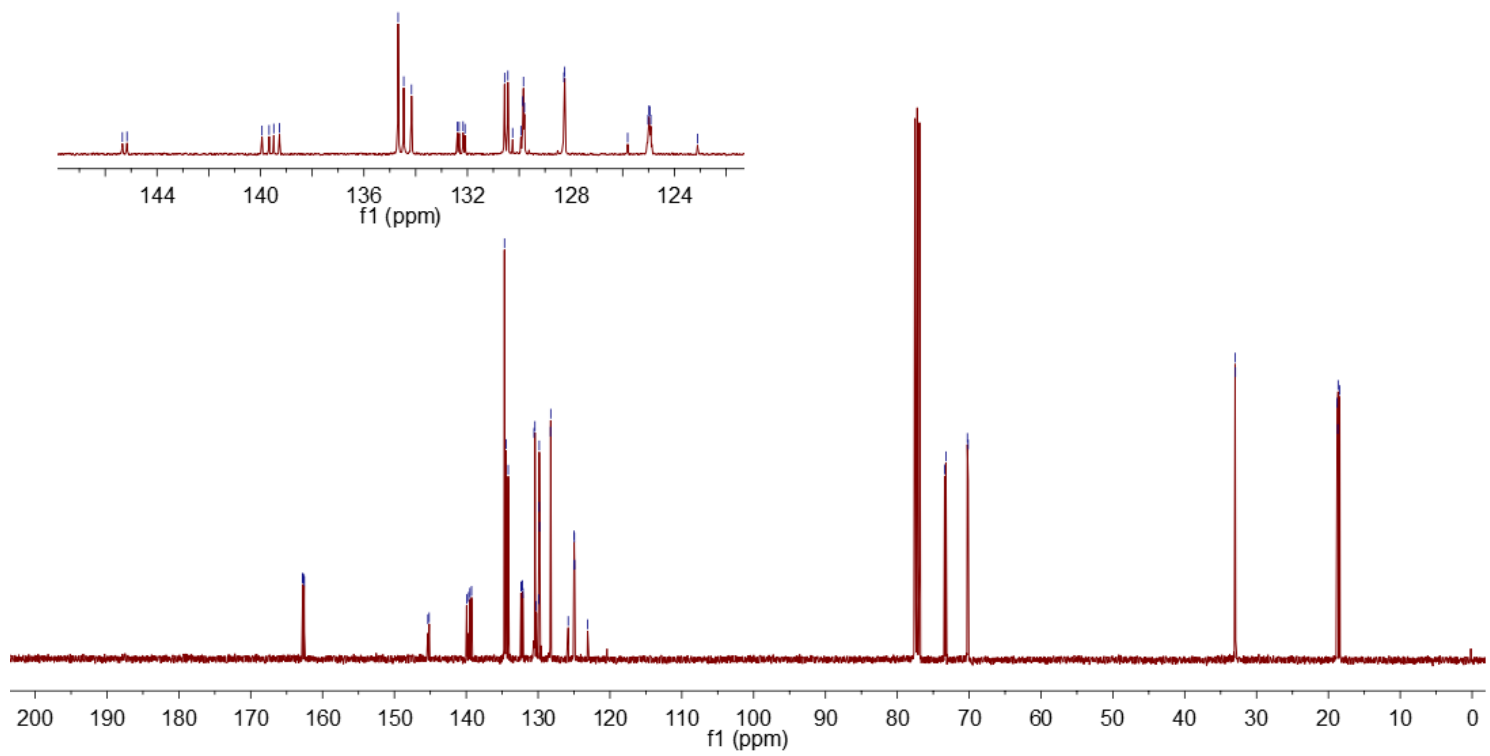
[L7]

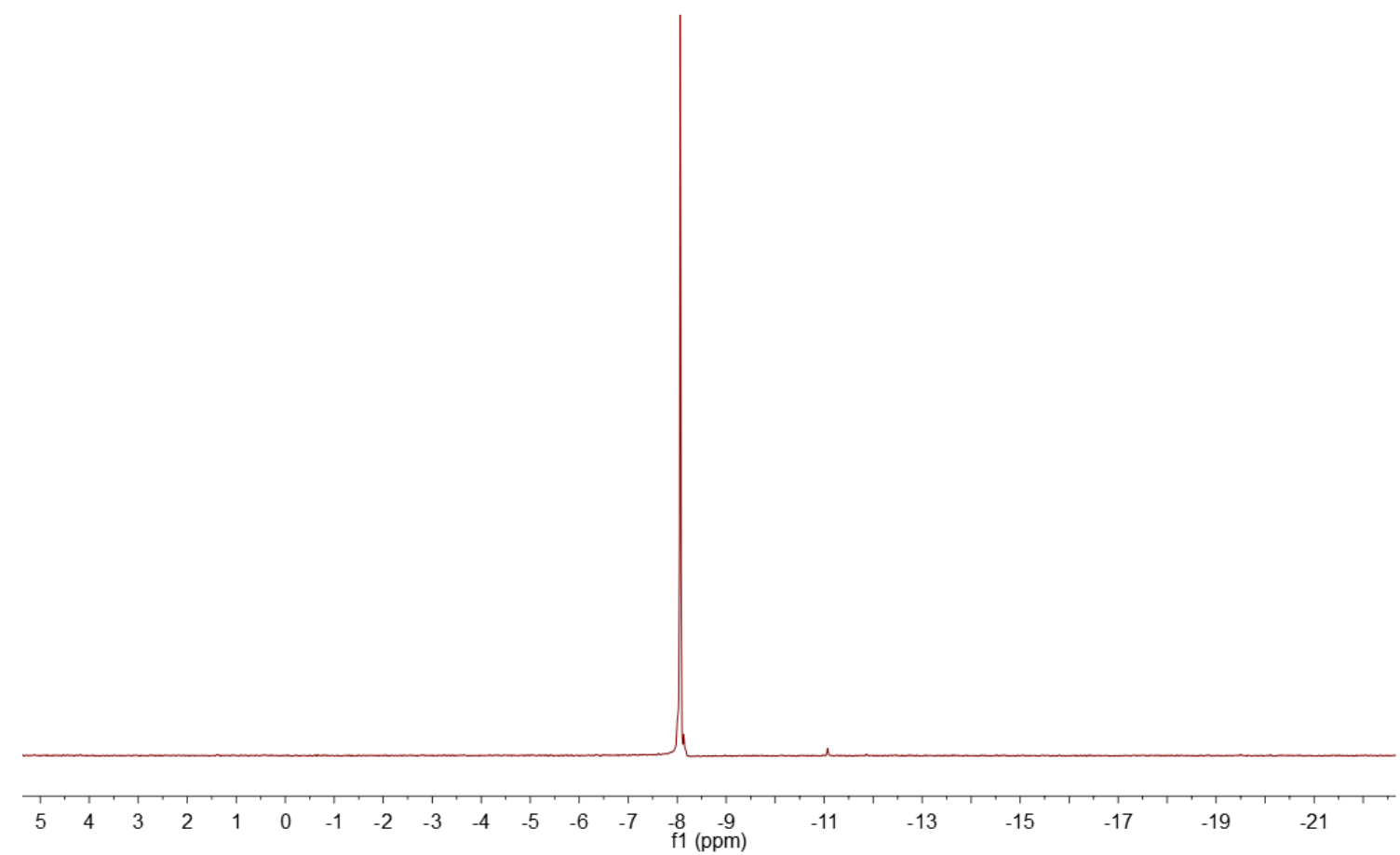

[L8]
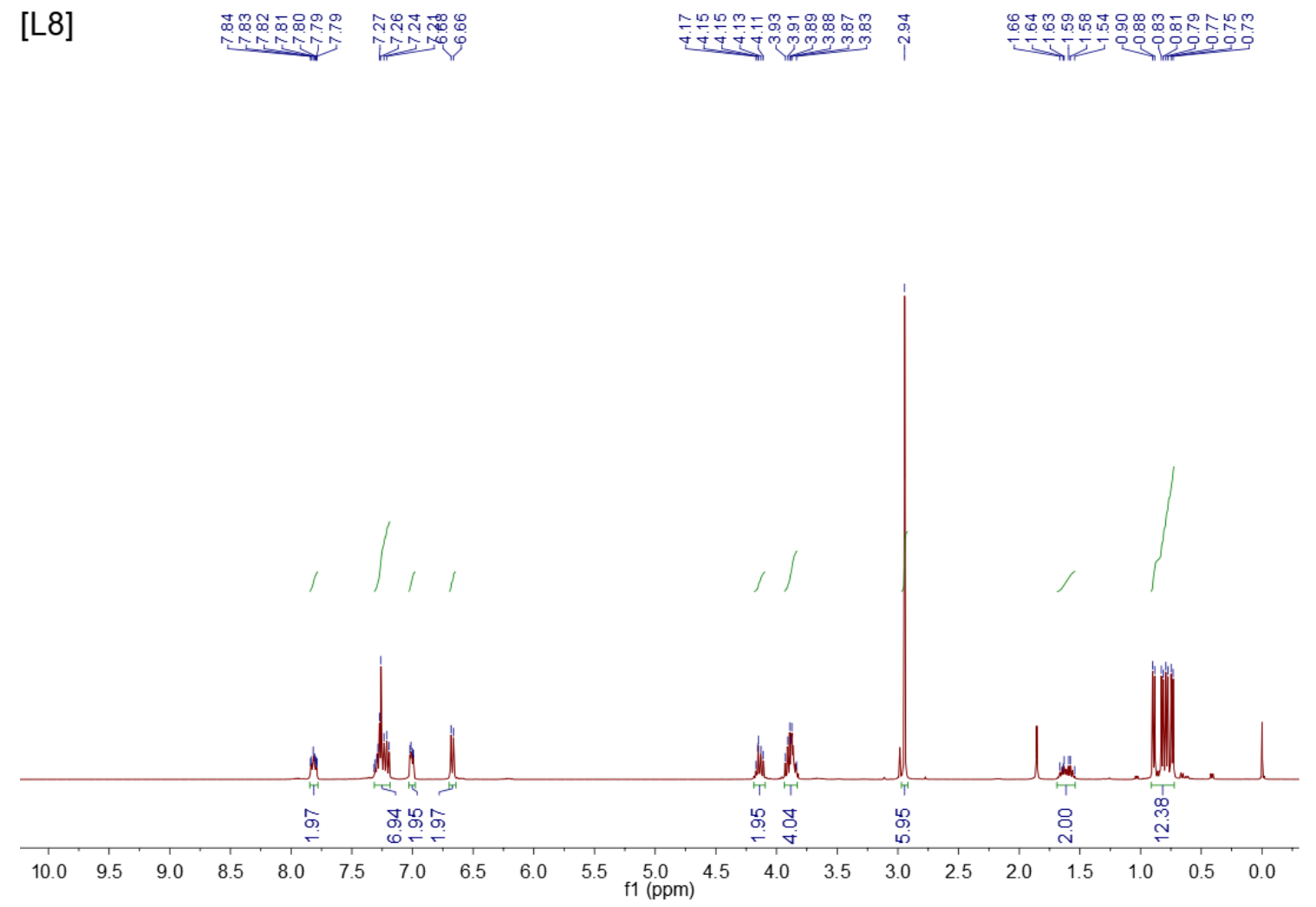


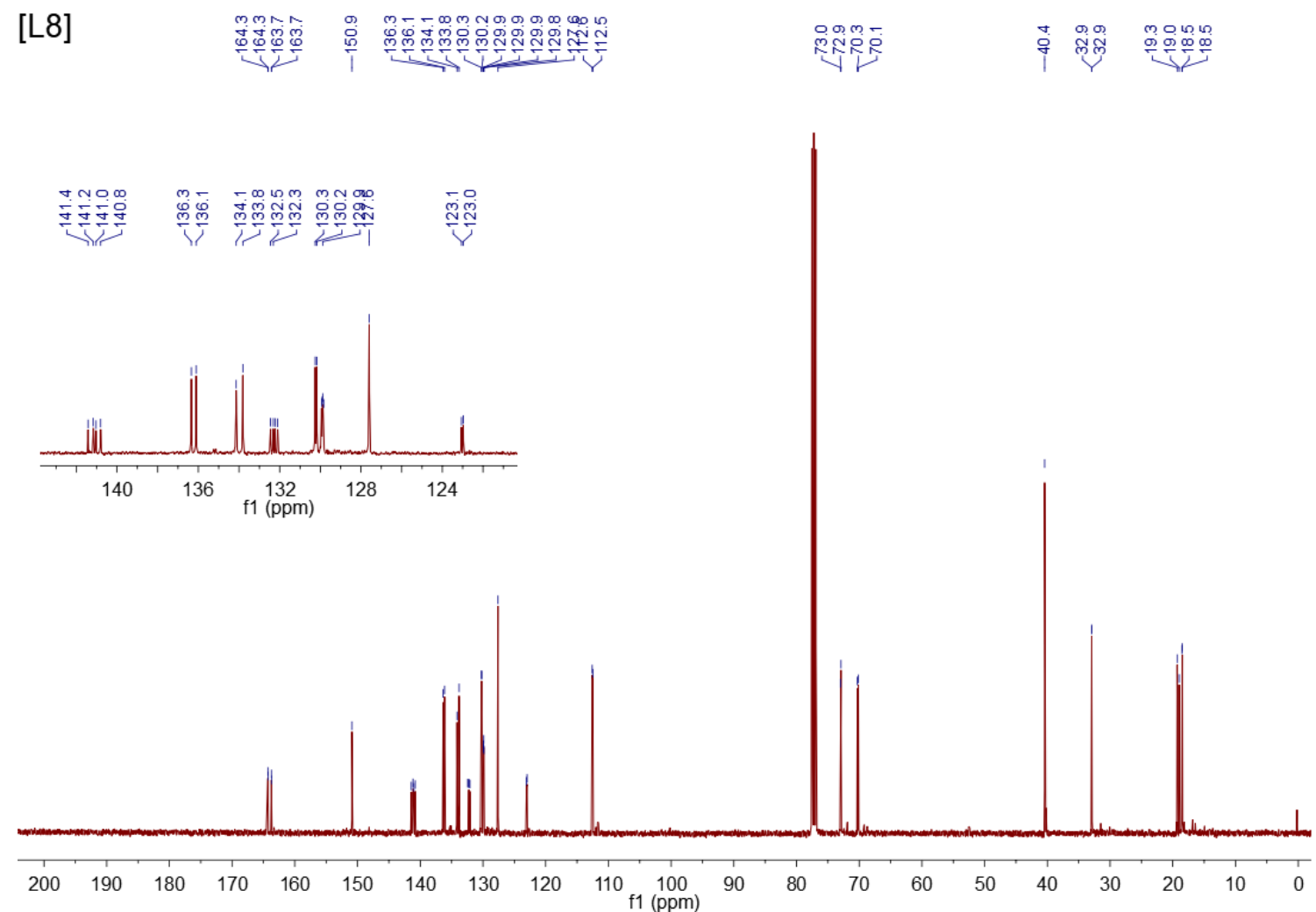

\section{[L8]}

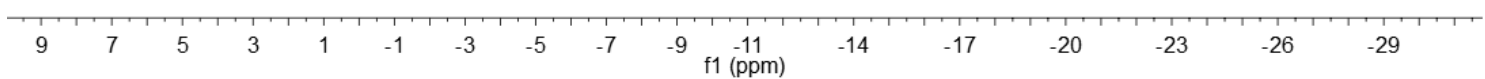




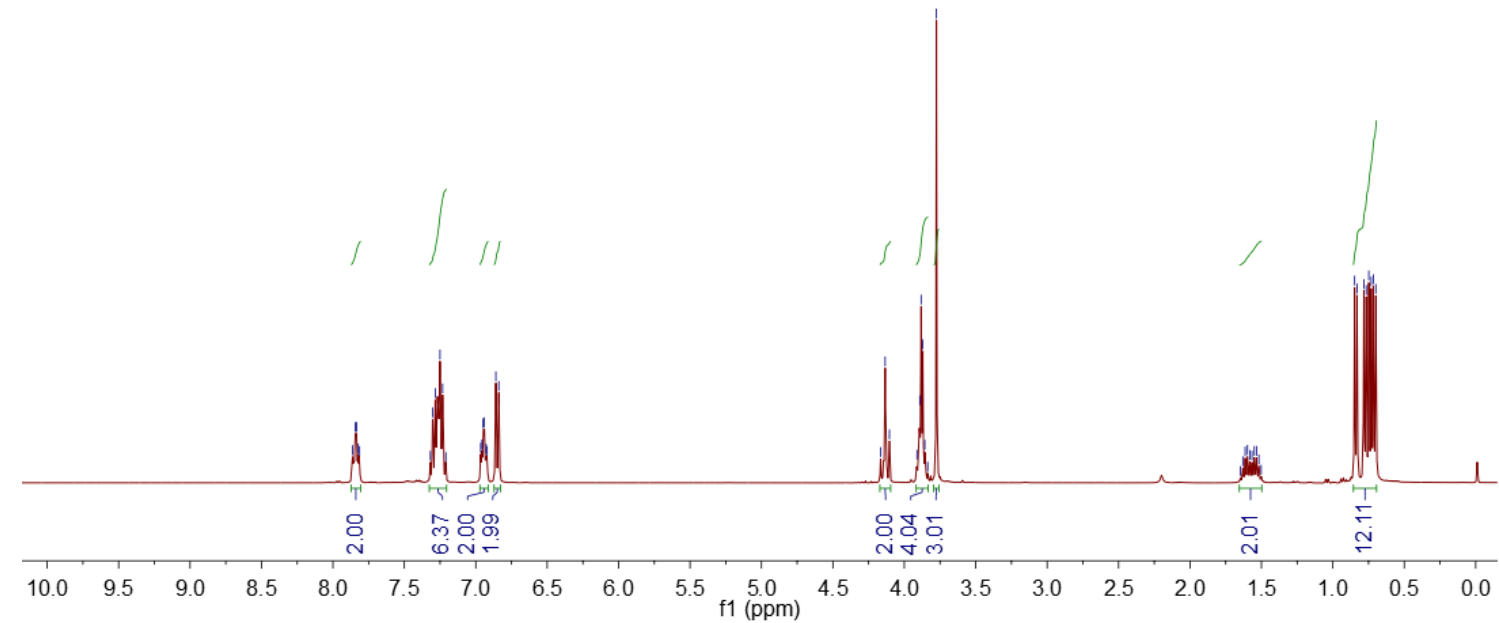

[L9]

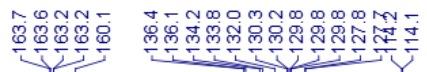
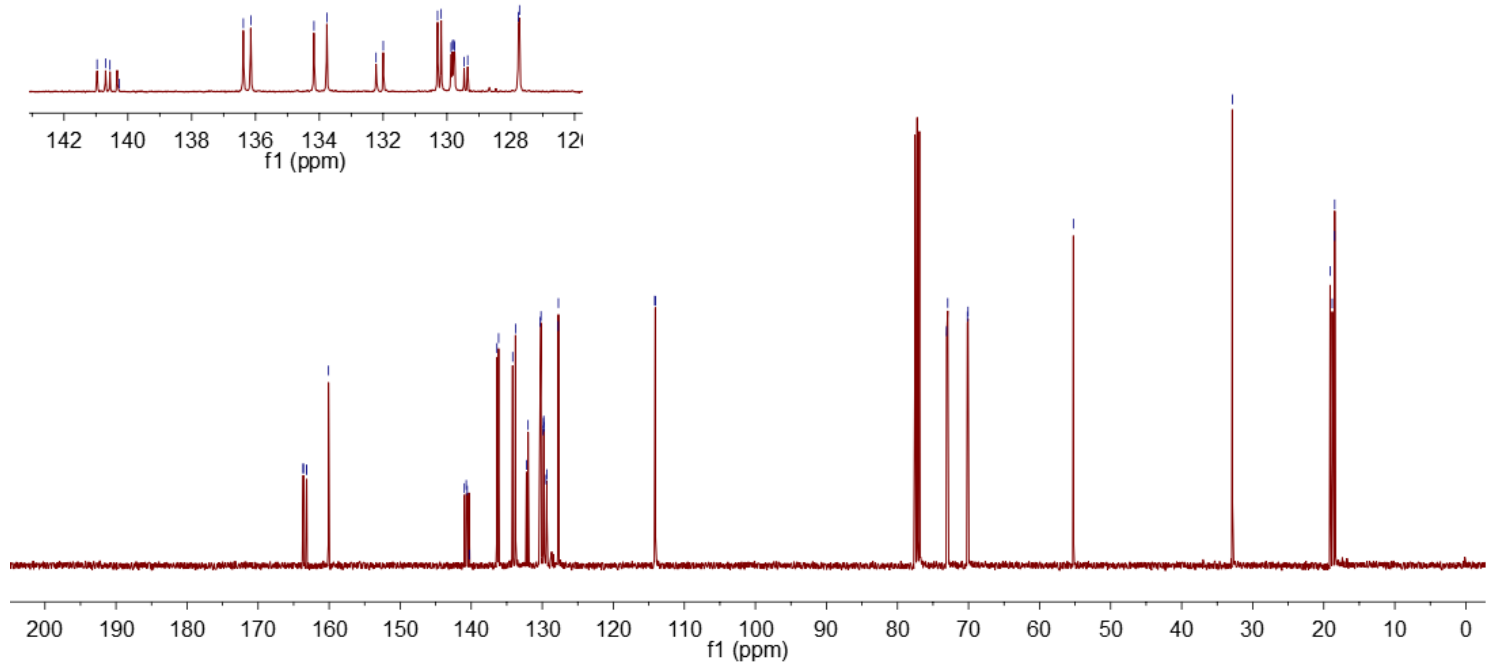


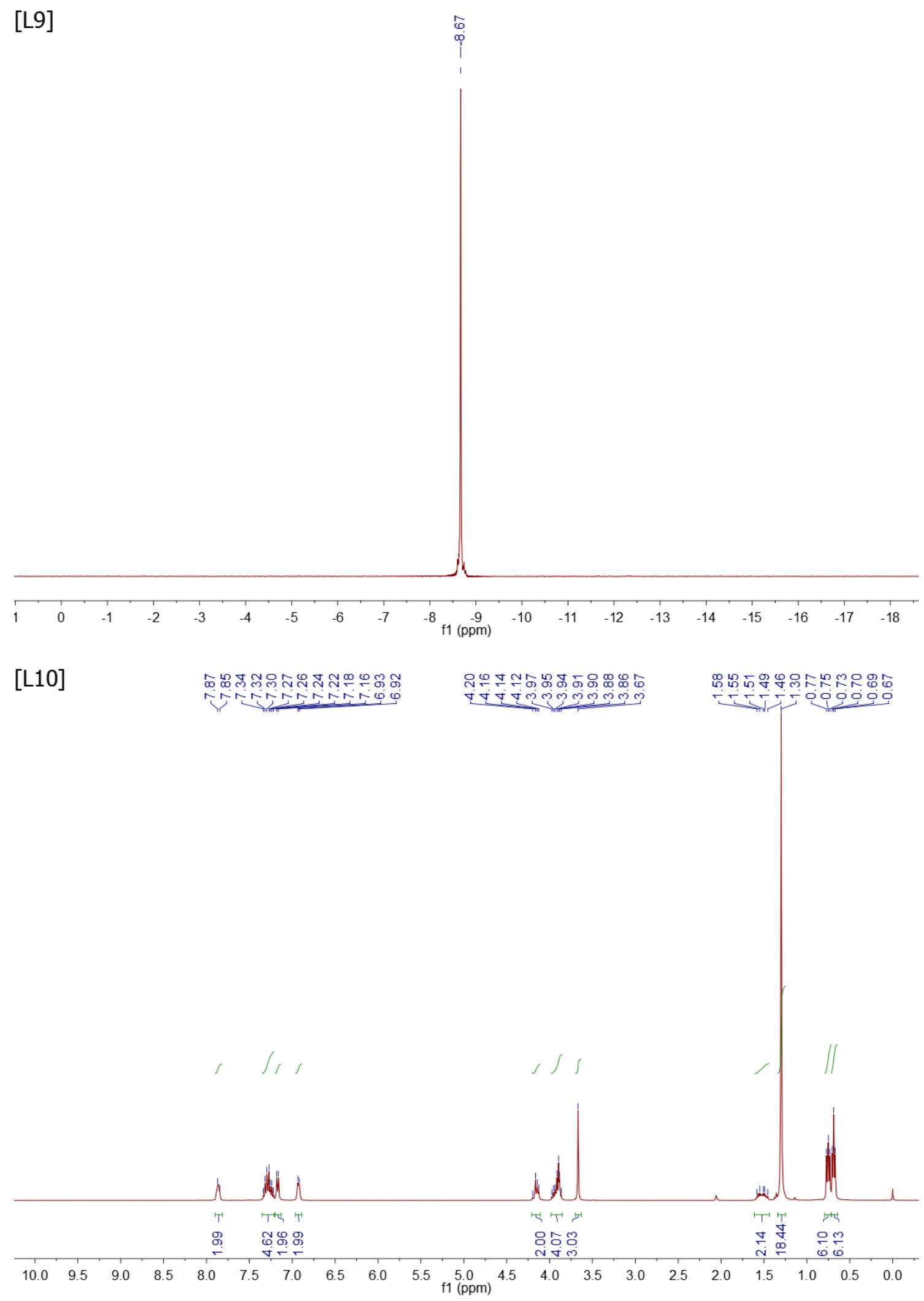


[L10]
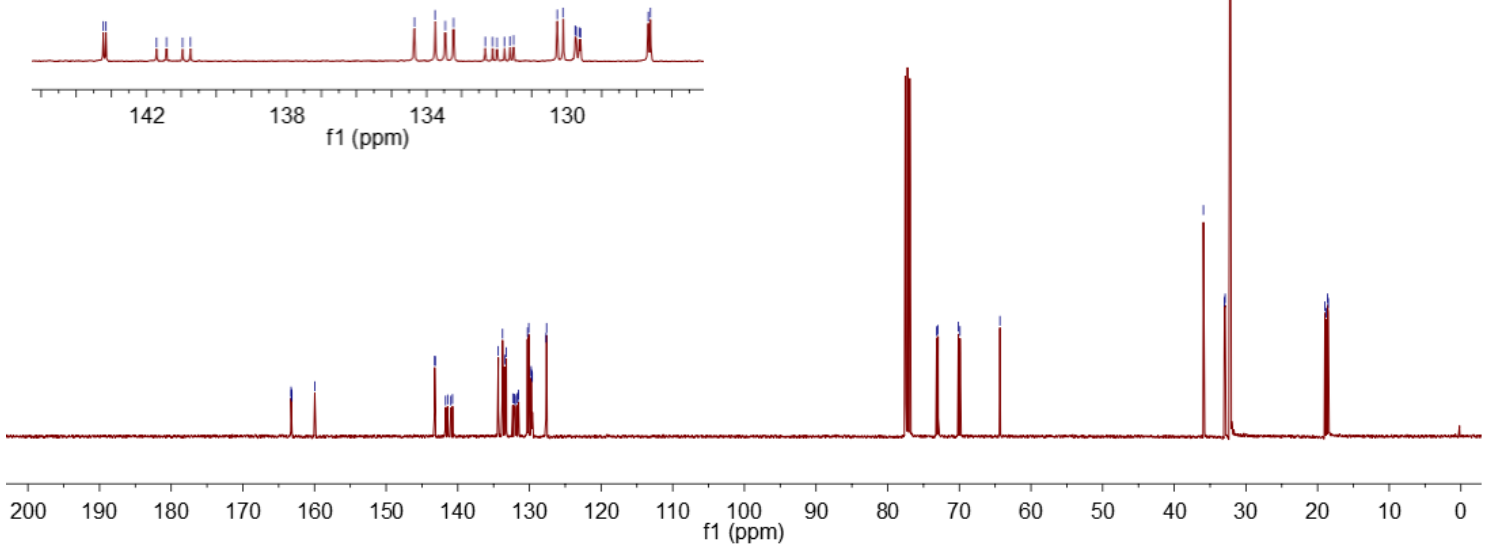

[L10]

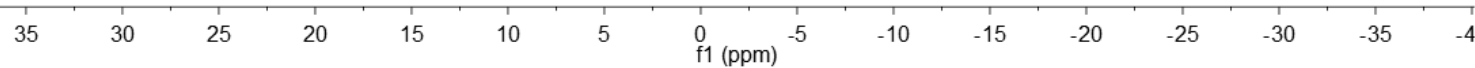




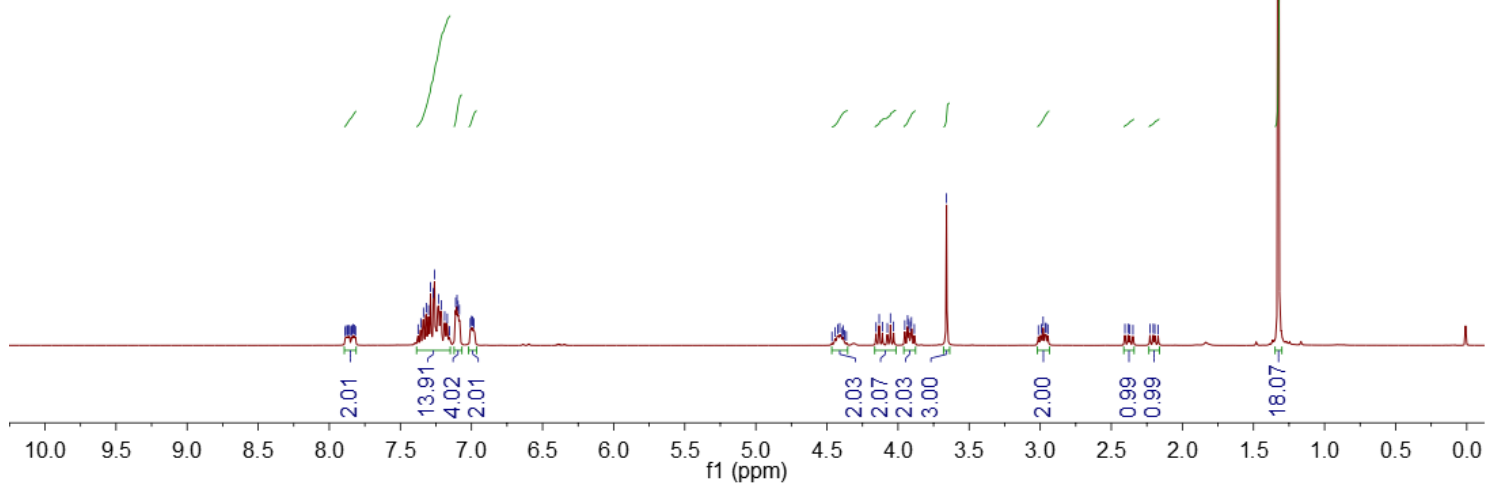

[L11]

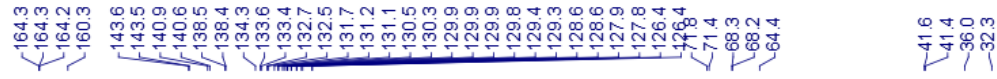

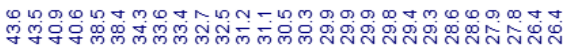

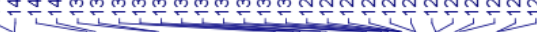
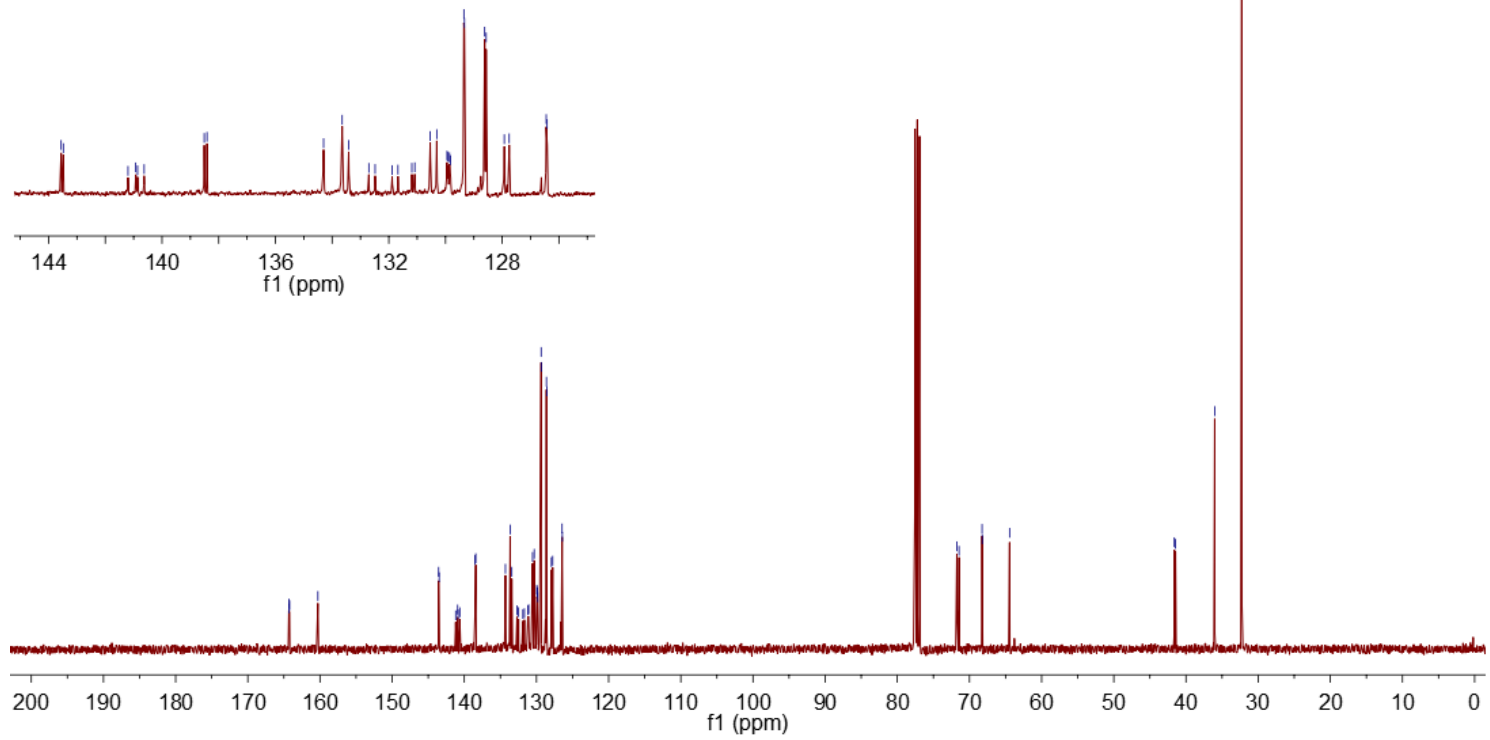
[L11]

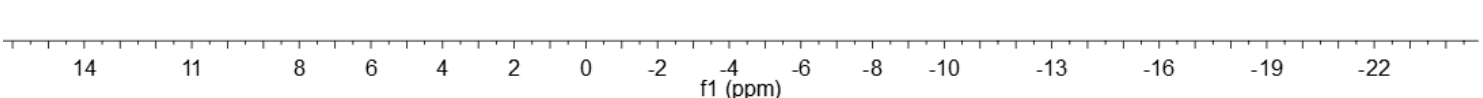




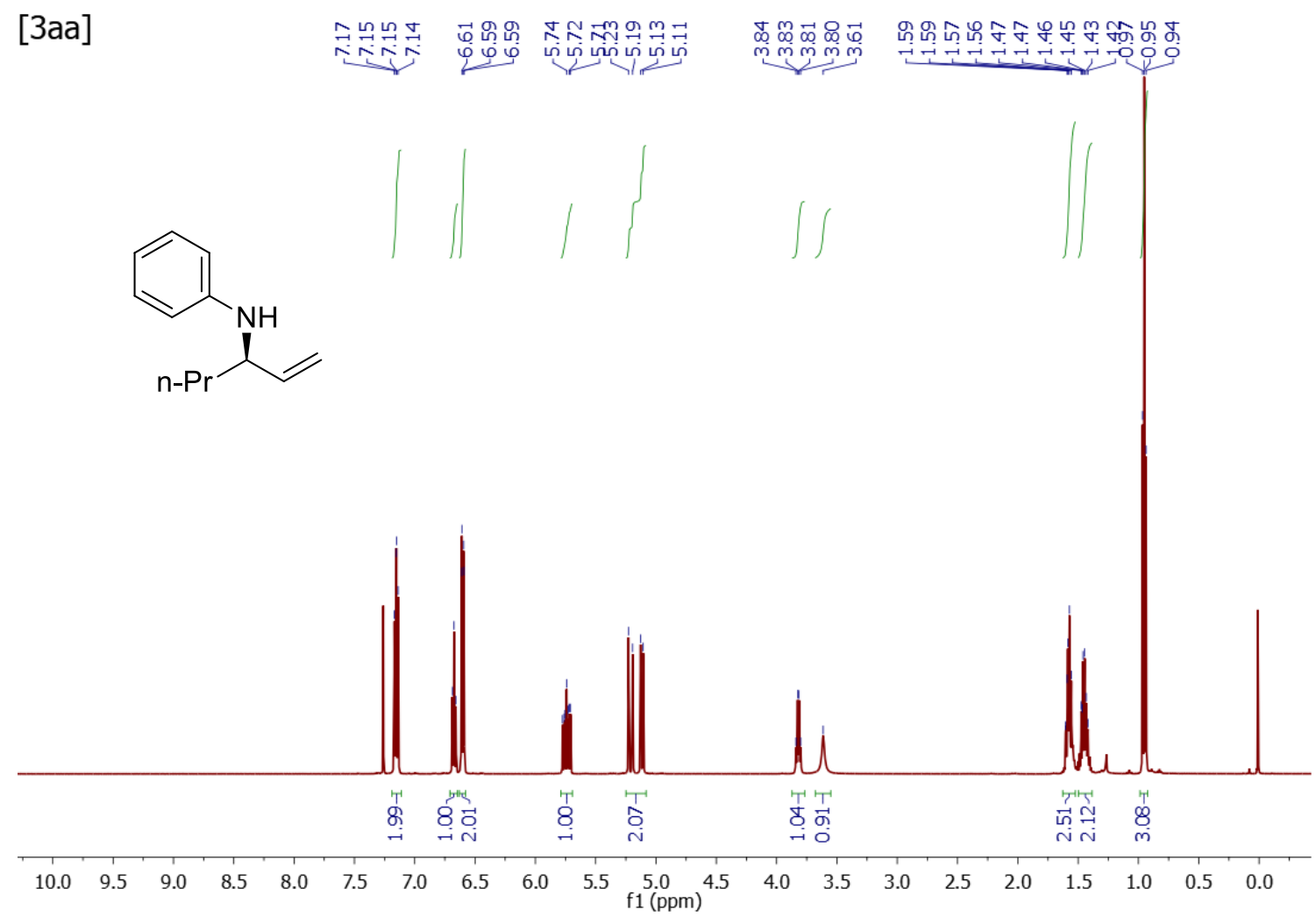

[3aa]
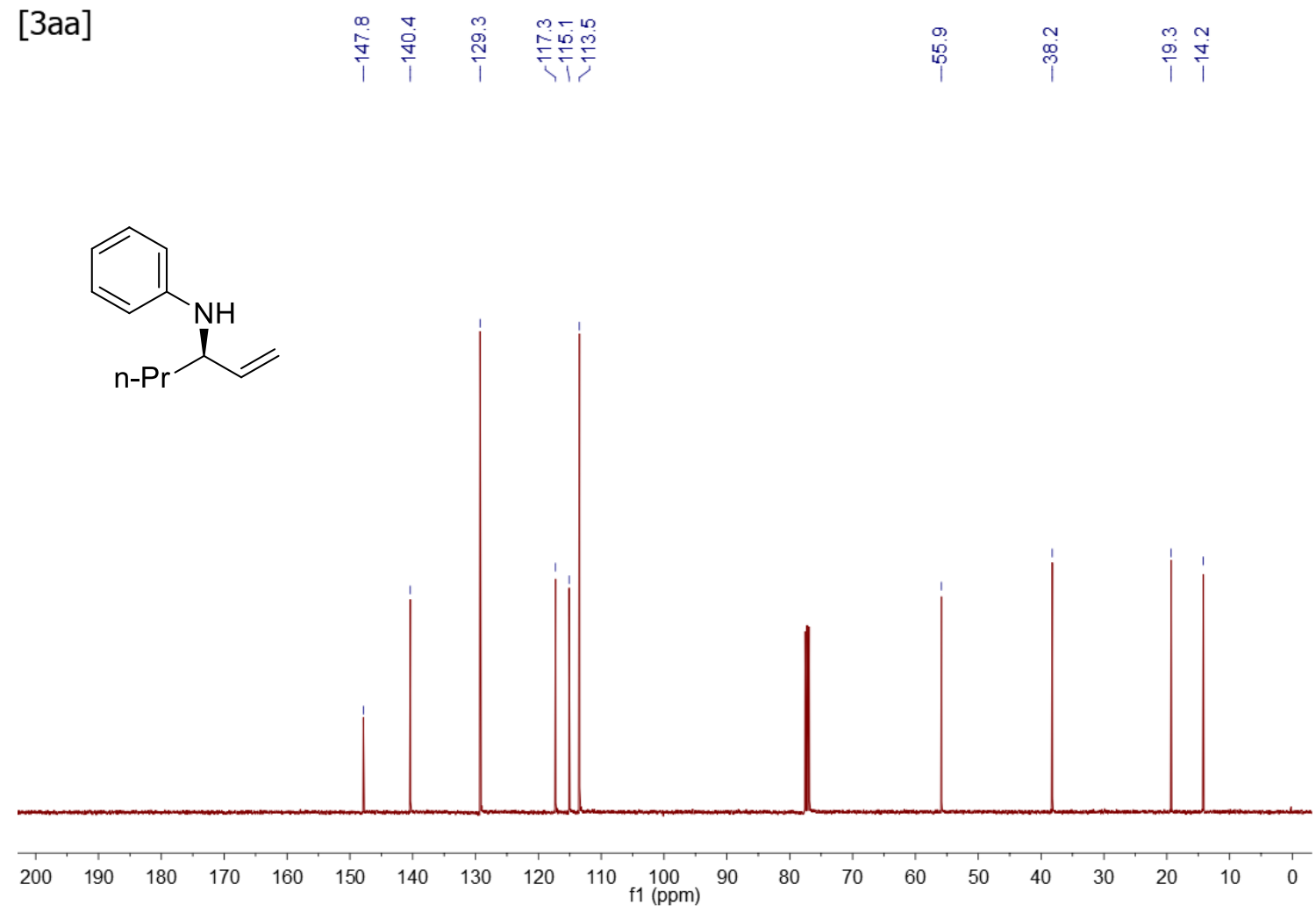

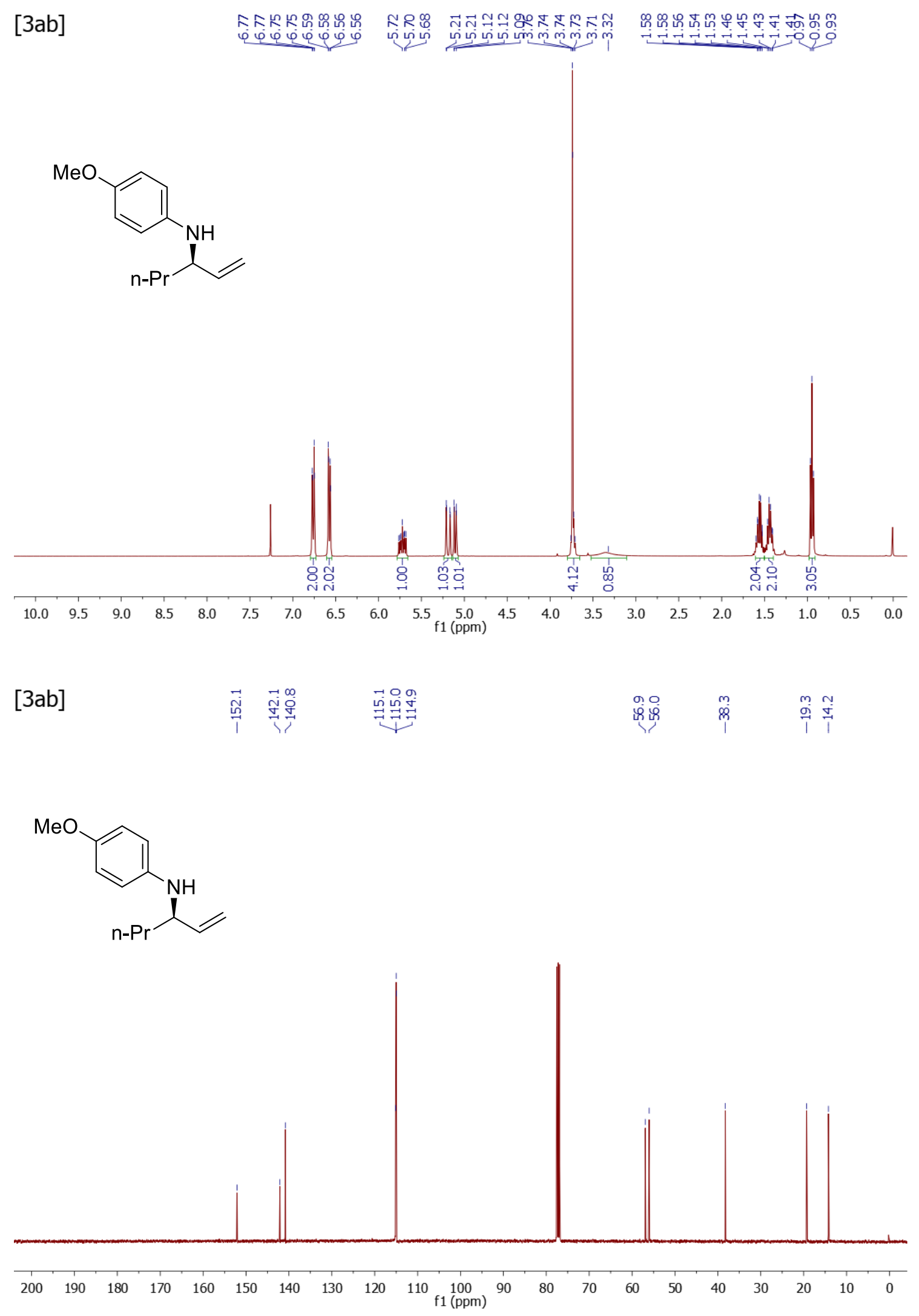


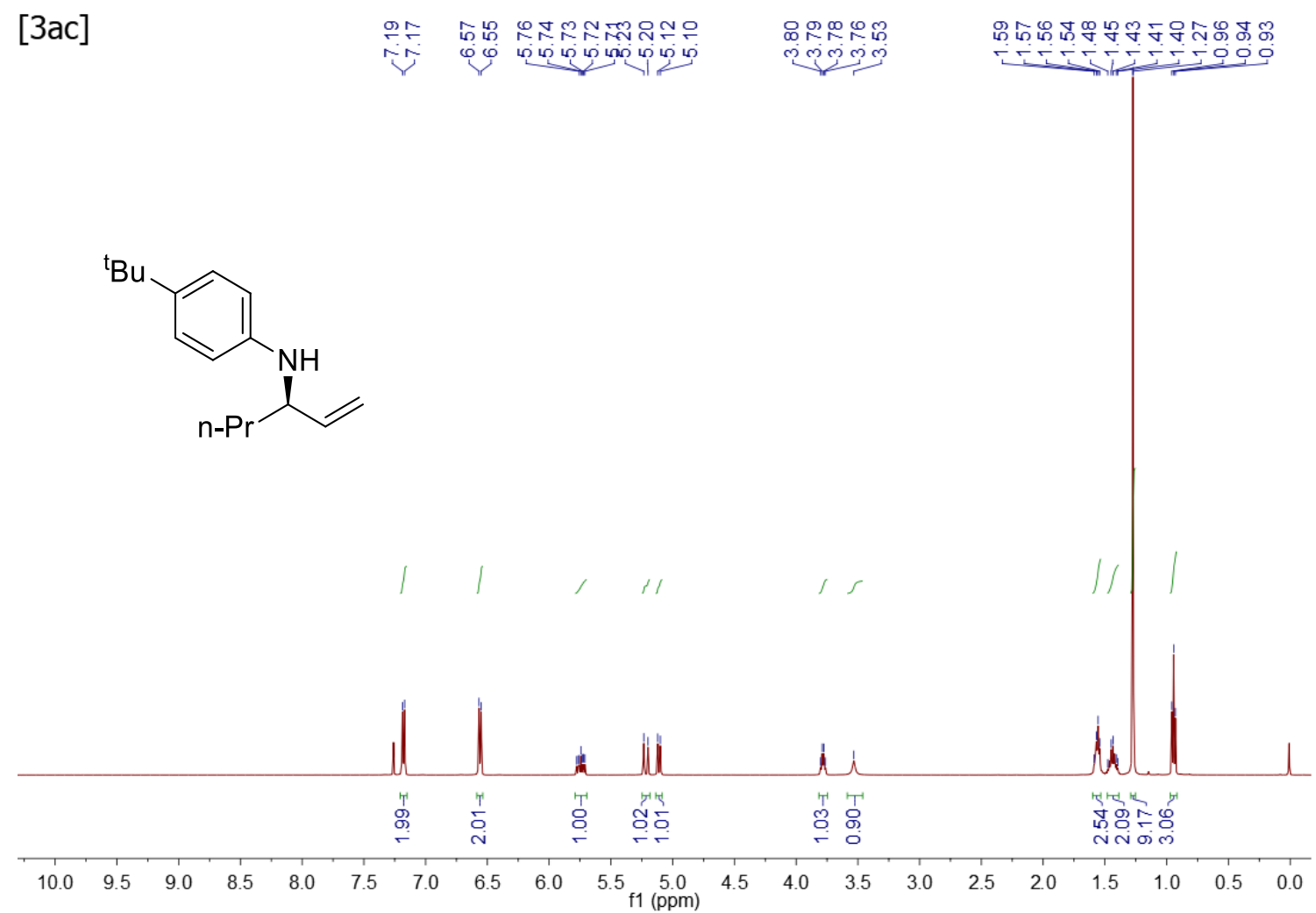

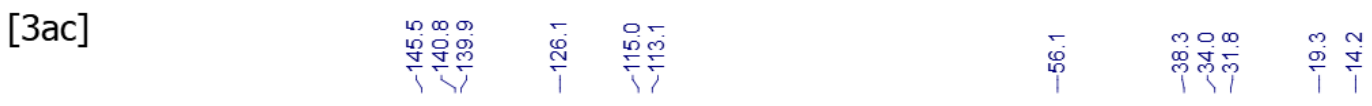

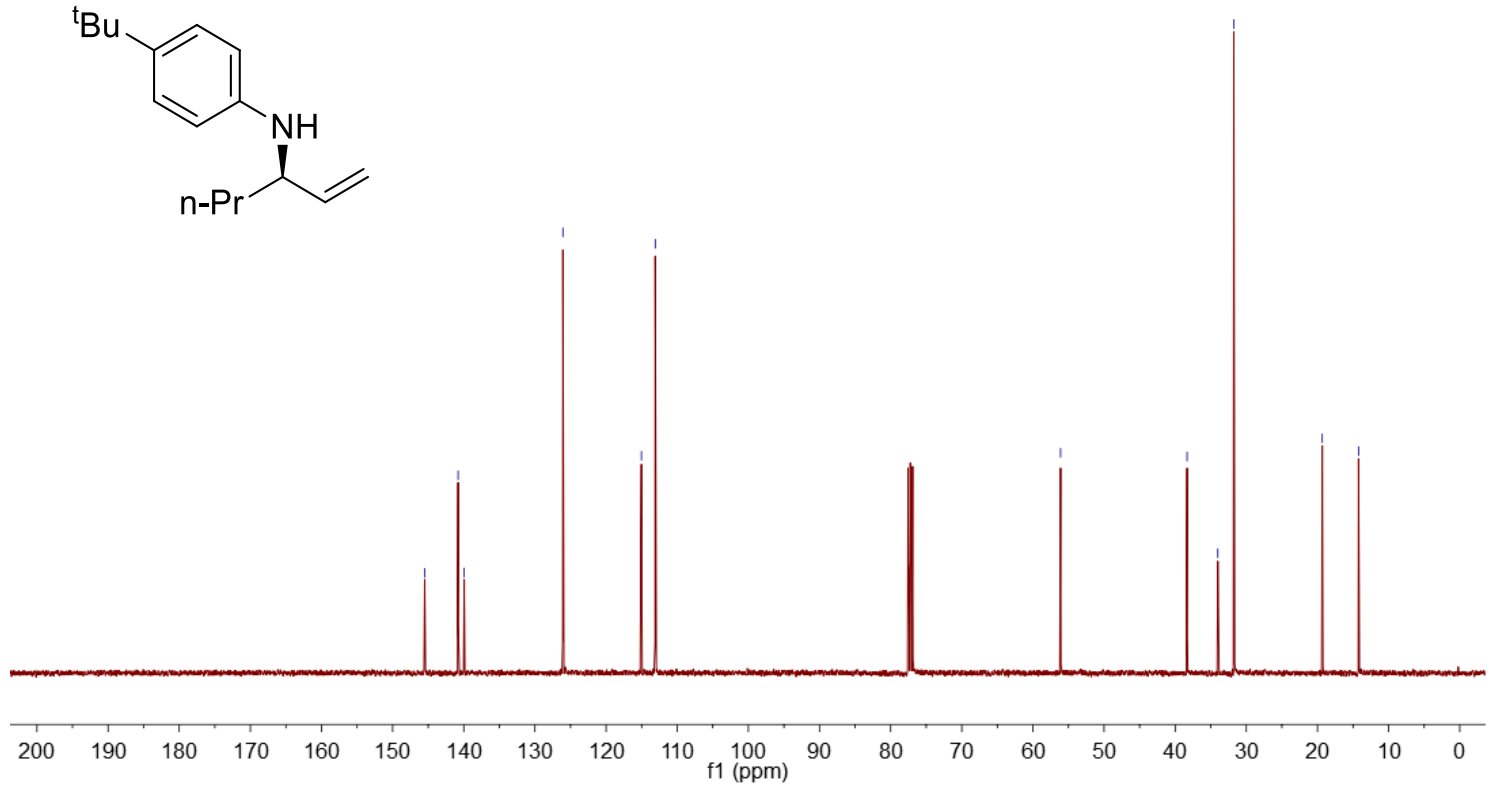


[3ad]<smiles>C=C[C@H](Nc1ccc(Br)cc1)PCC</smiles>

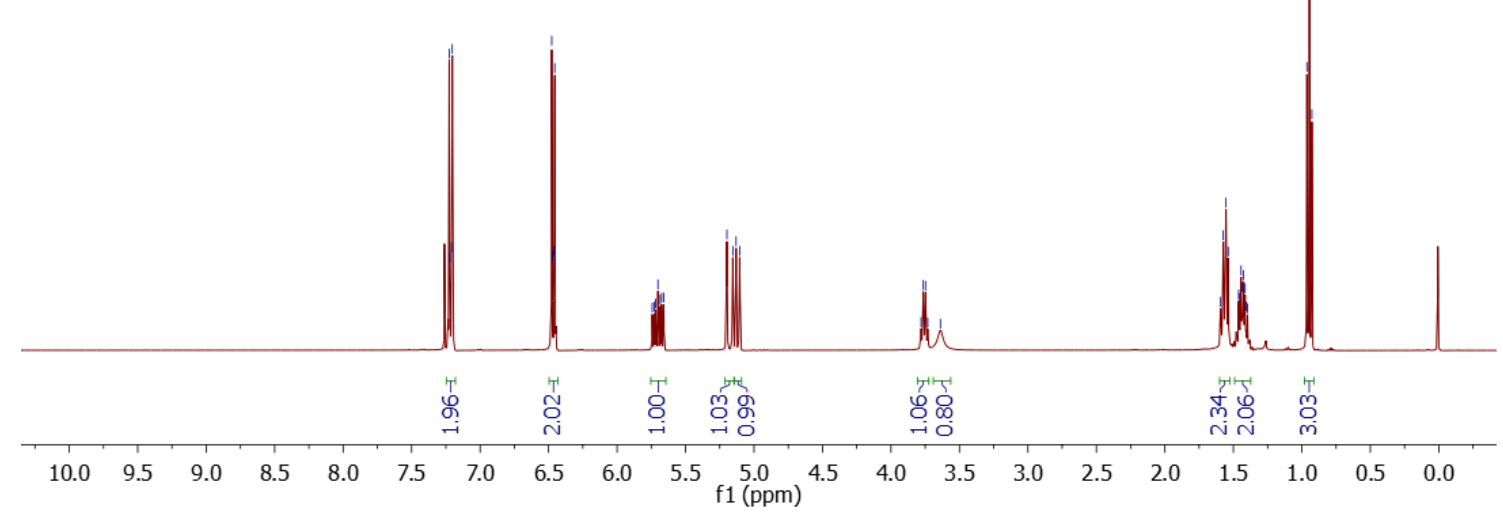

[3ad]

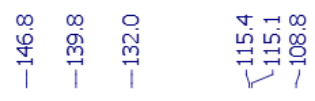
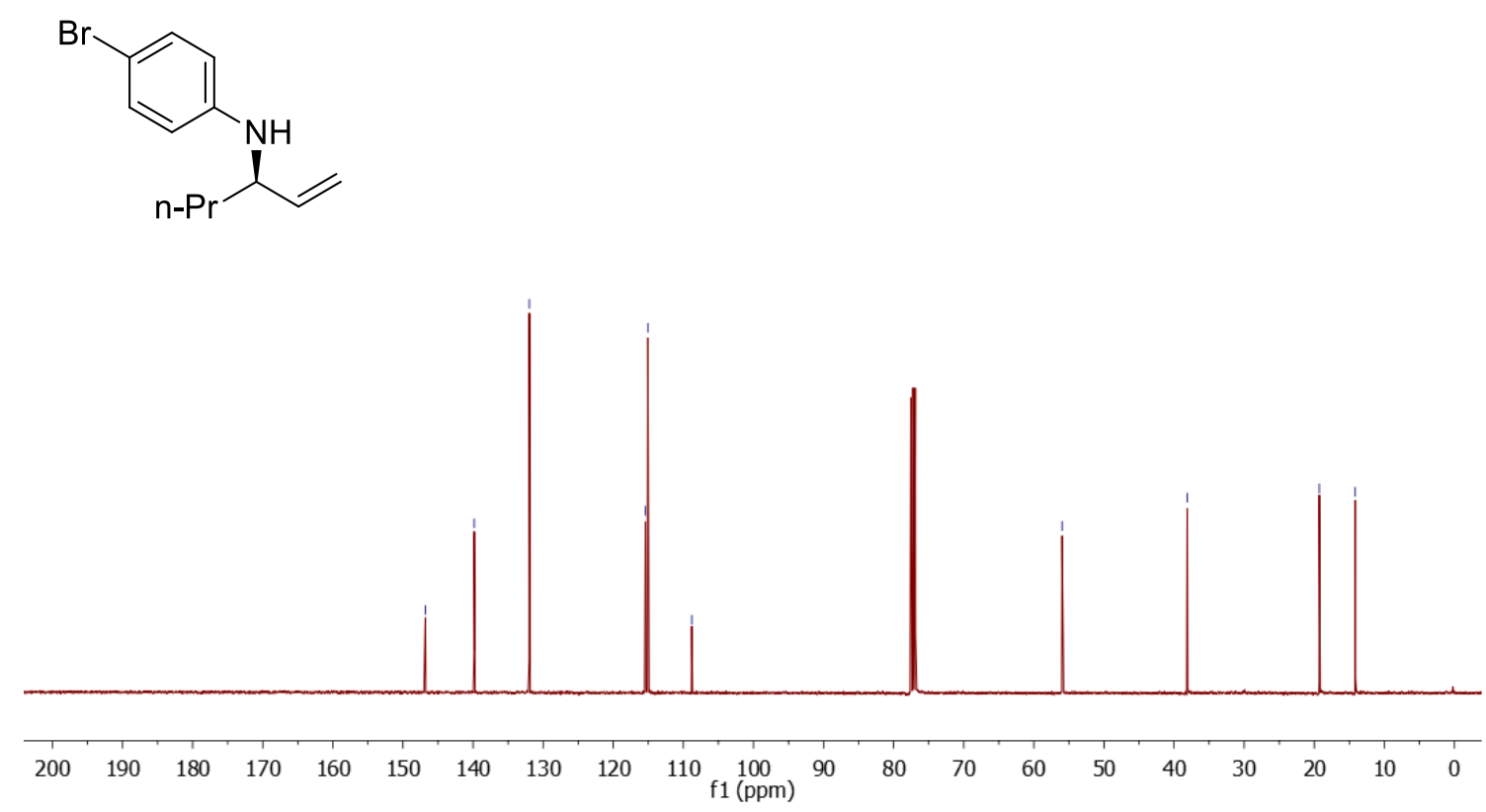

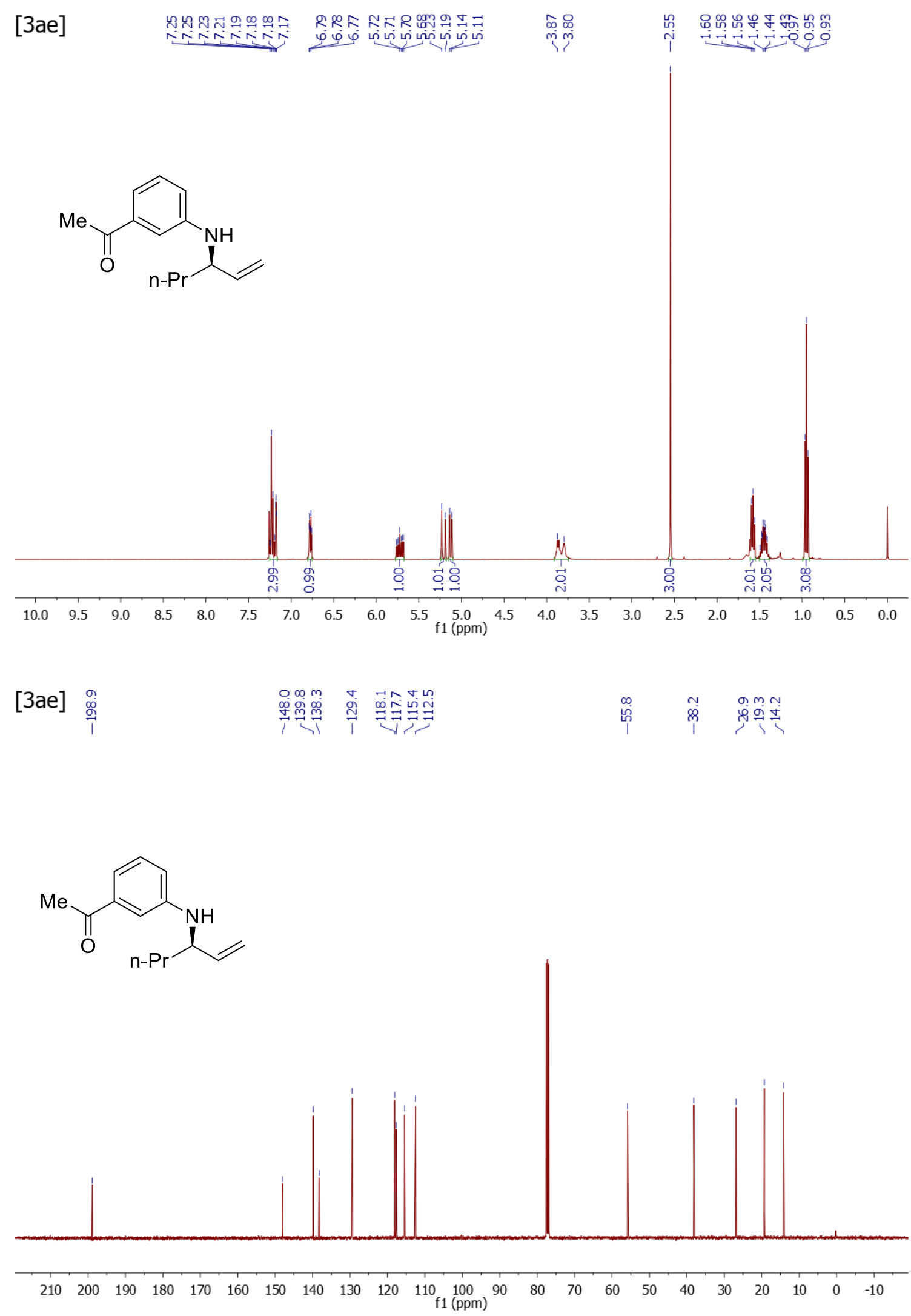

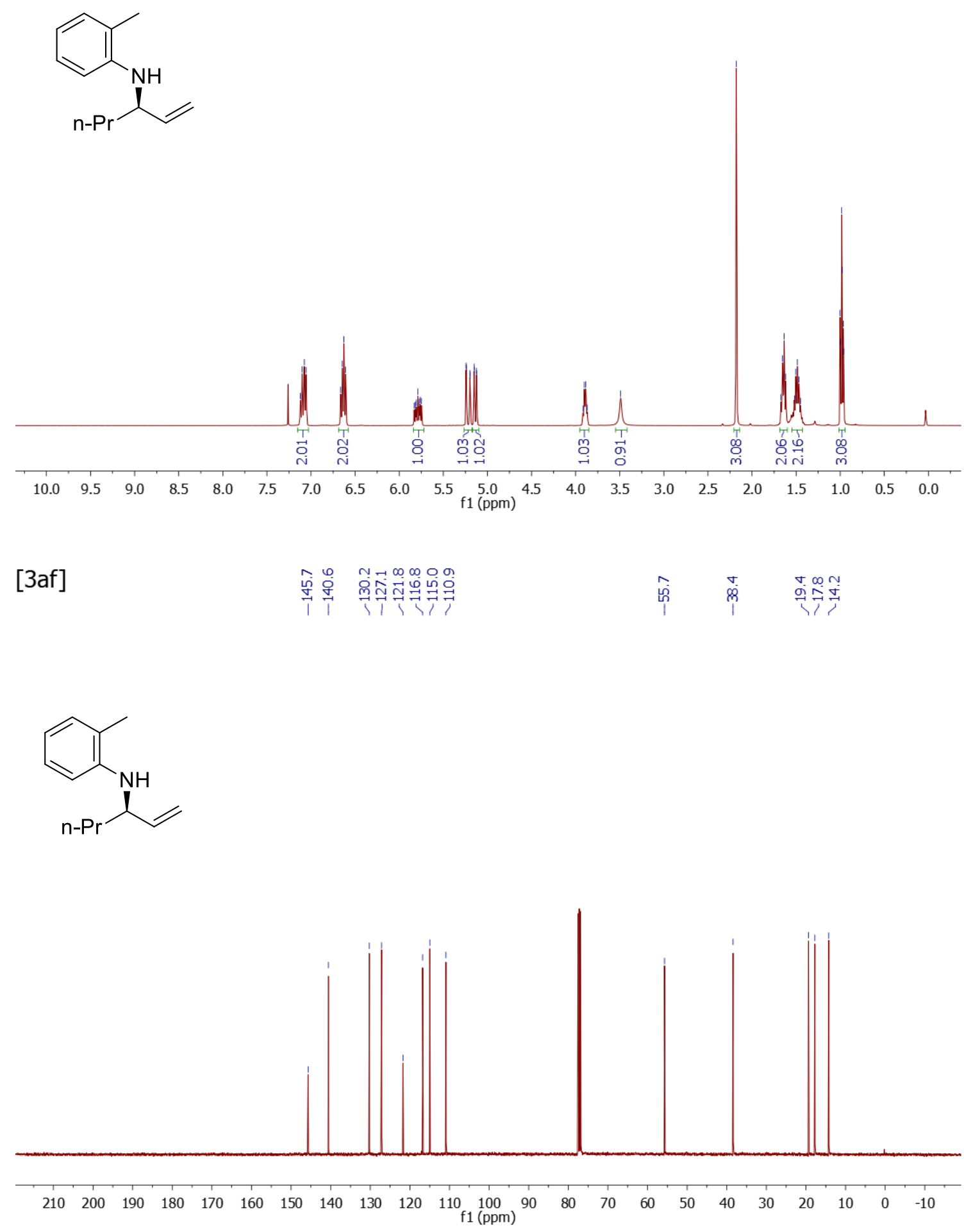
[3ag]

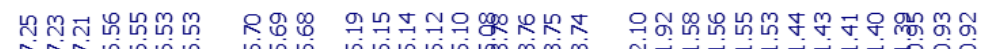

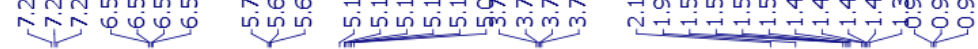
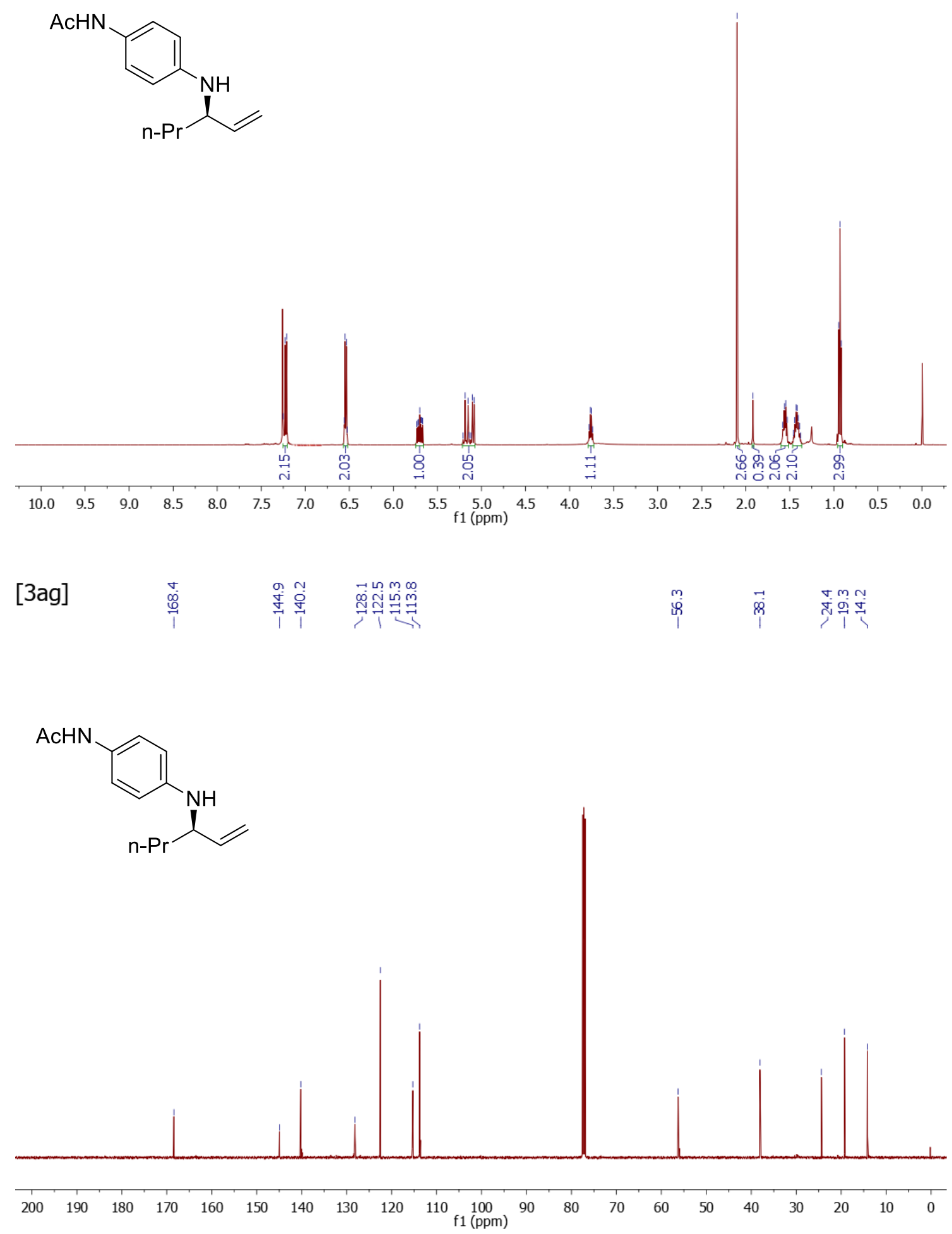

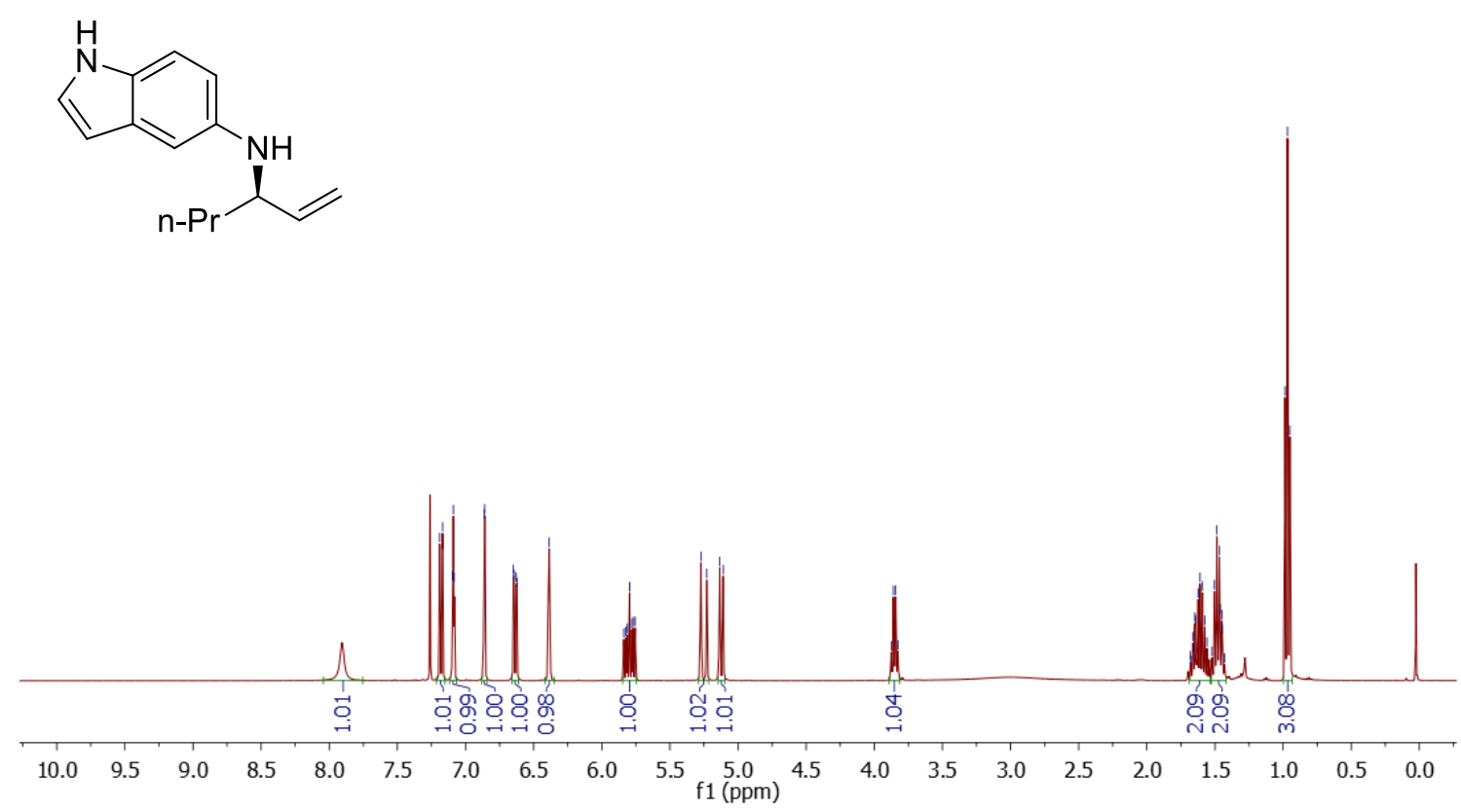

[3ah]

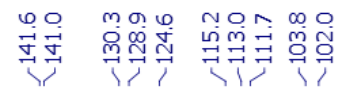

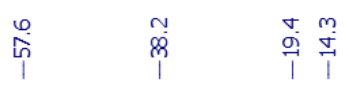<smiles>C=C[C@H](Nc1ccc2[nH]ccc2c1)PCC</smiles>

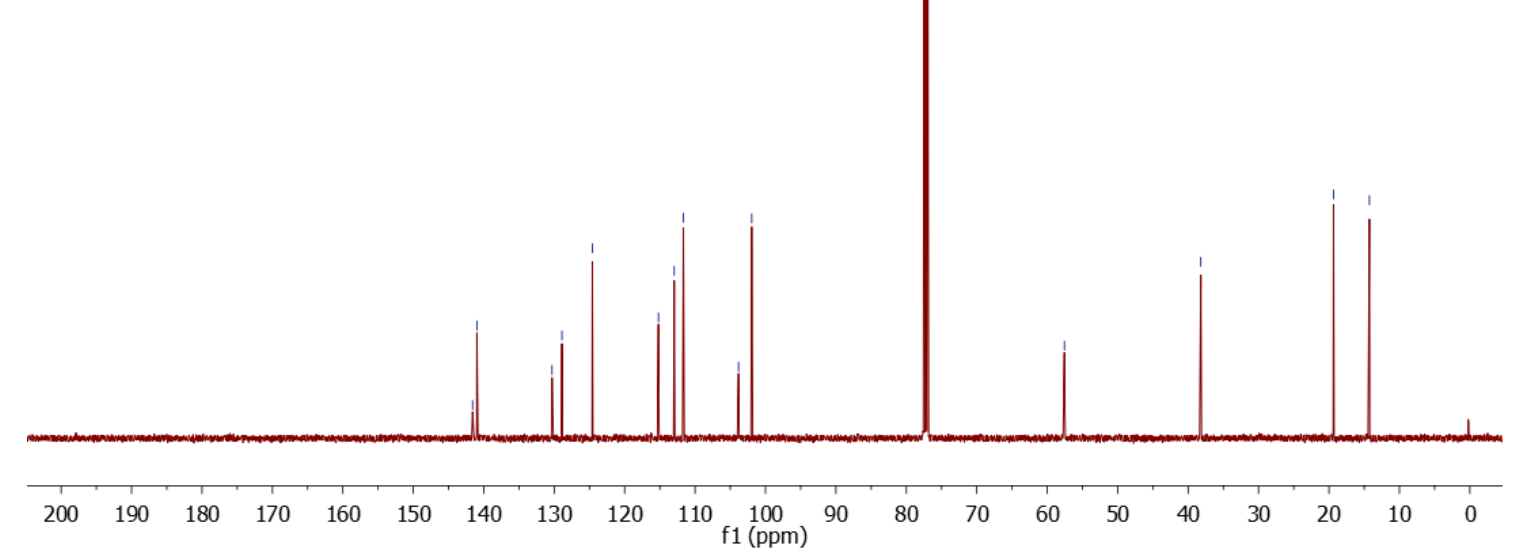


[3ai]<smiles>C=CC(CCCC)N(C)c1ccccc1</smiles>

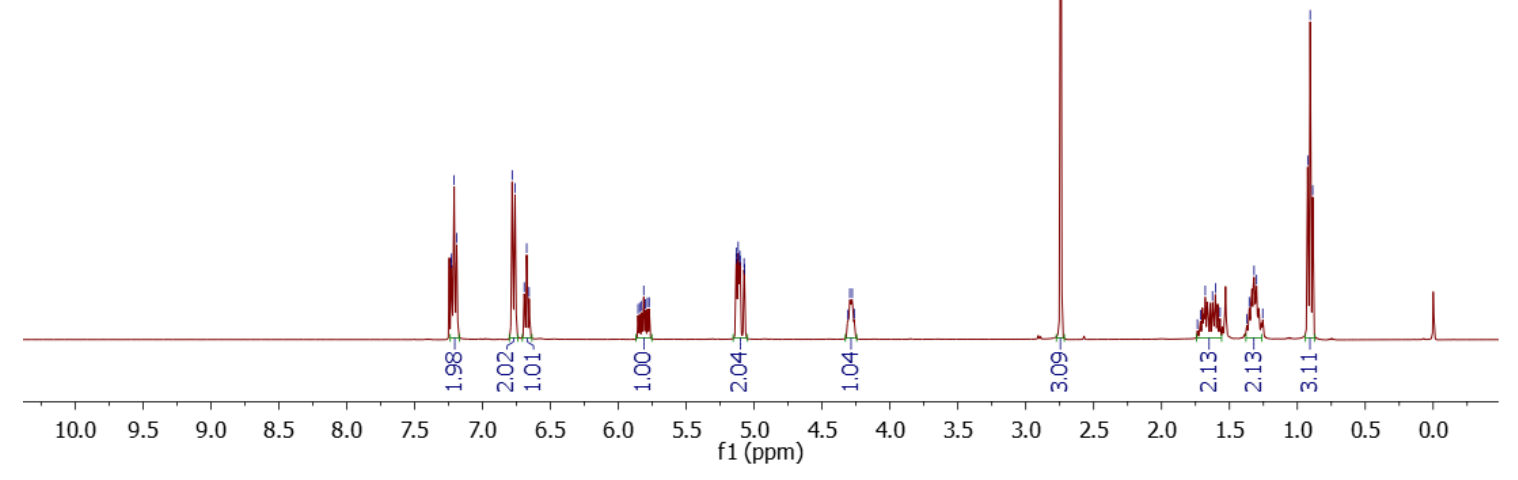

[3ai]<smiles>C=CC(CCC)N(C)c1ccccc1</smiles>

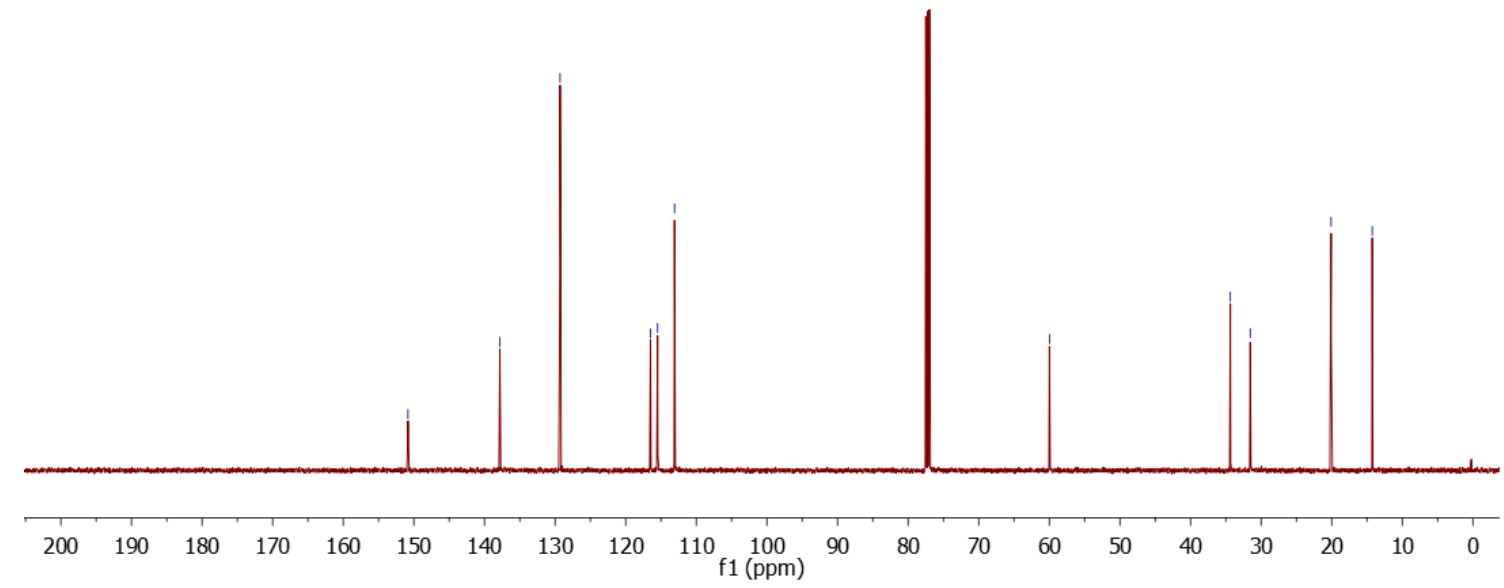


[3aj]
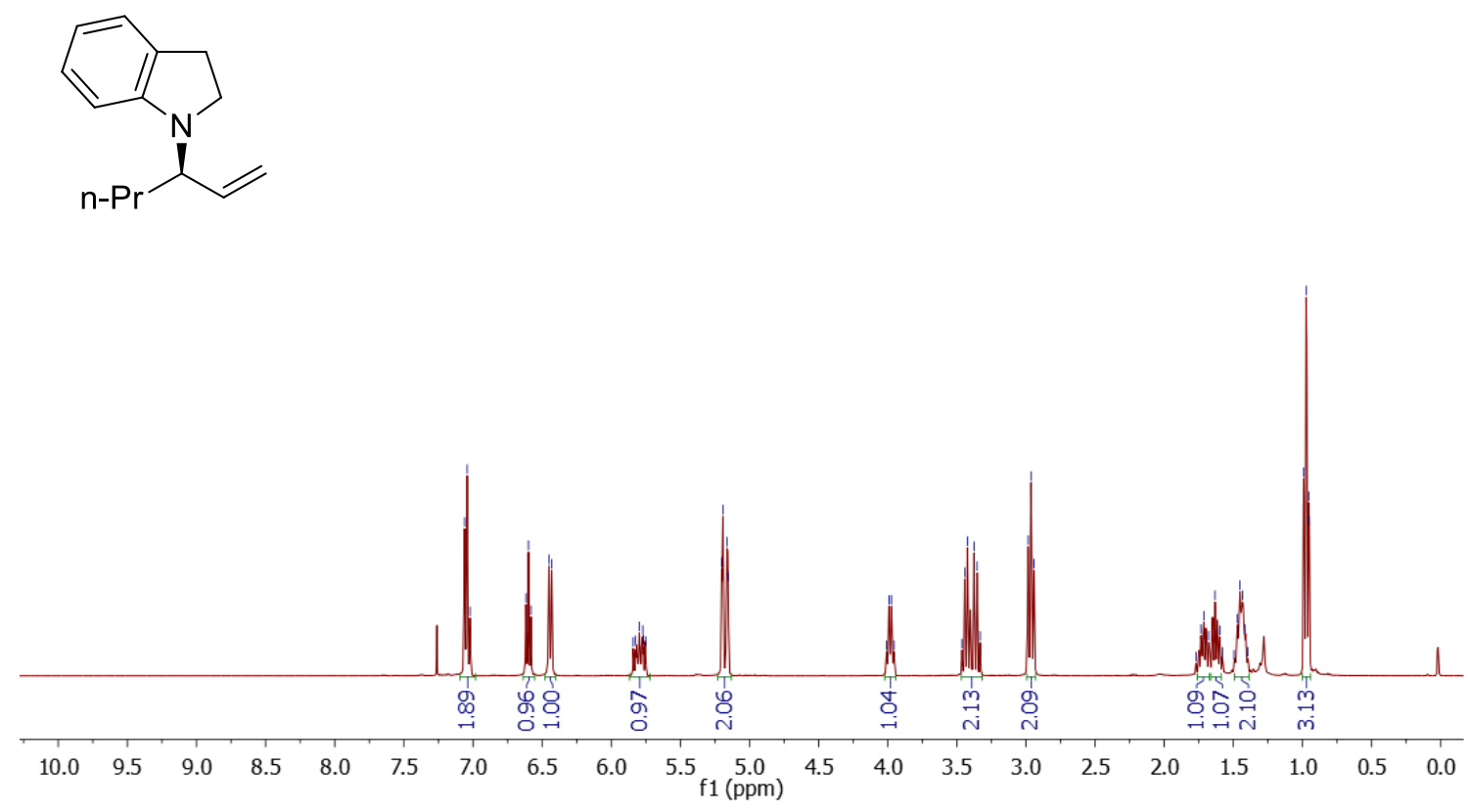

[3aj]<smiles>C=CC(C(=C)C)N1CCc2ccccc21</smiles>

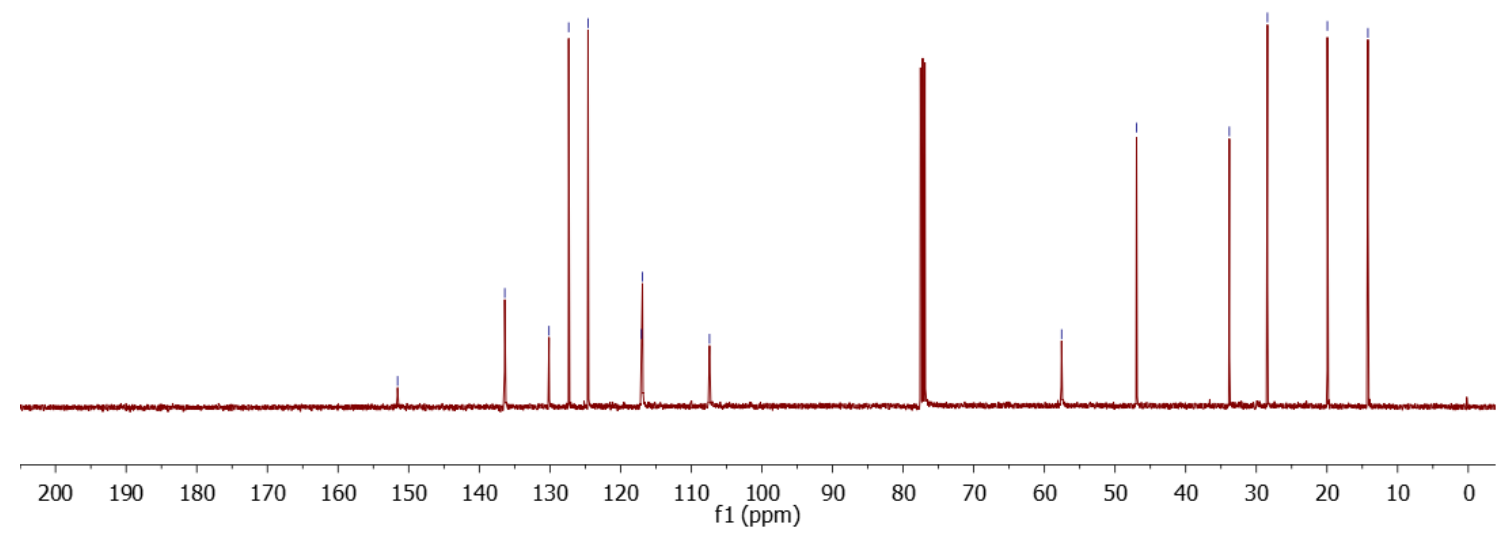




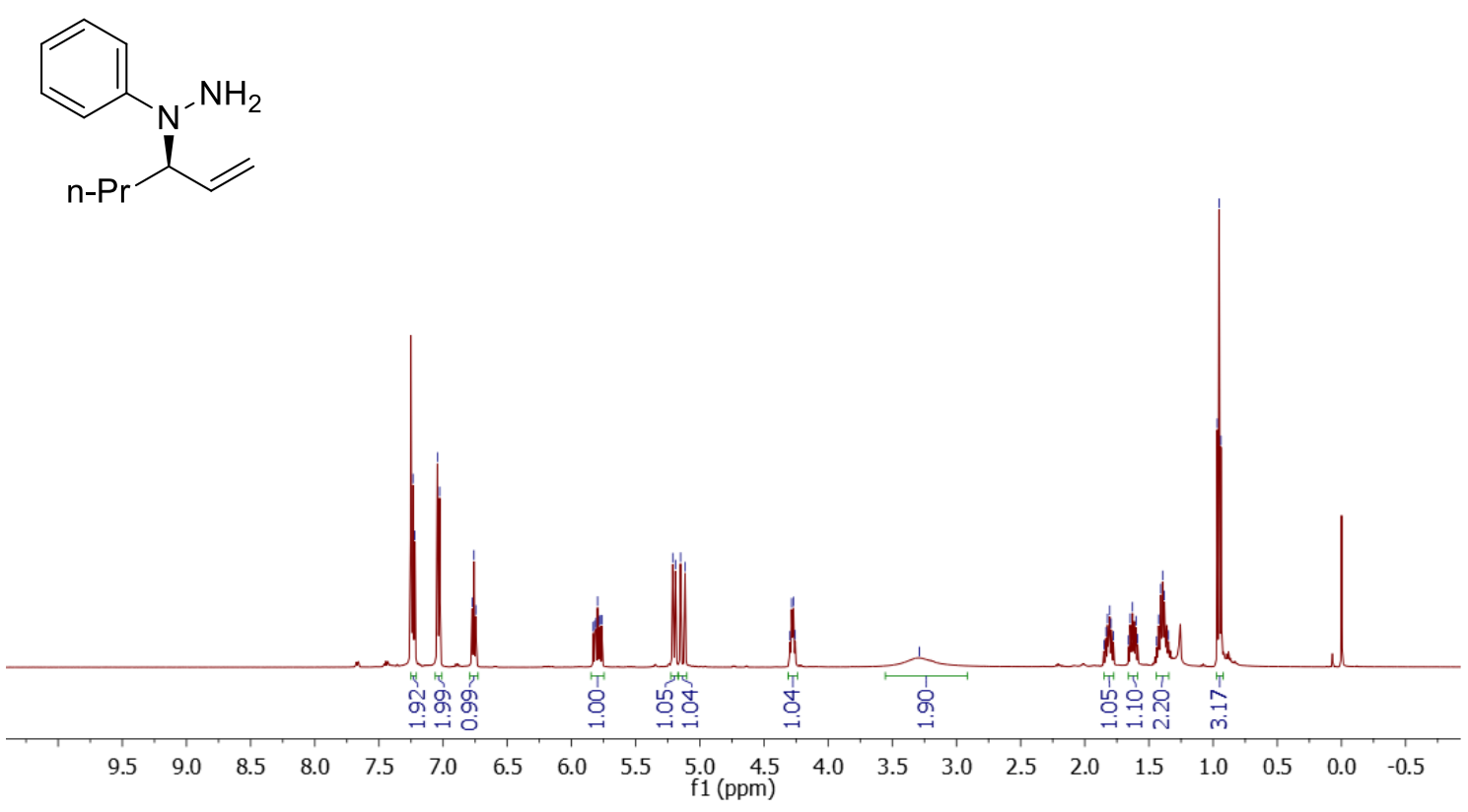

[3ak]<smiles>C=CC(CCCC)N(N)c1ccccc1</smiles>

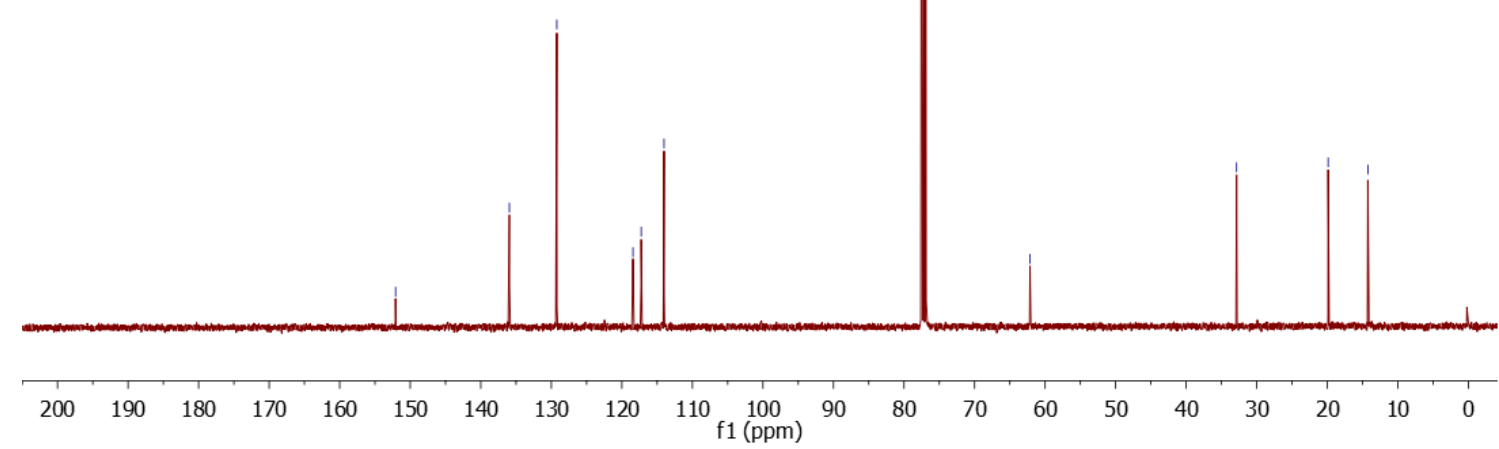


[3bb]

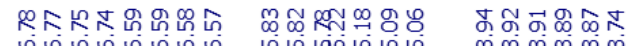

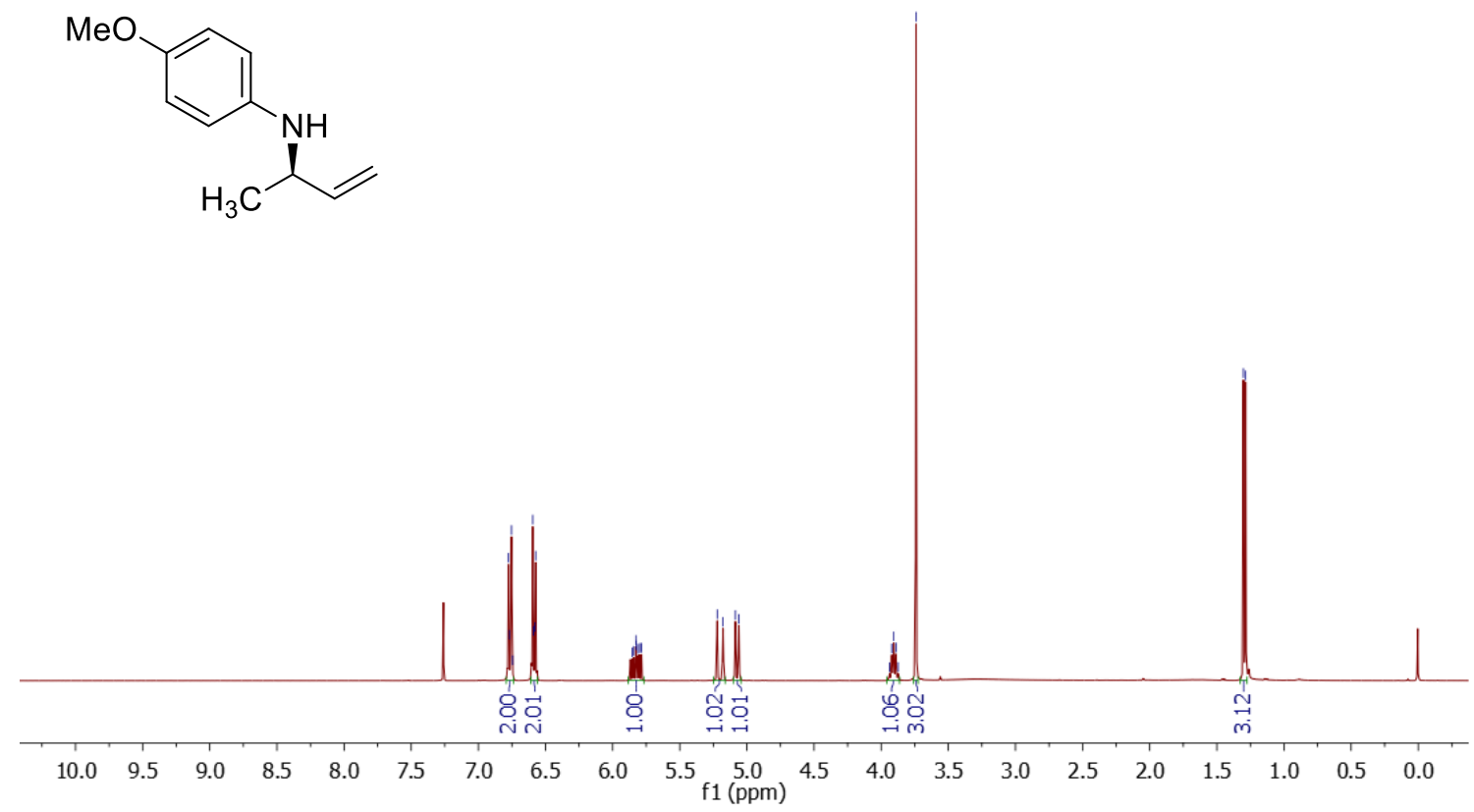

[3bb]

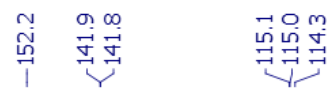

ำ ำ

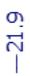<smiles>C=CC(C)Nc1ccc(OC)cc1</smiles>

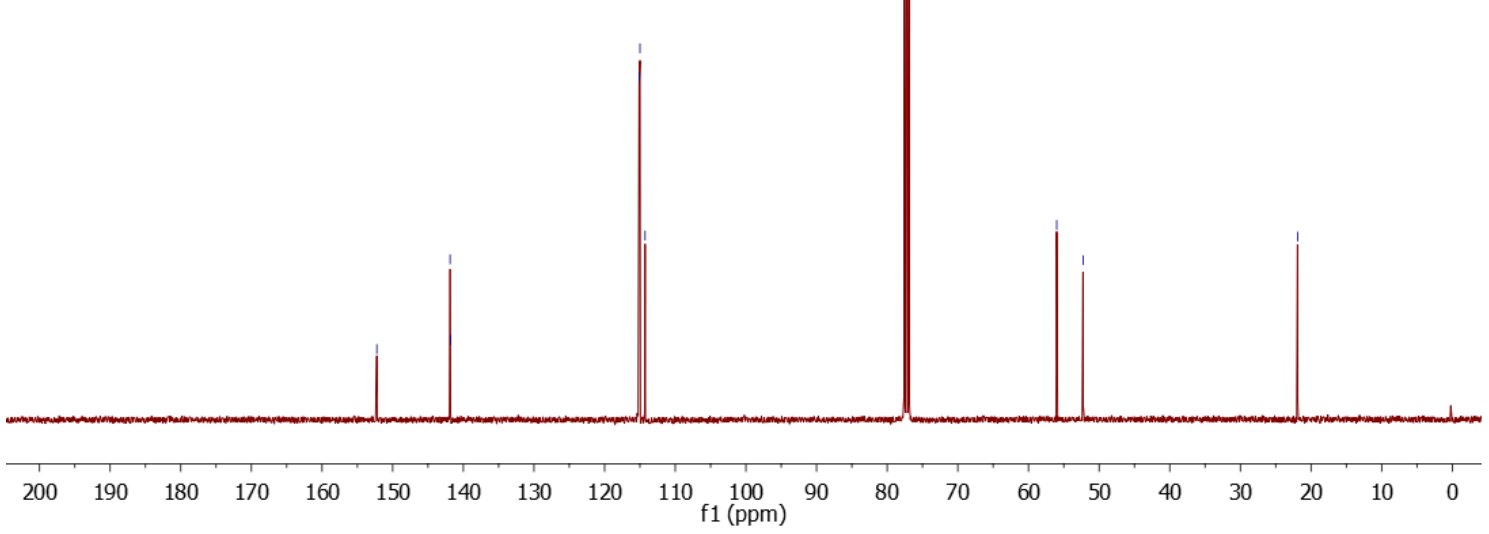




\section{[3cb]}

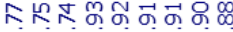<smiles>C=CC(CCc1ccccc1)Nc1ccc(OC)cc1</smiles>

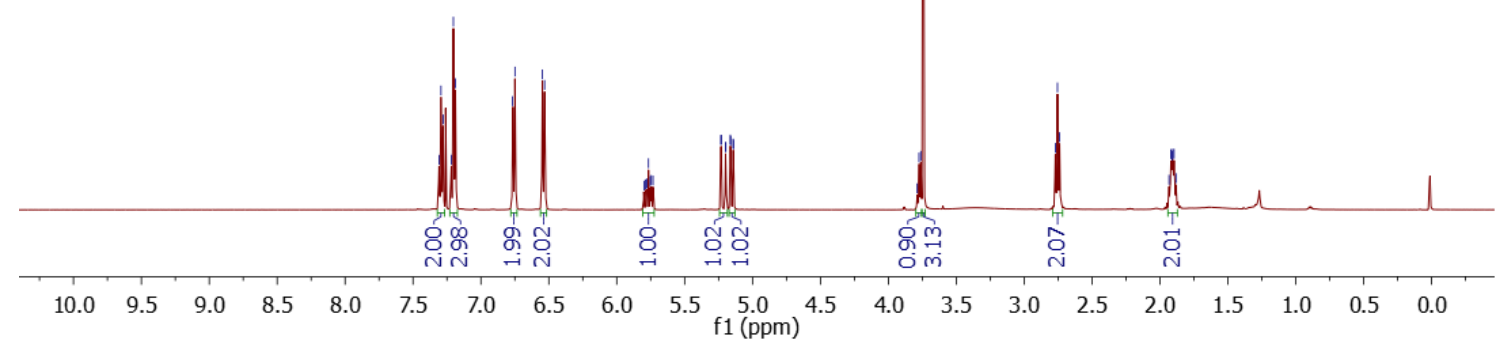

[3cb]

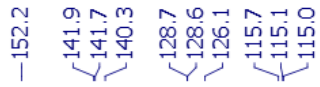
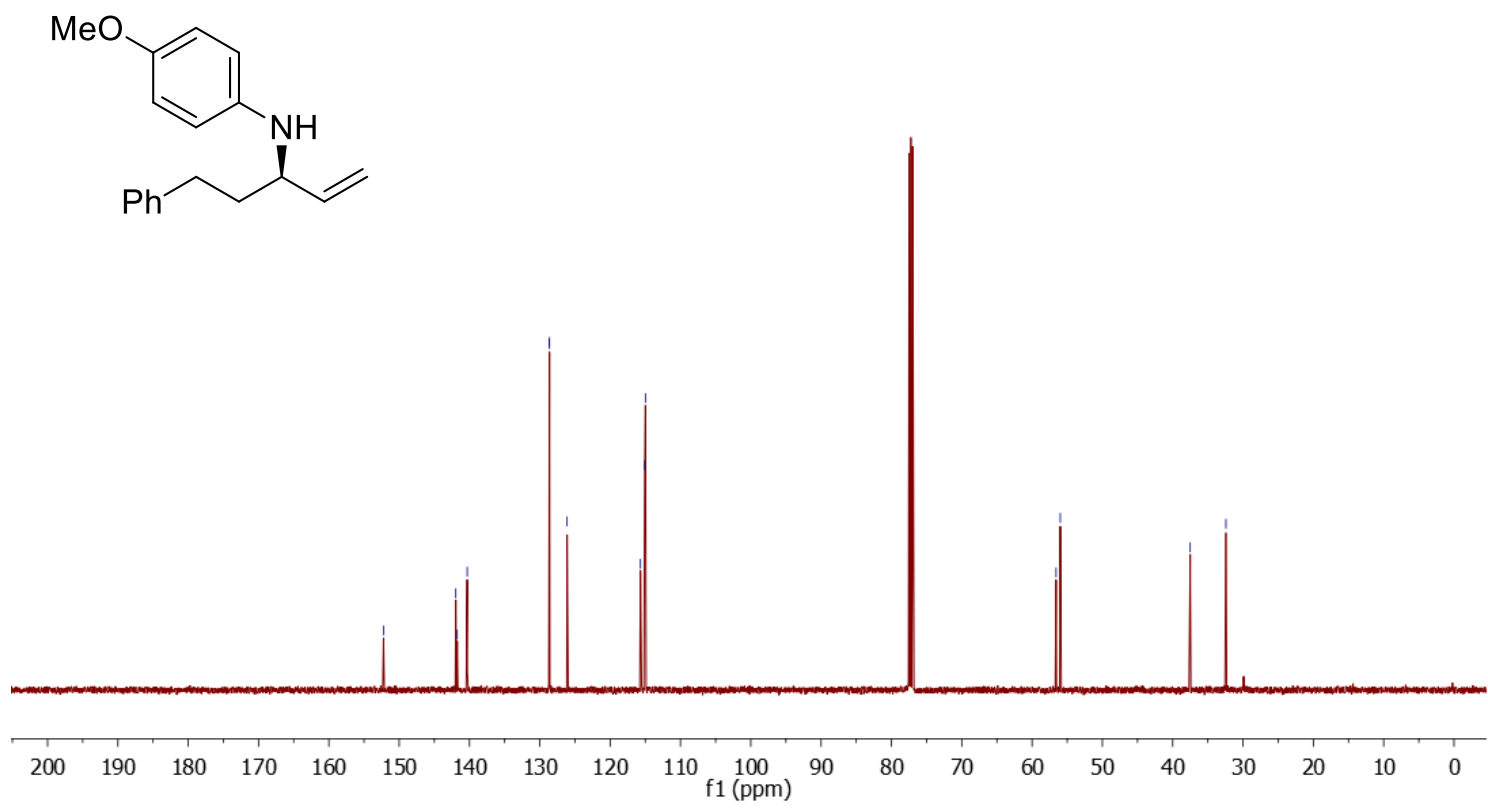


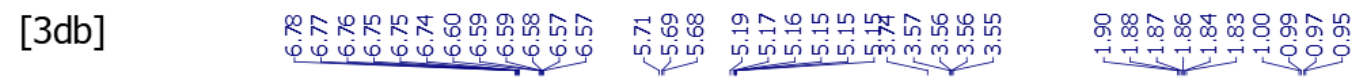

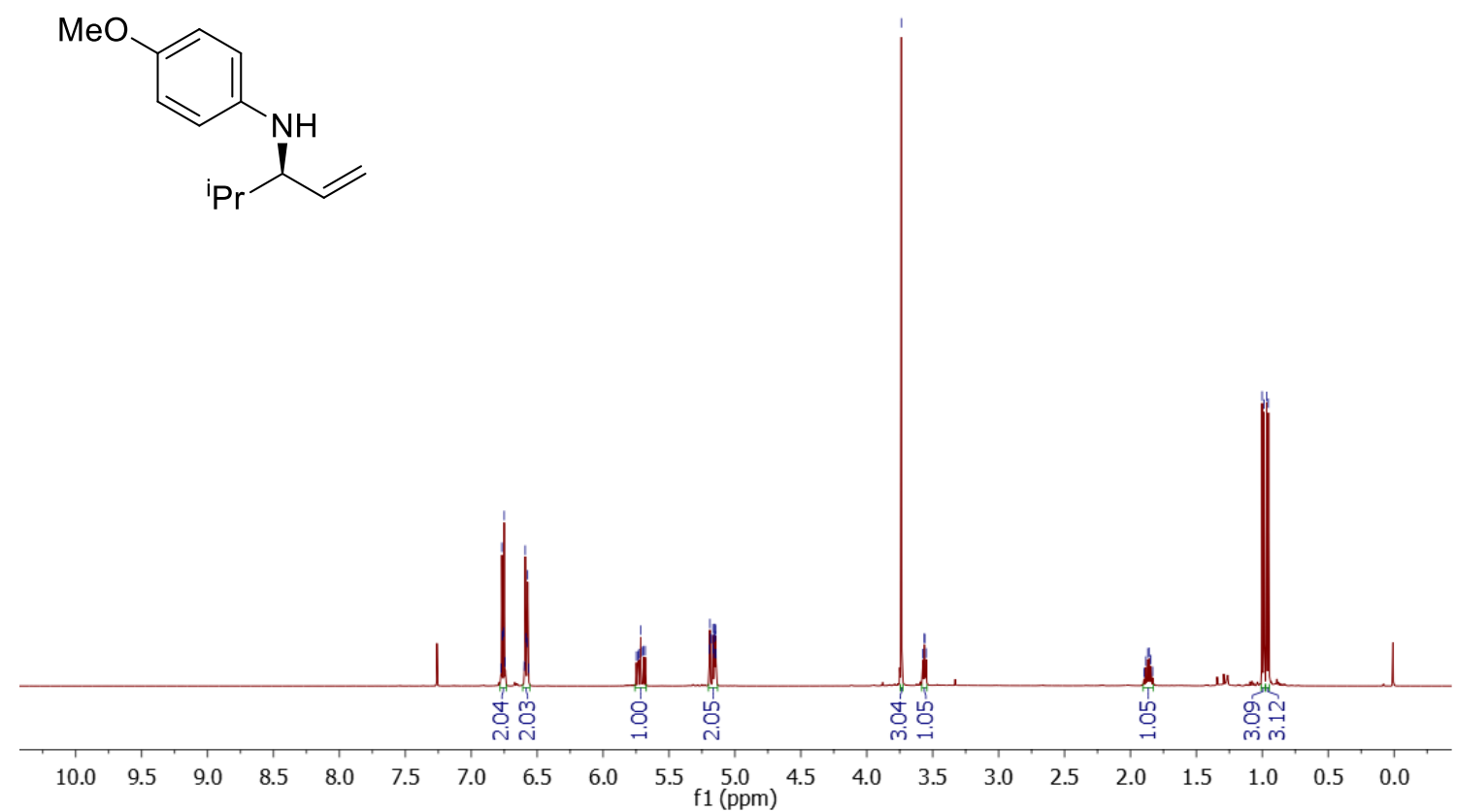

[3db]

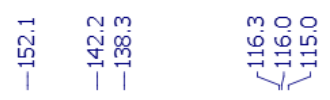

$\begin{array}{ll}\infty & 0 \\ \stackrel{1}{0} & \stackrel{1}{1}\end{array}$

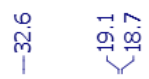

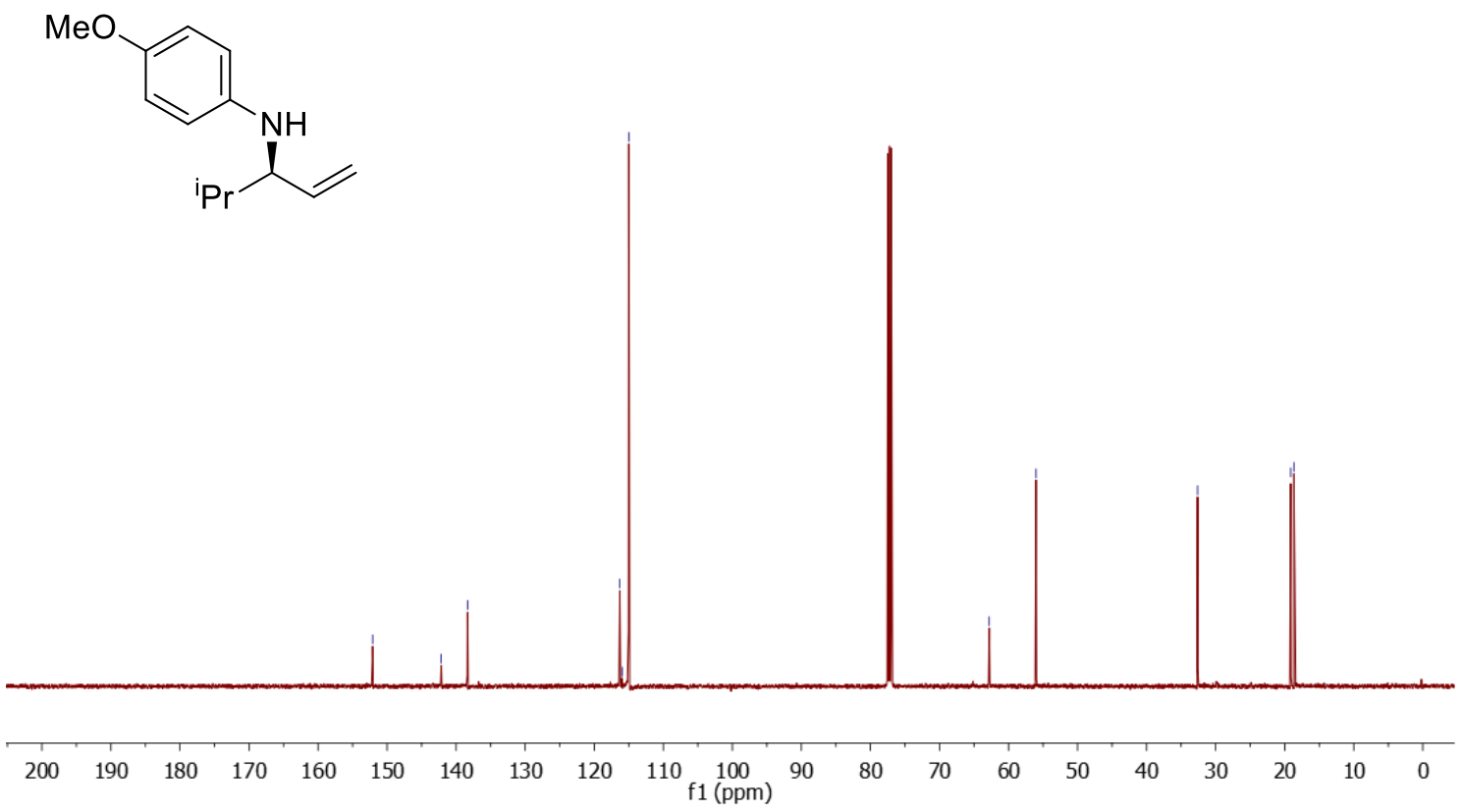




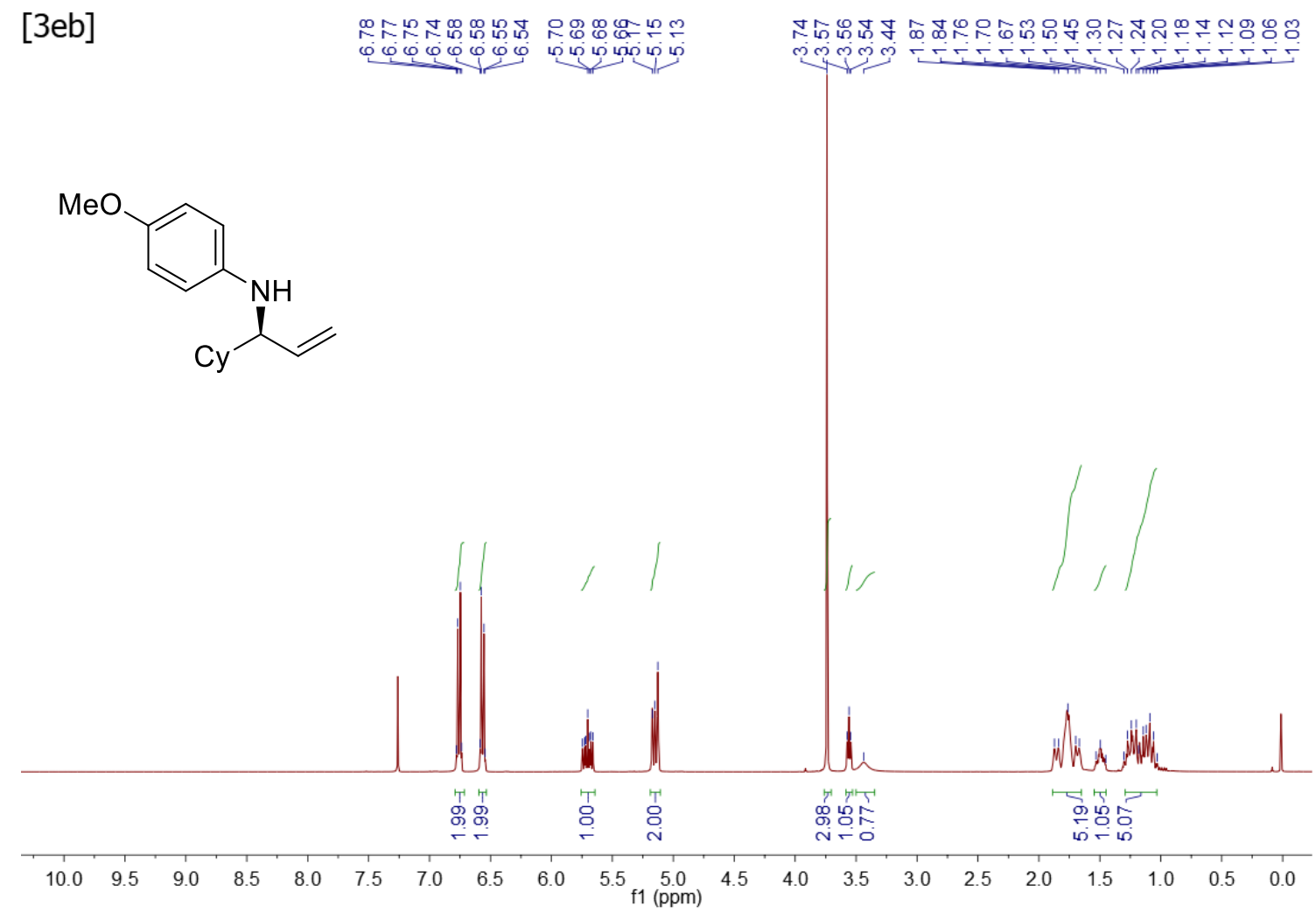

[3eb]

\begin{tabular}{|c|c|c|}
\hline 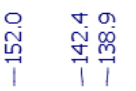 & 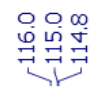 & 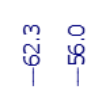 \\
\hline
\end{tabular}<smiles>C=C[C](Nc1ccc(OC)cc1)C1CCCCC1</smiles>

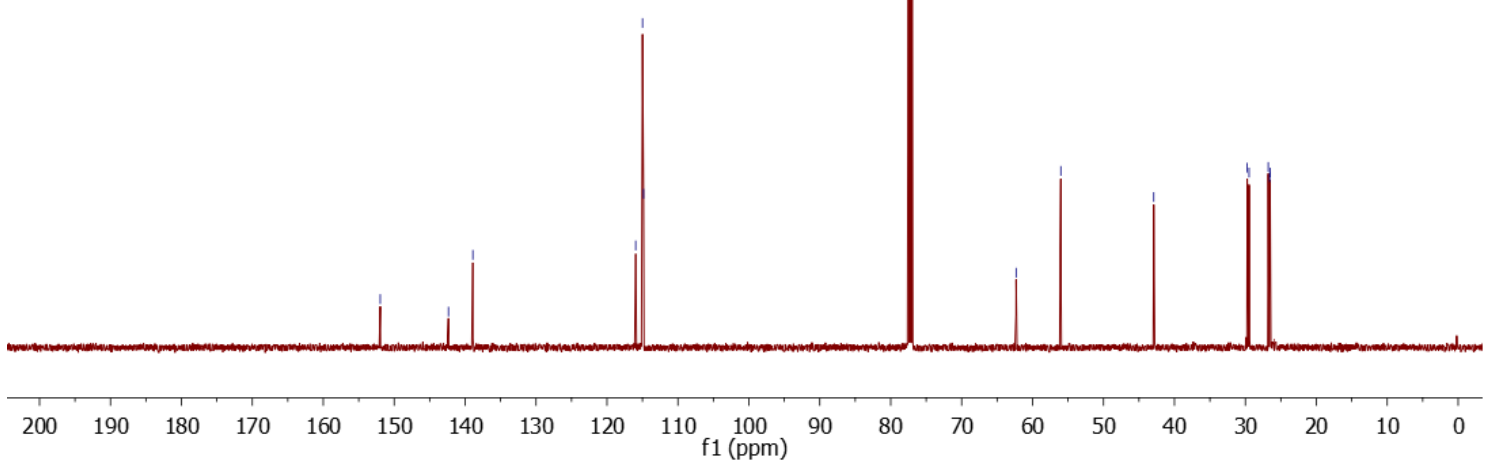


$[3 \mathrm{fb}]$
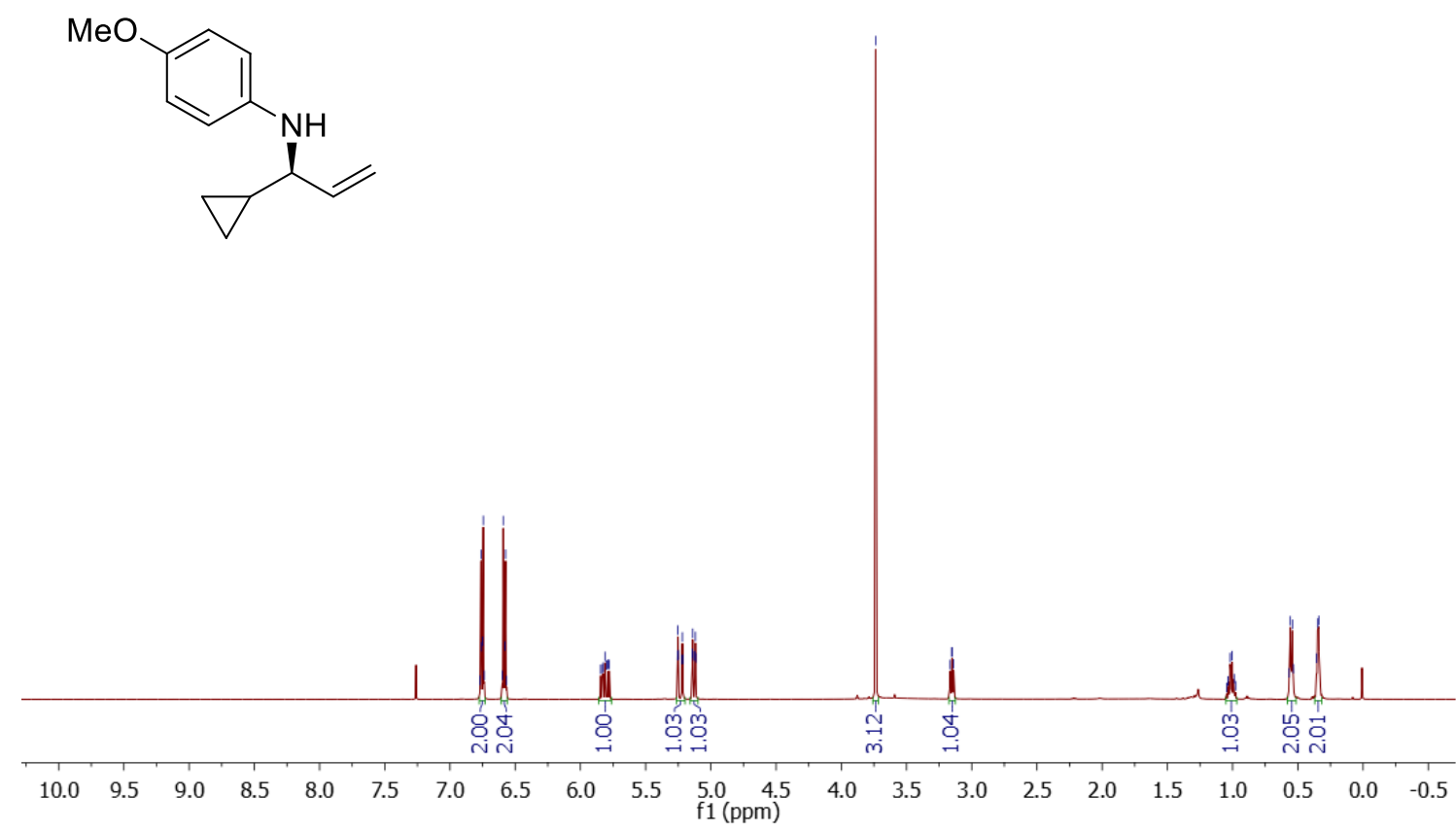

[3fb]

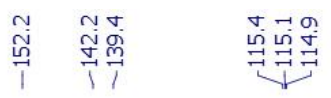

๓ก

$\stackrel{\circ}{\stackrel{n}{1}}$<smiles>C=C[C@@H](Nc1ccc(OC)cc1)C1CC1</smiles>

200

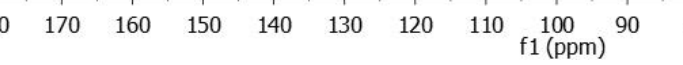


[3gb]

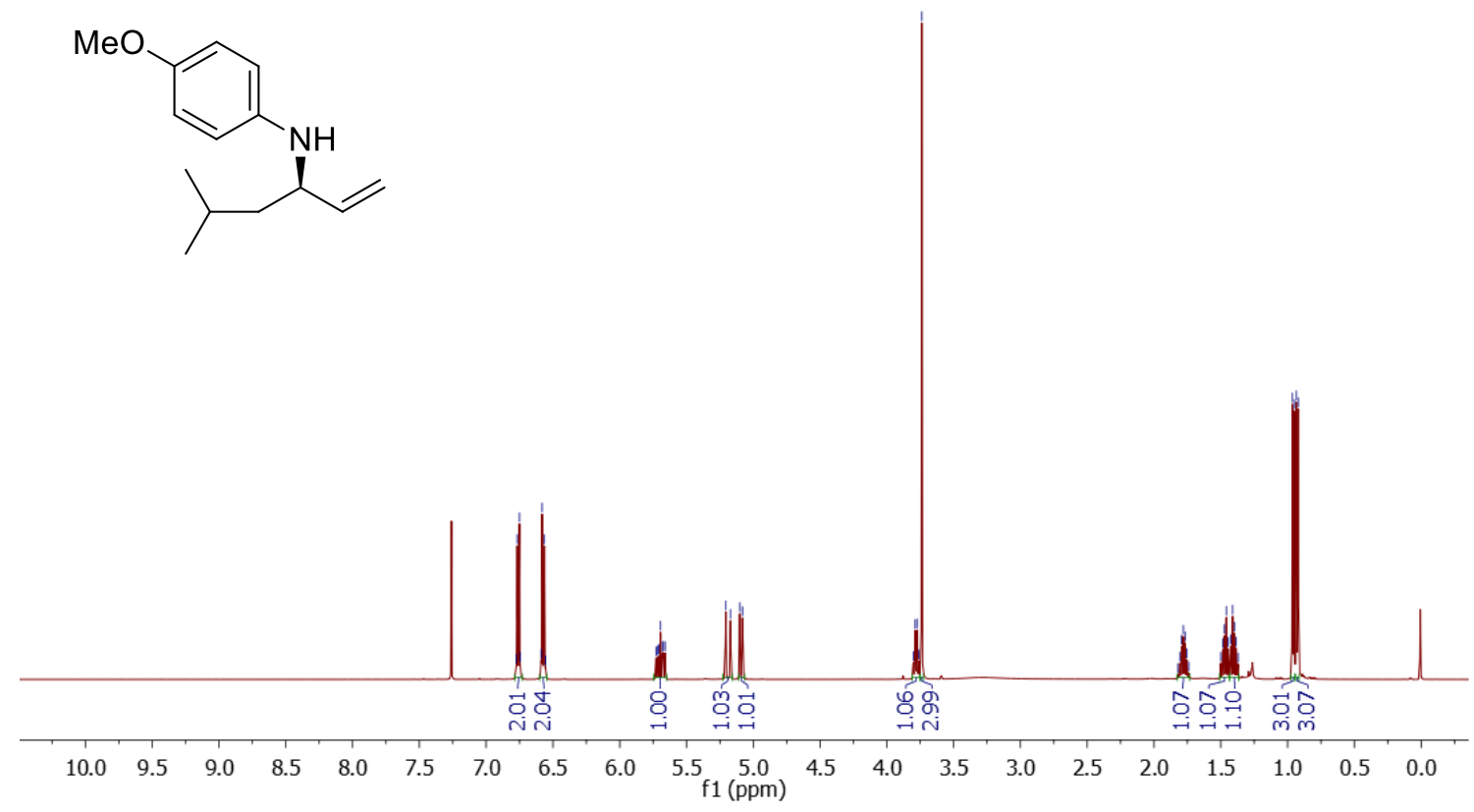

[3gb]

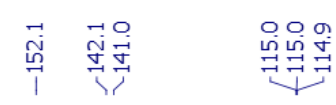

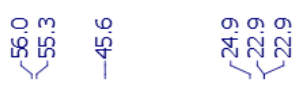

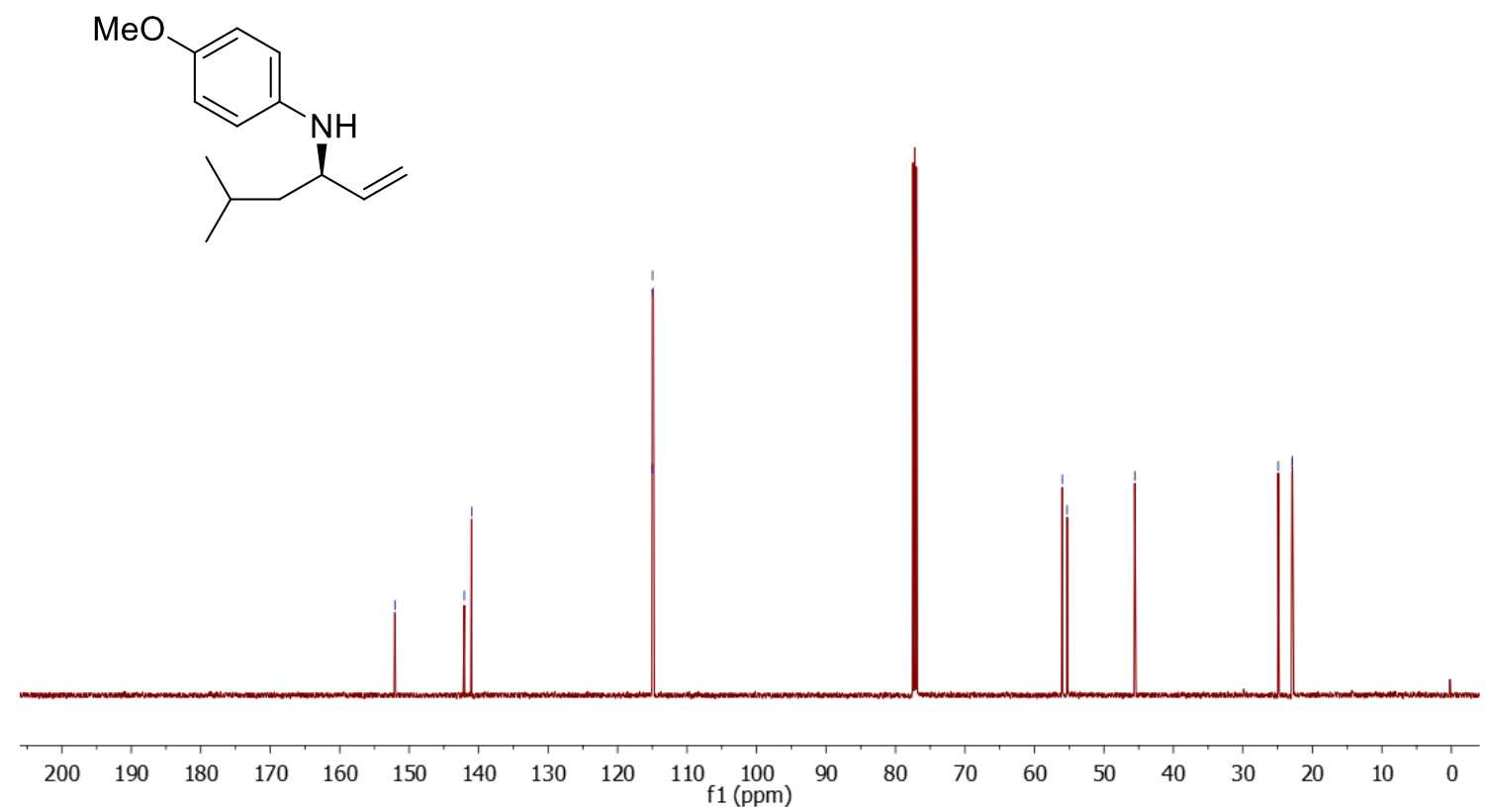




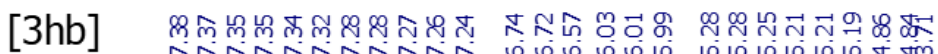

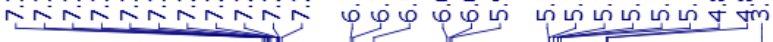

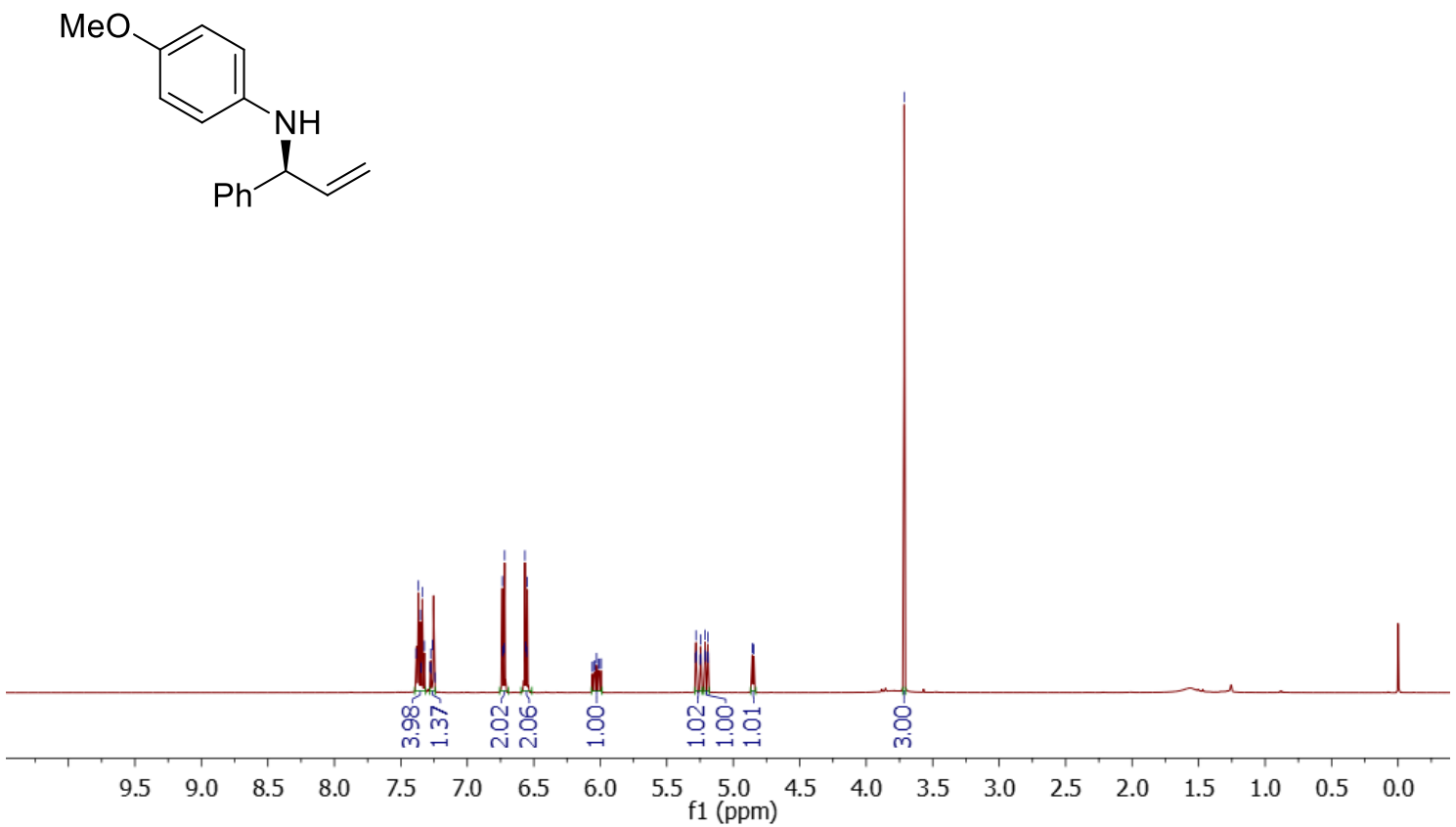

[3hb]

i

$\begin{array}{ll}0 & 0 \\ \text { ì } & 0 \\ 1 & 1\end{array}$

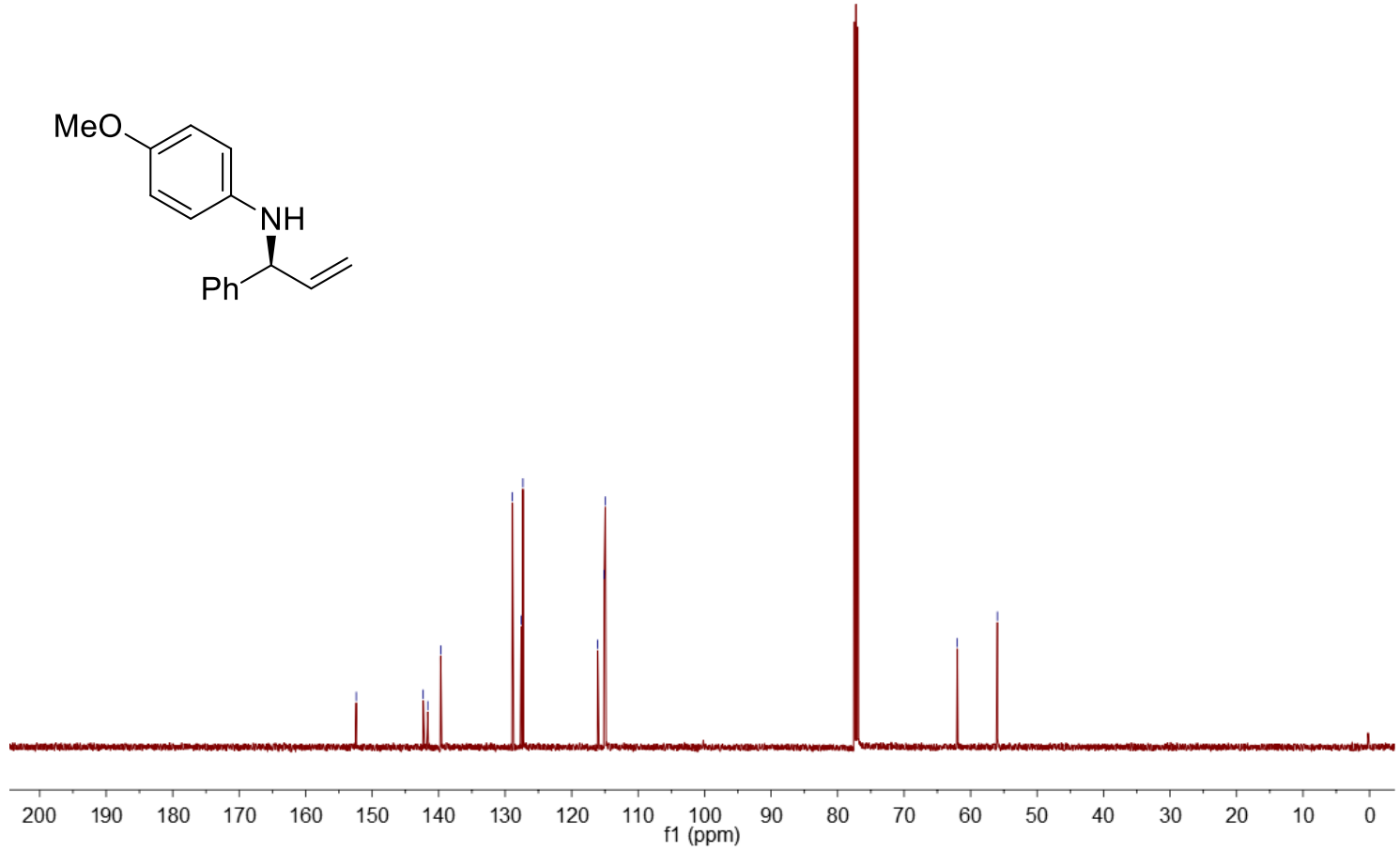




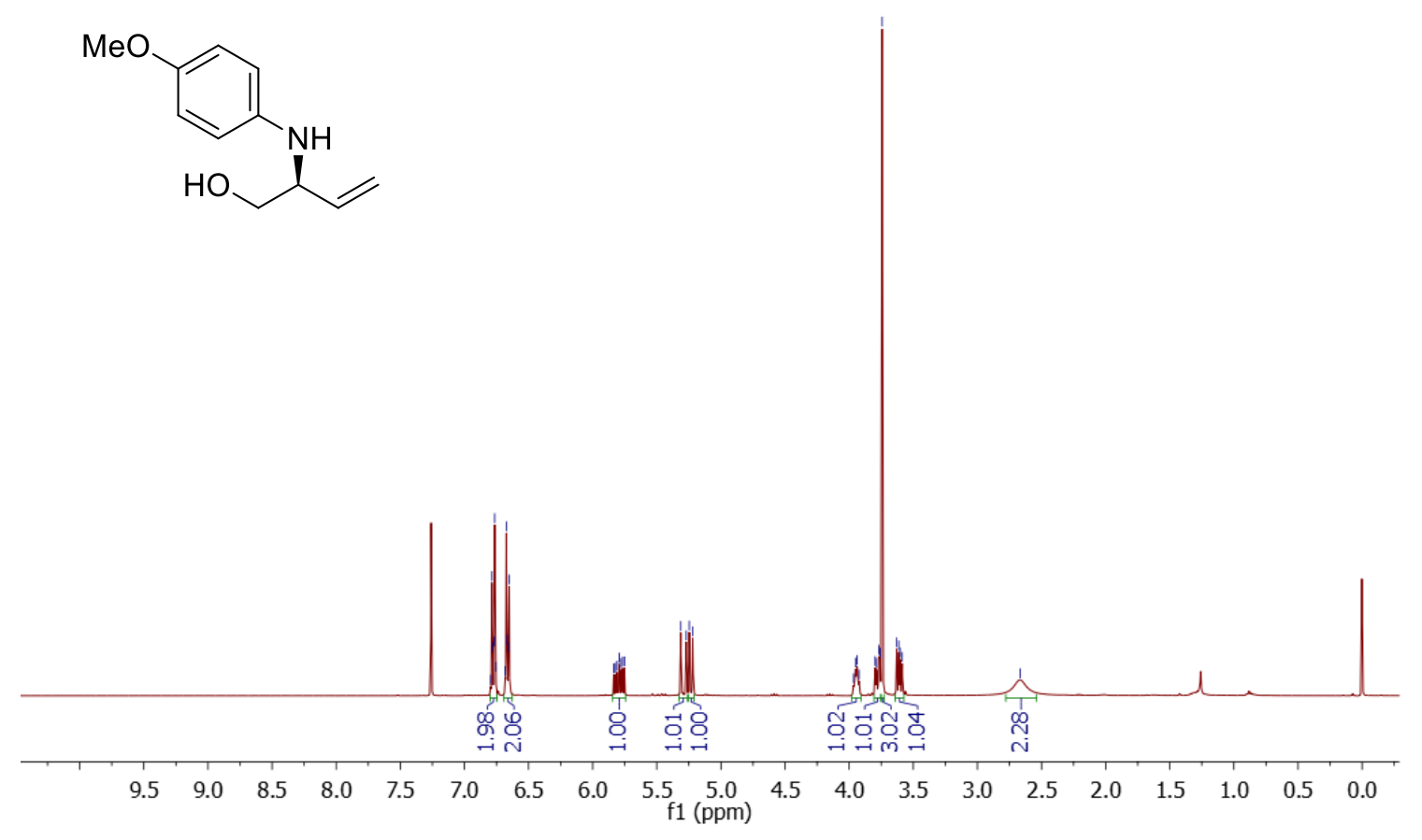

[3ib]

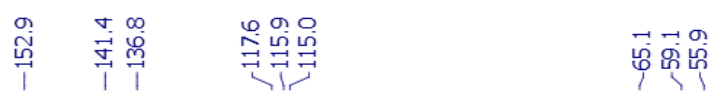

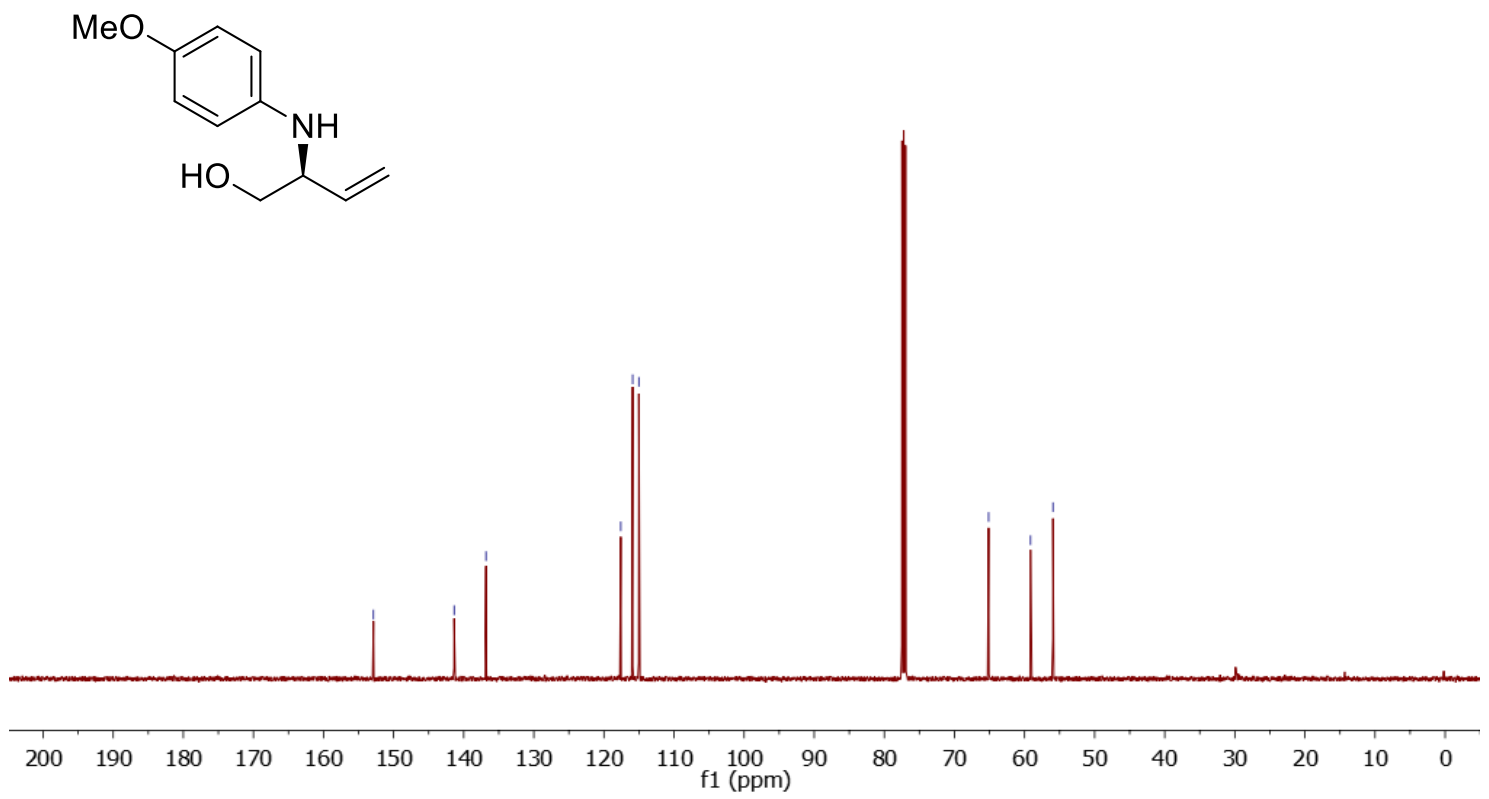




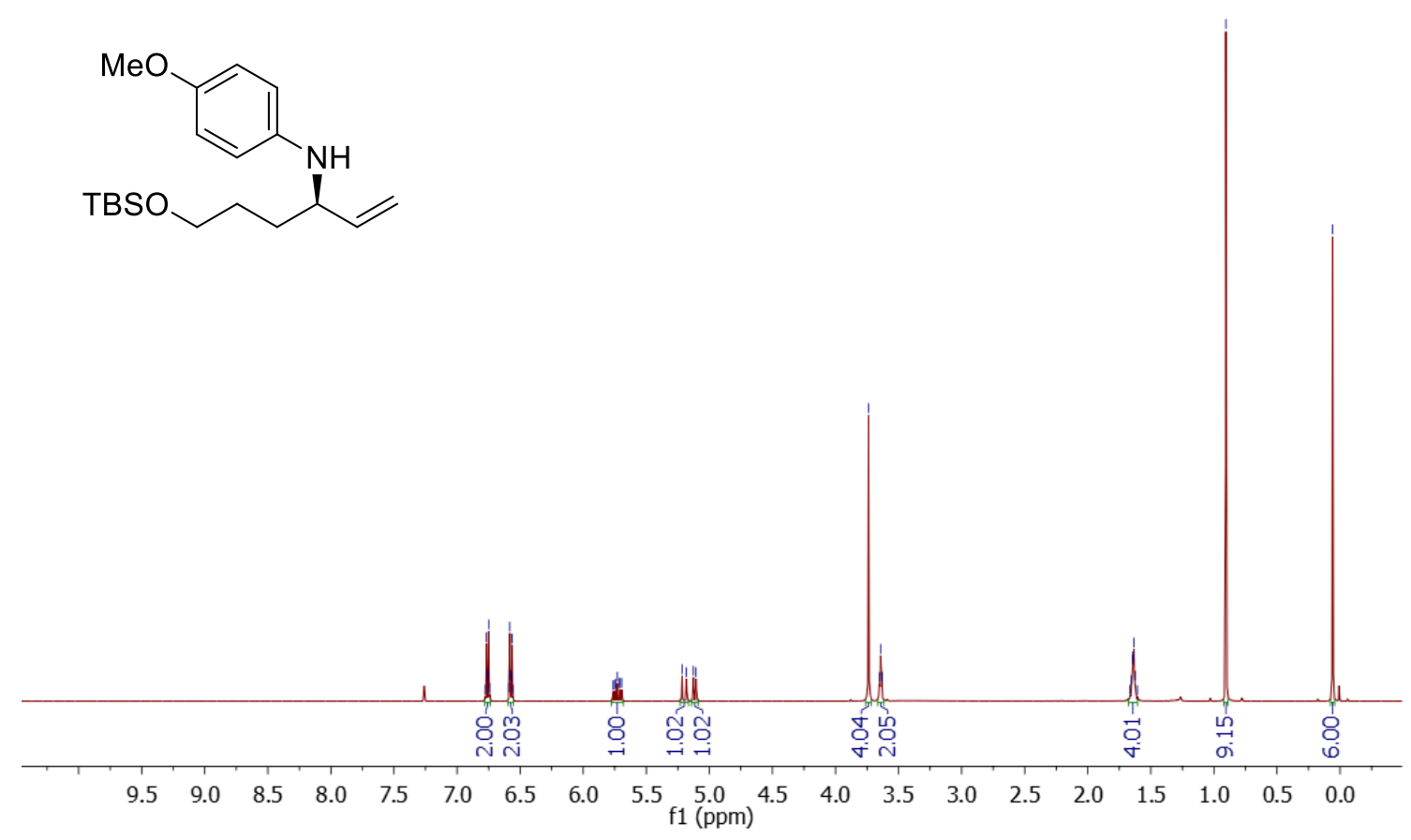

[3jb]
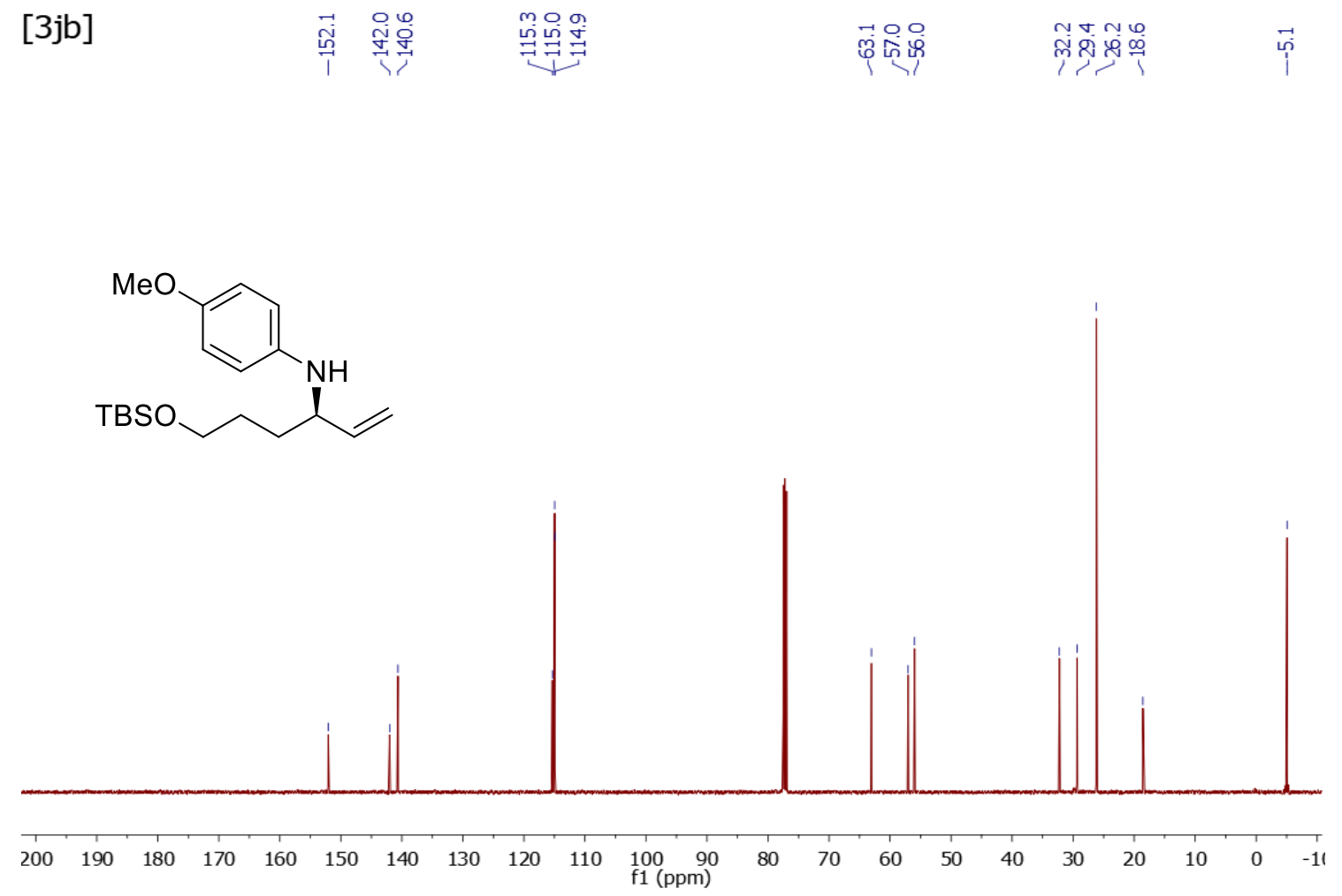


\section{[7ka]}

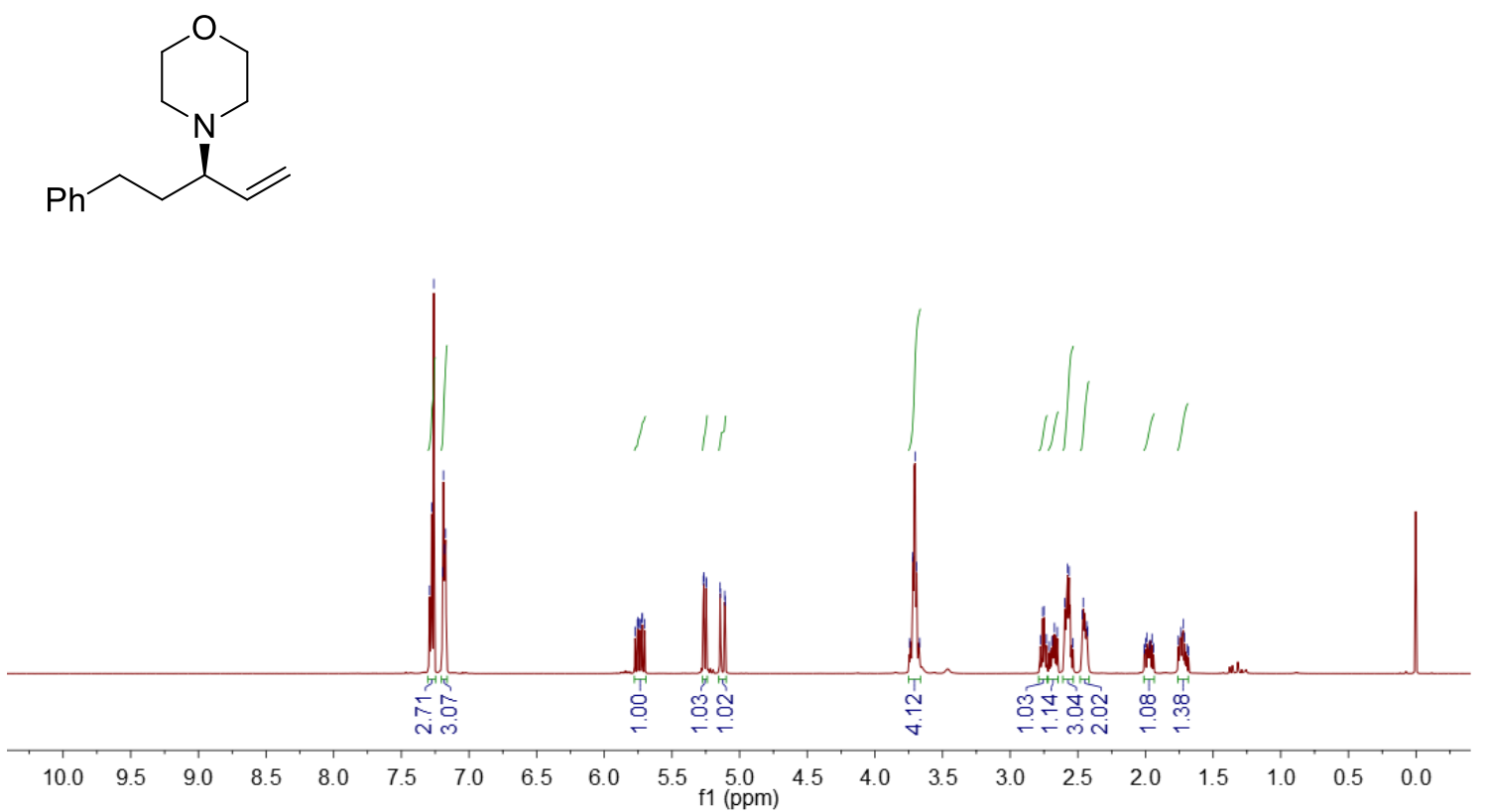

[7ka]



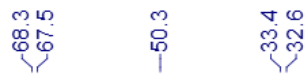<smiles>C=C[C@@H](CCc1ccccc1)N1CCOCC1</smiles>

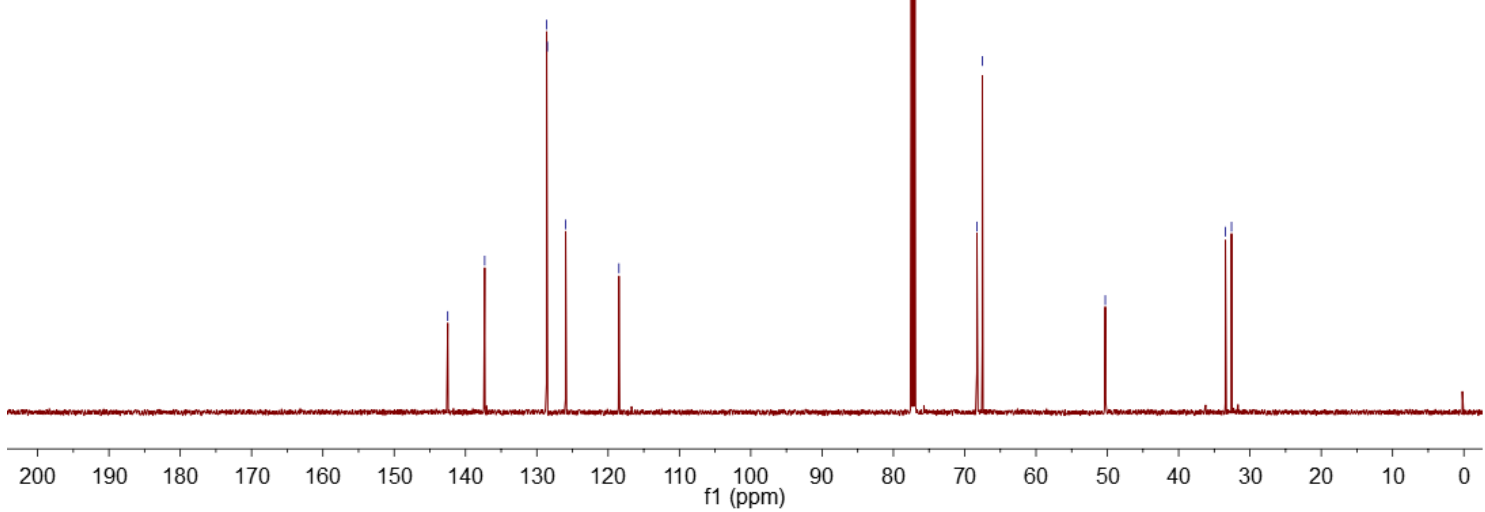


[7kb]

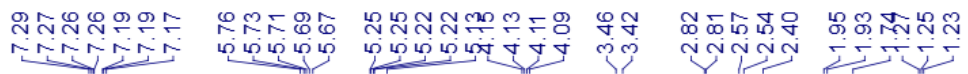

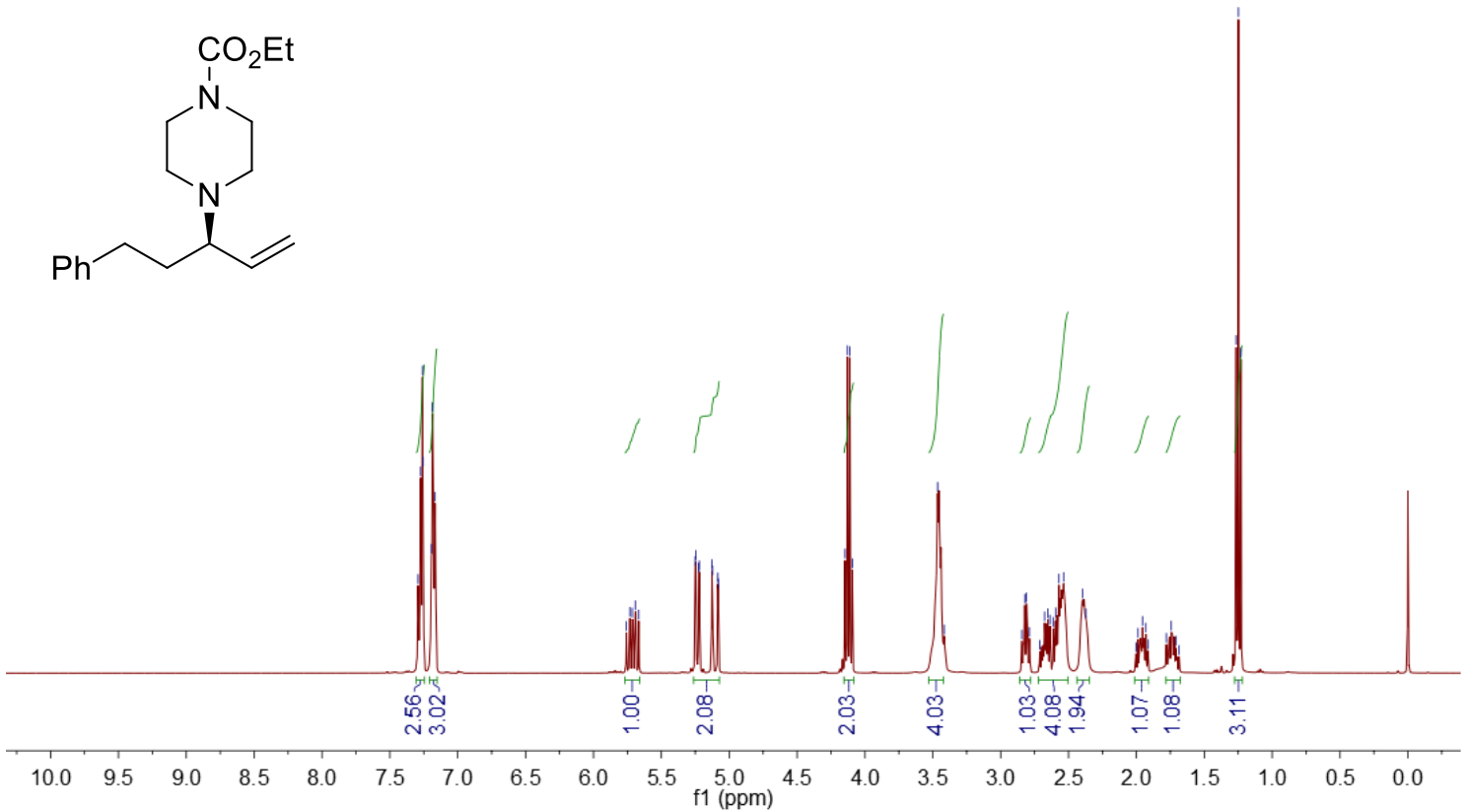

[7kb]

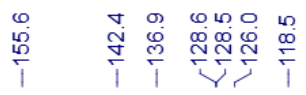

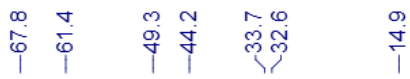

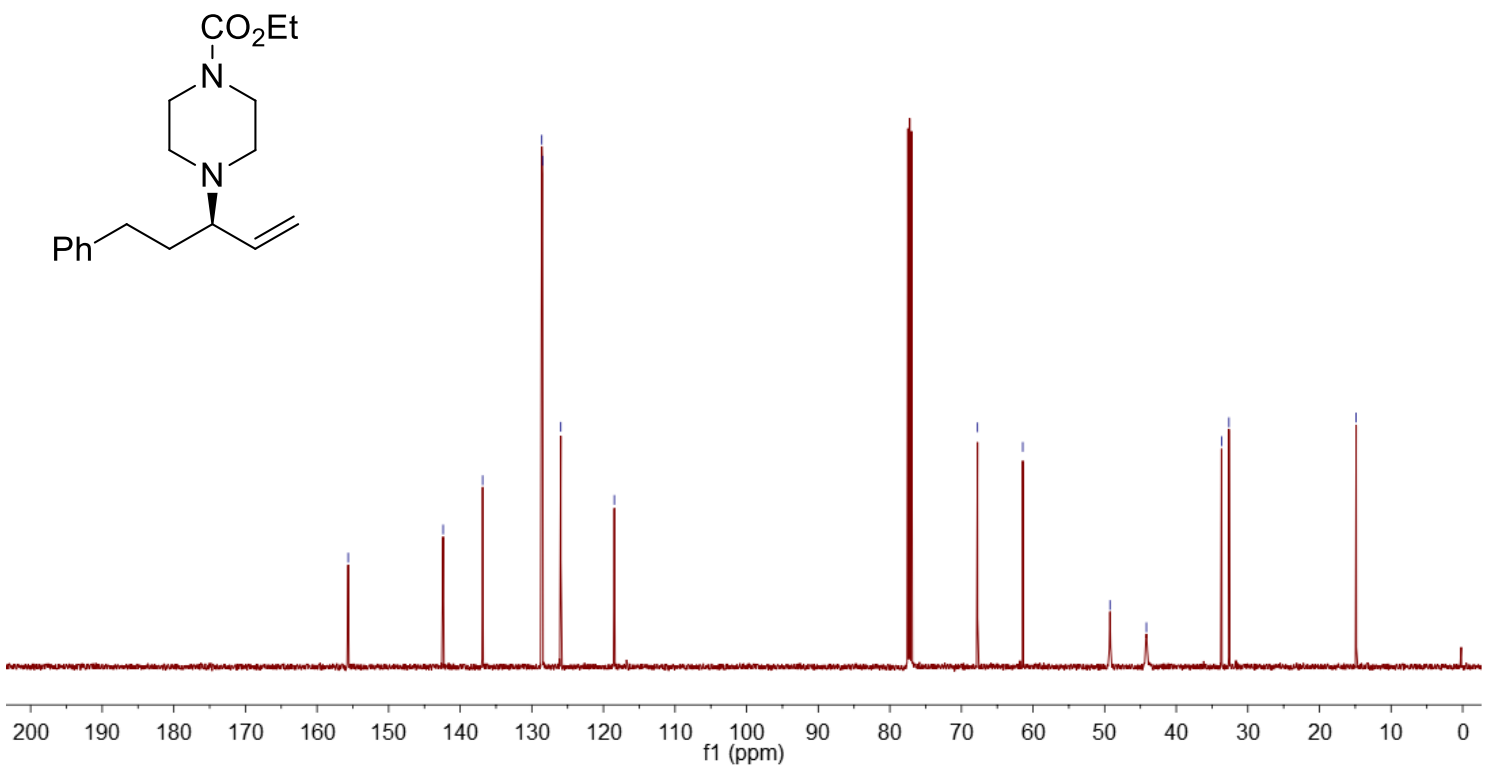


$[7 \mathrm{kc}]$

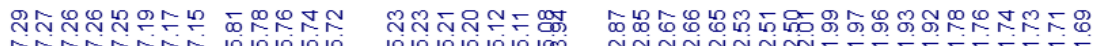

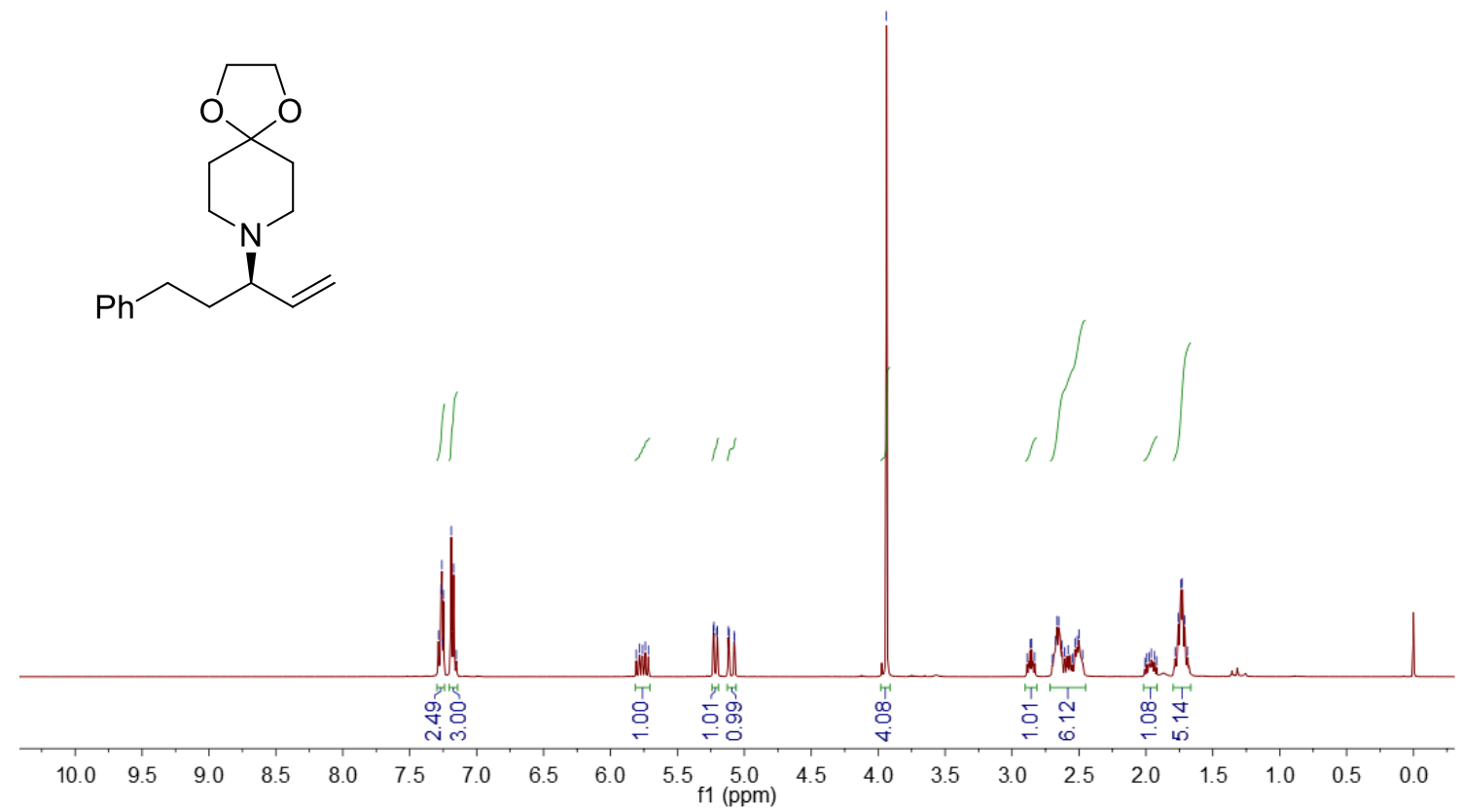

$[7 \mathrm{kc}]$

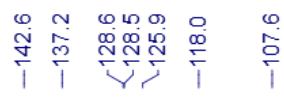

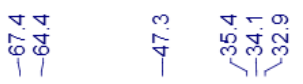

$\stackrel{1}{i}$

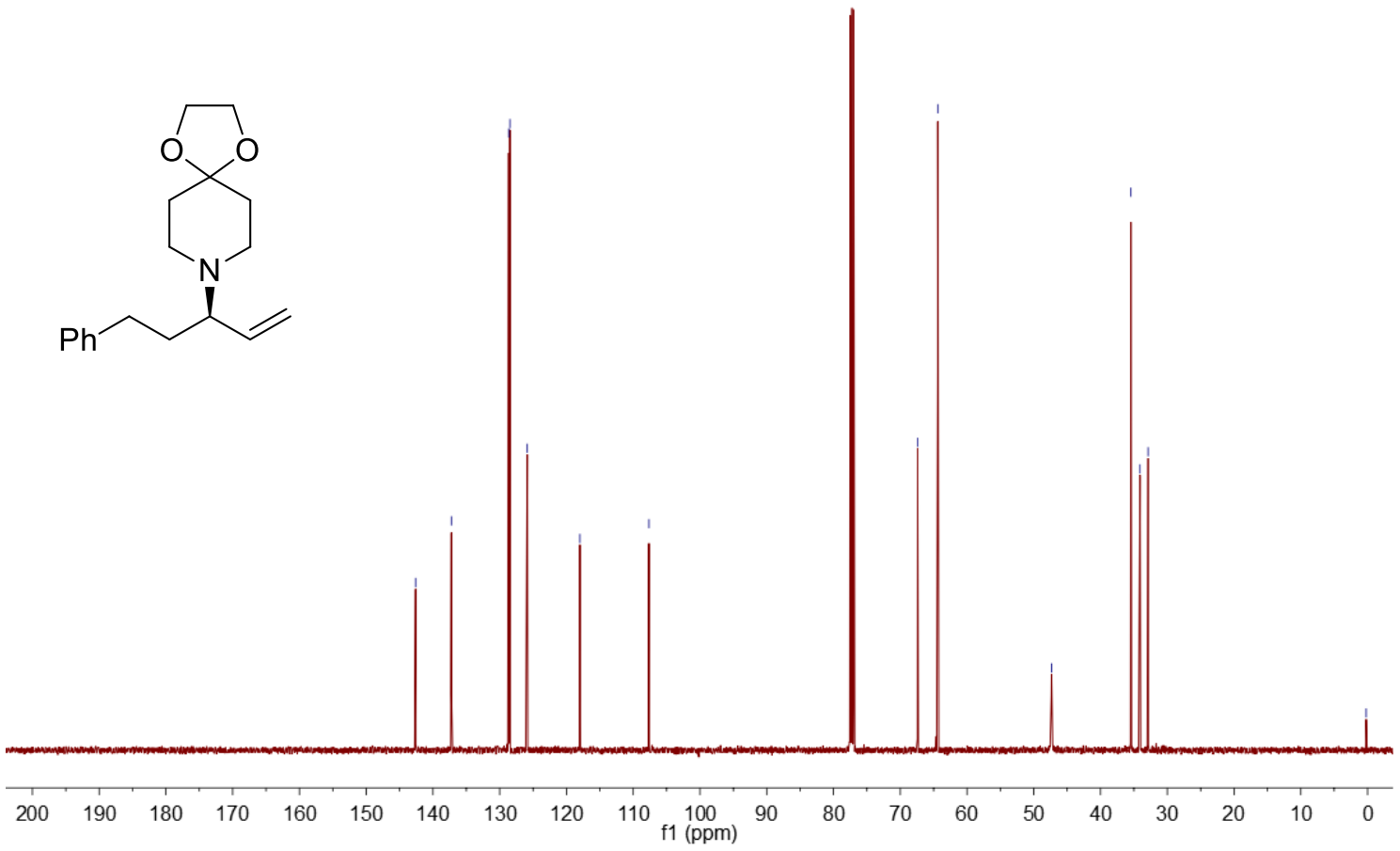



[7kd]

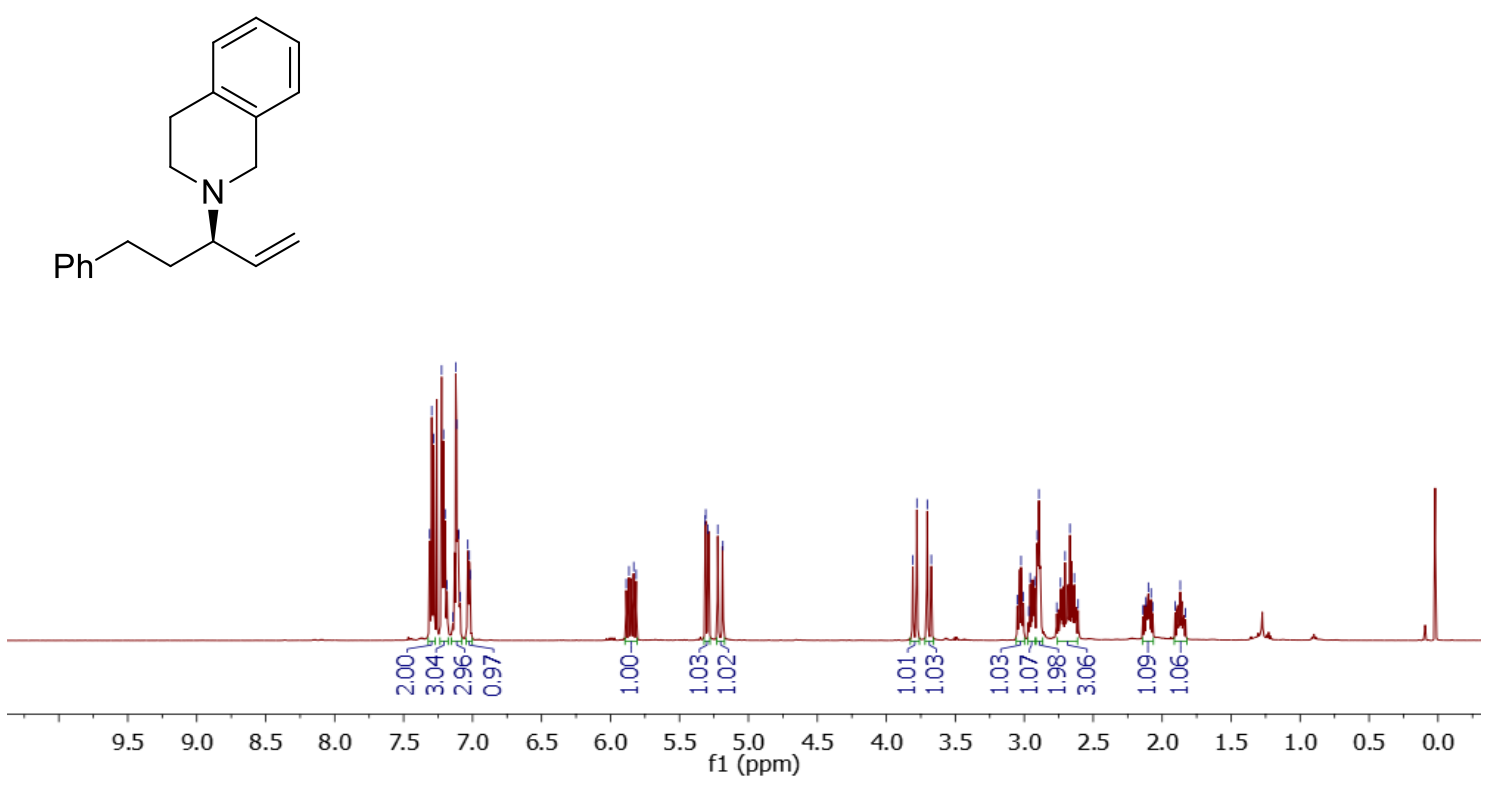

[7kd]

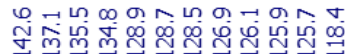

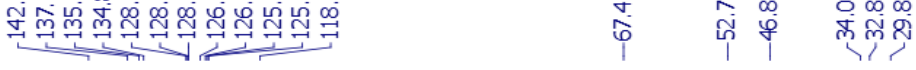

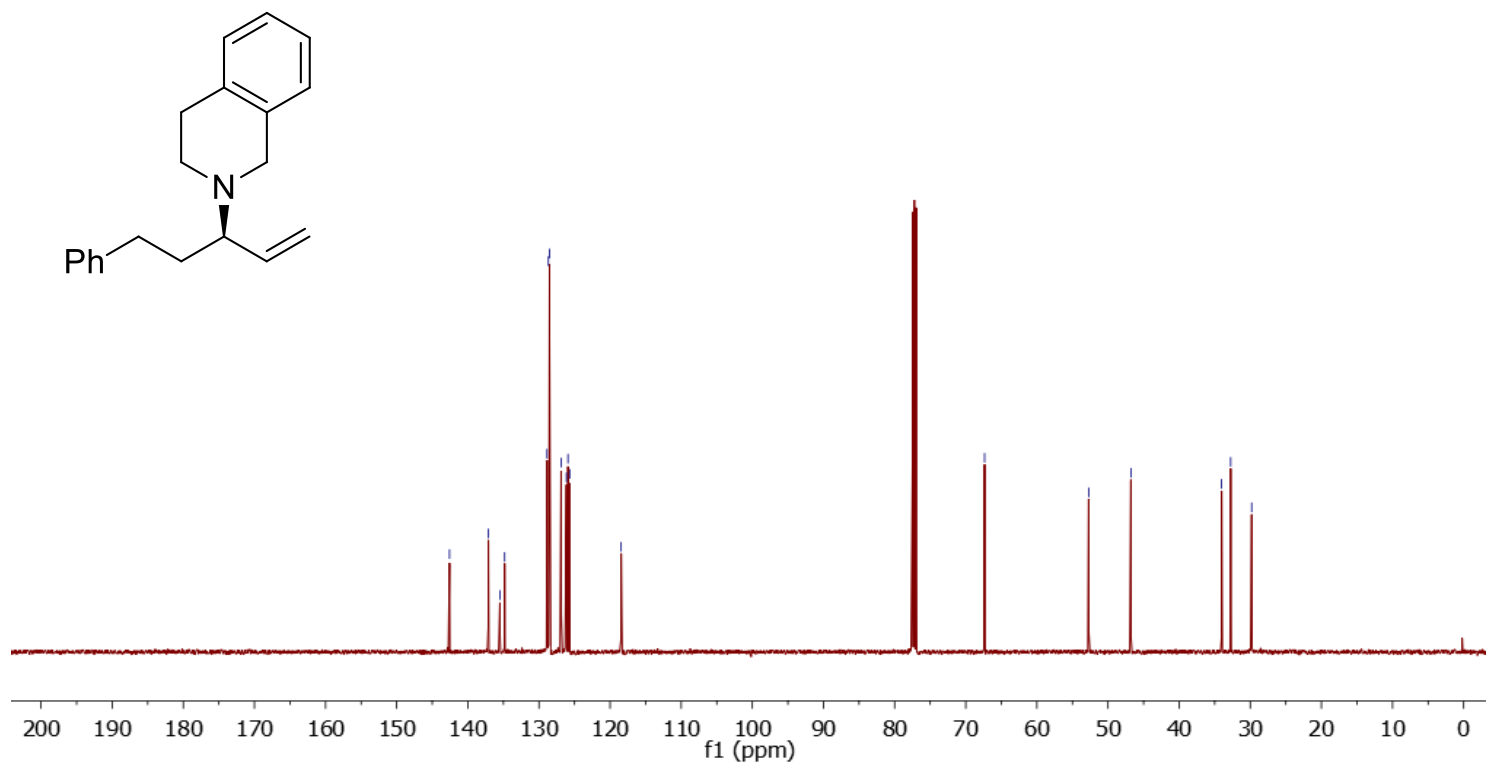


[7ke]
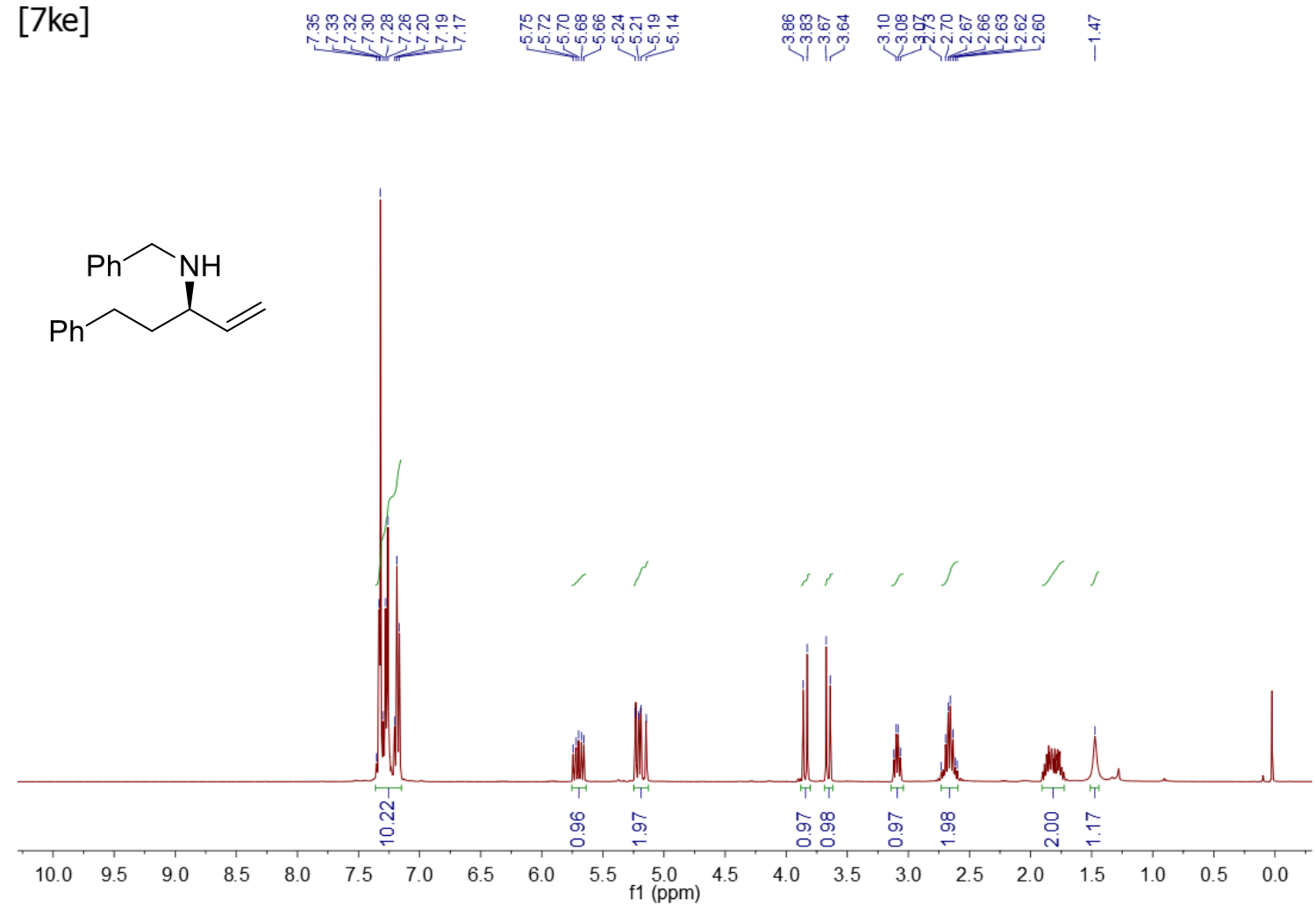

[7ke]

|خ

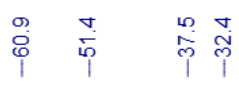
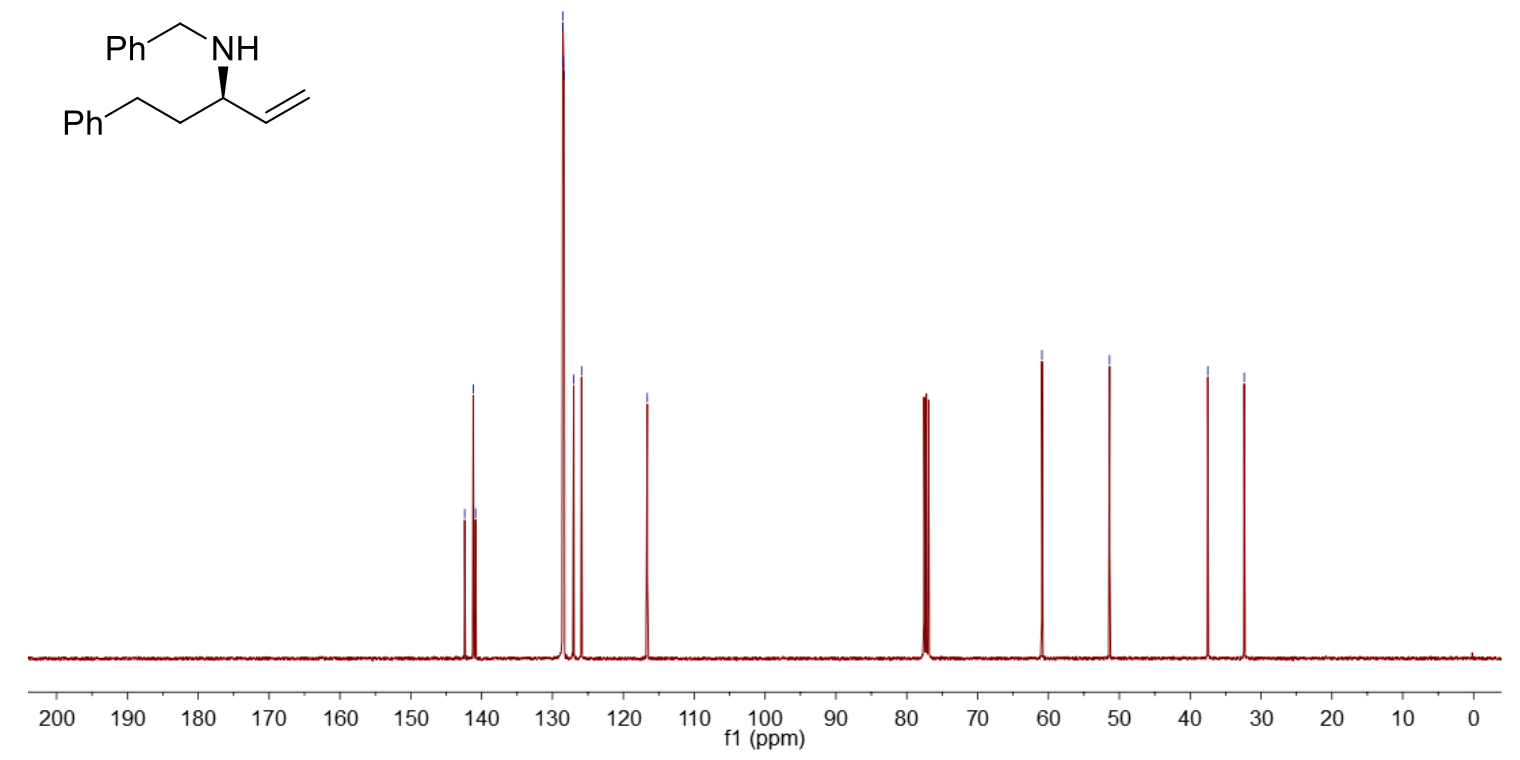
<smiles>C=C[C@H](CCc1ccccc1)NCCc1ccccc1</smiles>

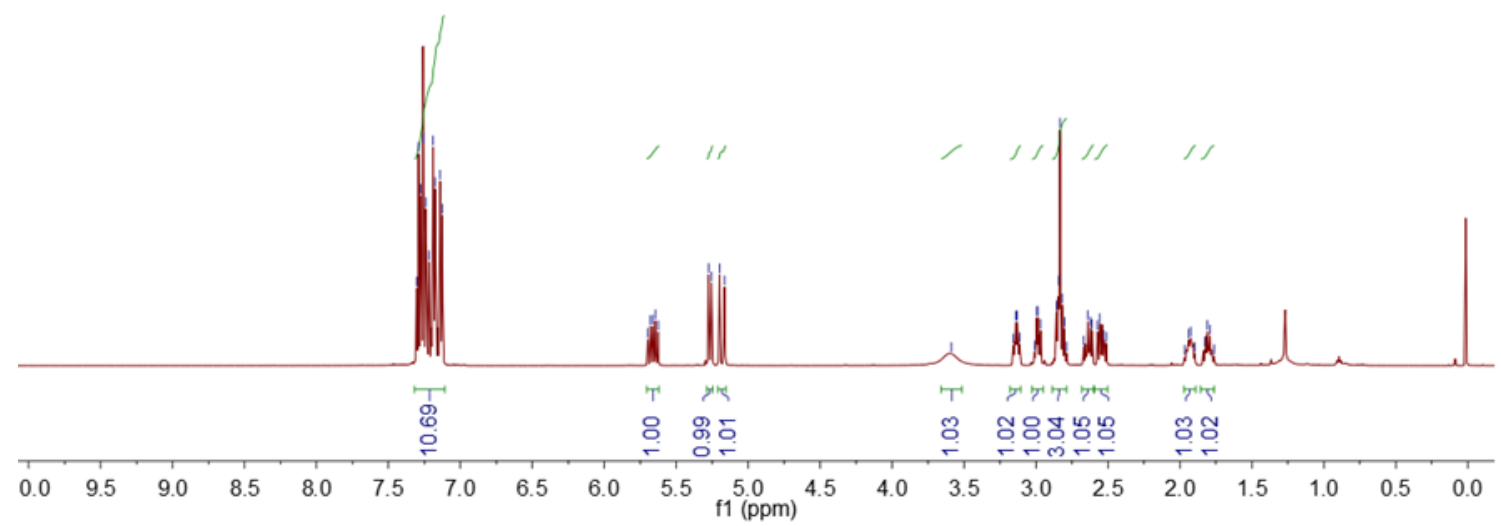

[7kf]<smiles>C=C[C@H](CCc1ccccc1)NCCc1ccccc1</smiles>

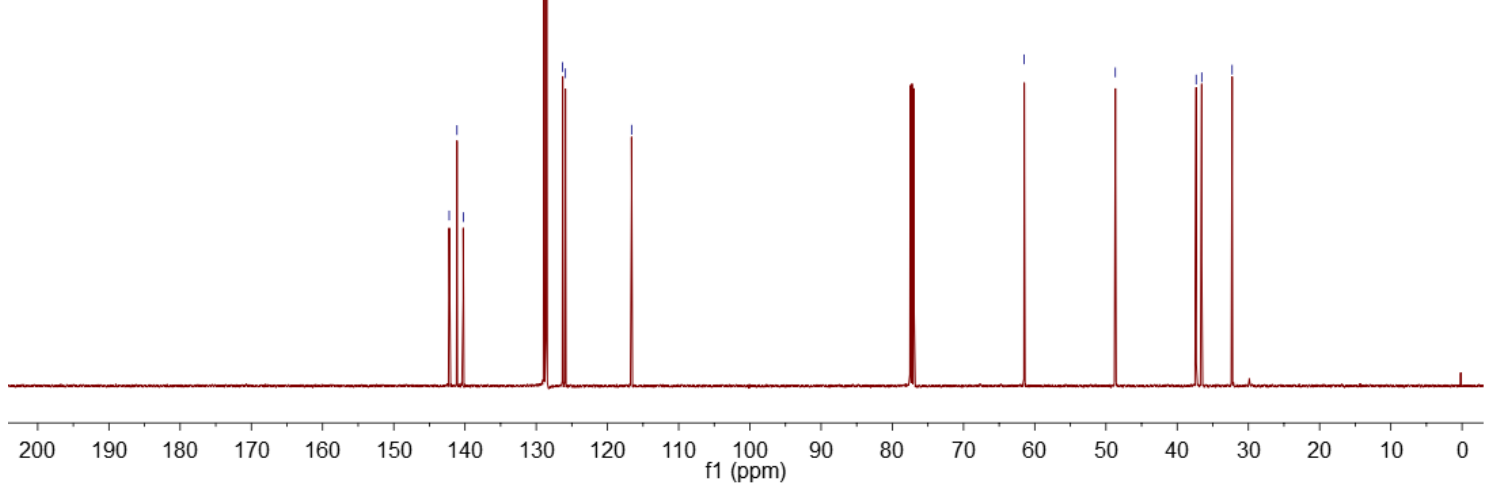


$[7 \mathrm{~kg}]$

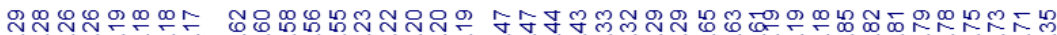

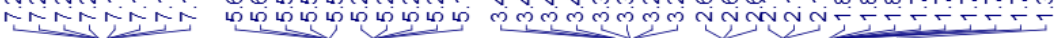<smiles>C#CCN[C@@H](C=C)CCc1ccccc1</smiles>

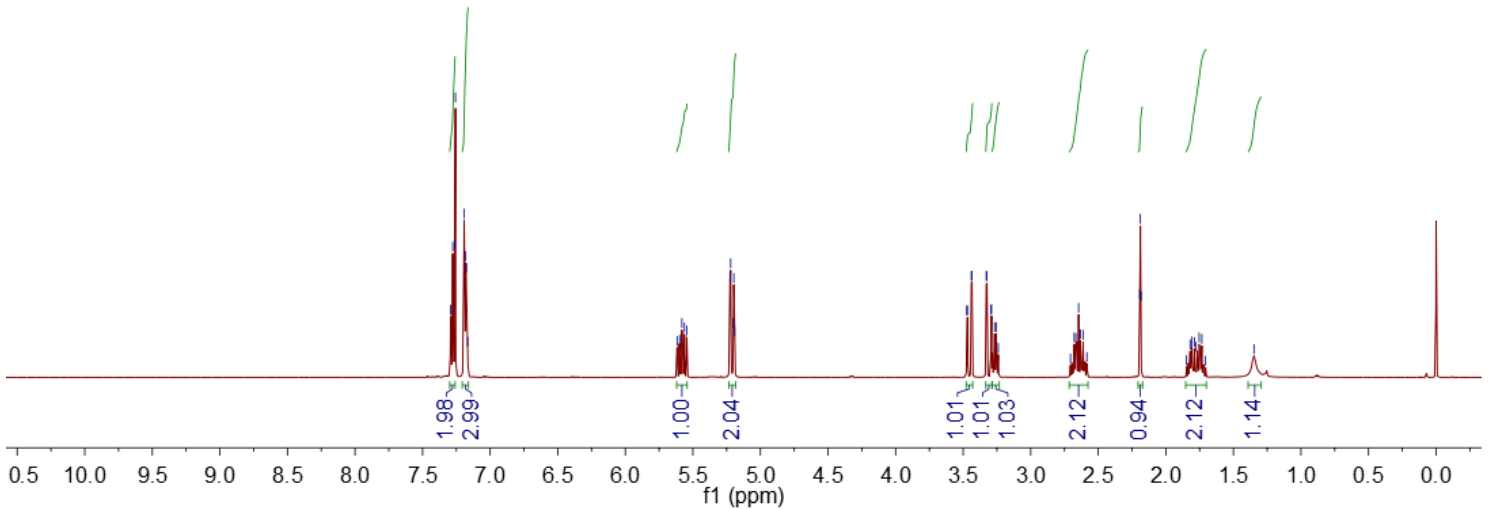

$[7 \mathrm{~kg}]$

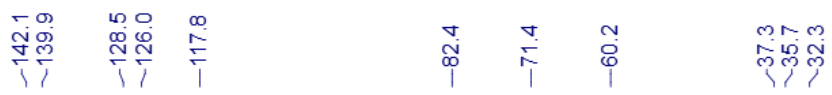
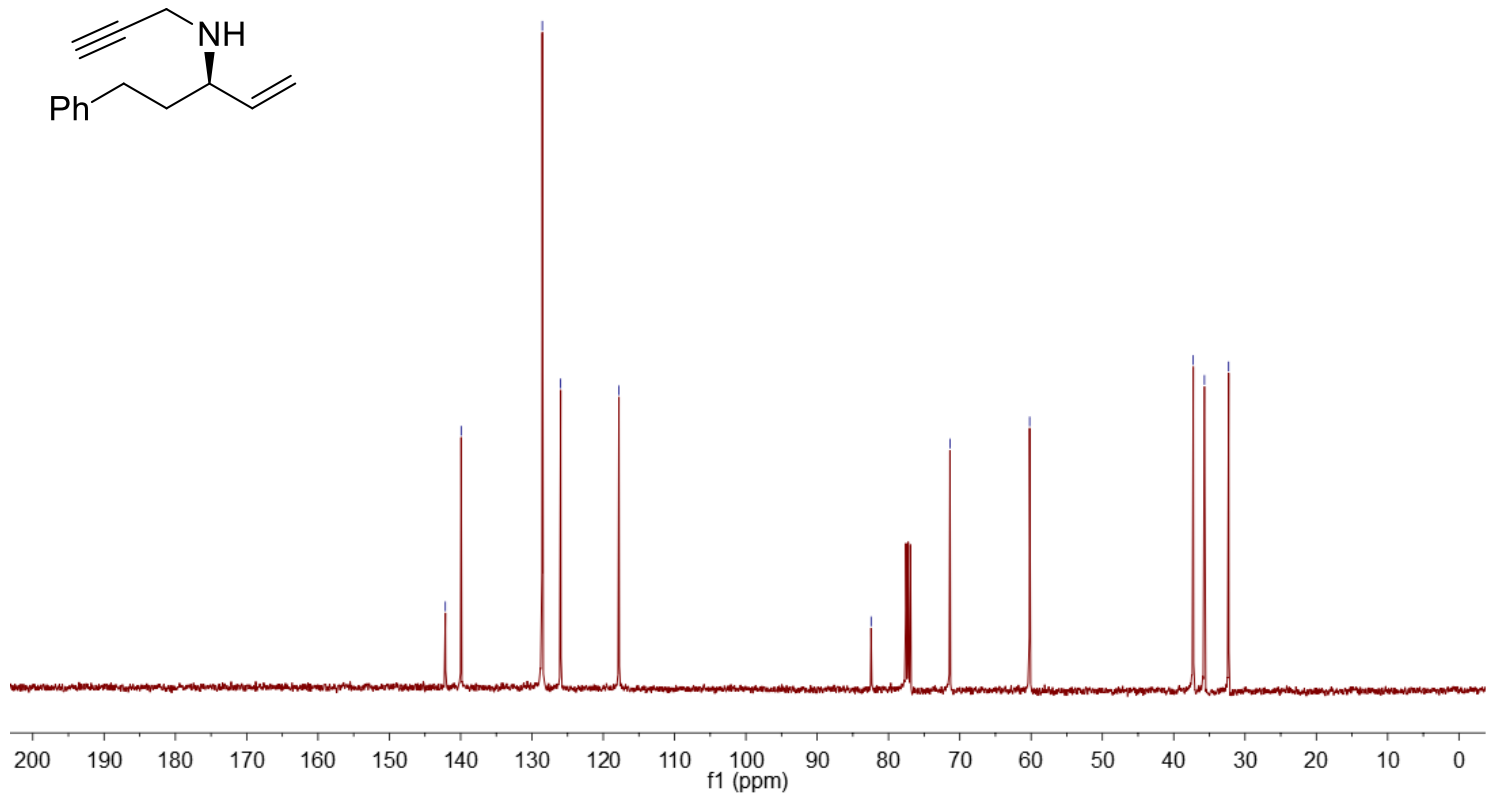


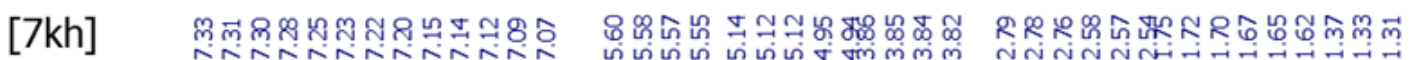

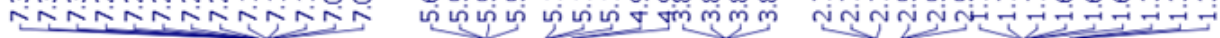

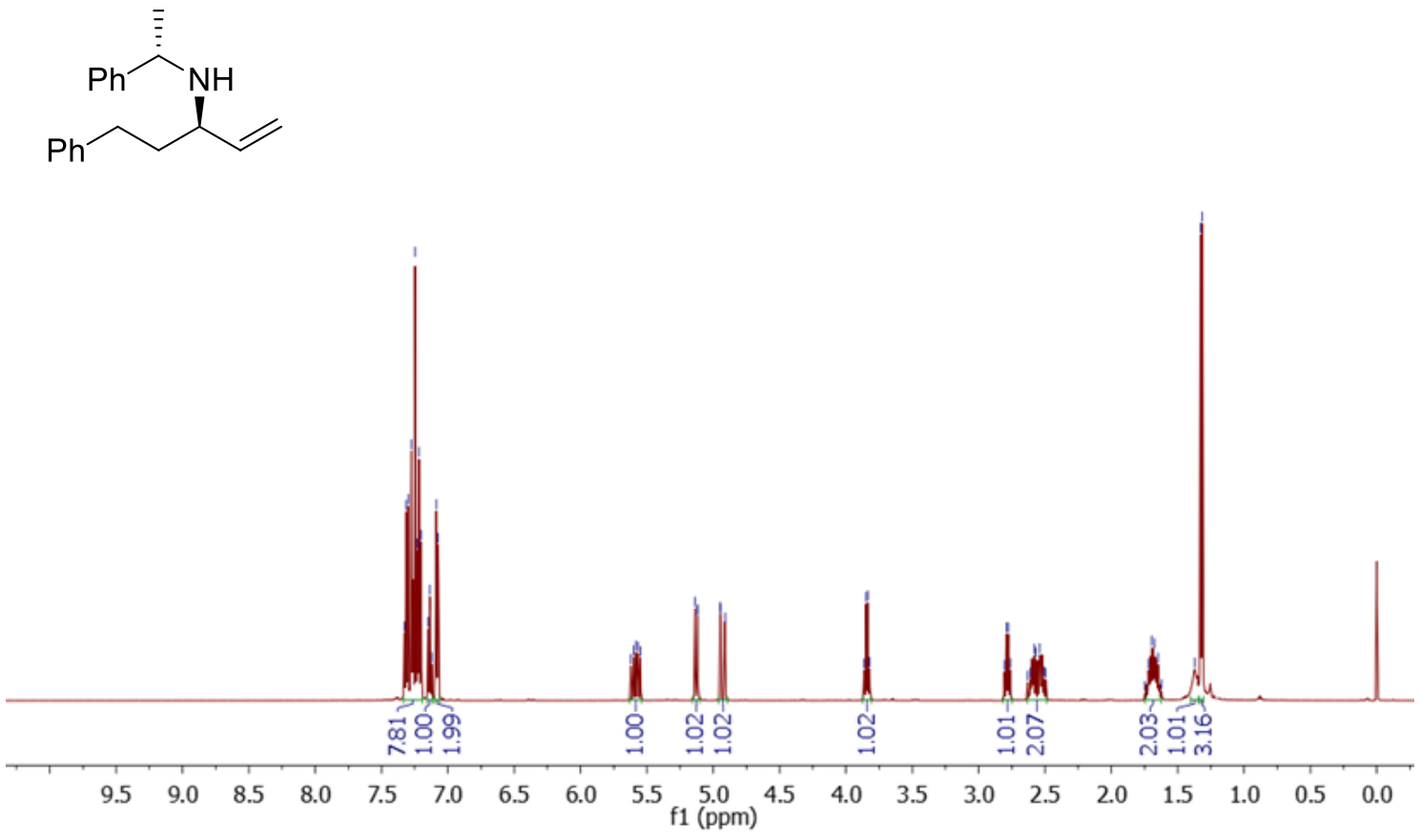

$[7 \mathrm{kh}]$

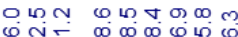

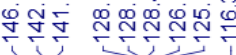

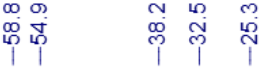

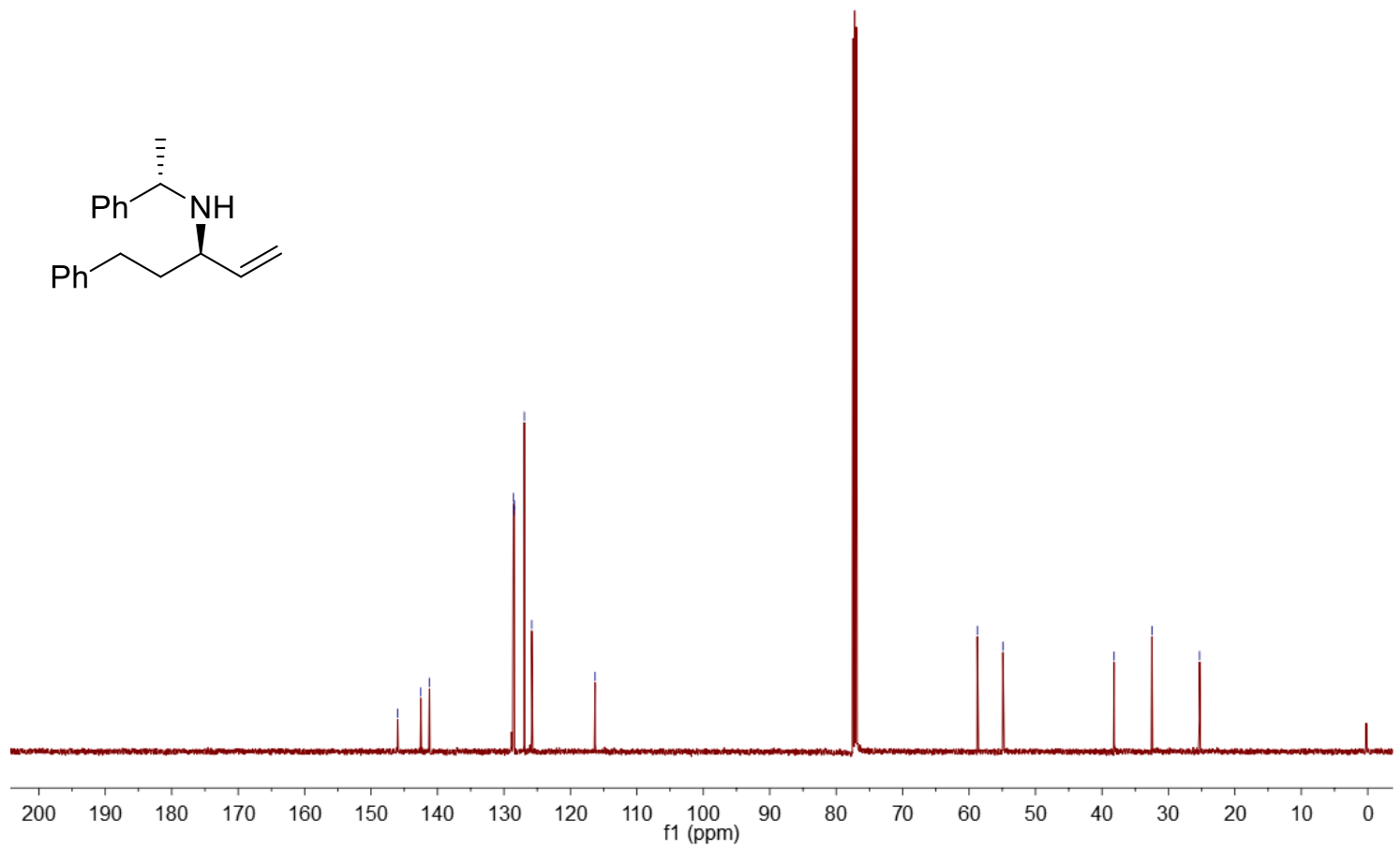




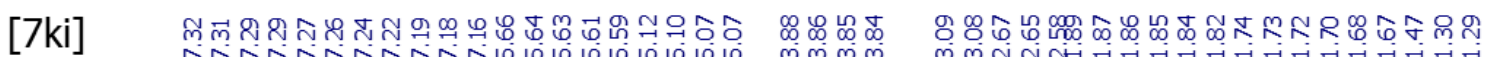

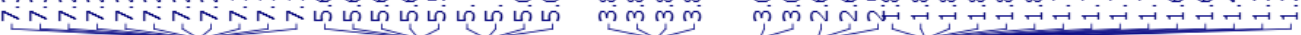

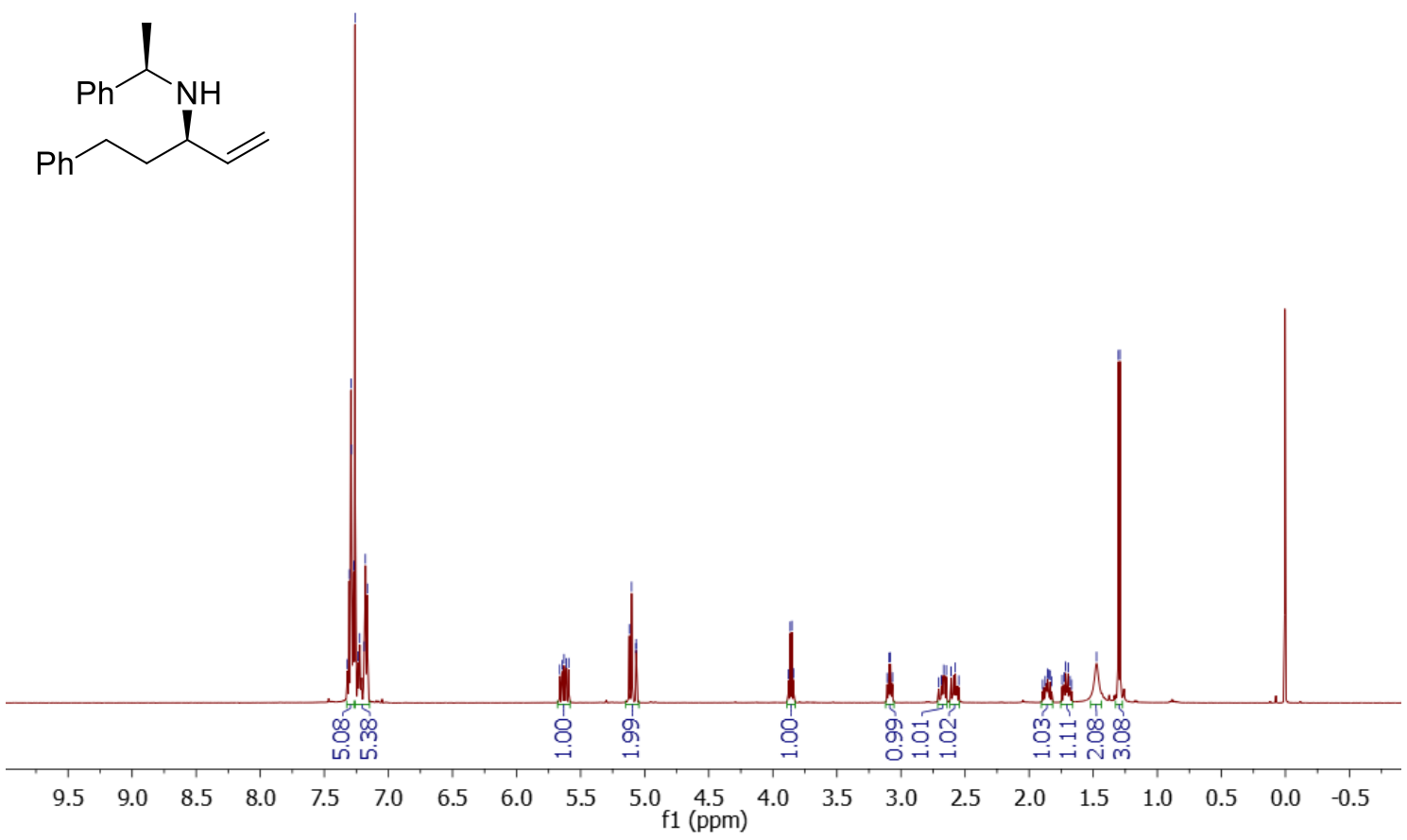

[7ki]

눙

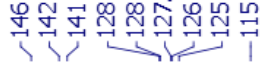

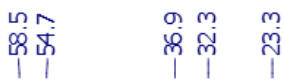
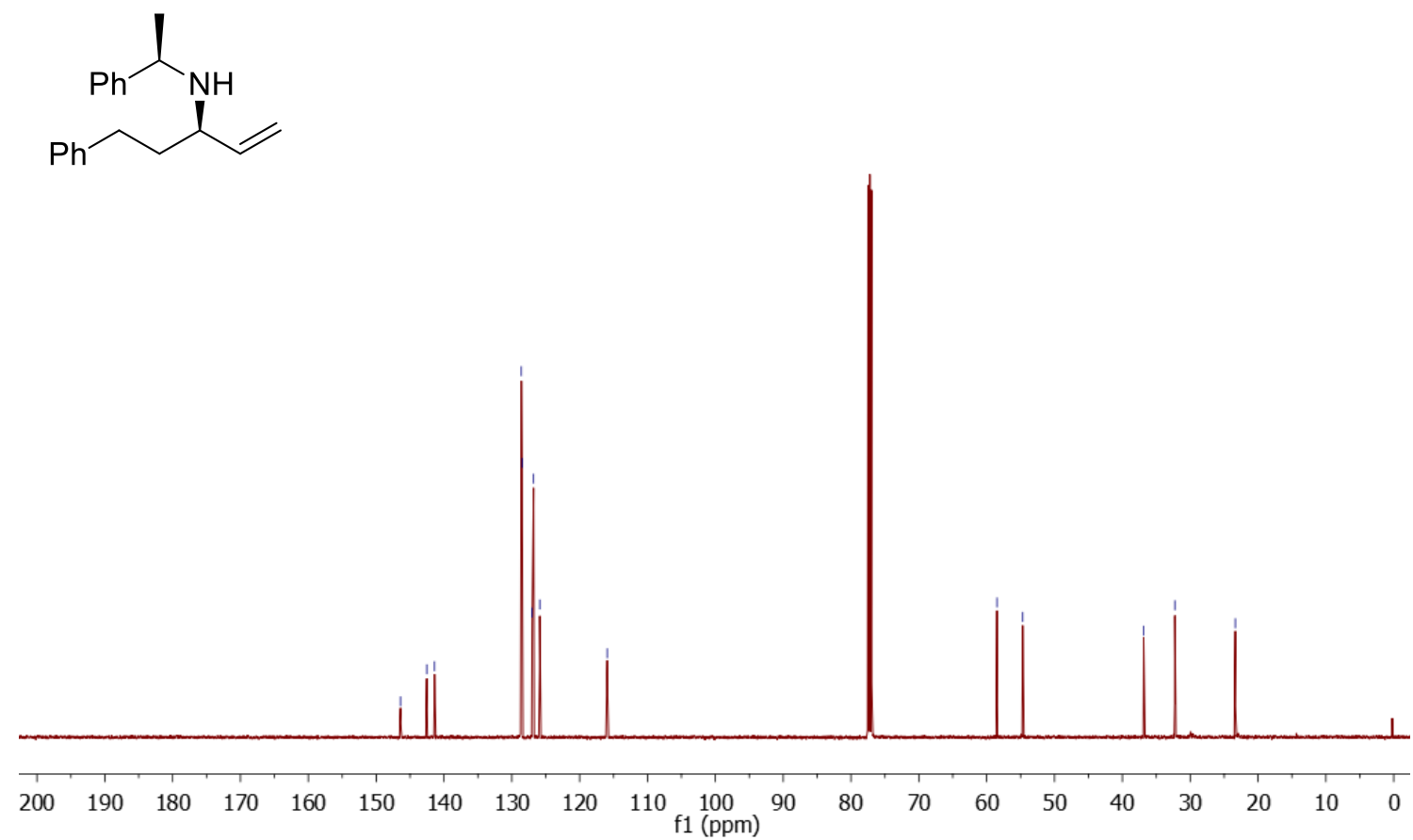
[7kj]

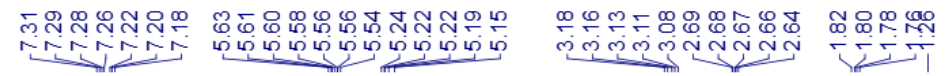<smiles>C=C[C@H](CCc1ccccc1)NCC(F)(F)F</smiles>

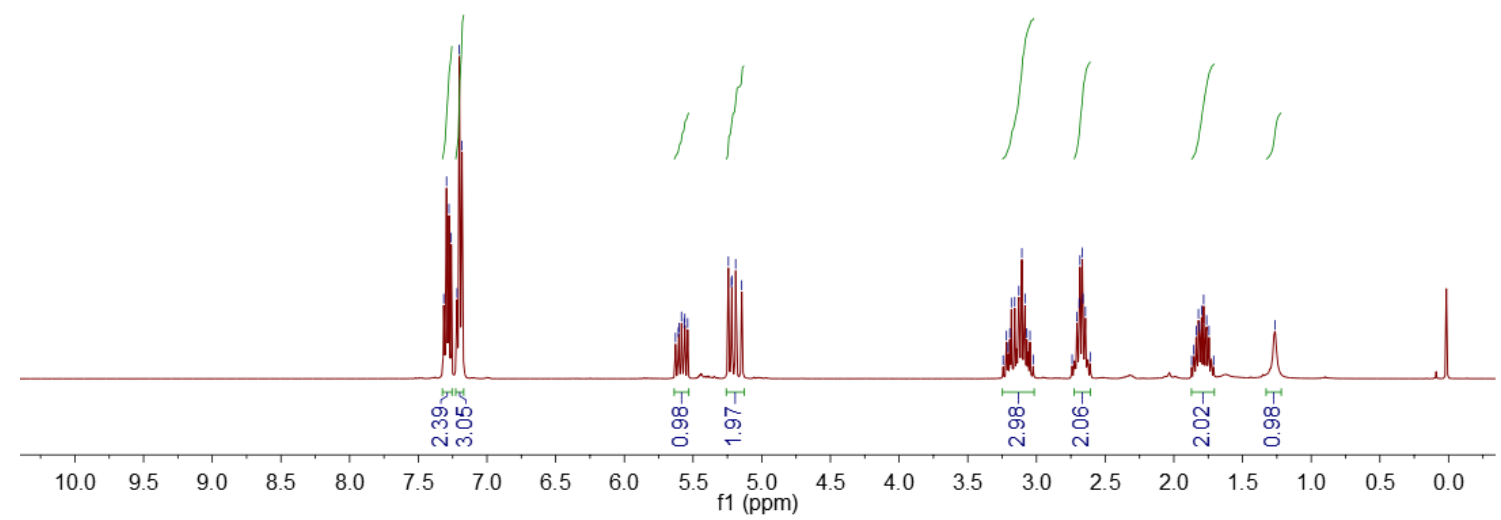

[7kj]

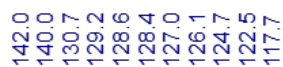

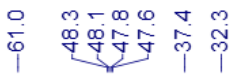

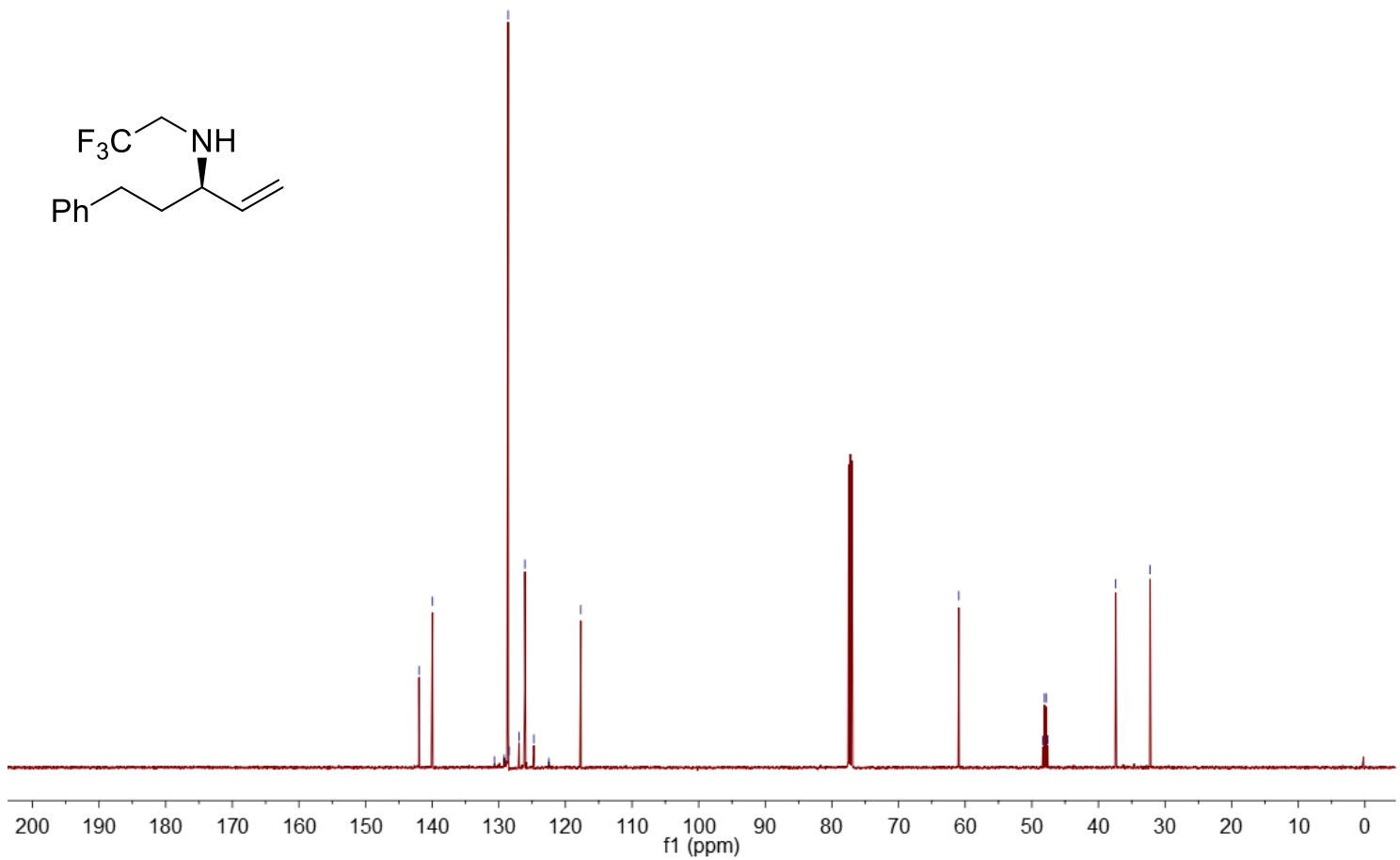




\section{HPLC spectra of chiral compounds:}

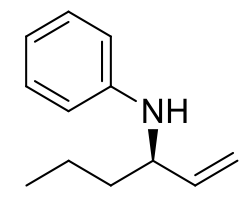

3aa

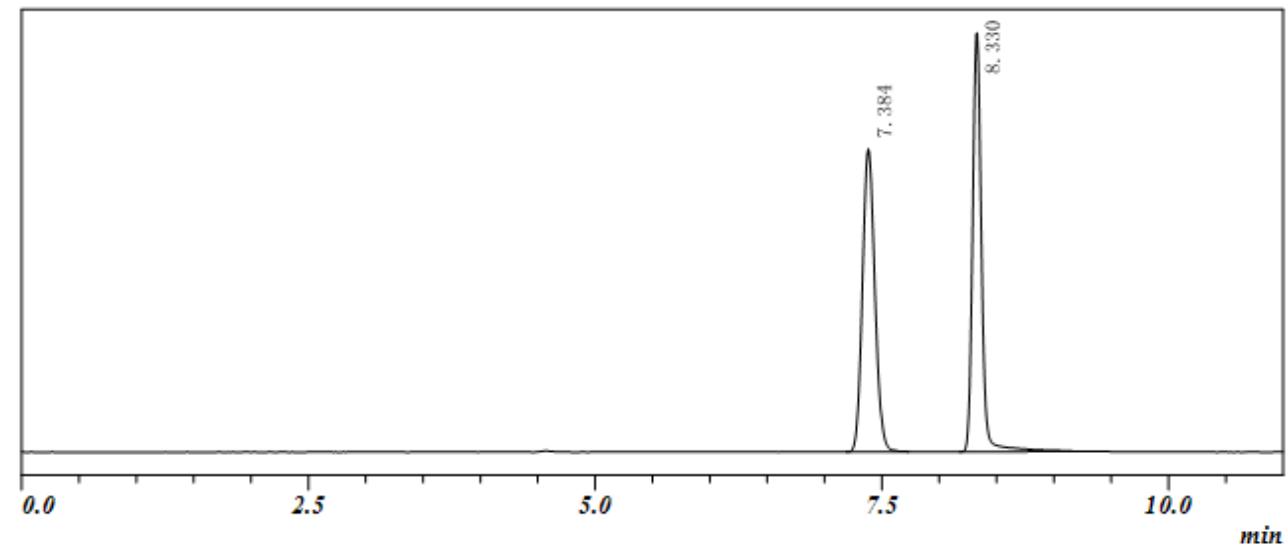

PEAK TABLE

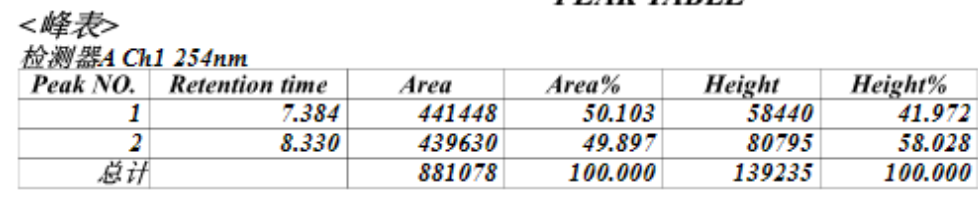

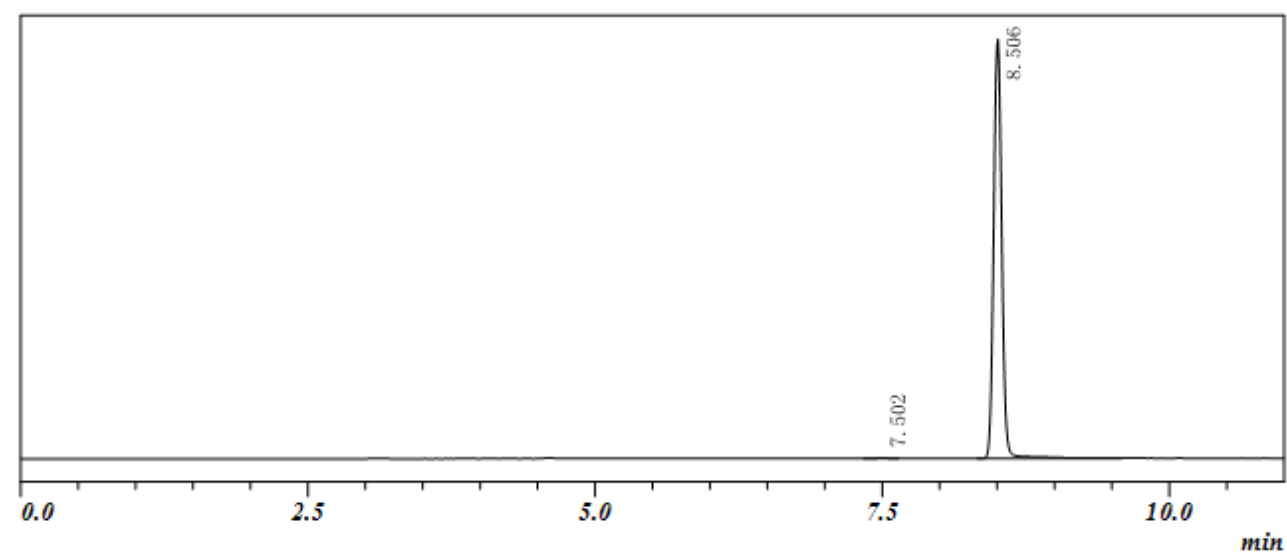

PEAK TABLE

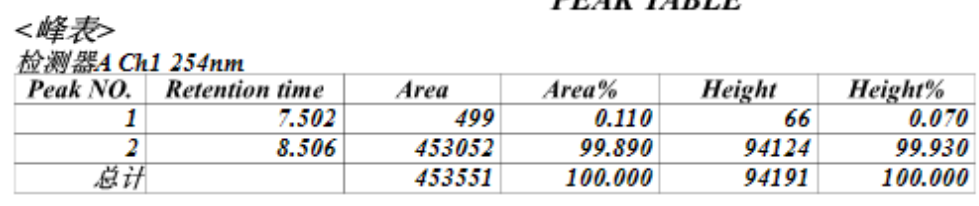




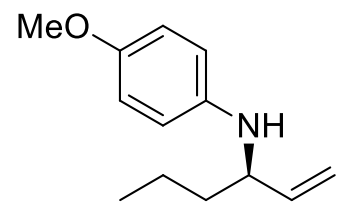

$3 a b$

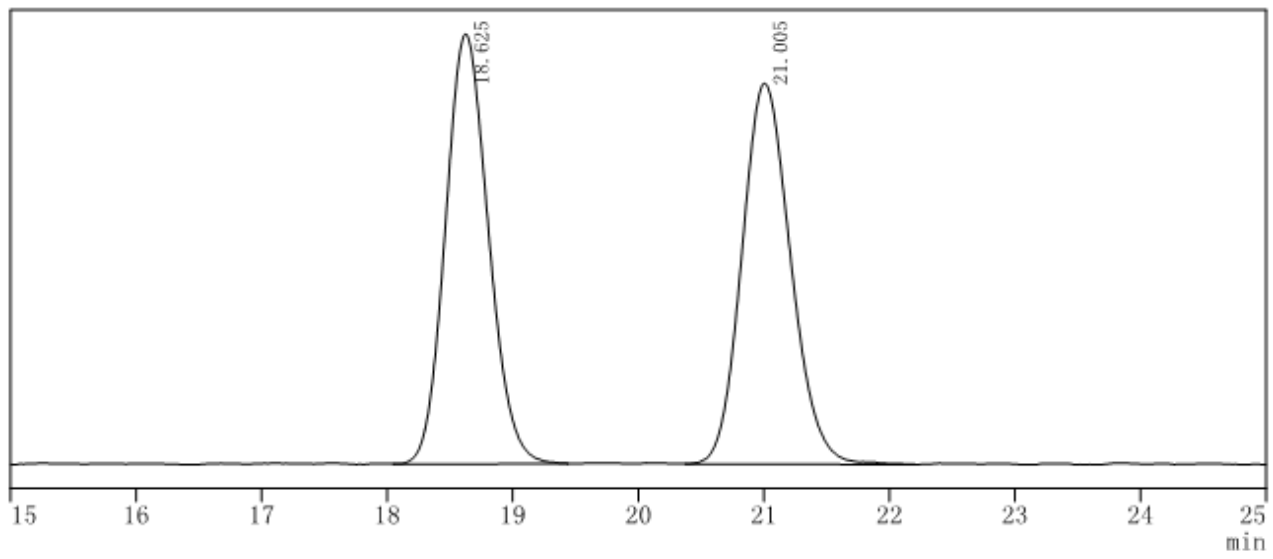

检测器A Ch1 $254 n m$

\begin{tabular}{|r|r|r|r|r|r|} 
Peak NO. & Retension Time & Area & Area\% & Height & \multicolumn{1}{|c|}{ Height\% } \\
1 & 18.625 & 359298 & 49.886 & 15056 & 53.033 \\
\hline 2 & 21.005 & 360935 & 50.114 & 13334 & 46.967 \\
\hline 总计 & & 720233 & 100.000 & 28391 & 100.000 \\
\hline
\end{tabular}

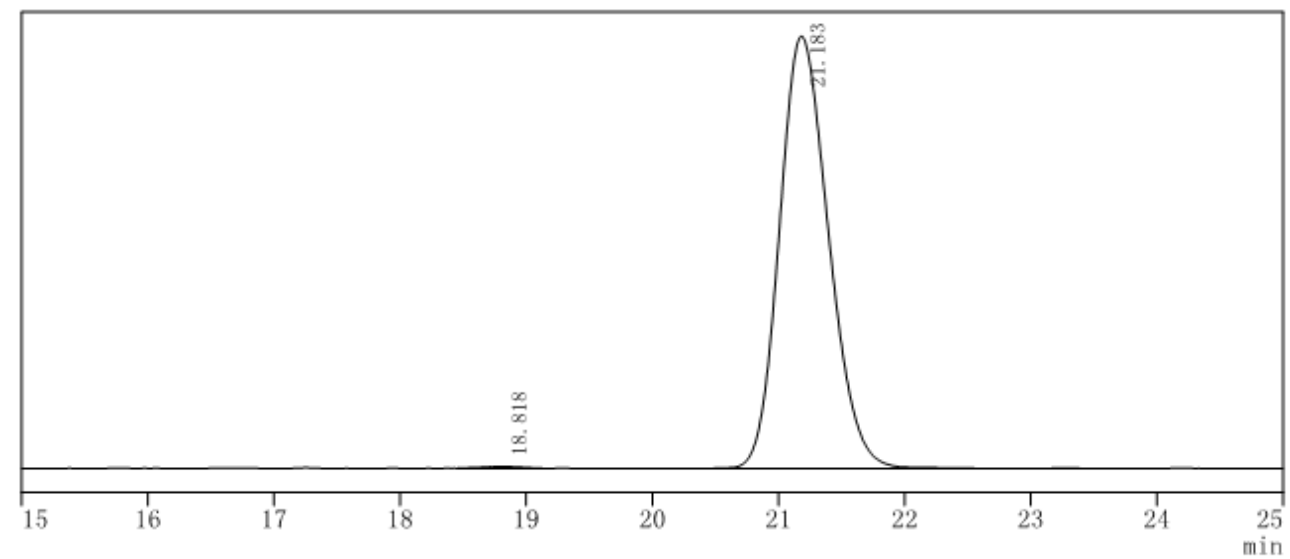

检测器ACh1 $254 \mathrm{~nm}$

\begin{tabular}{|r|r|r|r|r|r|}
\hline Peak NO. Retention Time & Height & Height\% & Area & \multicolumn{1}{|c|}{ Area\% } \\
1 & 18.818 & 242 & 0.468 & 5543 & 0.397 \\
\hline 2 & 21.183 & 51436 & 99.532 & 1391980 & 99.603 \\
\hline 总计 & & 51678 & 100.000 & 1397524 & 100.000 \\
\hline
\end{tabular}




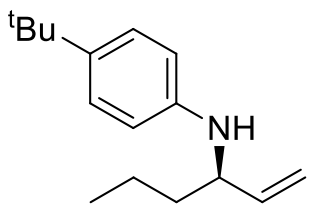

3ac

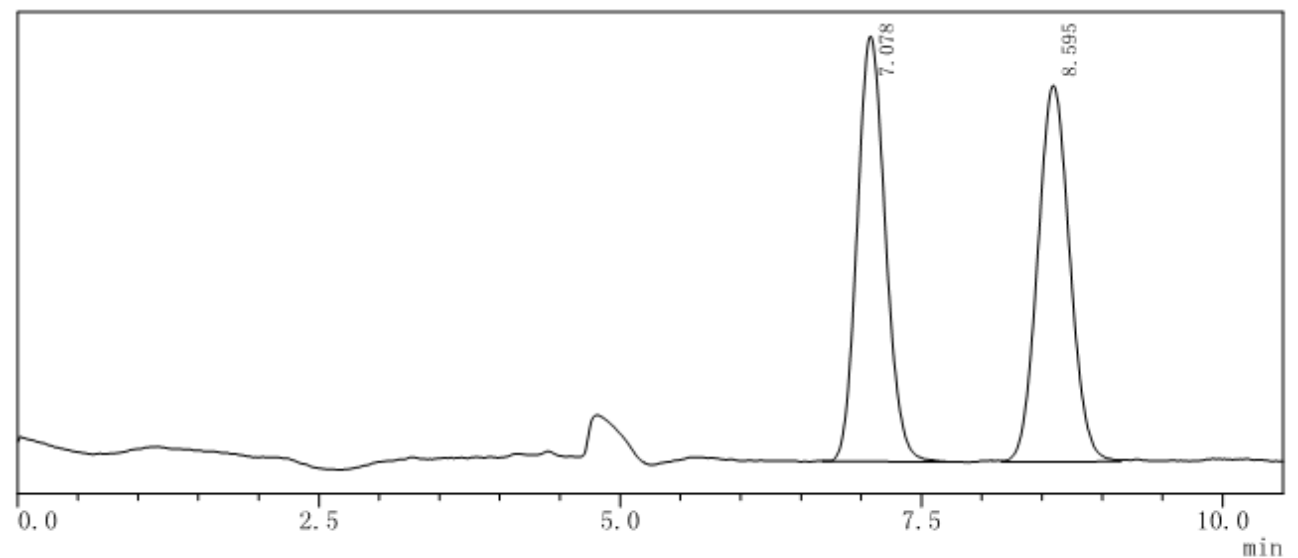

\begin{tabular}{|c|c|c|c|c|c|}
\hline${ }_{\text {Peak NO. }}$ & Retention Time & $\begin{array}{l}\text { Height } \\
8143\end{array}$ & $\begin{array}{l}\text { Height } \% \\
53.054\end{array}$ & $\begin{array}{l}\text { Area } \\
\quad 132974\end{array}$ & $\begin{array}{l}\text { Area } \% \\
49.919\end{array}$ \\
\hline & 8.595 & 7206 & 46.946 & 133406 & 50.081 \\
\hline D & & 15349 & 100.000 & 266380 & 100.000 \\
\hline
\end{tabular}

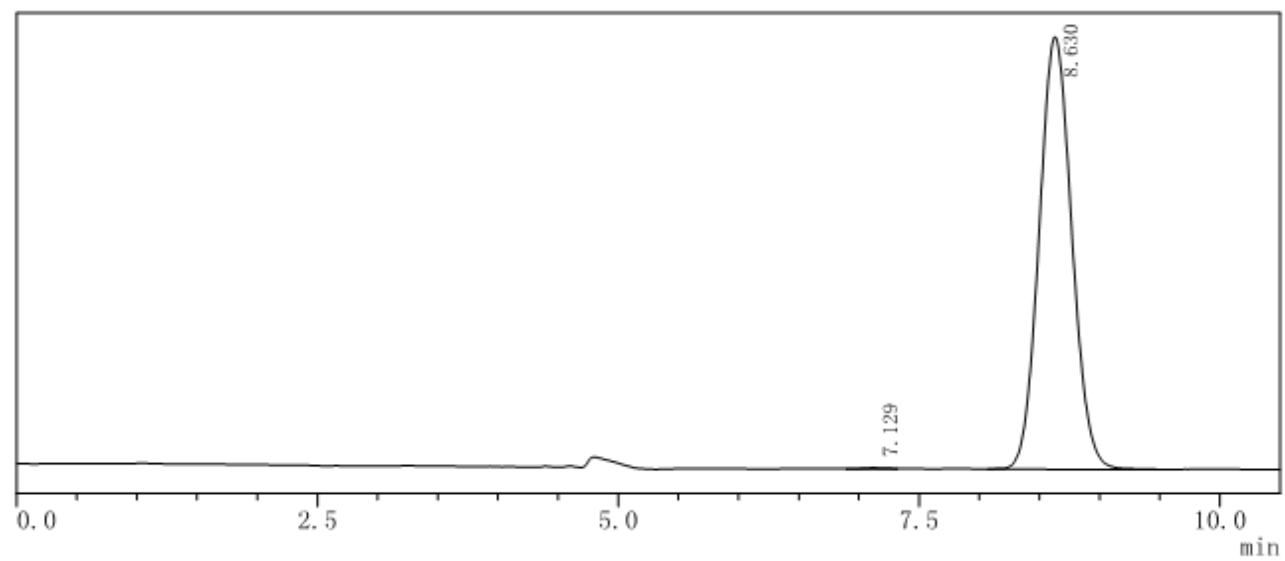

\begin{tabular}{|c|c|c|c|c|c|}
\hline \multicolumn{6}{|c|}{ 检测器 $A$ Ch1 254nm } \\
\hline Peak NO. & Retention Time & $\begin{aligned} \text { Height } & \\
& \\
& \end{aligned}$ & $\begin{array}{r}\text { Height } \% \\
0.335\end{array}$ & $\begin{array}{l}\text { Area } \\
2407\end{array}$ & $\begin{array}{c}\text { Area } \% \\
0.284\end{array}$ \\
\hline & 8.630 & 44675 & 99.665 & 845123 & 99.716 \\
\hline 总访 & & 44825 & 100.000 & 847529 & 100.000 \\
\hline
\end{tabular}



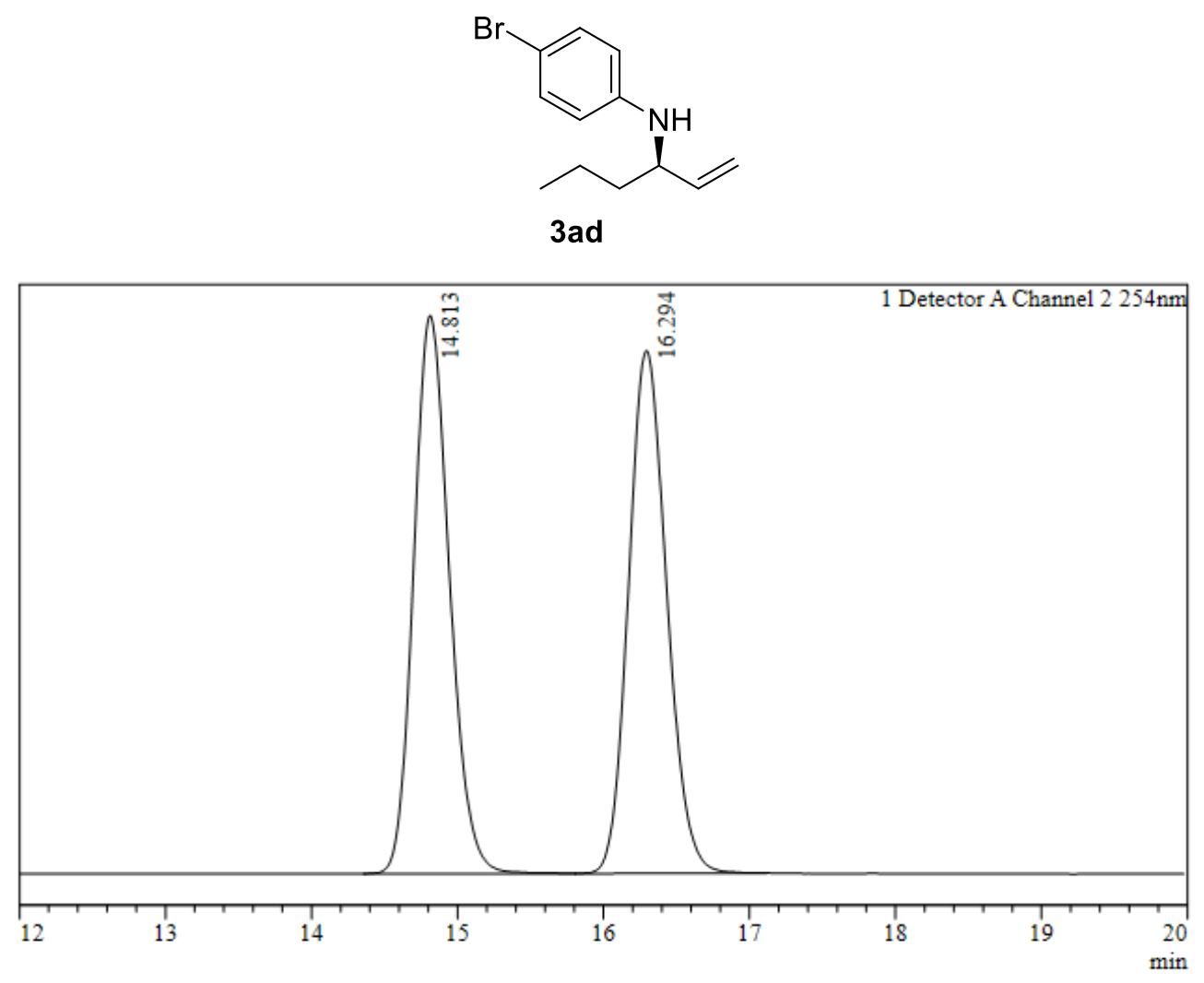

Peak Table

\begin{tabular}{|c|c|c|c|c|c|c|}
\hline Detector & 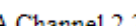 & & & & & \\
\hline Peak\# & $\begin{array}{r}\text { Ret. Time } \\
14.813\end{array}$ & $\begin{array}{l}\text { Area } \\
769218\end{array}$ & $\begin{array}{l}\text { Height } \\
45063\end{array}$ & $\begin{array}{l}\text { Area } \% \\
50.069\end{array}$ & $\begin{array}{l}\text { Conc. } \\
50.069\end{array}$ & Name \\
\hline 2 & 16.294 & 767108 & 42219 & 49.931 & 49.931 & \\
\hline Total & & 1536326 & 87281 & 100.000 & & \\
\hline
\end{tabular}

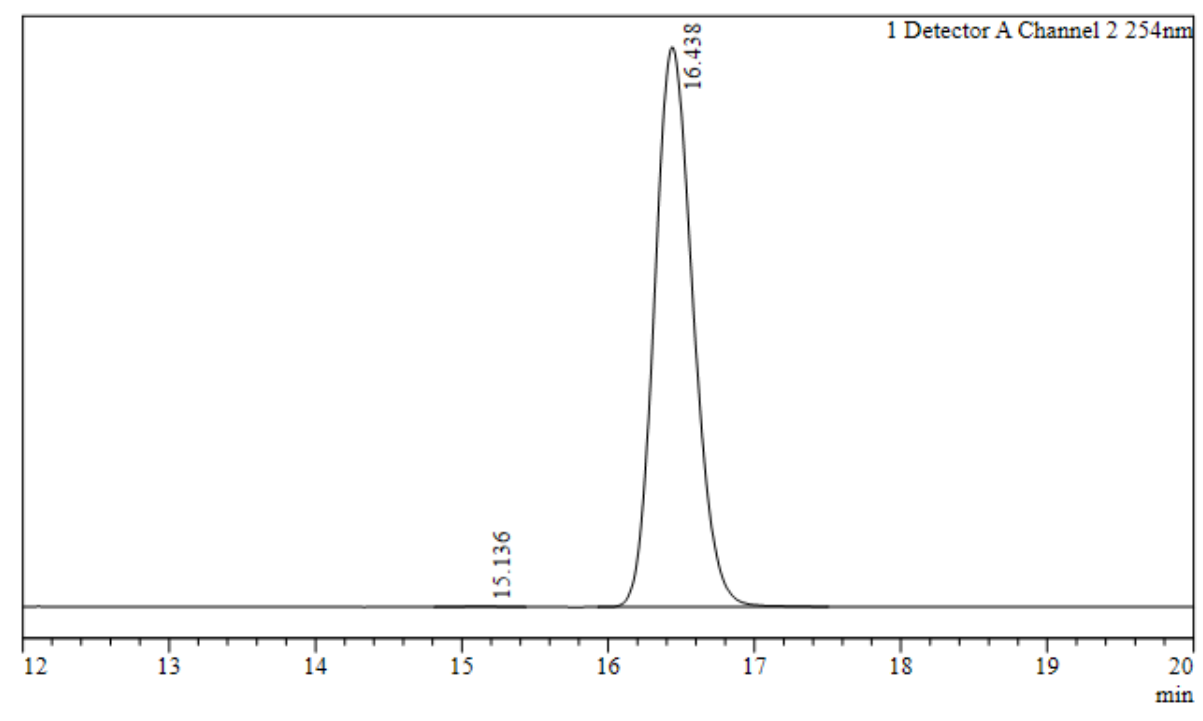

Peak Table

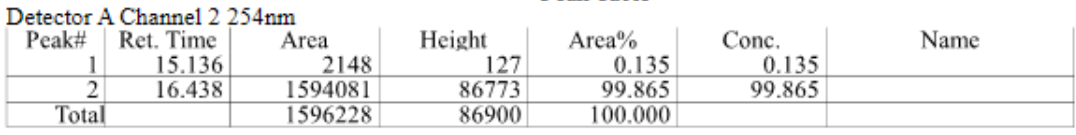




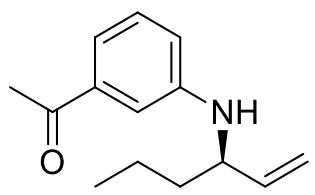

3ae

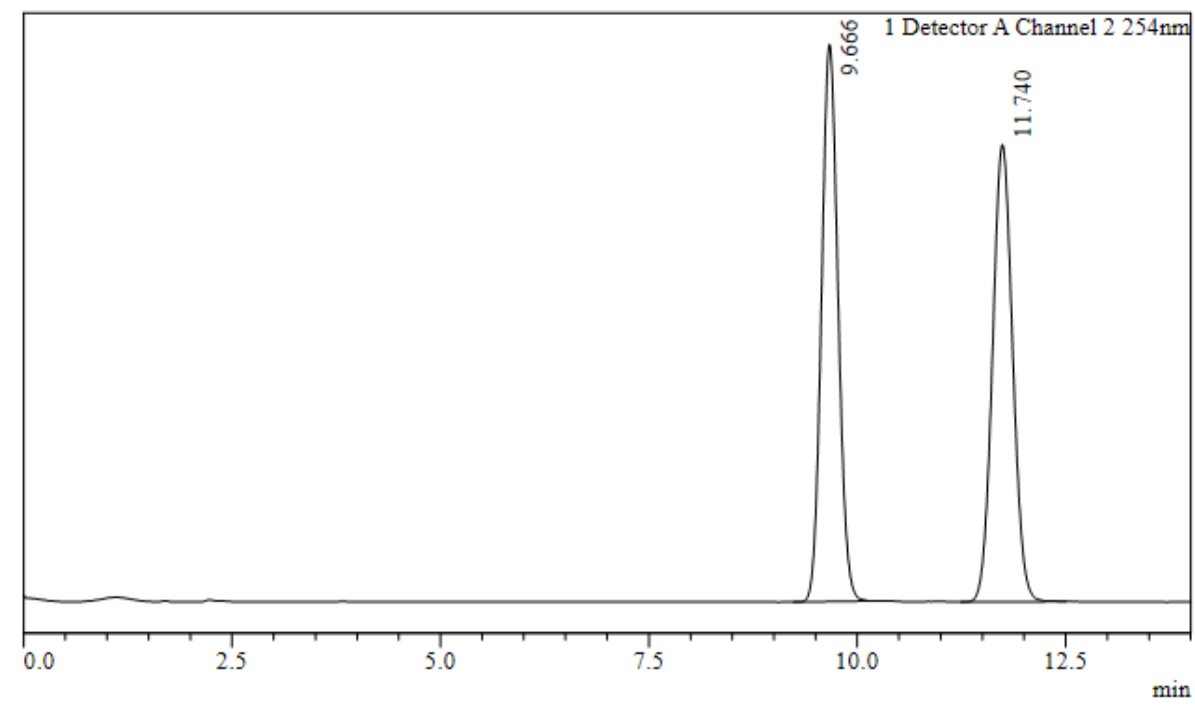

Peak Table

\begin{tabular}{|c|c|c|c|c|c|c|}
\hline Detector & A Channel 2 & & & & & \\
\hline Peak\# $_{1}$ & Ret. Time & $\begin{array}{l}\text { Area } \\
516767\end{array}$ & $\begin{array}{l}\text { Height } \\
37511\end{array}$ & $\begin{array}{l}\text { Area } \% \\
49.973\end{array}$ & $\begin{array}{l}\text { Conc. } \\
49.973\end{array}$ & Name \\
\hline 2 & 11.740 & 517326 & 30776 & 50.027 & 50.027 & \\
\hline Total & & 1034094 & 68287 & 100.000 & & \\
\hline
\end{tabular}

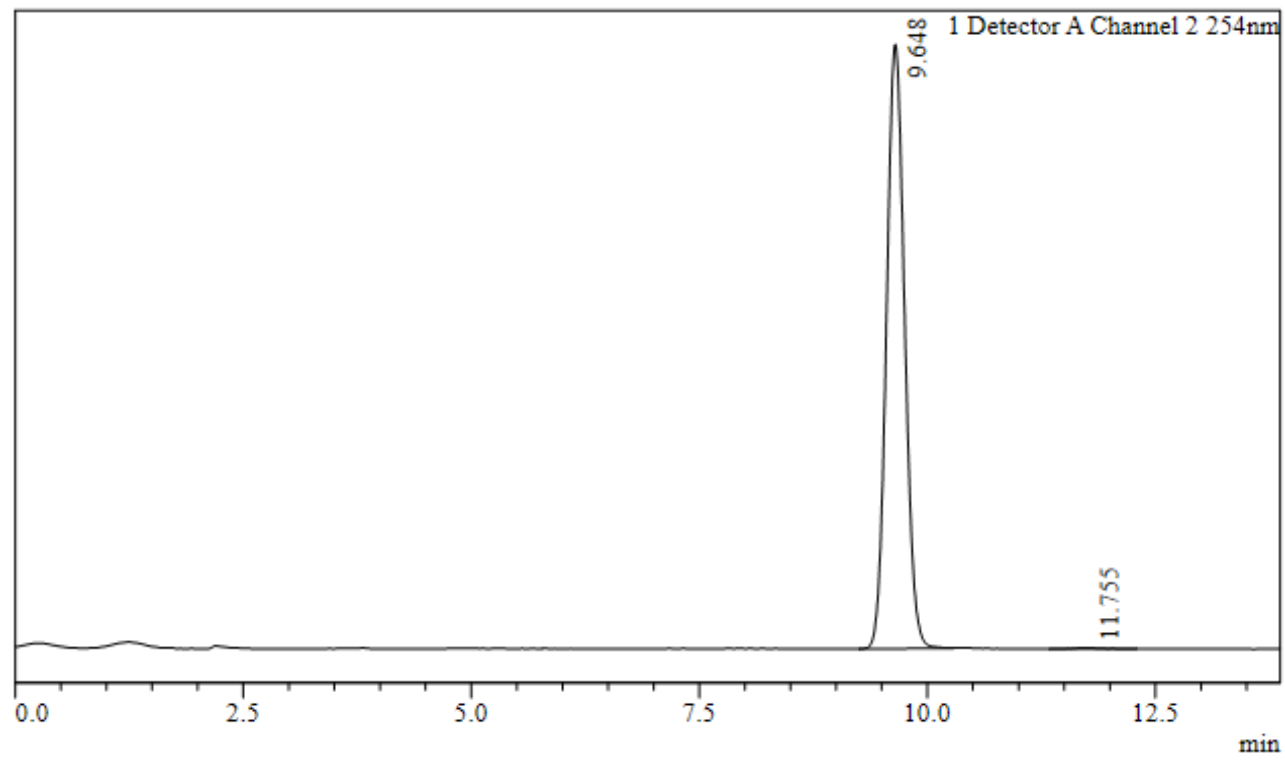

Peak Table

\begin{tabular}{|c|c|c|c|c|c|c|}
\hline \multirow{2}{*}{\multicolumn{7}{|c|}{ Detector A Channel $2254 \mathrm{~nm}$}} \\
\hline & & & & & & \\
\hline Peak\# $_{1}$ & $\begin{array}{r}\text { Ret. Time } \\
9.648\end{array}$ & $\begin{array}{l}\text { Area } \\
383506\end{array}$ & $\begin{array}{l}\text { Height } \\
27722\end{array}$ & $\begin{array}{l}\text { Area } \% \\
99.759\end{array}$ & $\begin{array}{l}\text { Conc. } \\
99.759\end{array}$ & Name \\
\hline 2 & 11.755 & 926 & 46 & 0.241 & 0.241 & \\
\hline Total & & 384432 & 27767 & 100.000 & & \\
\hline
\end{tabular}



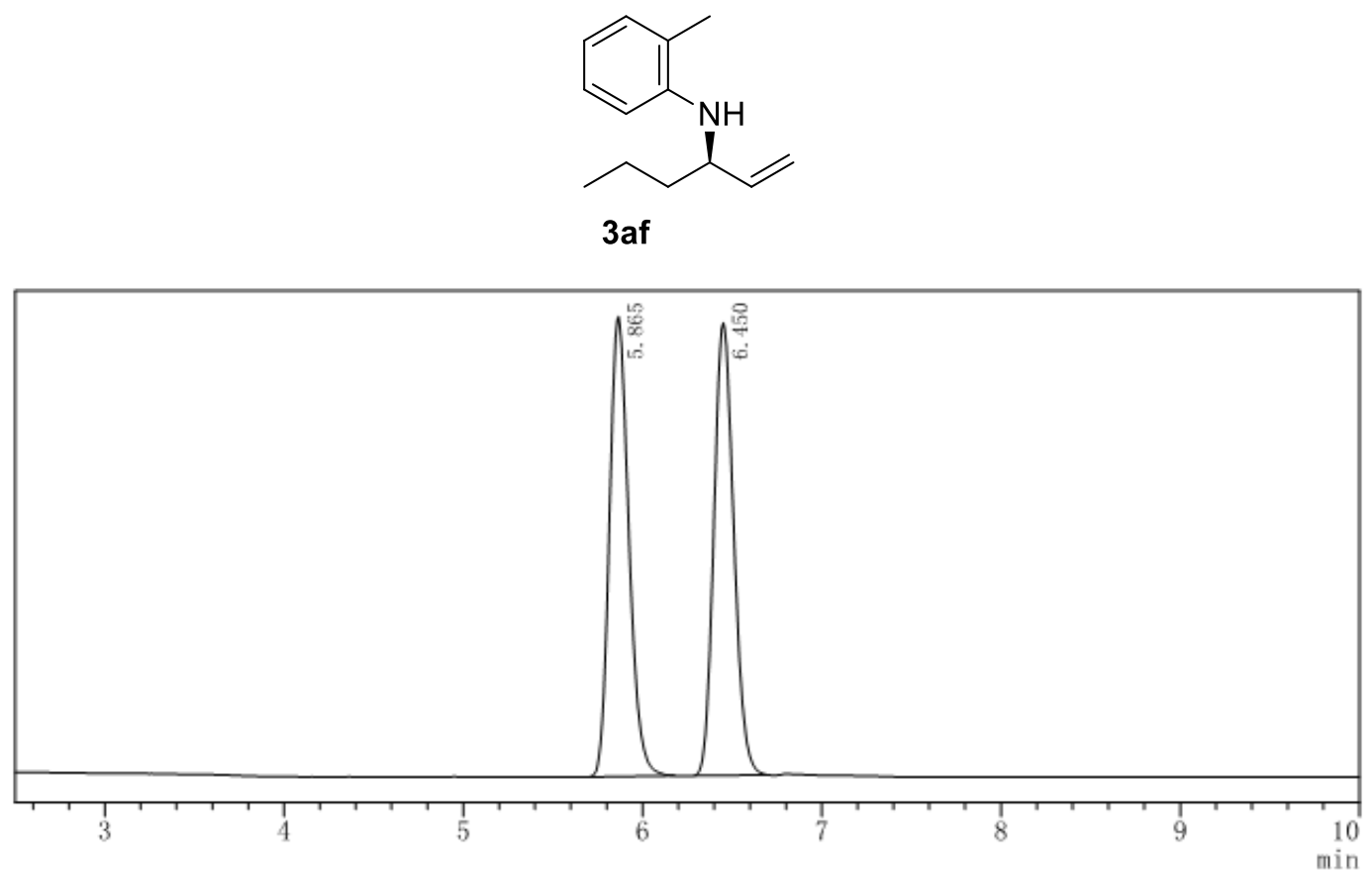

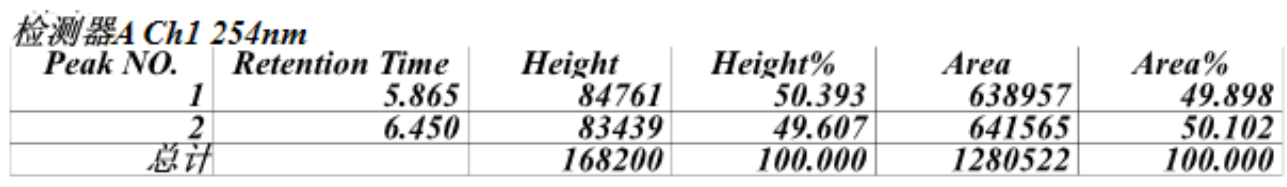

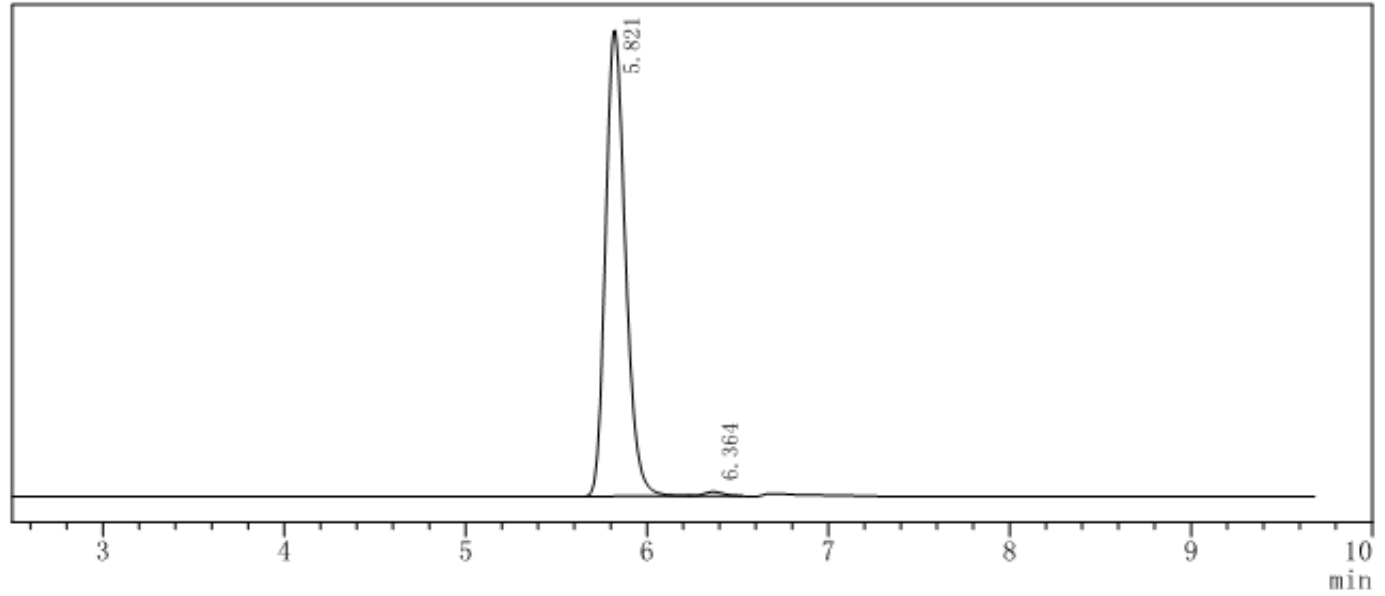

检测器A Ch1 254nm

\begin{tabular}{|r|r|r|r|r|r|}
\hline Peak NO. & Retention Time & Height & Height\% & \multicolumn{1}{|c|}{ Area } & \multicolumn{1}{|c|}{ Area\% } \\
1 & 5.821 & 101347 & 99.115 & 774854 & 99.155 \\
\hline 2 & 6.364 & 905 & 0.885 & 6603 & 0.845 \\
\hline 总计 & & 102251 & 100.000 & 781457 & 100.000 \\
\hline
\end{tabular}




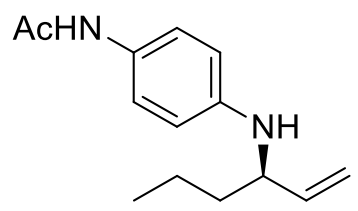

3ag

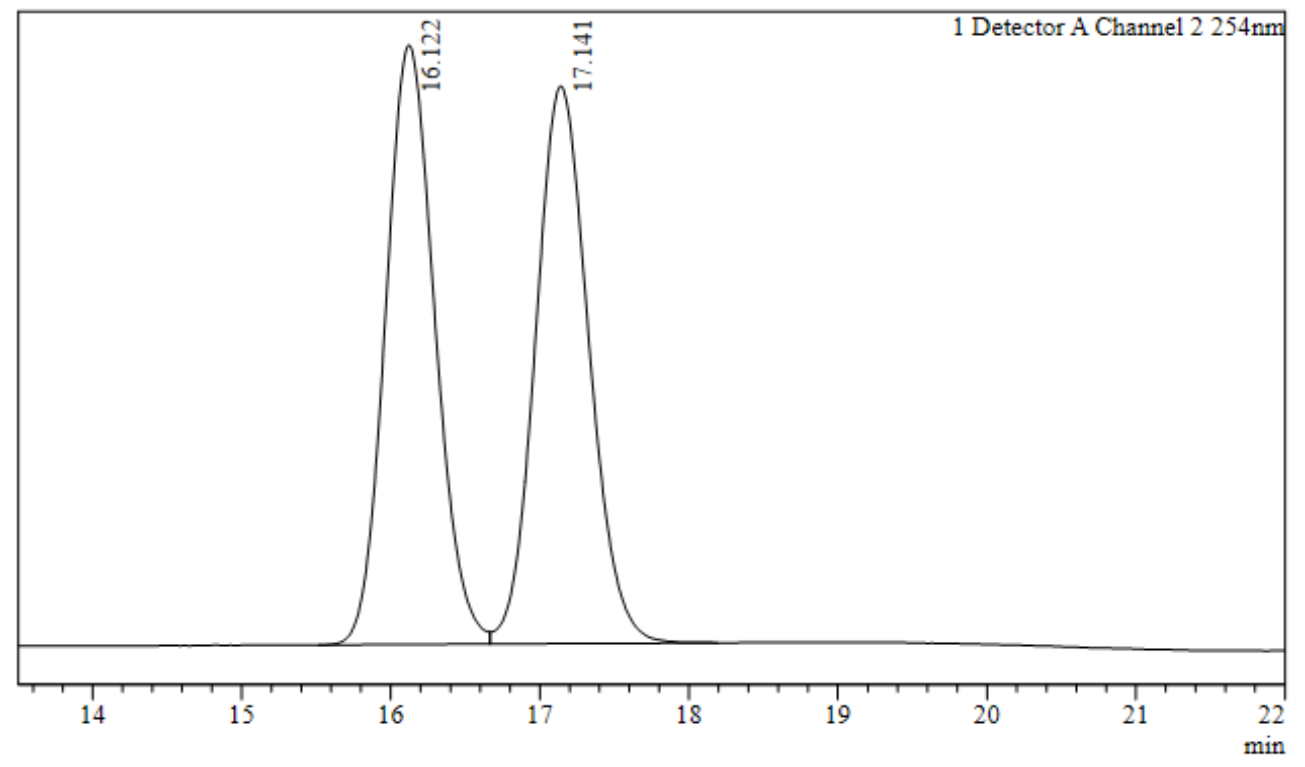

Peak Table
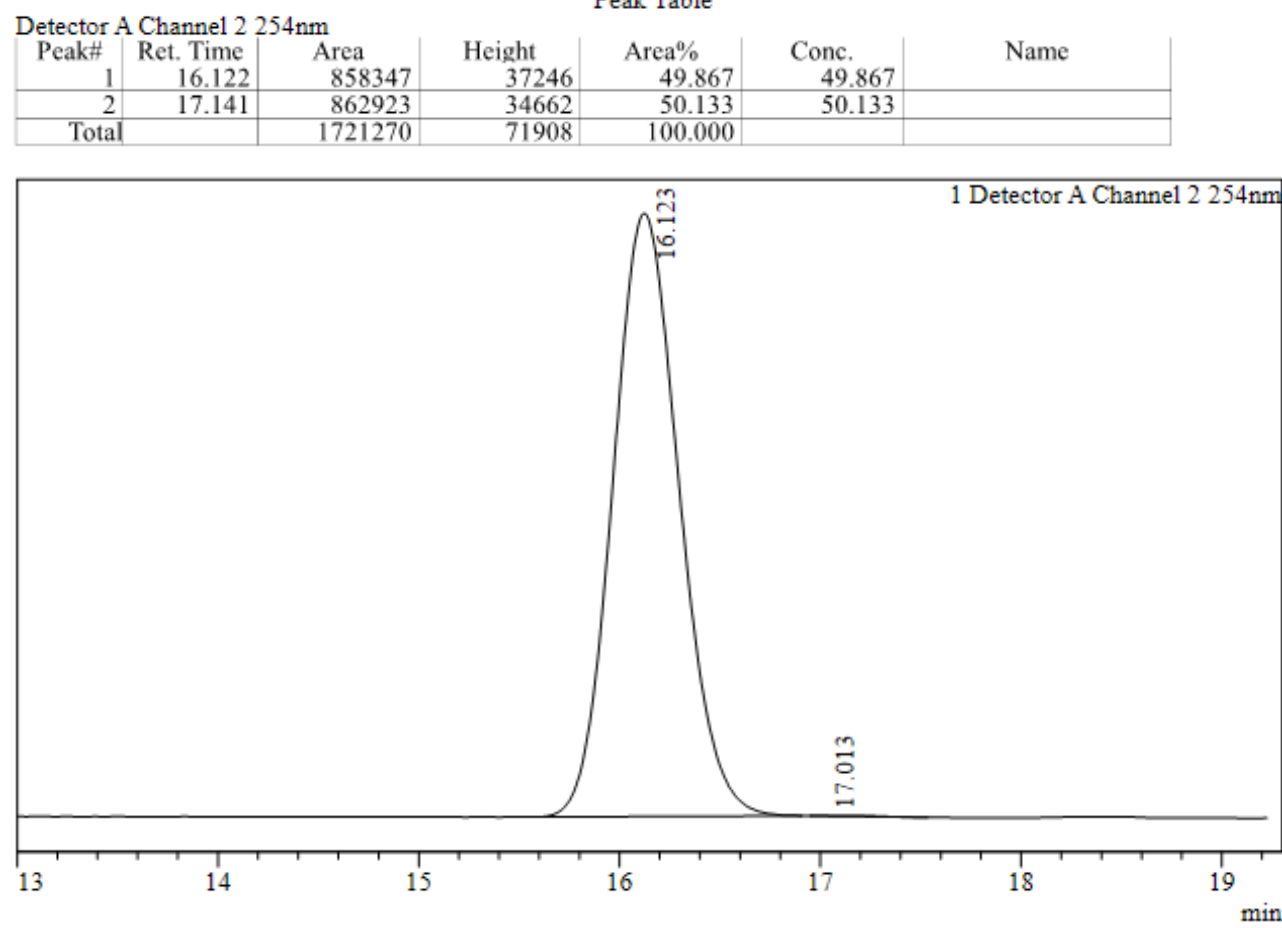

Peak Table

\begin{tabular}{|c|c|c|c|c|c|c|}
\hline \multicolumn{7}{|c|}{ Detector A Channel $2254 \mathrm{~nm}$} \\
\hline $\begin{array}{r}\text { Peak\# } \\
1 \\
\end{array}$ & $\begin{array}{r}\text { Ret. Time } \\
16.123\end{array}$ & $\begin{array}{l}\text { Area } \\
1034513\end{array}$ & $\begin{array}{l}\text { Height } \\
\quad 45007\end{array}$ & $\begin{array}{l}\text { Area } \% \\
99.917\end{array}$ & $\begin{array}{l}\text { Conc. } \\
99.917\end{array}$ & Name \\
\hline 2 & 17.013 & 856 & 25 & 0.083 & 0.083 & \\
\hline Total & & 1035369 & 45032 & 100.000 & & \\
\hline
\end{tabular}




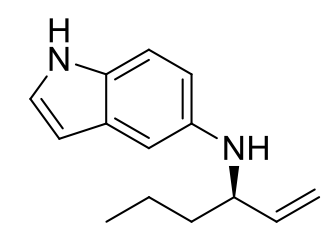

3ah

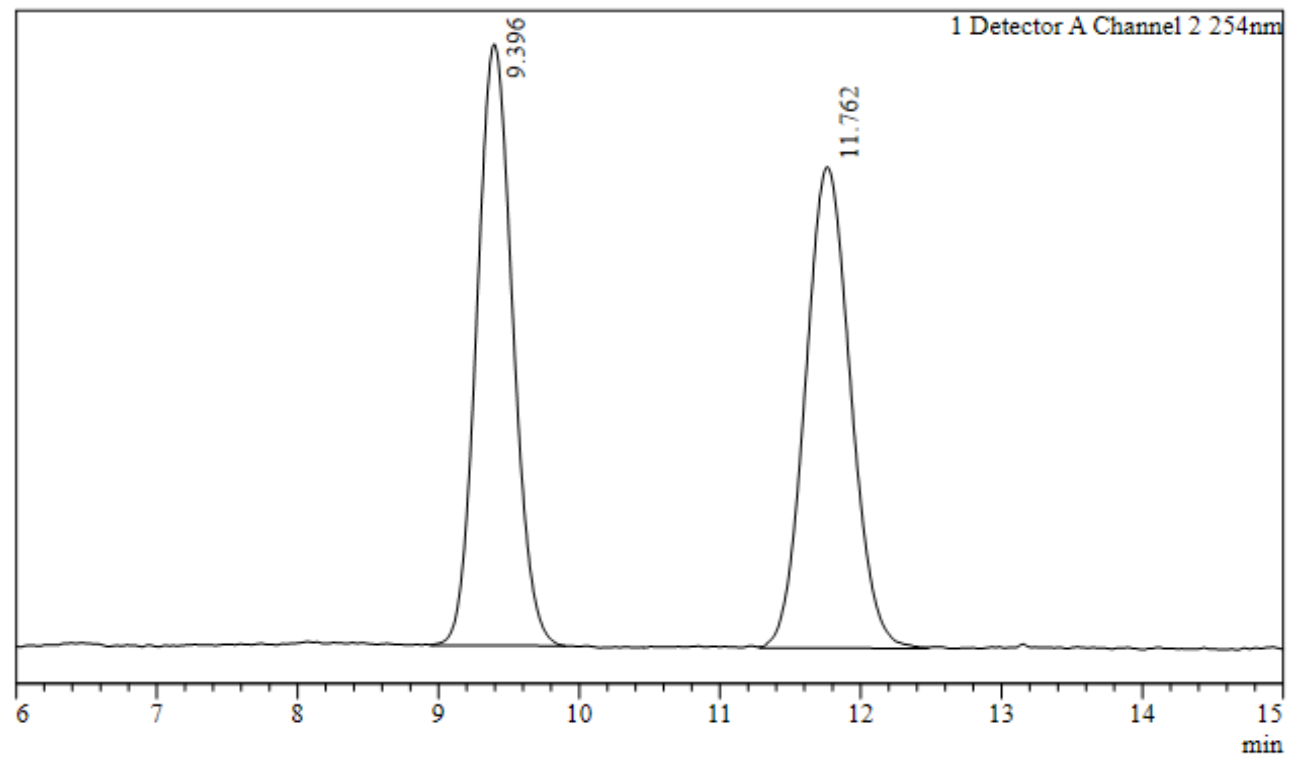

Peak Table
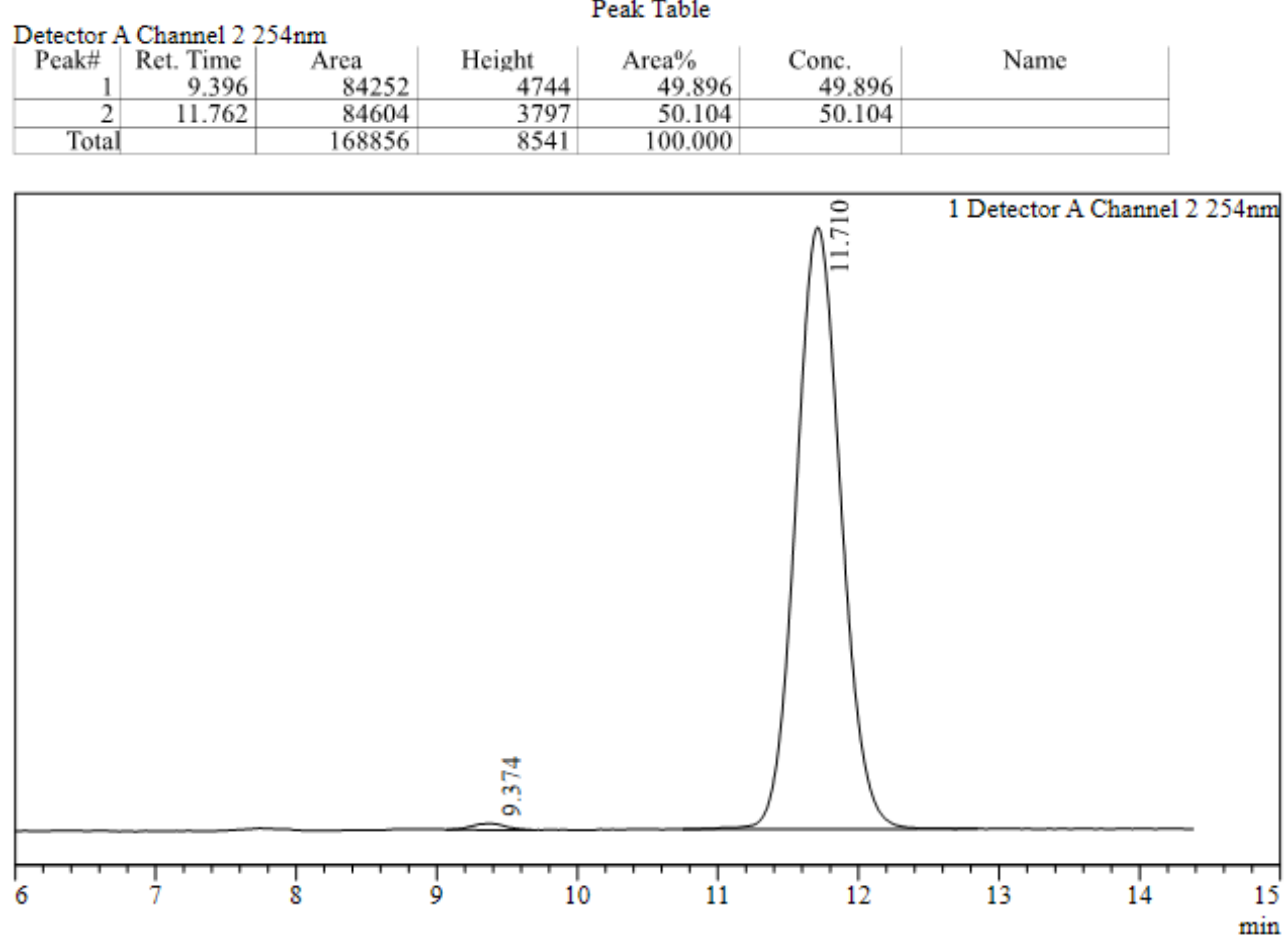

Peak Table

\begin{tabular}{|c|c|c|c|c|c|c|}
\hline \multicolumn{7}{|c|}{ Detector A Channel $2254 \mathrm{~nm}$} \\
\hline${ }_{1}$ Peak\# & $\begin{array}{r}\text { Ret. Time } \\
9.374\end{array}$ & $\begin{array}{l}\text { Area } \\
2395\end{array}$ & $\begin{array}{l}\text { Height } \\
\\
144\end{array}$ & $\begin{array}{c}\text { Area } \% \\
0.778\end{array}$ & $\begin{array}{l}\text { Conc. } \\
0.778\end{array}$ & Name \\
\hline 2 & 11.710 & 305359 & 13682 & 99.222 & 99.222 & \\
\hline Tota & & 307754 & 13826 & 100.000 & & \\
\hline
\end{tabular}




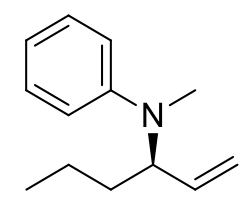

3ai

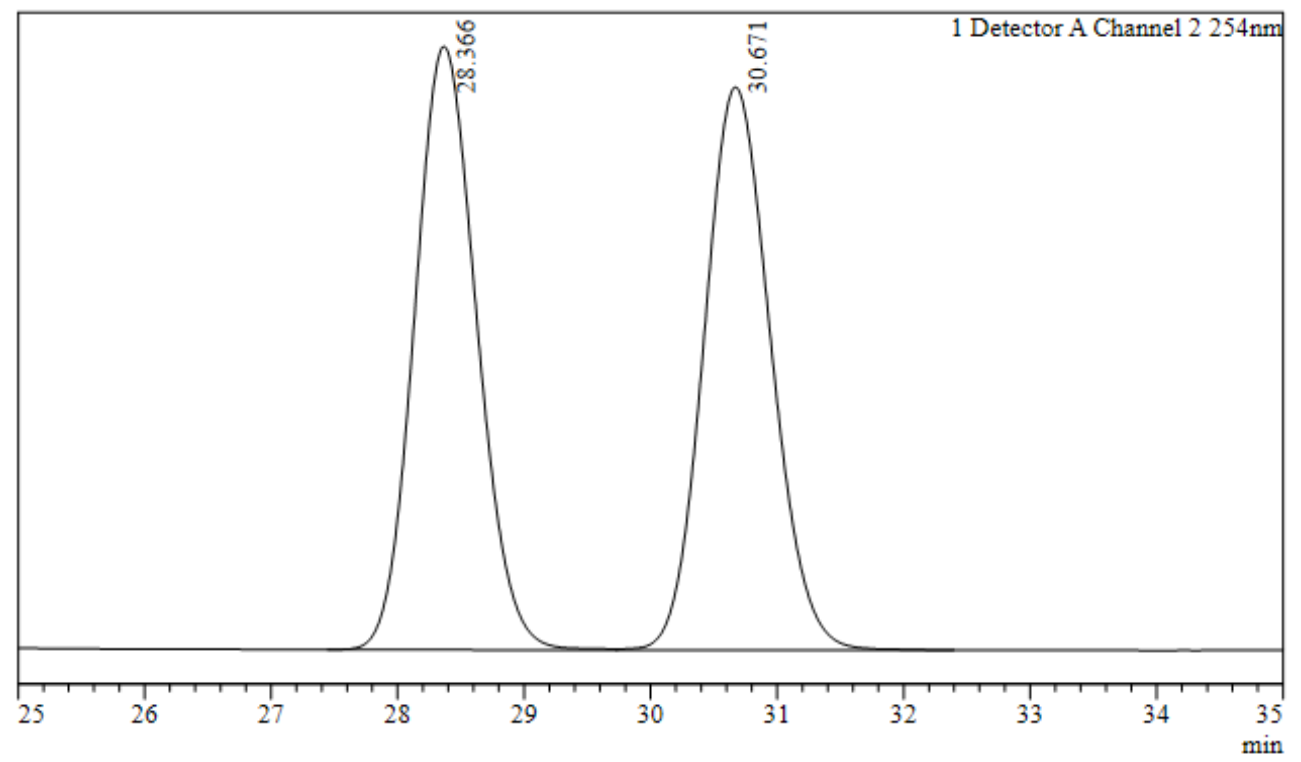

Peak Table
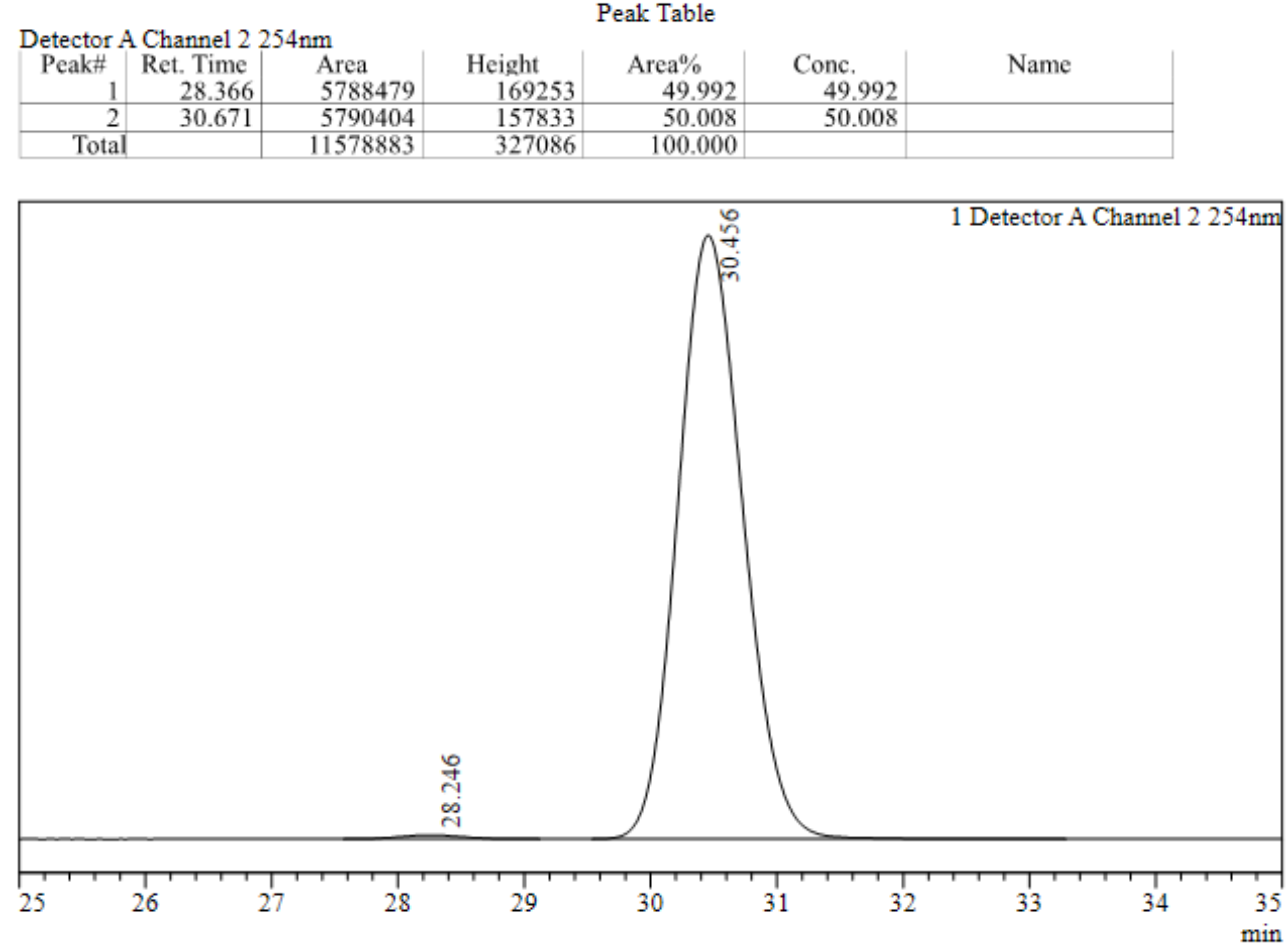

Peak Table

\begin{tabular}{|c|c|c|c|c|c|c|}
\hline \multicolumn{7}{|c|}{ Detector A Channel $2254 \mathrm{~nm}$} \\
\hline Peak\# & $\begin{array}{r}\text { Ret. Time } \\
28.246\end{array}$ & $\begin{array}{l}\text { Area } \\
\quad 46021\end{array}$ & $\begin{array}{l}\text { Height } \\
1391\end{array}$ & $\begin{array}{l}\text { Area } \% \\
0.602\end{array}$ & $\begin{array}{l}\text { Conc. } \\
0.602\end{array}$ & Name \\
\hline 2 & 30.456 & 7592389 & 212334 & 99.398 & 99.398 & \\
\hline Tota & & 7638411 & 213725 & 100.000 & & \\
\hline
\end{tabular}




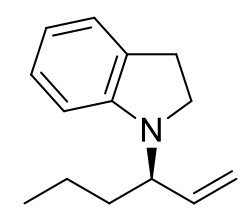

3aj

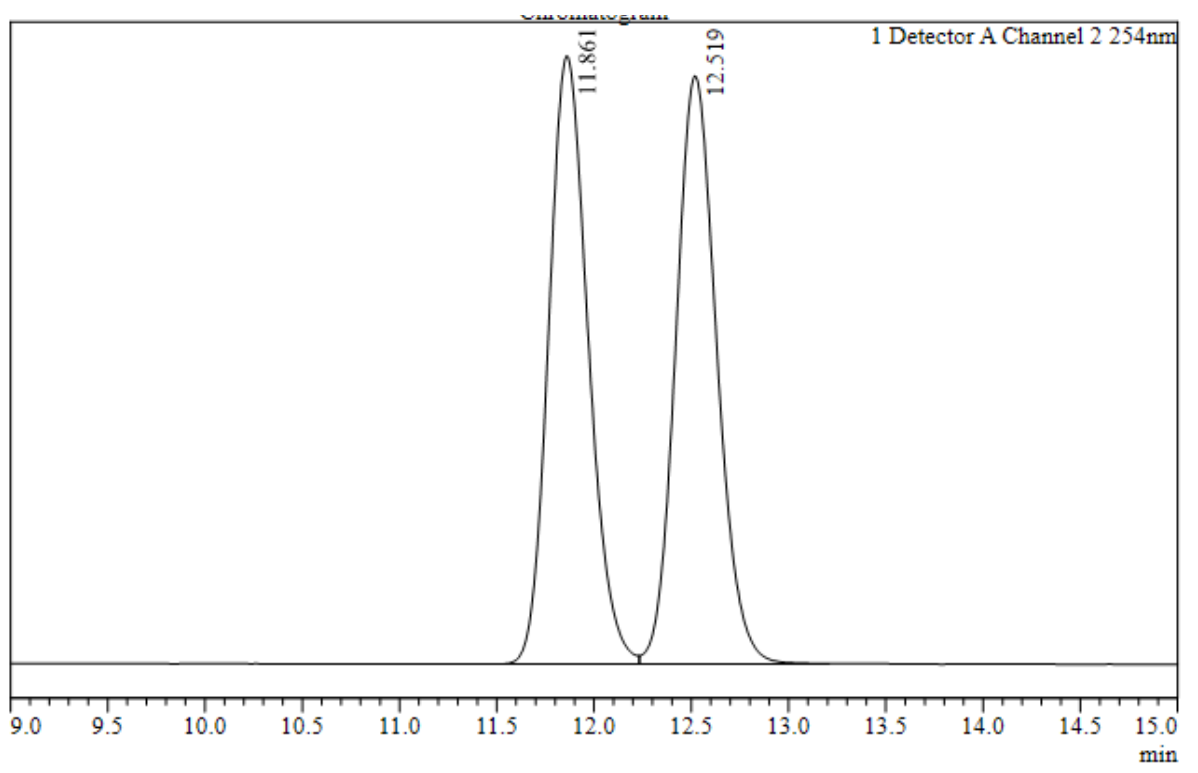

Peak Table
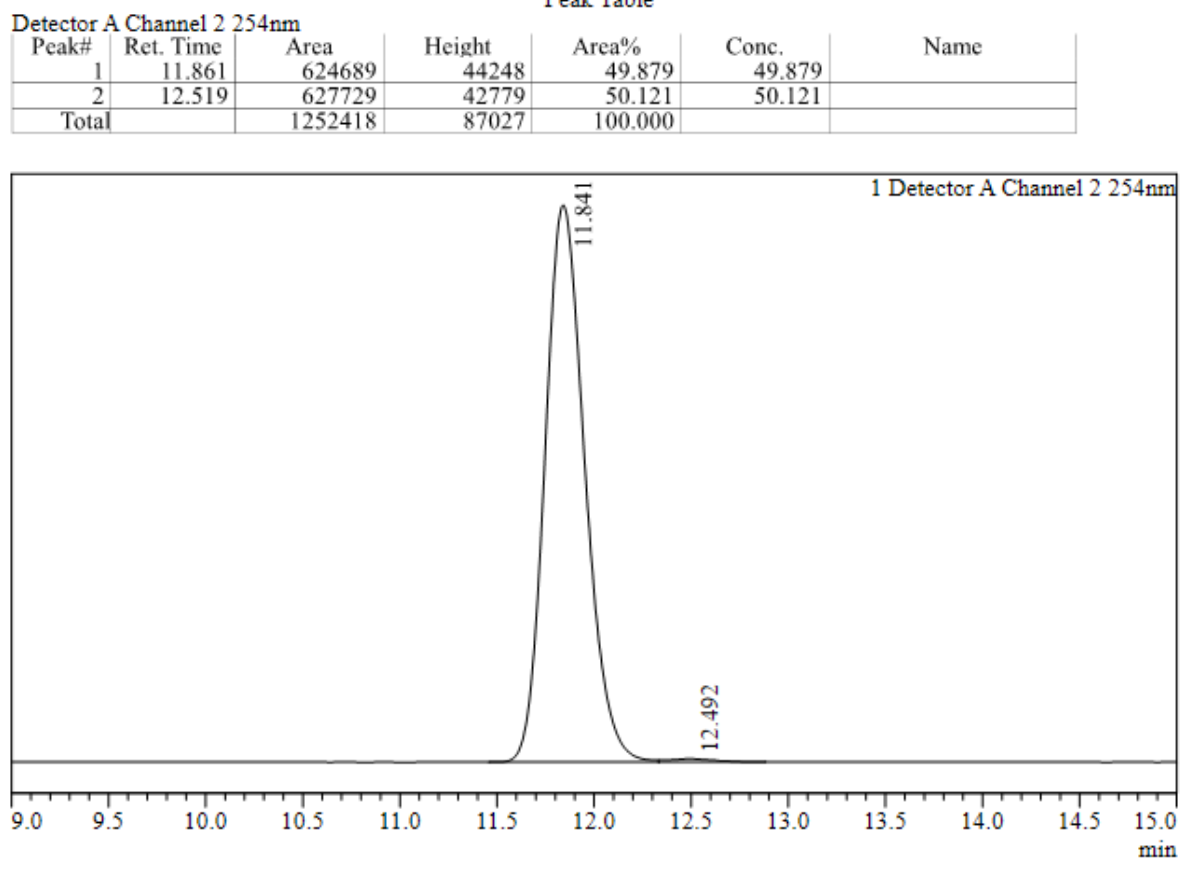

Peak Table

\begin{tabular}{|c|c|c|c|c|c|c|}
\hline \multicolumn{7}{|c|}{ Detector A Channel $2254 \mathrm{~nm}$} \\
\hline Peak\# & Ret. Time & Area & $\begin{array}{l}\text { Height } \\
75207\end{array}$ & $\begin{array}{c}\text { Area } \% \\
99378\end{array}$ & $\begin{array}{l}\text { Conc. } \\
99.378\end{array}$ & Name \\
\hline 2 & 12.492 & 6705 & 428 & 0.622 & 0.622 & \\
\hline Total & & 1078159 & 75634 & 100.000 & & \\
\hline
\end{tabular}




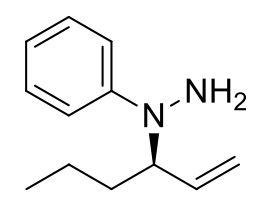

3ak
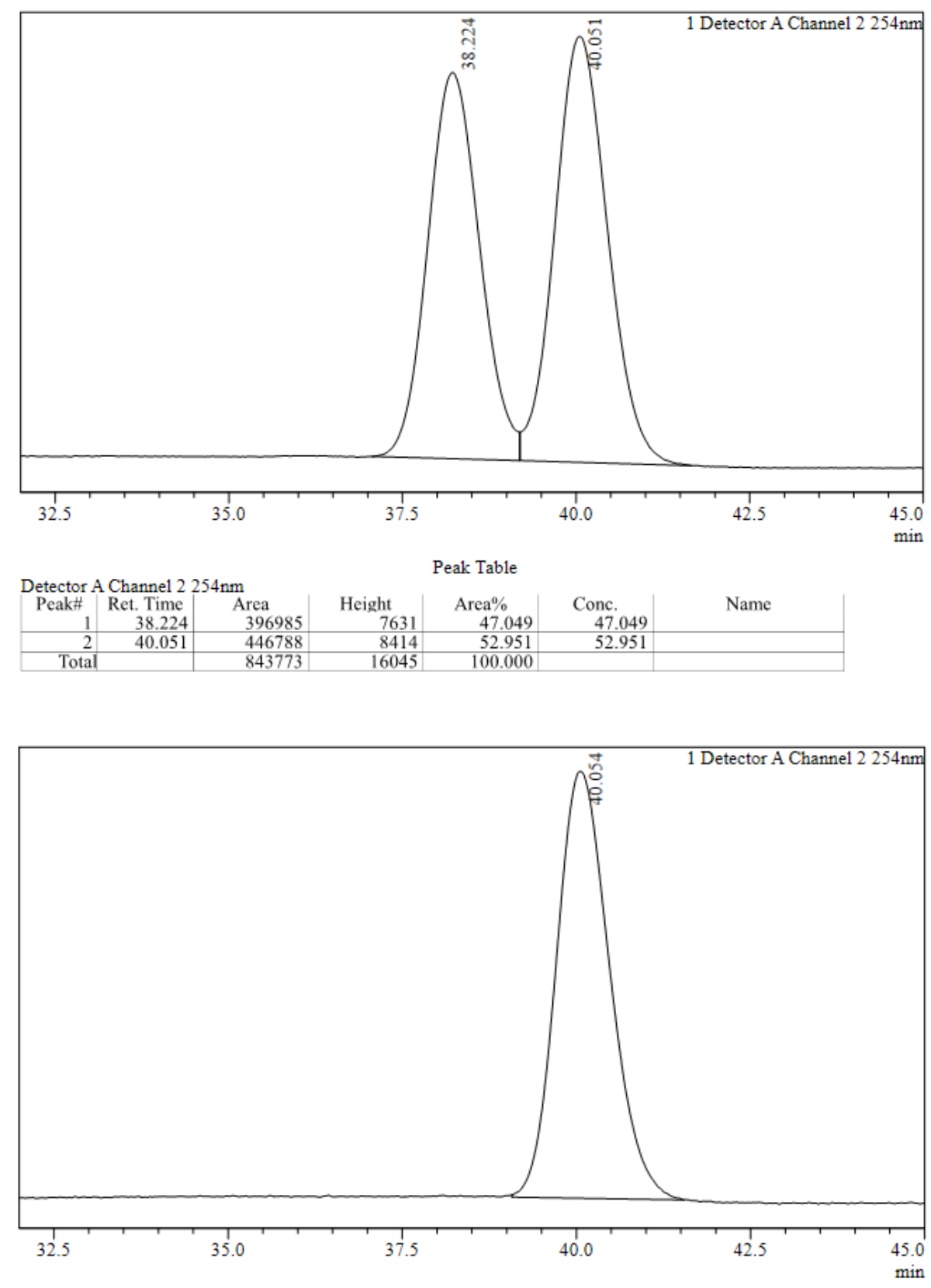

\begin{tabular}{|c|c|c|c|c|c|c|}
\hline \multirow{2}{*}{\multicolumn{7}{|c|}{ Detector A Channel $2254 \mathrm{~nm}$}} \\
\hline & & & & & & \\
\hline $\begin{array}{r}\text { Peak\# } \\
1\end{array}$ & $\begin{array}{r}\text { Ret. Time } \\
40.054\end{array}$ & $\begin{array}{l}\text { Area } \\
236821\end{array}$ & $\begin{array}{l}\text { Height } \\
4463\end{array}$ & $\begin{array}{l}\text { Area } \% \\
\quad 100.000\end{array}$ & $\begin{array}{l}\text { Conc. } \\
100.000\end{array}$ & Name \\
\hline Total & & 236821 & 4463 & 100.000 & & \\
\hline
\end{tabular}



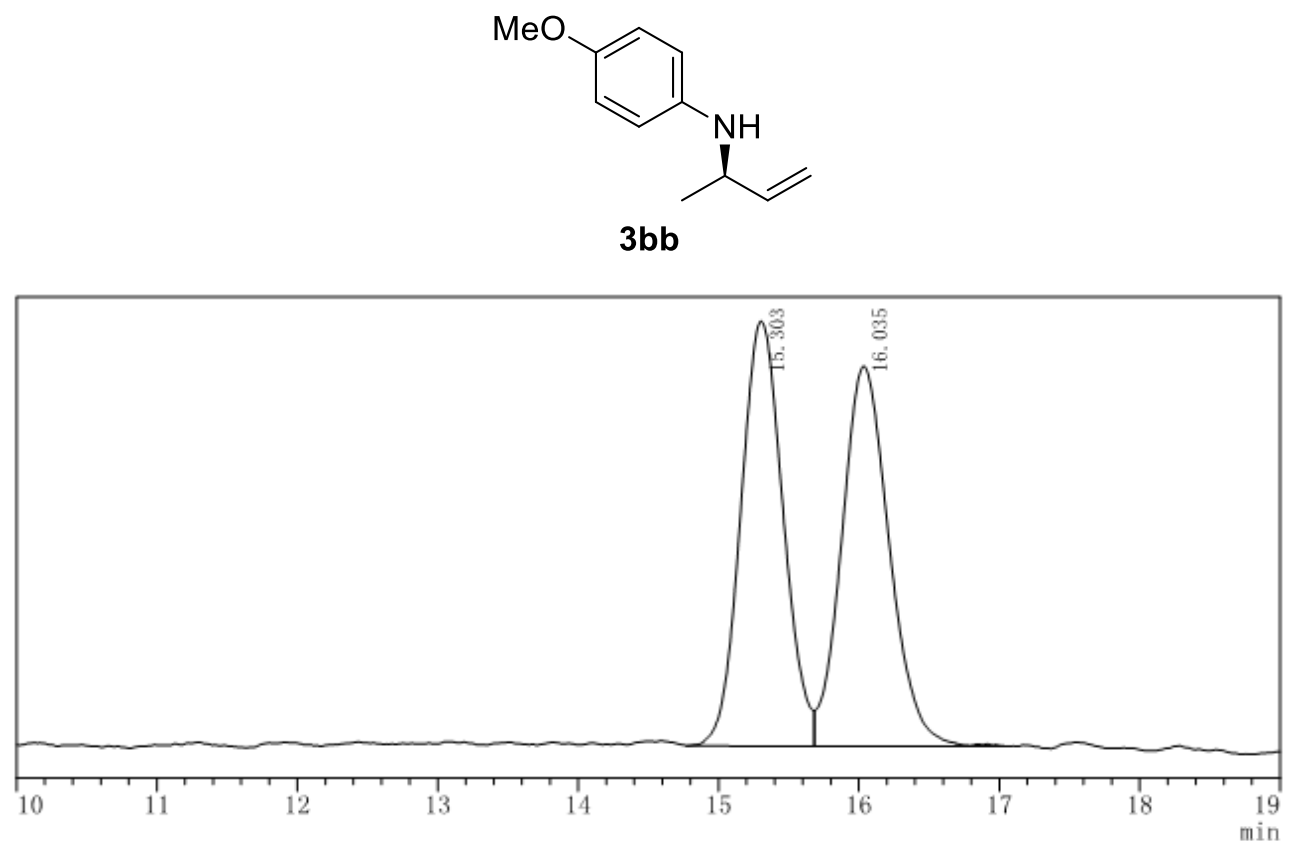

\begin{tabular}{|c|c|c|c|c|c|}
\hline Peak NO. & $\begin{array}{r}\text { Retention Time } \\
15.303\end{array}$ & $\begin{array}{l}\text { Height } \\
5298\end{array}$ & $\begin{array}{l}\text { Height } \% \\
52.770\end{array}$ & $\underset{111413}{\text { Area }}$ & $\begin{array}{l}\text { Area } \% \\
\quad 49.984\end{array}$ \\
\hline 2 & 16.035 & 4742 & 47.230 & 111484 & 50.016 \\
\hline 总计 & & 10040 & 100.000 & 222897 & 100.000 \\
\hline
\end{tabular}

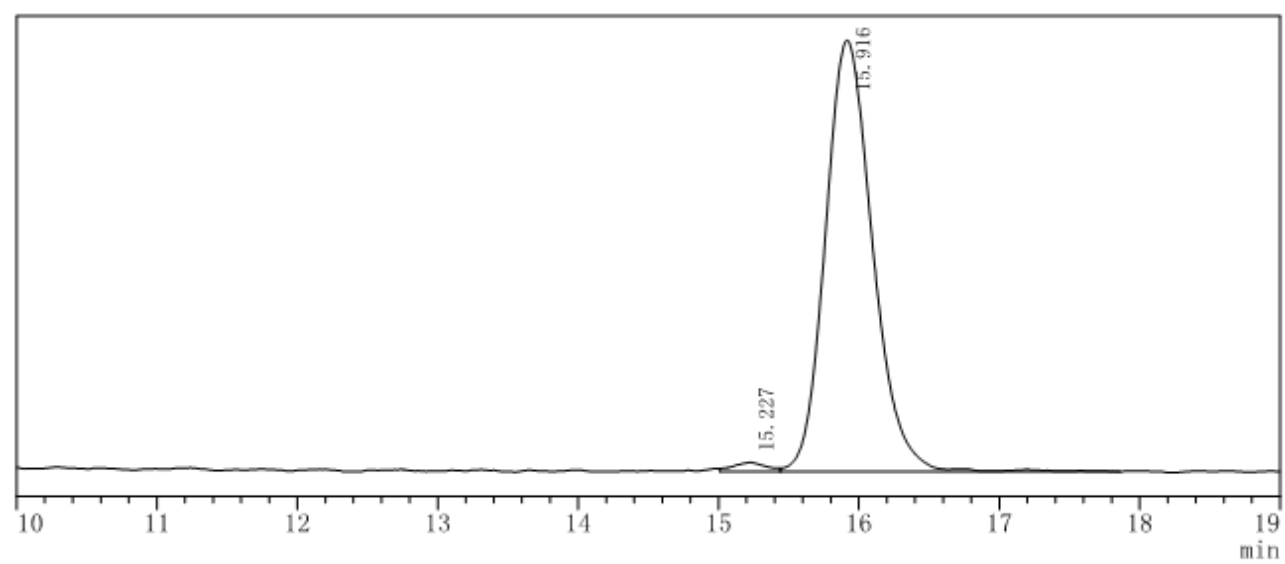

\begin{tabular}{|c|c|c|c|c|c|}
\hline Peak NO. & $\begin{array}{r}\text { Retention Time } \\
15.227\end{array}$ & ${ }^{\text {Height }_{252}}$ & $\begin{array}{c}\text { Height } \% \\
2.101\end{array}$ & ${ }_{4132}$ & $\begin{array}{l}\text { Area\% } \\
\quad 1.484\end{array}$ \\
\hline 2 & 15.916 & 11726 & 97.899 & 274342 & 98.516 \\
\hline 总计 & & 11978 & 100.000 & 278473 & 100.000 \\
\hline
\end{tabular}




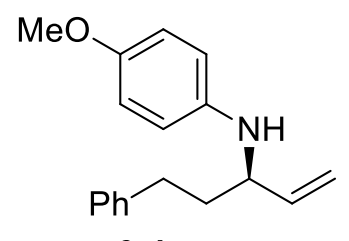

$3 \mathrm{cb}$

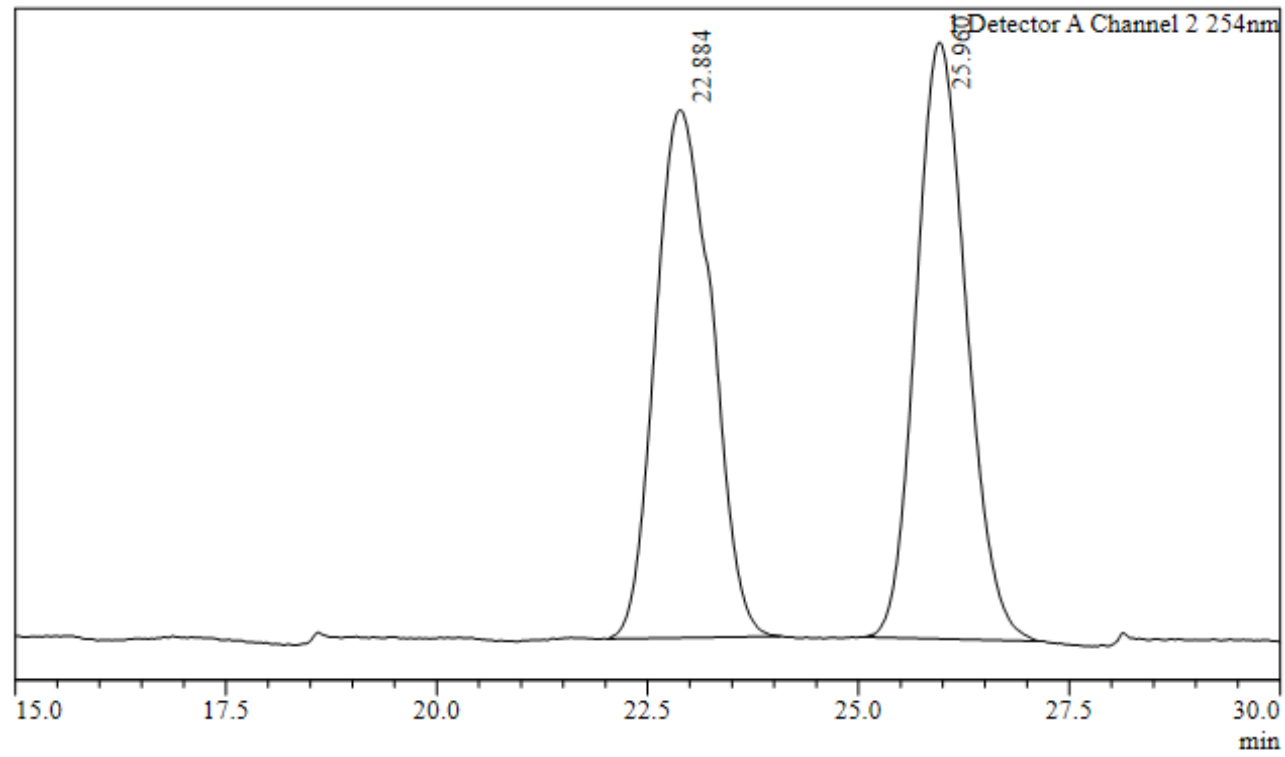

Peak Table
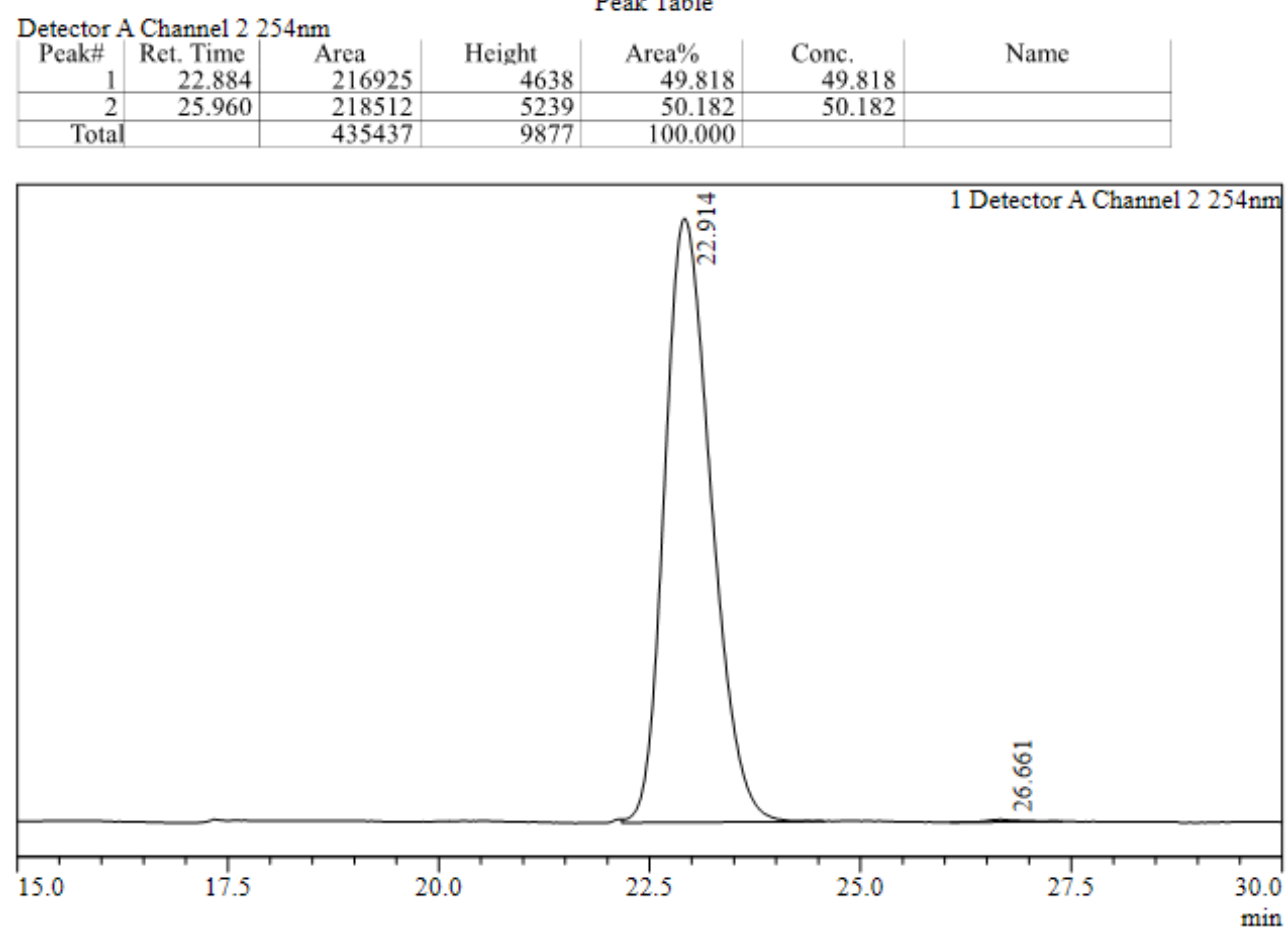

Peak Table

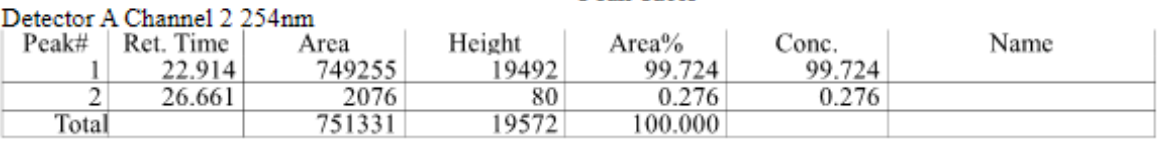




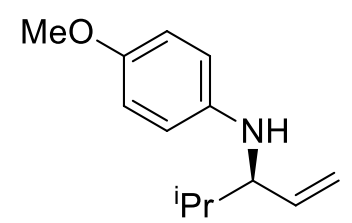

$3 \mathrm{db}$

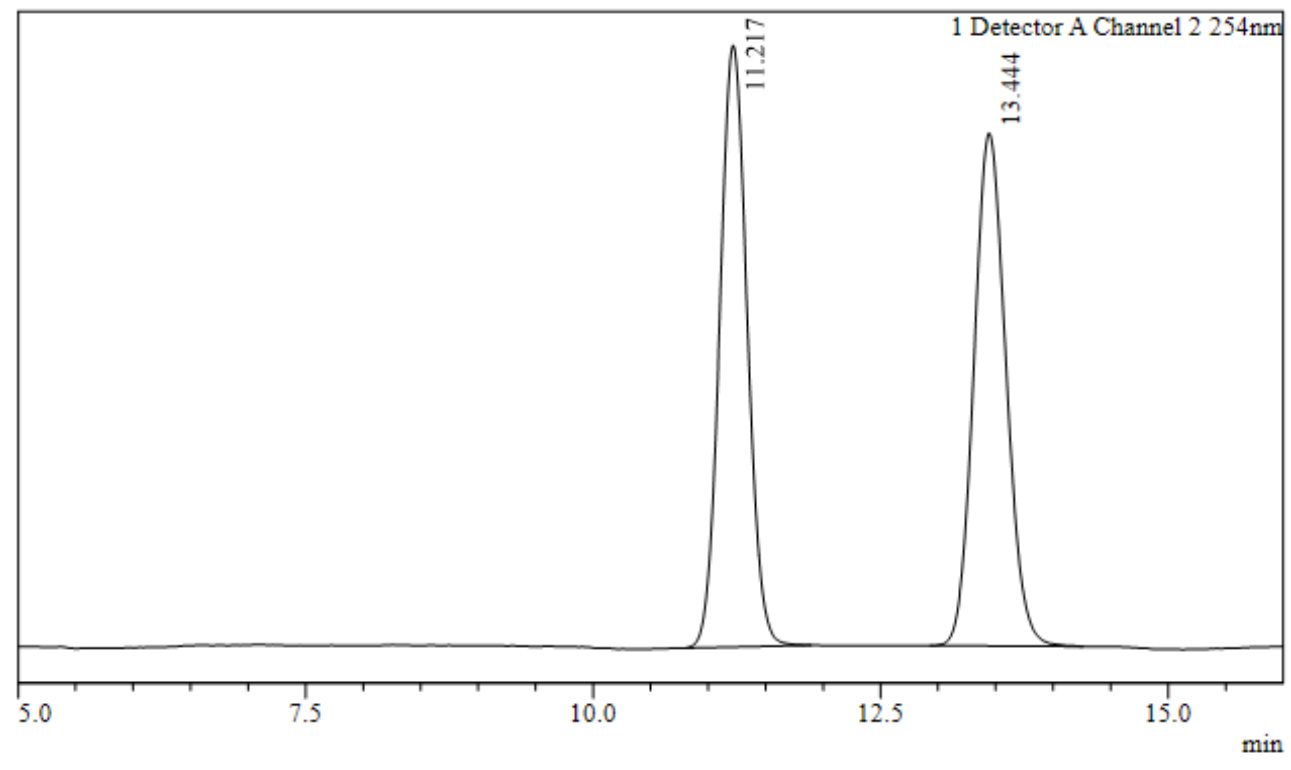

Peak Table

\begin{tabular}{|c|c|c|c|c|c|c|}
\hline \multicolumn{7}{|c|}{ Detector A Channel $2254 \mathrm{~nm}$} \\
\hline Peak\# & Ret. Time & Area & Height & $\begin{array}{l}\text { Area } \% \\
50.079\end{array}$ & $\begin{array}{l}\text { Conc. } \\
50.079\end{array}$ & Name \\
\hline 2 & 13.444 & 352136 & 18115 & 49.921 & 49.921 & \\
\hline Total & & 705381 & 39374 & 100.000 & & \\
\hline
\end{tabular}

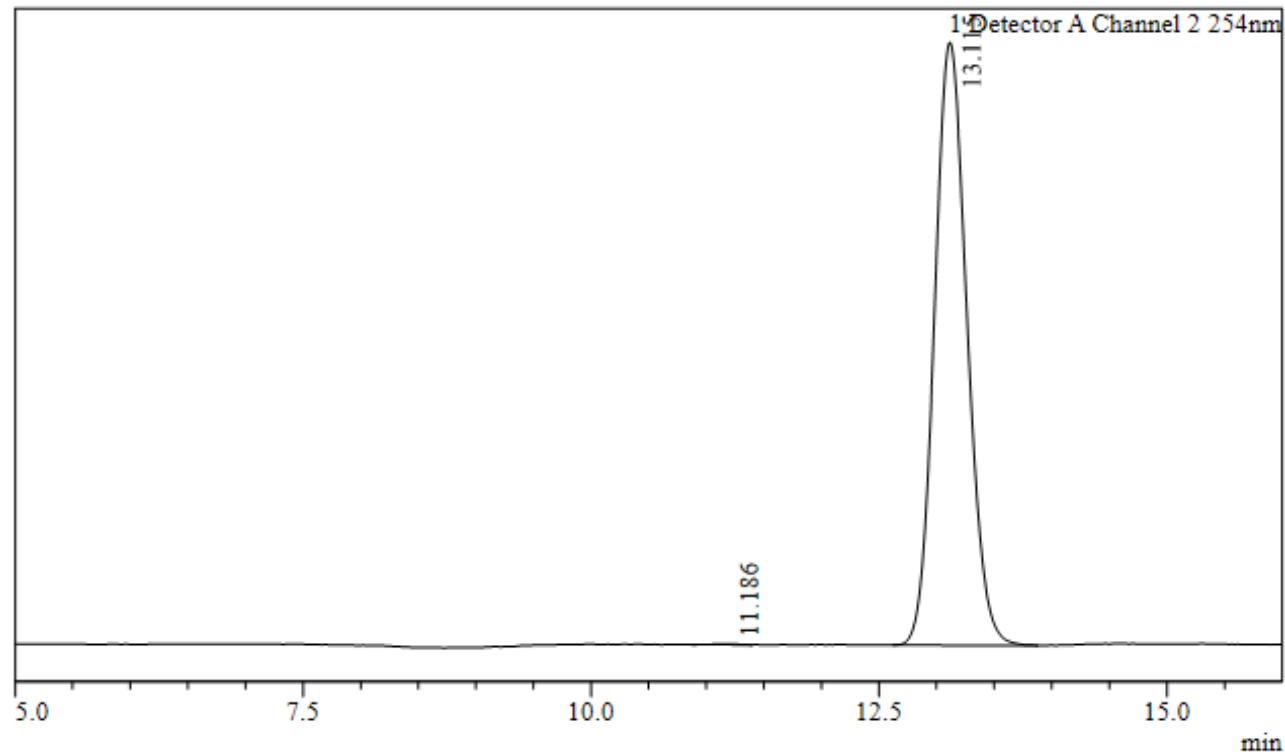

Peak Table

\begin{tabular}{|c|c|c|c|c|c|c|}
\hline Detector $A$ & 1 Channel 2 & $\mathrm{~nm}$ & & & & \\
\hline $\begin{array}{r}\text { Peak\# } \\
1\end{array}$ & $\begin{array}{r}\text { Ret. Time } \\
11.186\end{array}$ & ${ }^{\text {Area }}{ }_{407}$ & $\begin{array}{l}\text { Height } \\
\\
\end{array}$ & $\begin{array}{l}\text { Area } \% \\
0.090\end{array}$ & $\begin{array}{l}\text { Conc. } \\
0.090\end{array}$ & Name \\
\hline 2 & 13.115 & 450727 & 22984 & 99.910 & 99.910 & \\
\hline Total & & 451134 & 23019 & 100.000 & & \\
\hline
\end{tabular}




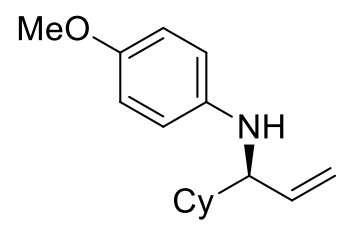

3eb

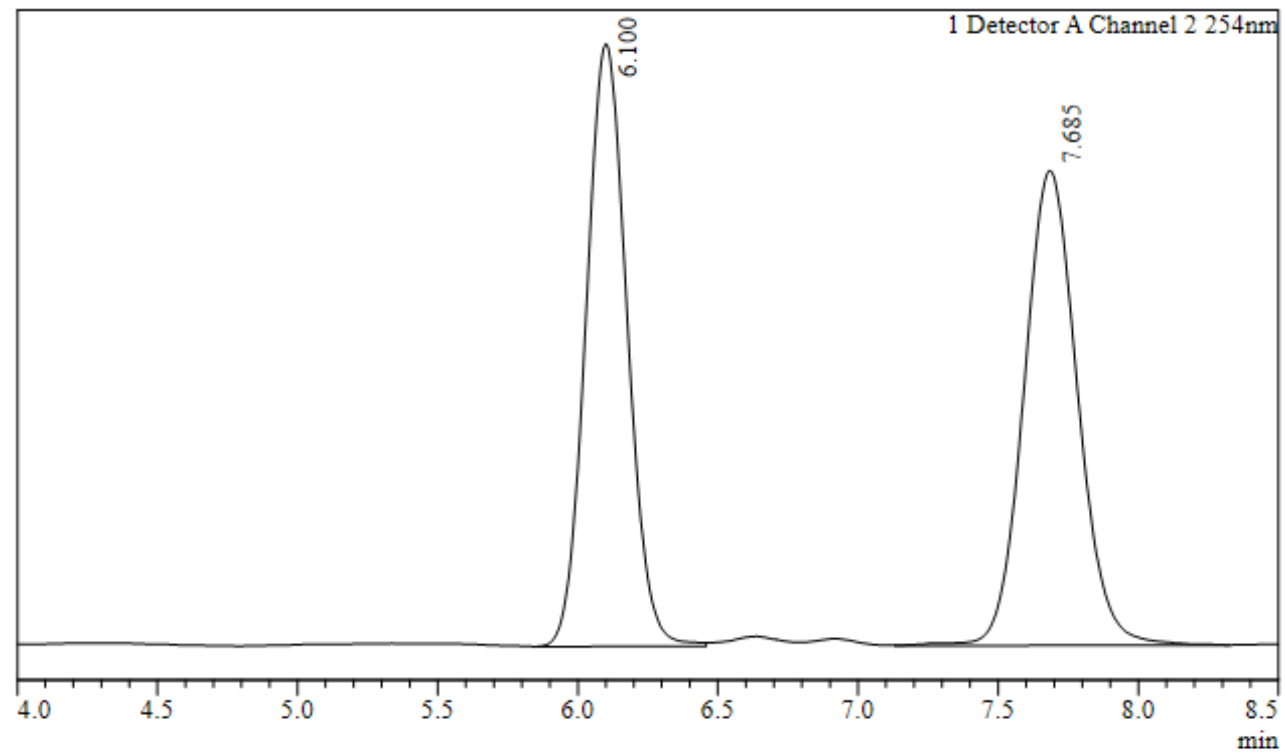

Peak Table

\begin{tabular}{|c|c|c|c|c|c|c|}
\hline \multicolumn{7}{|c|}{ Detector A Channel $2254 \mathrm{~nm}$} \\
\hline $\begin{array}{r}\text { Peak\# } \\
1\end{array}$ & $\begin{array}{r}\text { Ret. Time } \\
6.100\end{array}$ & $\begin{array}{l}\text { Area } \\
188112\end{array}$ & $\begin{array}{l}\text { Height } \\
18433\end{array}$ & $\begin{array}{l}\text { Area } \% \\
49.336\end{array}$ & $\begin{array}{l}\text { Conc. } \\
49.336\end{array}$ & Name \\
\hline 2 & 7.685 & 193176 & 14519 & 50.664 & 50.664 & \\
\hline Total & & 381288 & 32952 & 100.000 & & \\
\hline
\end{tabular}

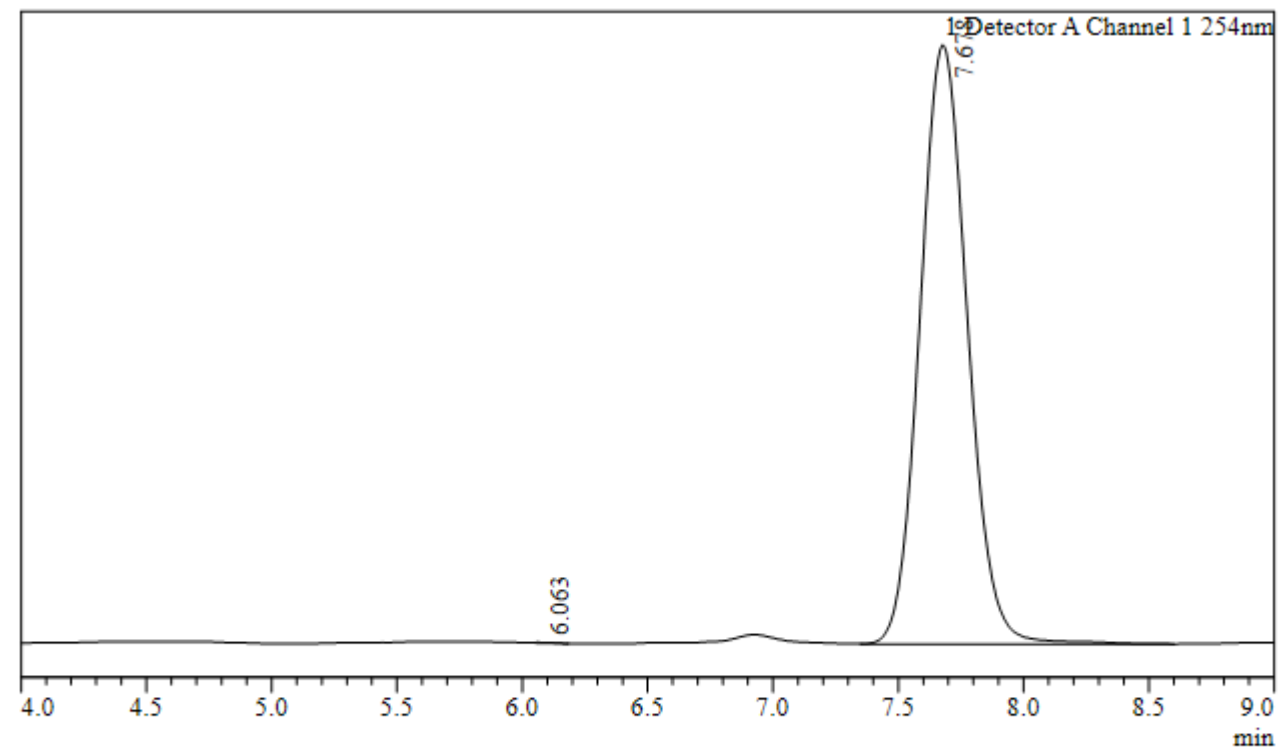

Peak Table

\begin{tabular}{|c|c|c|c|c|c|c|}
\hline Detector & Channel 1 & & & & & \\
\hline Peak\# & $\begin{array}{r}\text { Ret. Time } \\
6.063\end{array}$ & ${ }^{\text {Area }}{ }_{139}$ & ${ }^{\text {Height }_{25}}$ & $\begin{array}{l}\text { Area } \% \\
0.037\end{array}$ & $\begin{array}{l}\text { Conc. } \\
0.037\end{array}$ & Name \\
\hline 2 & 7.678 & 374415 & 28372 & 99.963 & 99.963 & \\
\hline Total & & 374554 & 28397 & 100.000 & & \\
\hline
\end{tabular}



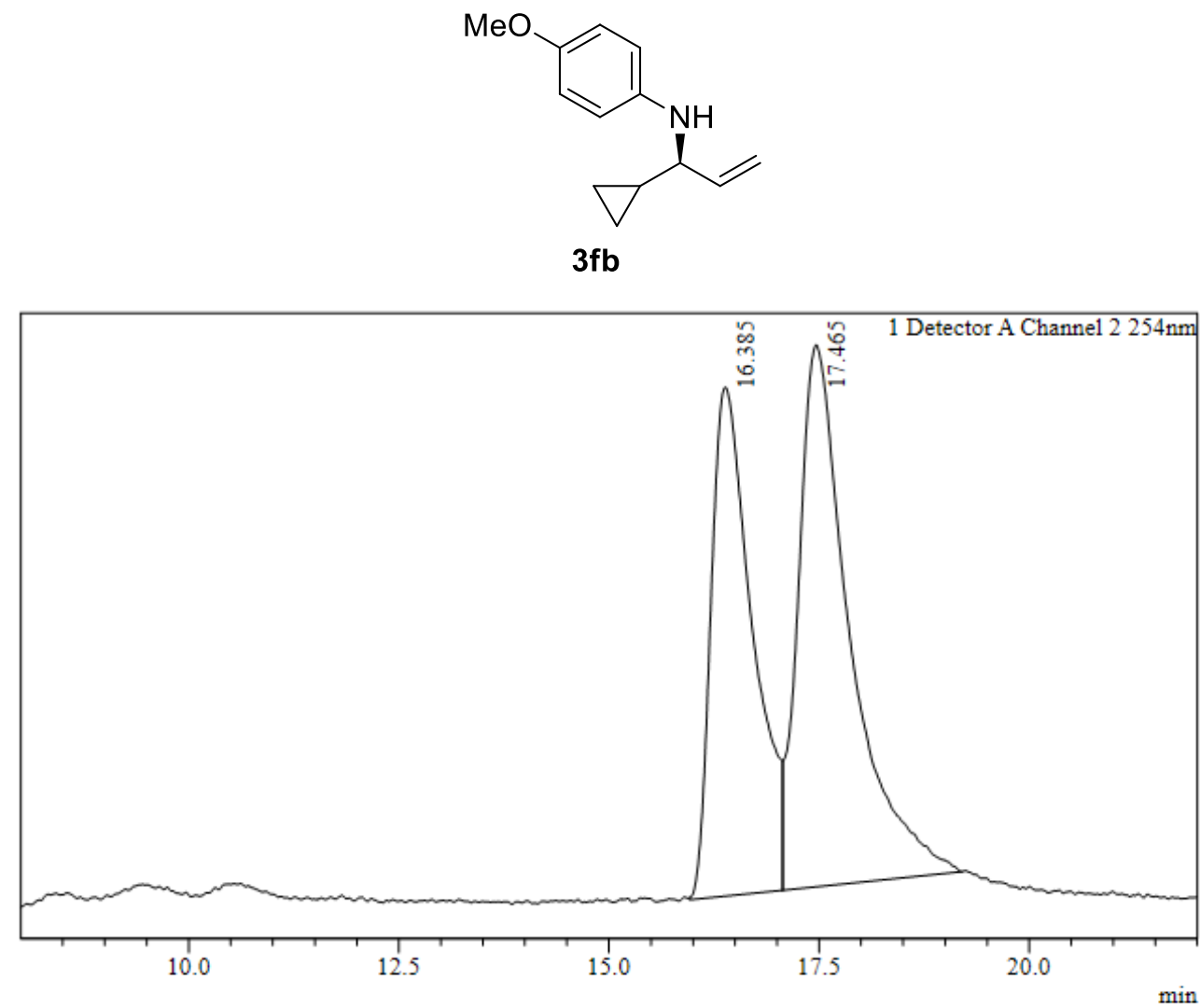

Peak Table
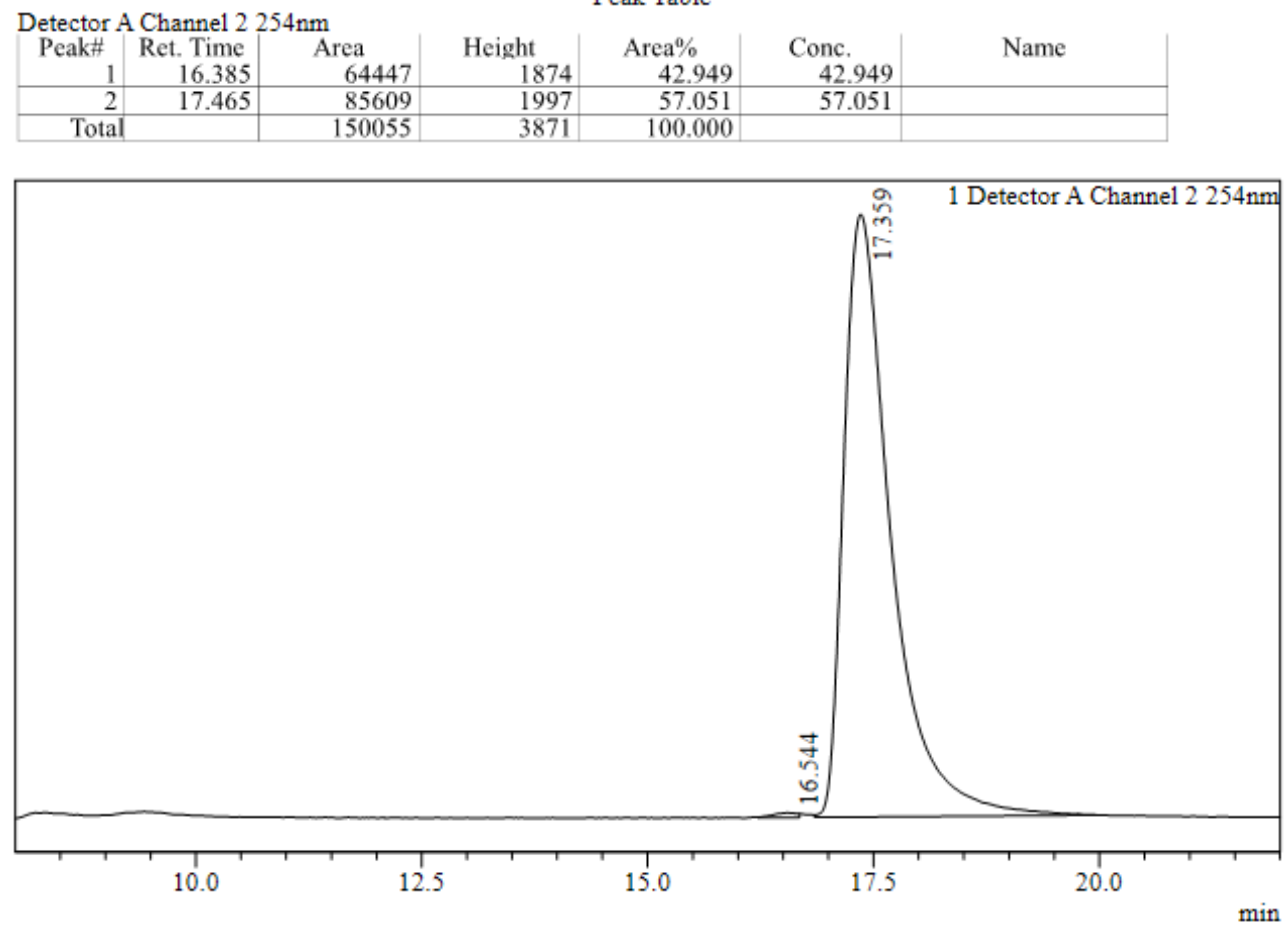

Peak Table

\begin{tabular}{|c|c|c|c|c|c|c|}
\hline \multicolumn{7}{|c|}{ Detector A Channel $2254 \mathrm{~nm}$} \\
\hline Peak\# & $\begin{array}{r}\text { Ret. Time } \\
16.544\end{array}$ & $\begin{array}{l}\text { Area } \\
\quad 2297\end{array}$ & $\begin{array}{l}\text { Height } \\
121\end{array}$ & $\begin{array}{c}\text { Area } \% \\
0.398\end{array}$ & $\begin{array}{l}\text { Conc. } \\
0.398\end{array}$ & Name \\
\hline 2 & 17.359 & 575509 & 15810 & 99.602 & 99.602 & \\
\hline Total & & 577806 & 15931 & 100.000 & & \\
\hline
\end{tabular}




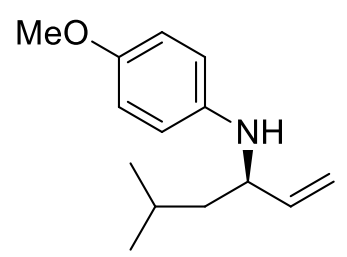

$3 g b$

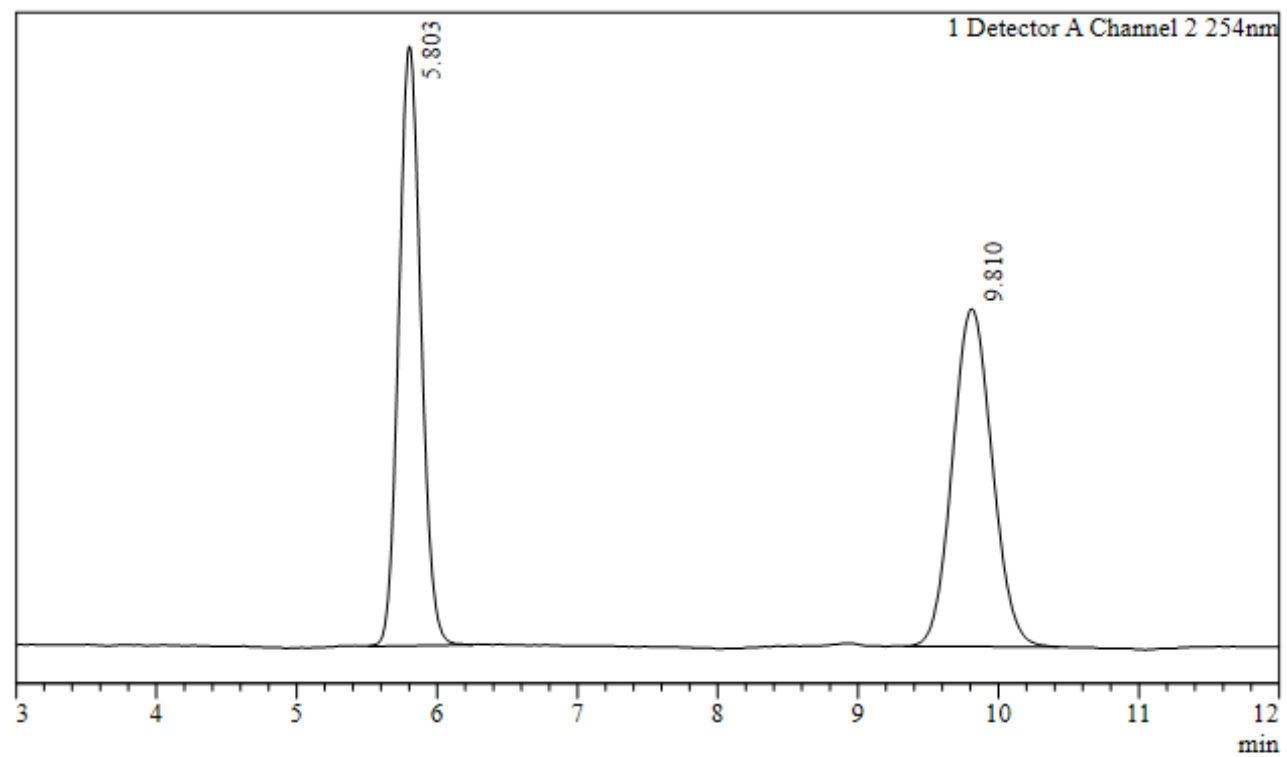

Peak Table

\begin{tabular}{|r|r|r|r|r|r|r|} 
Detector A Channel 2 & \multicolumn{2}{l}{. $54 \mathrm{~nm}$} & & & Name \\
\hline Peak\# & Ret. Time & Area & Height & Area\% & Conc. & Name \\
\hline 1 & 5.803 & 95791 & 8690 & 49.828 & 0.000 & \\
\hline 2 & 9.810 & 96453 & 4898 & 50.172 & 0.000 & \\
\hline Total & & 192244 & 13587 & 100.000 & & \\
\hline
\end{tabular}

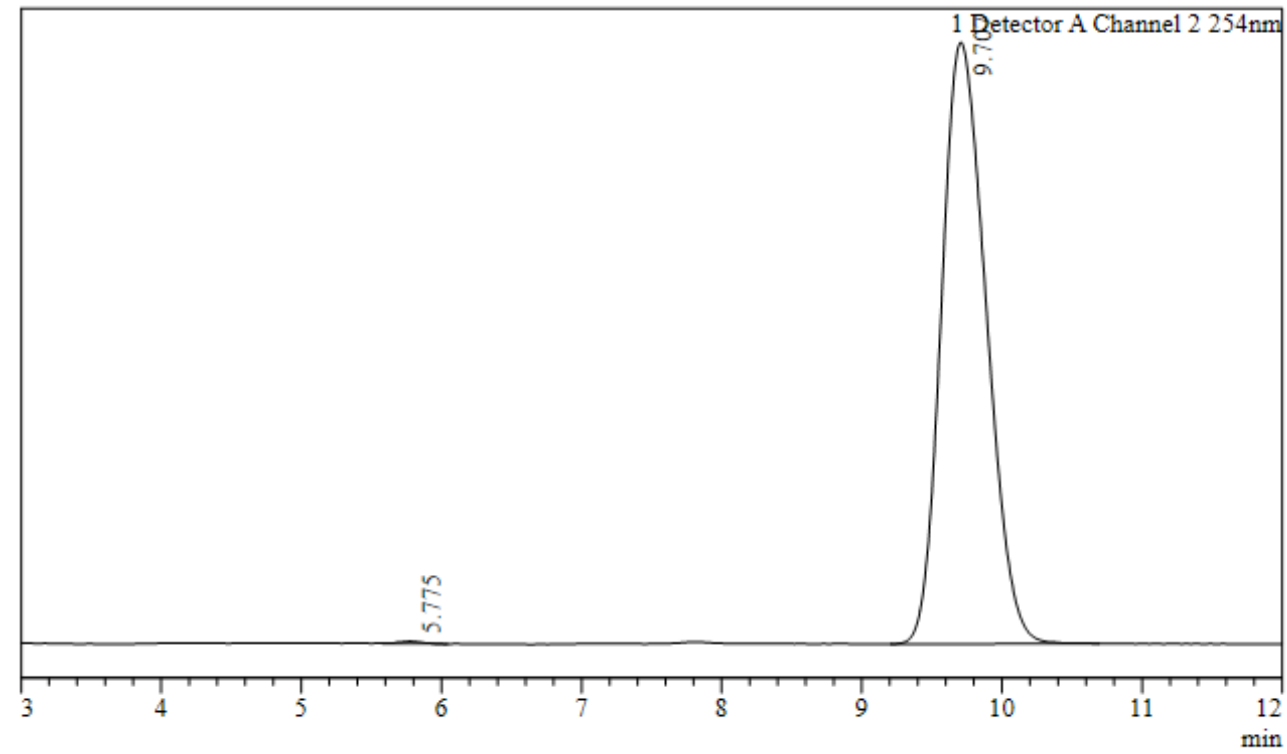

Peak Table

\begin{tabular}{|c|c|c|c|c|c|c|}
\hline Detector & Channel 2 & & & & & \\
\hline Peak\# & $\begin{array}{l}\text { Ret. Time } \\
5.775\end{array}$ & ${ }_{1207}$ & $\begin{array}{l}\text { Height } \\
116\end{array}$ & $\begin{array}{l}\text { Area } \% \\
0.192\end{array}$ & $\begin{array}{l}\text { Conc. } \\
0.192\end{array}$ & Name \\
\hline 2 & 9.707 & 627112 & 28218 & 99.808 & 99.808 & \\
\hline Total & & 628319 & 28334 & 100.000 & & \\
\hline
\end{tabular}



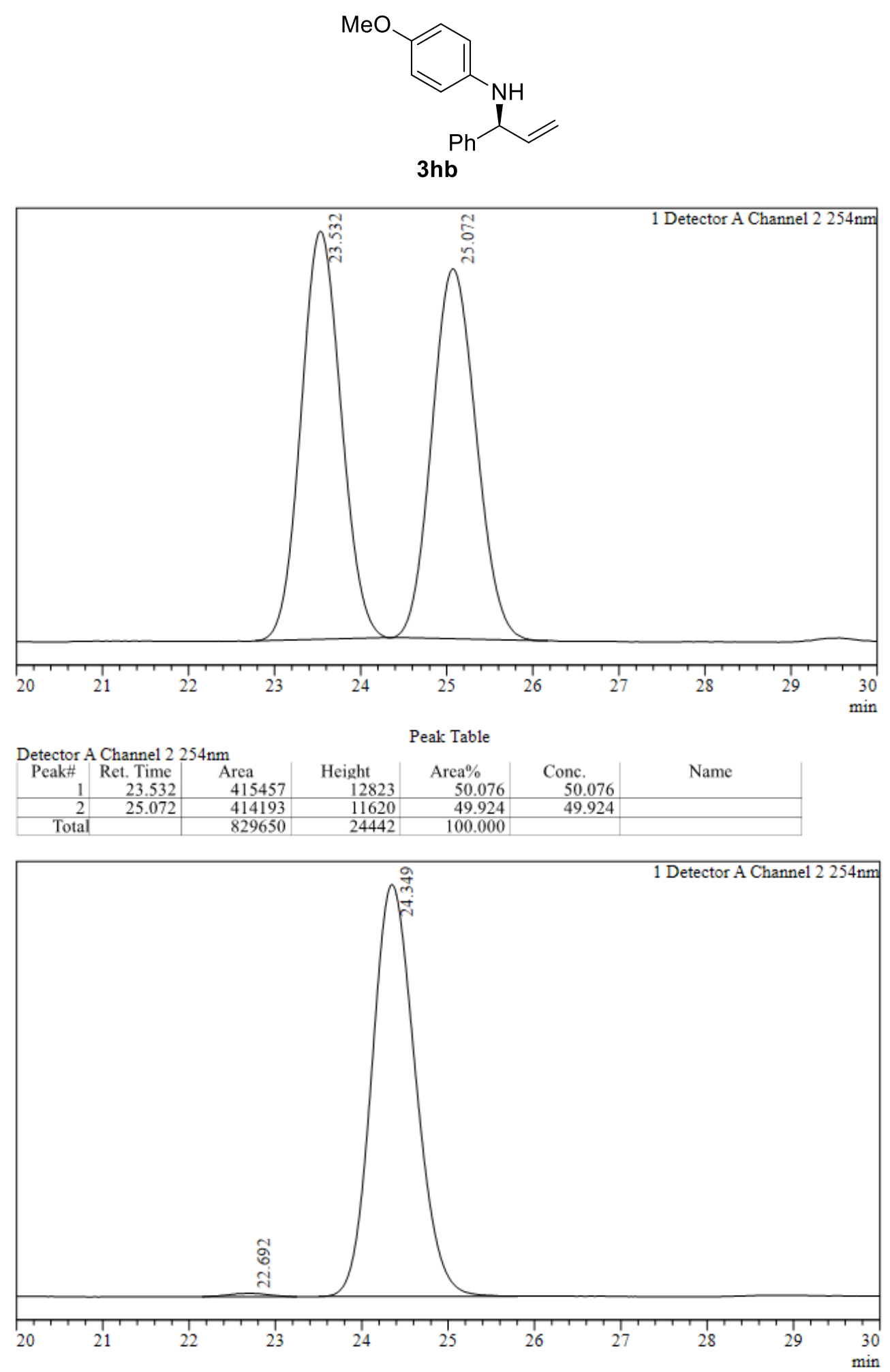

Peak Table

\begin{tabular}{|c|c|c|c|c|c|c|}
\hline \multicolumn{7}{|c|}{ Detector A Channel $2254 \mathrm{~nm}$} \\
\hline Peak\# & $\begin{array}{r}\text { Ret. Time } \\
22.692\end{array}$ & ${ }^{\text {Area }}{ }_{6617}$ & $\begin{array}{l}\text { Height } \\
216\end{array}$ & $\begin{array}{c}\text { Area } \% \\
0.720\end{array}$ & $\begin{array}{l}\text { Conc. } \\
0.720\end{array}$ & Name \\
\hline 2 & 24.349 & 912154 & 26079 & 99.280 & 99.280 & \\
\hline Total & & 918771 & 26295 & 100.000 & & \\
\hline
\end{tabular}



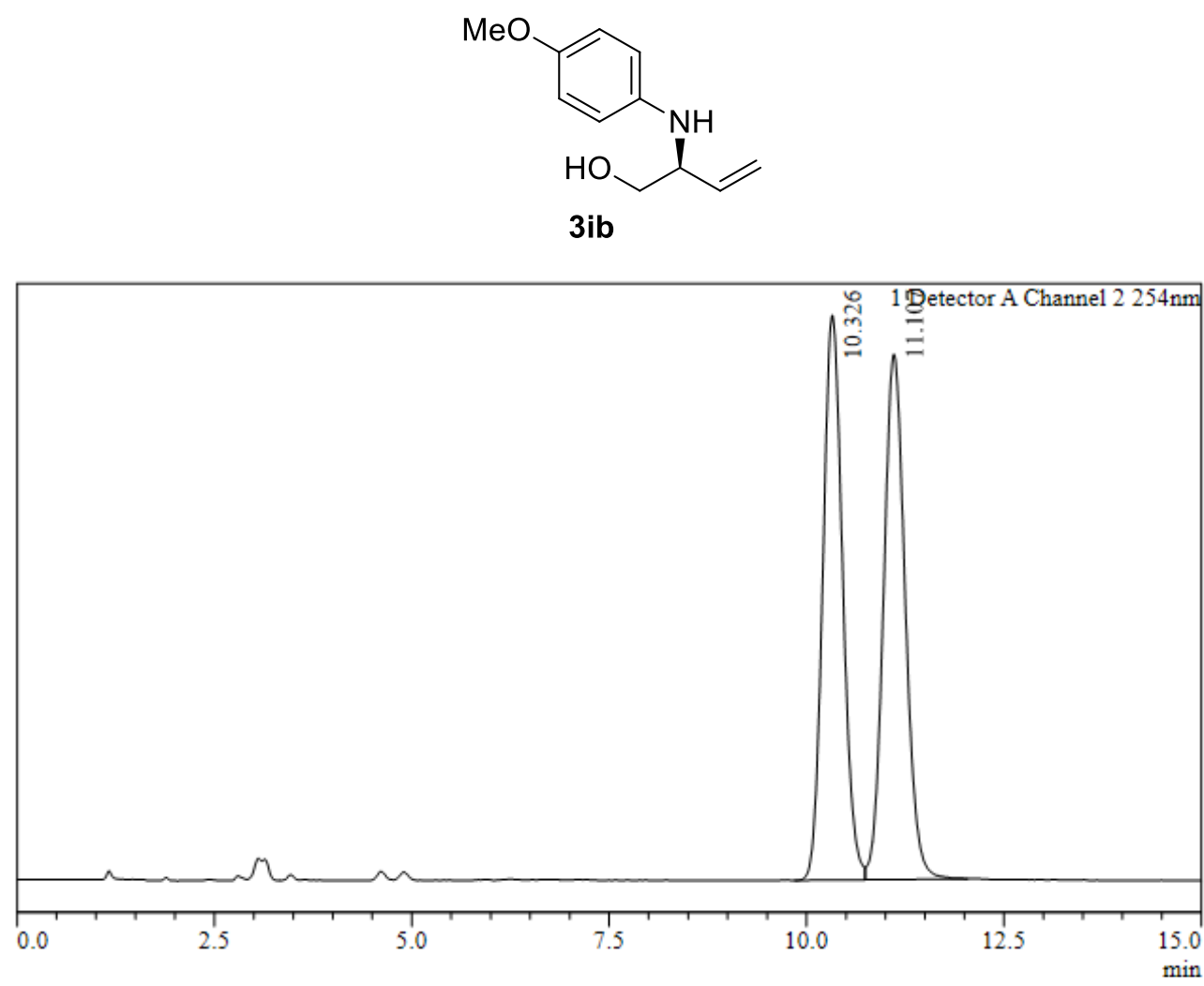

Peak Table

\begin{tabular}{|c|c|c|c|c|c|c|}
\hline Detector & Channel 2 & & & & & \\
\hline Peak\# & Ret. Time & Area & $\begin{array}{l}\text { Height } \\
18453\end{array}$ & $\begin{array}{l}\text { Area } \% \\
49616\end{array}$ & $\begin{array}{l}\text { Conc. } \\
49616\end{array}$ & Name \\
\hline 2 & 11.107 & 327216 & 17159 & 50.384 & 50.384 & \\
\hline Total & & 649448 & 35612 & 100.000 & & \\
\hline
\end{tabular}

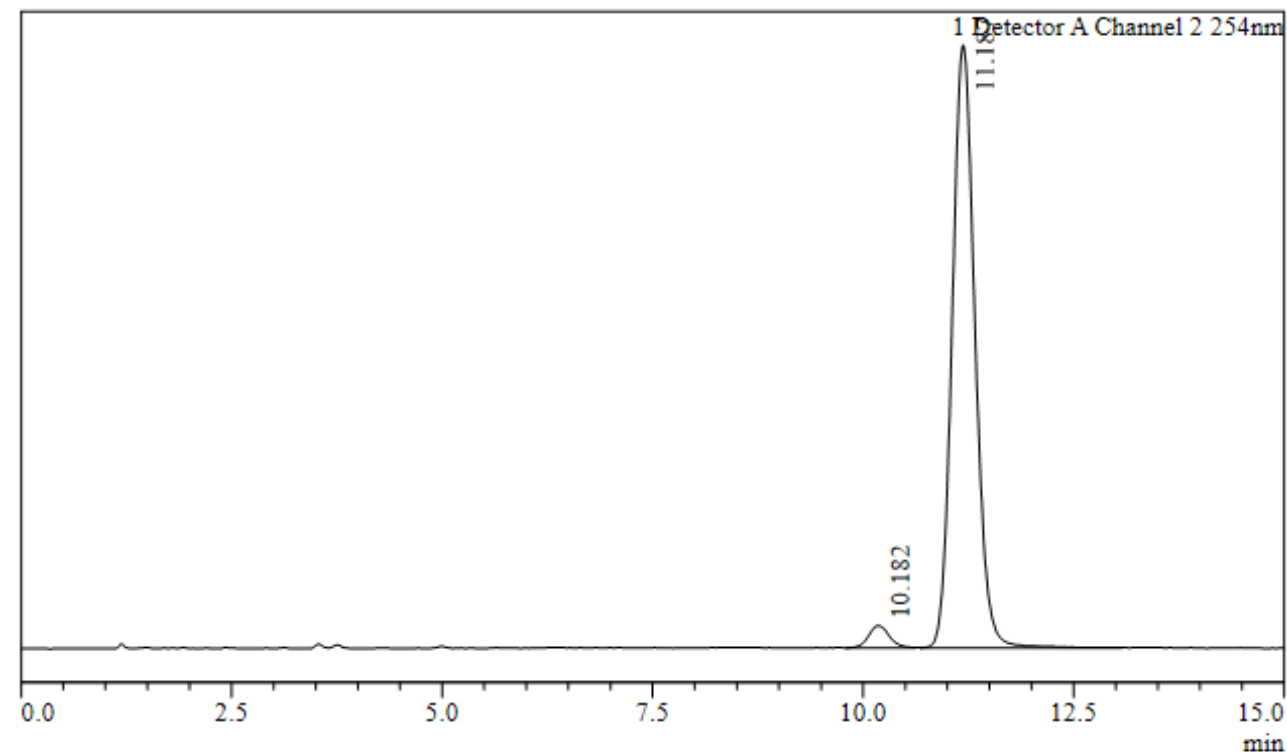

Peak Table

\begin{tabular}{|c|c|c|c|c|c|c|}
\hline \multicolumn{7}{|c|}{ Detector A Channel $2254 \mathrm{~nm}$} \\
\hline Peak\# & $\begin{array}{r}\text { Ret. Time } \\
10.182\end{array}$ & $\begin{array}{l}\text { Area } \\
\quad 12990\end{array}$ & $\begin{array}{l}\text { Height } \\
\quad 759\end{array}$ & $\begin{array}{l}\text { Area } \% \\
3.252\end{array}$ & $\begin{array}{l}\text { Conc. } \\
3.252\end{array}$ & Name \\
\hline 2 & 11.187 & 386433 & 20536 & 96.748 & 96.748 & \\
\hline Total & & 399423 & 21294 & 100.000 & & \\
\hline
\end{tabular}




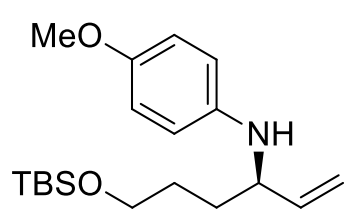

3jb

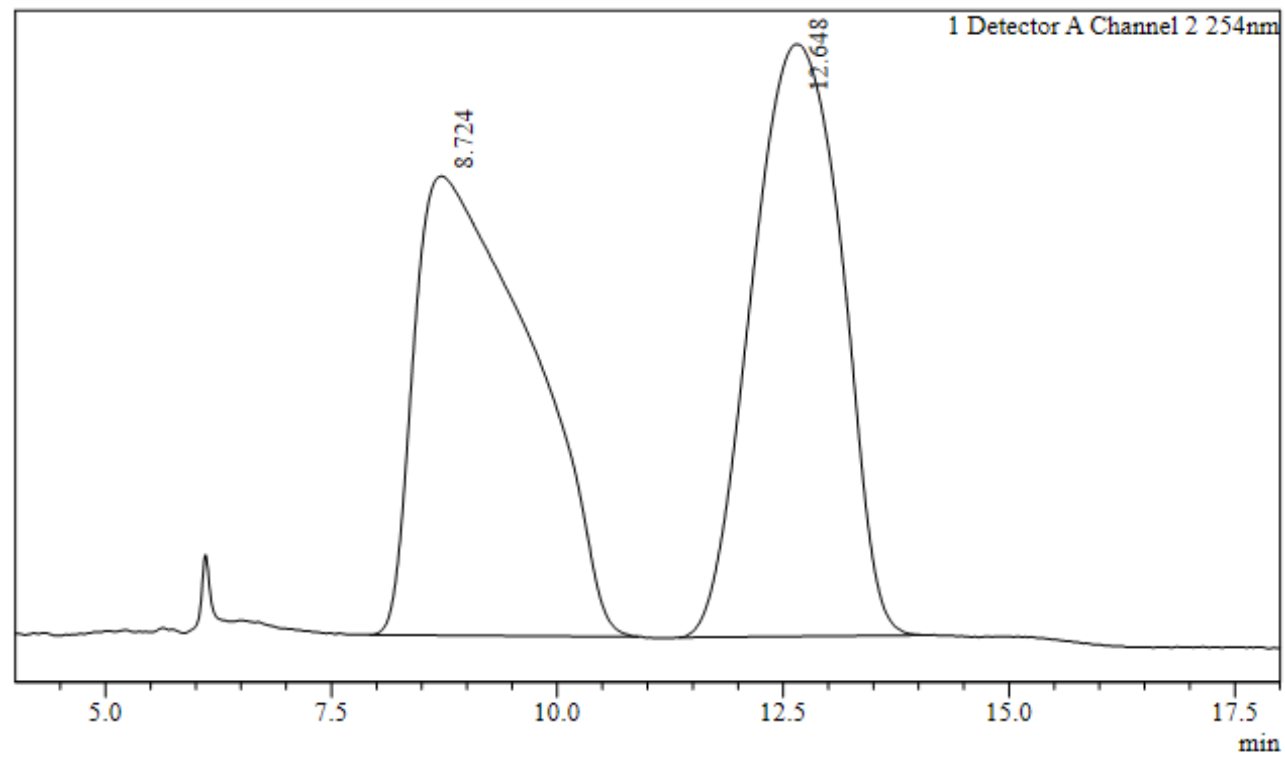

Peak Table
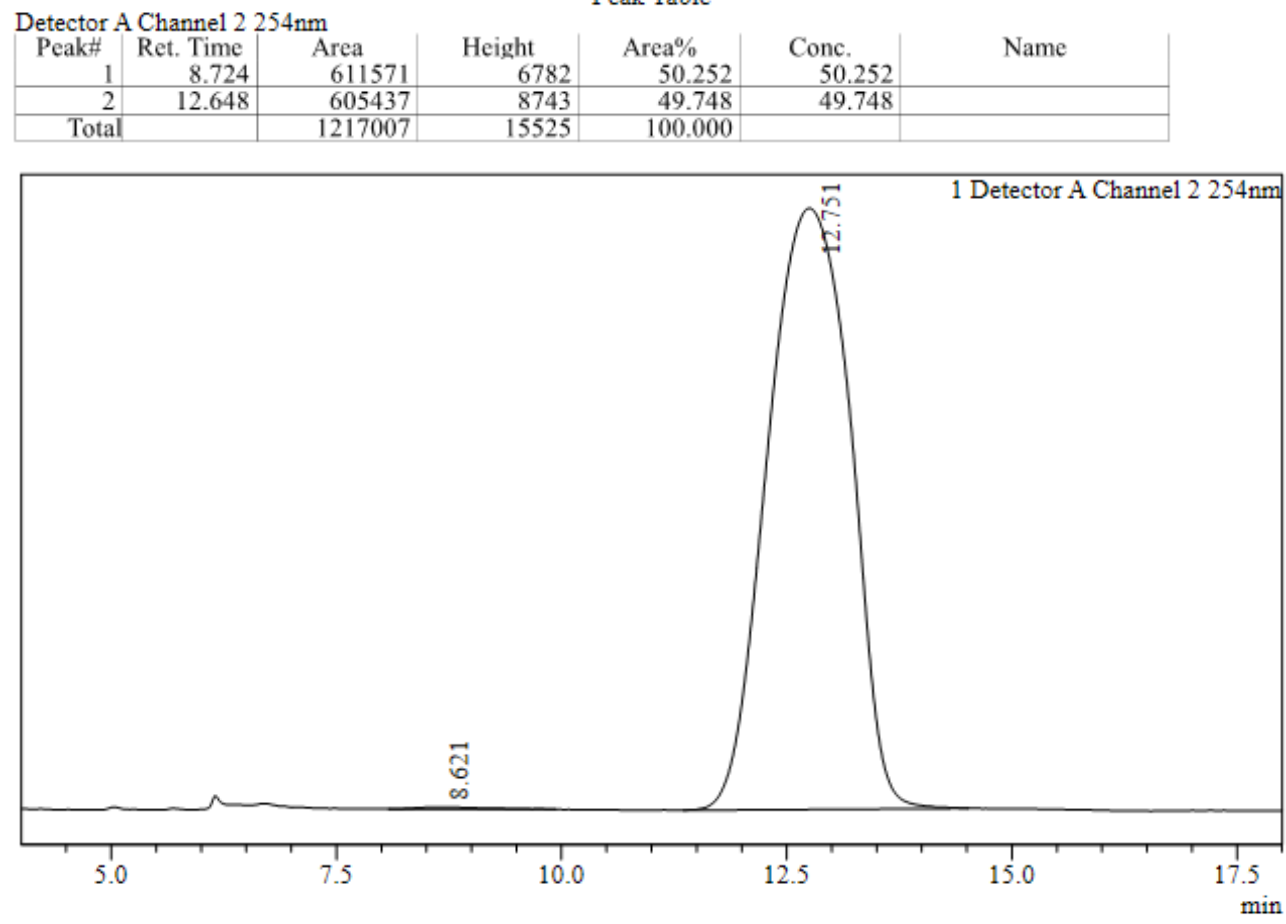

Peak Table

\begin{tabular}{|c|c|c|c|c|c|c|}
\hline \multicolumn{7}{|c|}{ Detector A Channel $2254 \mathrm{~nm}$} \\
\hline Peak\# $_{1}$ & $\begin{array}{r}\text { Ret. Time } \\
8.621\end{array}$ & $\begin{array}{l}\text { Area } \\
5611\end{array}$ & $\begin{array}{l}\text { Height } \\
\end{array} 98$ & $\begin{array}{l}\text { Area } \% \\
0.299\end{array}$ & $\begin{array}{l}\text { Conc. } \\
0.299\end{array}$ & Name \\
\hline 2 & 12.751 & 1872297 & 29302 & 99.701 & 99.701 & \\
\hline Total & & 1877908 & 29400 & 100.000 & & \\
\hline
\end{tabular}




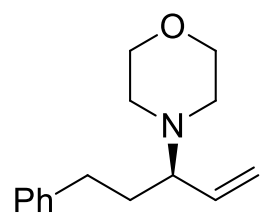

$7 \mathrm{ka}$

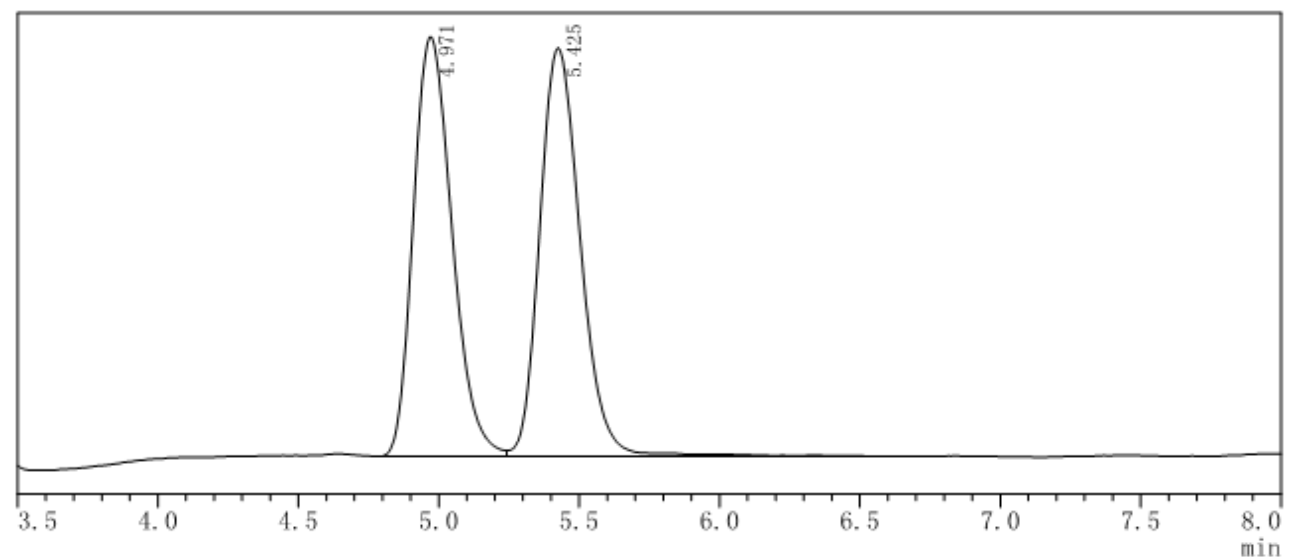

\begin{tabular}{|c|c|c|c|c|c|}
\hline \multicolumn{6}{|l|}{ set } \\
\hline Peak NO. & Retention Time & $\underset{15254}{\text { Height }}$ & $\begin{array}{r}\text { Height } \% \\
50.639\end{array}$ & $\underset{146756}{\text { Area }}$ & $\begin{array}{l}\text { Area } \% \\
\quad 49.481\end{array}$ \\
\hline & 5.425 & 14869 & 49.361 & 149837 & 50.519 \\
\hline 总方 & & 30123 & 100.000 & 296593 & 100.000 \\
\hline
\end{tabular}

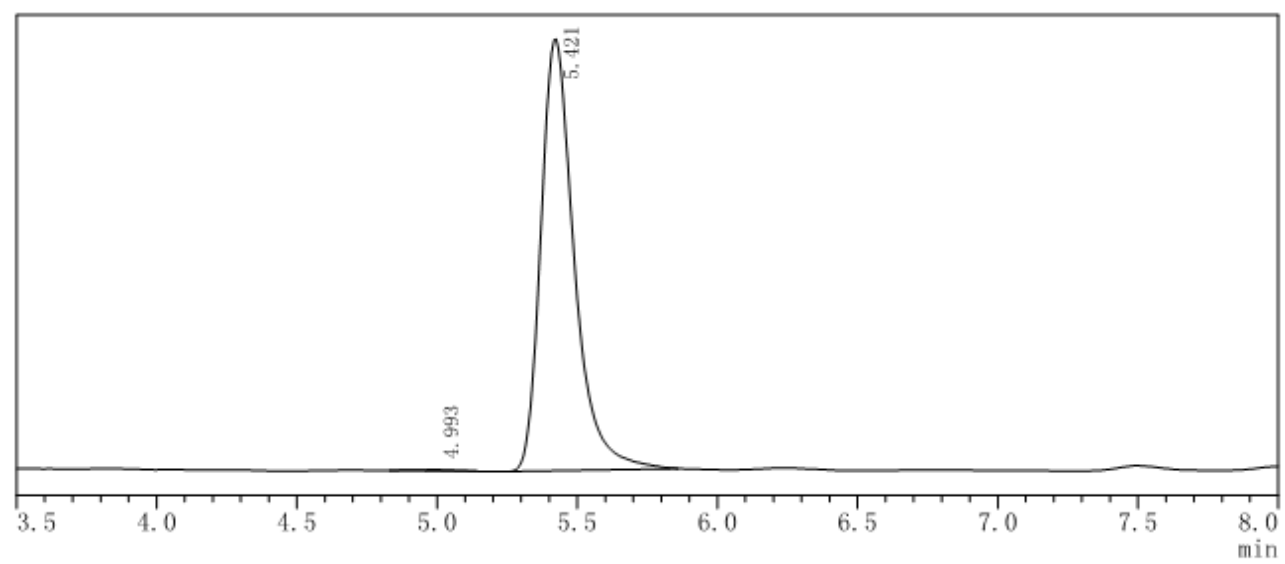

\begin{tabular}{r|r|r|r|r|r|} 
检测器A Ch1 254nm & & & \\
Peak NO. & Retention Time & Height & Height\% & Area & Area\% \\
\hline 1 & 4.993 & 125 & 0.302 & 1022 & 0.297 \\
\hline 2 & 5.421 & 41198 & 99.698 & 342345 & 99.703 \\
\hline 总妍 & & 41323 & 100.000 & 343366 & 100.000 \\
\hline
\end{tabular}



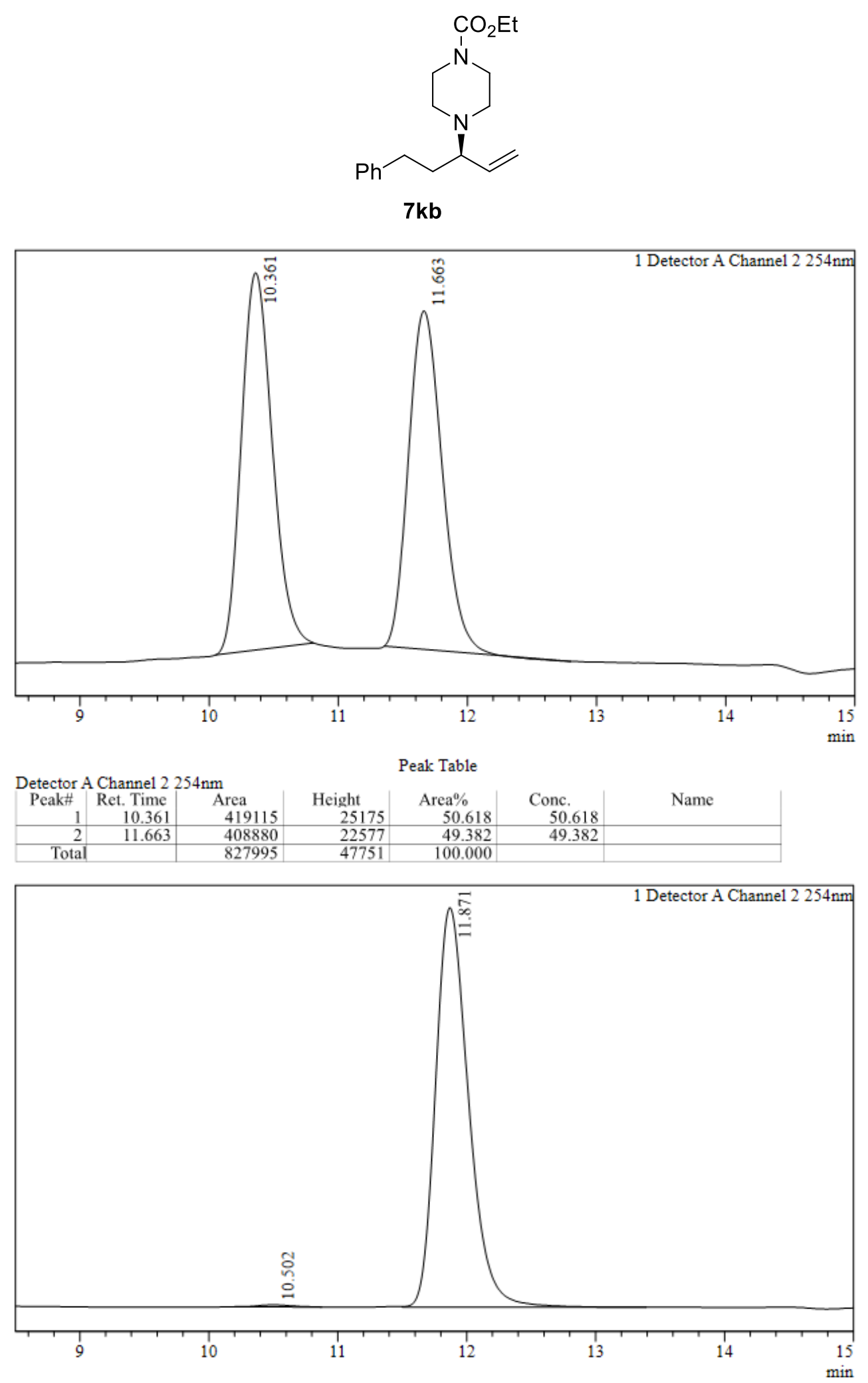

Peak Table

\begin{tabular}{|r|r|r|r|r|r|r|}
\multicolumn{2}{c}{ Detector A Channe1 2 $254 \mathrm{~nm}$} & \multicolumn{3}{c|}{ Peak Table } \\
Peak\# & Ret. Time & Area & Height & Area\% & Conc. & Name \\
\hline 1 & 10.502 & 5447 & 310 & 0.496 & 0.496 & \\
\hline 2 & 11.871 & 1092791 & 61393 & 99.504 & 99.504 & \\
\hline Total & & 1098238 & 61703 & 100.000 & & \\
\hline
\end{tabular}




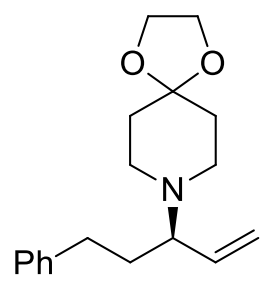

$7 \mathrm{kc}$

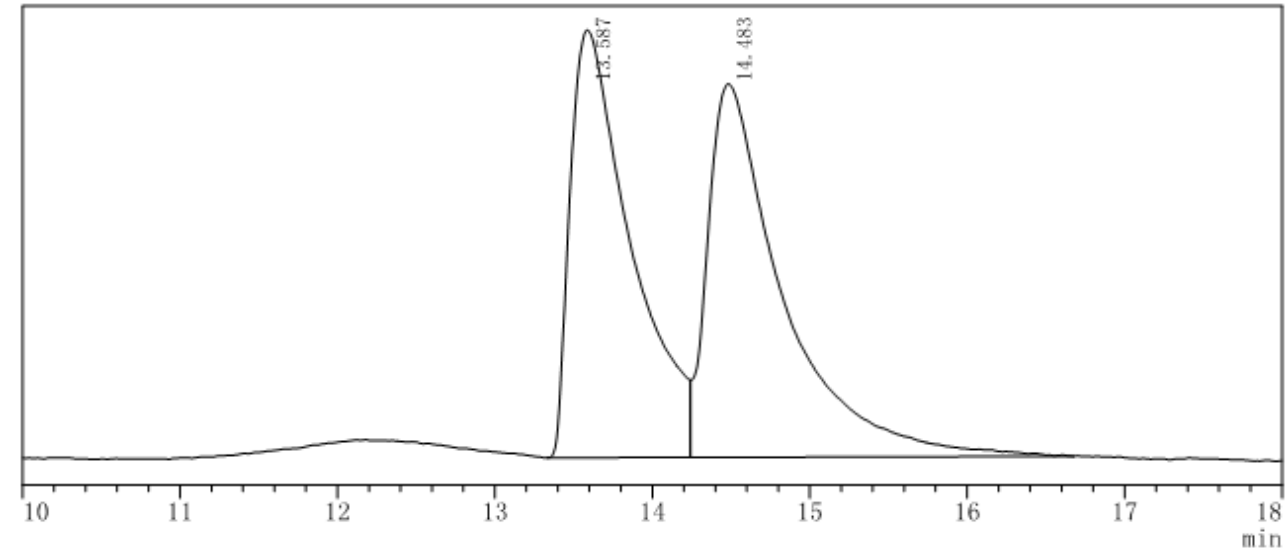

\begin{tabular}{|c|c|c|c|c|c|}
\hline${ }_{1}$ & $\begin{array}{r}\text { Retention Time } \\
13.587\end{array}$ & $\begin{array}{r}\text { Height } \\
8579\end{array}$ & $\begin{array}{r}\text { Height } \% \\
53.385\end{array}$ & $\begin{array}{l}\text { Area } \\
230630\end{array}$ & $\begin{array}{l}\text { Area } \% \\
48.136\end{array}$ \\
\hline 2 & 14.483 & 7491 & 46.615 & 248493 & 51.864 \\
\hline 甞计 & & 16070 & 100.000 & 479123 & 100.000 \\
\hline
\end{tabular}

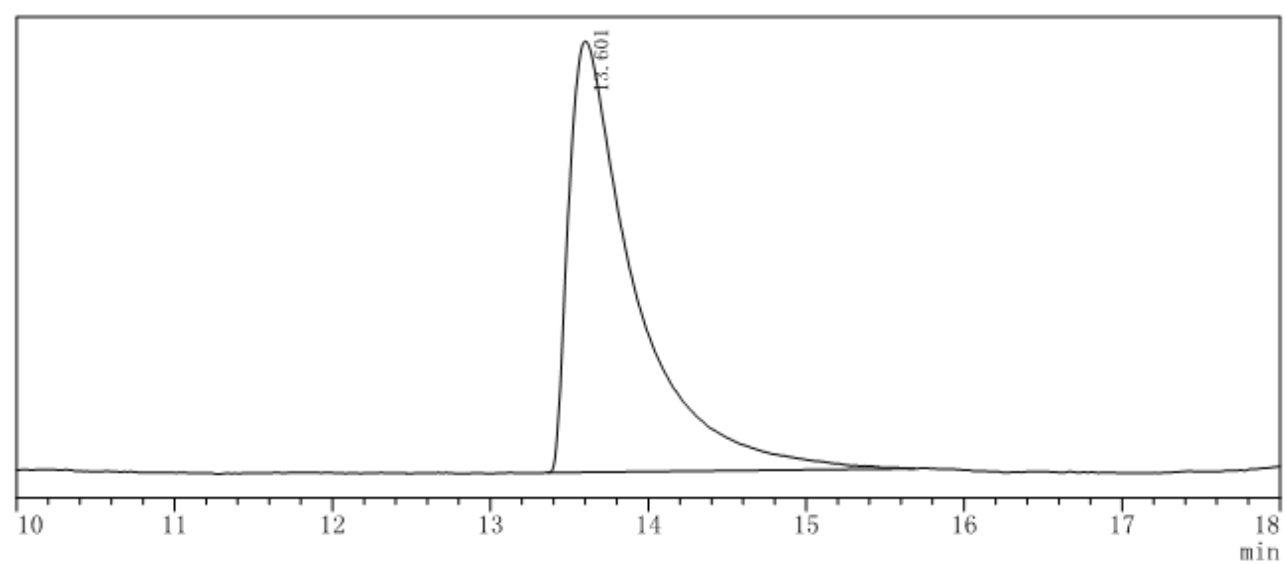

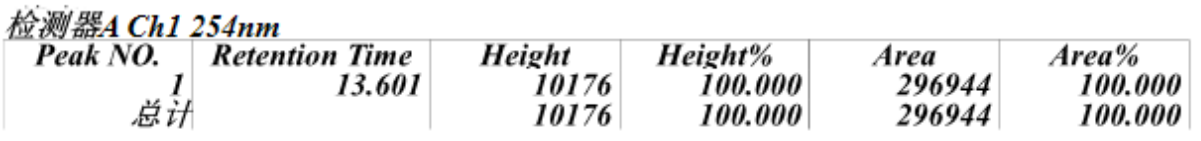




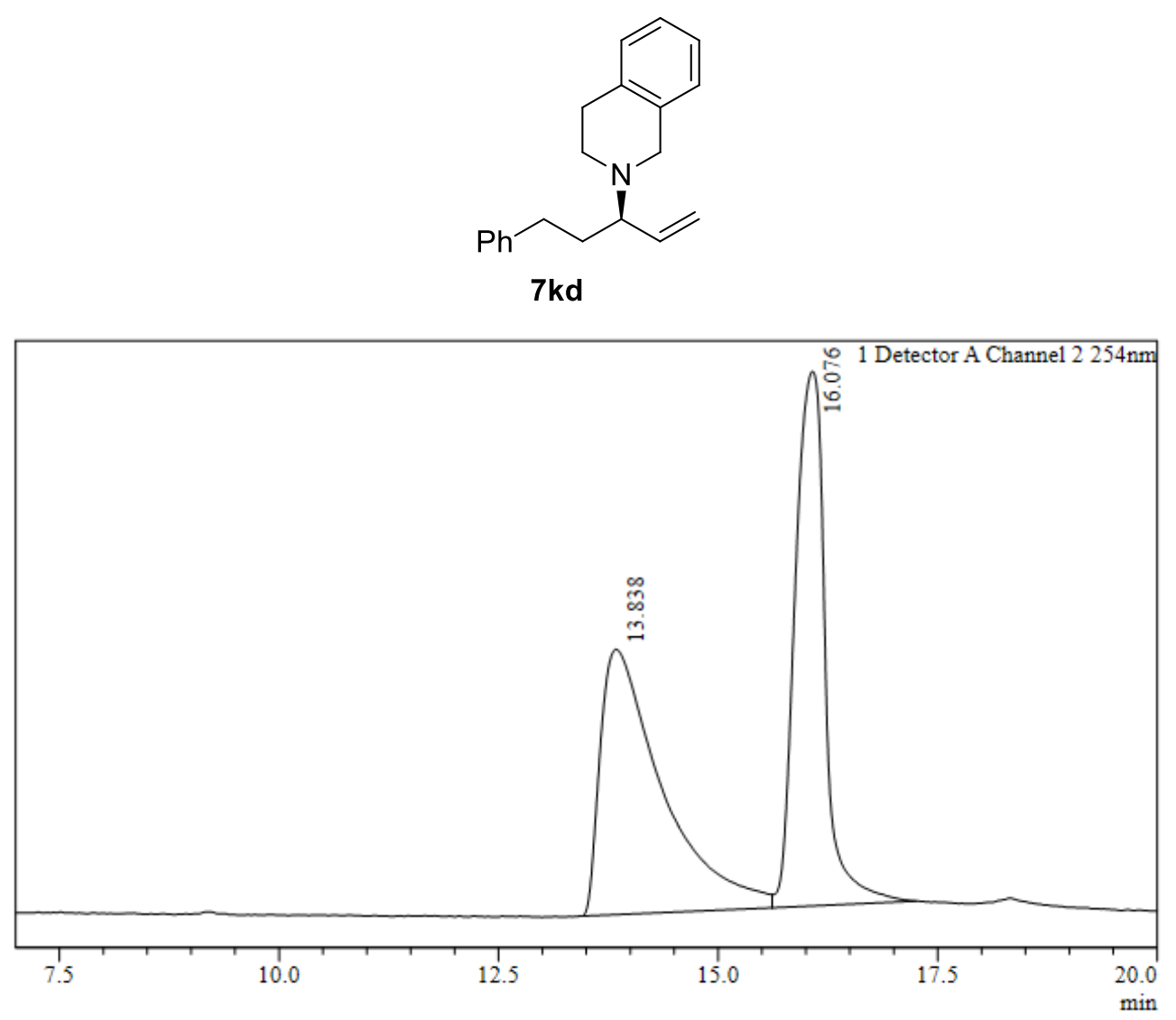

Peak Table
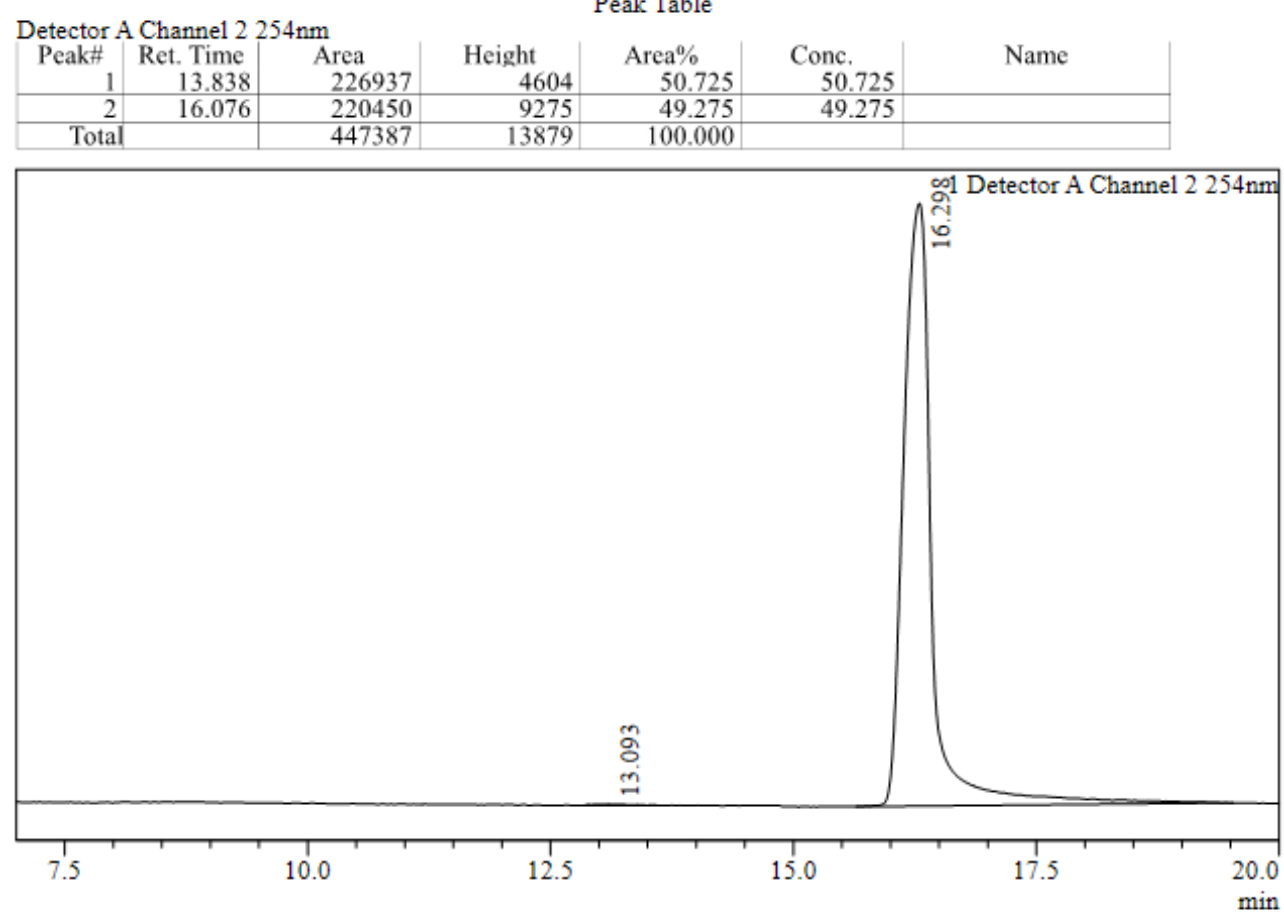

Peak Table

\begin{tabular}{|c|c|c|c|c|c|c|}
\hline \multirow{2}{*}{\multicolumn{7}{|c|}{ Detector A Channel $2254 \mathrm{~nm}$}} \\
\hline & & & & & & \\
\hline $\begin{array}{c}\text { Peak\# } \\
1\end{array}$ & $\begin{array}{r}\text { Ret. Time } \\
13.093\end{array}$ & $\begin{array}{l}\text { Area } \\
\\
\end{array}$ & $\begin{array}{l}\text { Height } \\
\end{array}$ & $\begin{array}{l}\text { Area } \% \\
0.256\end{array}$ & $\begin{array}{l}\text { Conc. } \\
0.256\end{array}$ & Name \\
\hline 2 & 16.298 & 287630 & 14197 & 99.744 & 99.744 & \\
\hline Total & & 288369 & 14227 & 100.000 & & \\
\hline
\end{tabular}



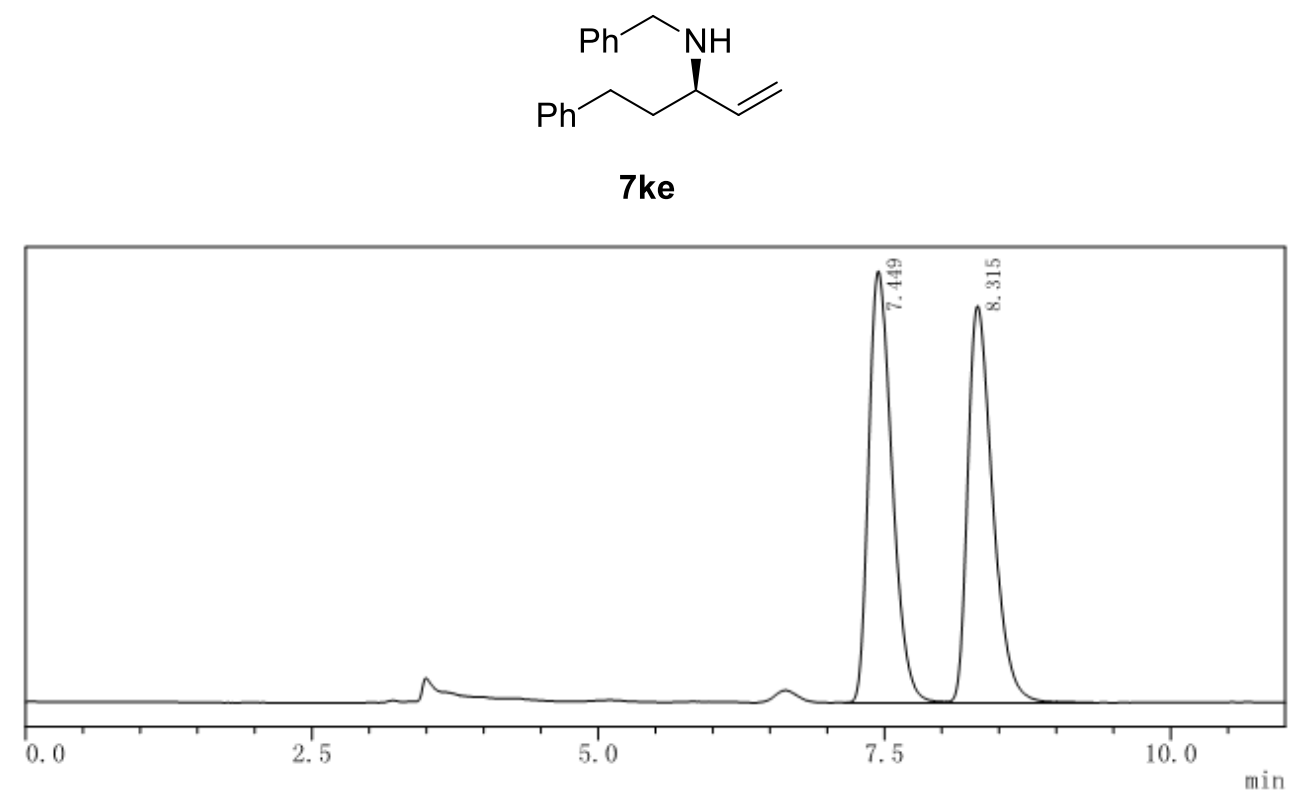

\begin{tabular}{|c|c|c|c|c|c|}
\hline Peak NO. & Retention Time & $\underset{27775}{\text { Height }}$ & $\begin{array}{l}\text { Height } \% \\
52.104\end{array}$ & $\begin{array}{l}\text { Area } \\
394708\end{array}$ & $\begin{array}{l}\text { Area } \% \\
50.418\end{array}$ \\
\hline 2 & 8.315 & 25532 & 47.896 & 388157 & 49.582 \\
\hline 总计 & & 53307 & 100.000 & 782865 & 100.000 \\
\hline
\end{tabular}

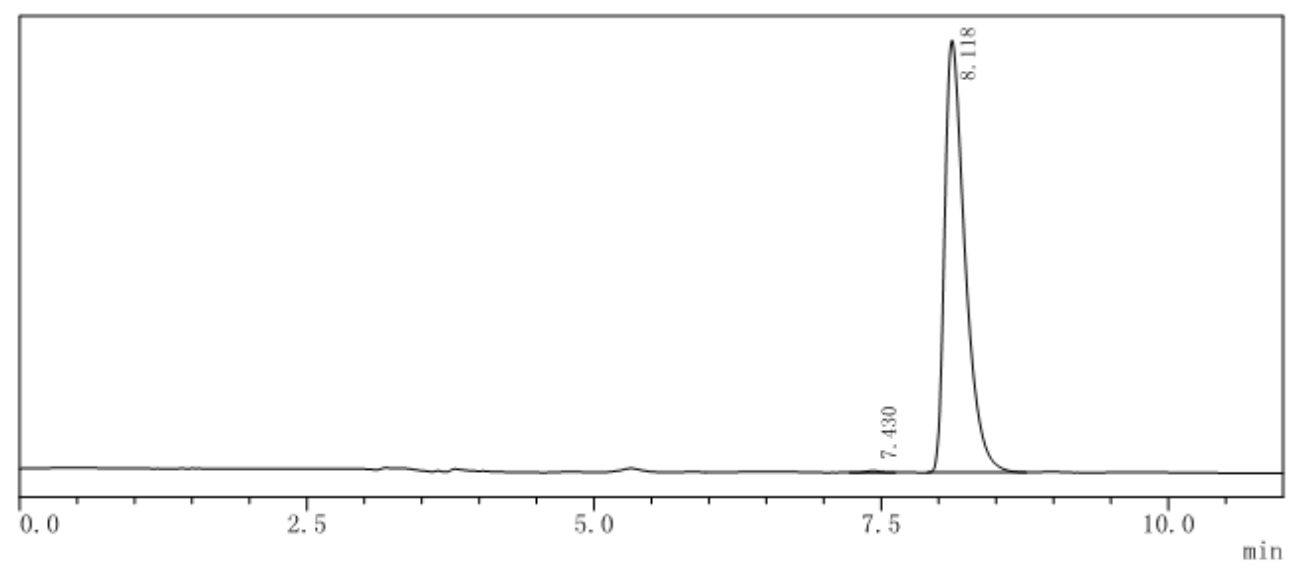

\begin{tabular}{|r|r|r|r|r|r|}
\multicolumn{2}{|c|}{ 检测器A Ch1 254nm } & & & \\
Peak NO. & Retention Time & Height & Height\% & Area & Area\% \\
\hline 1 & 7.430 & 184 & 0.497 & 2160 & 0.472 \\
\hline 2 & 8.118 & 36741 & 99.503 & 455615 & 99.528 \\
\hline 总计 & & 36925 & 100.000 & 457775 & 100.000 \\
\hline
\end{tabular}



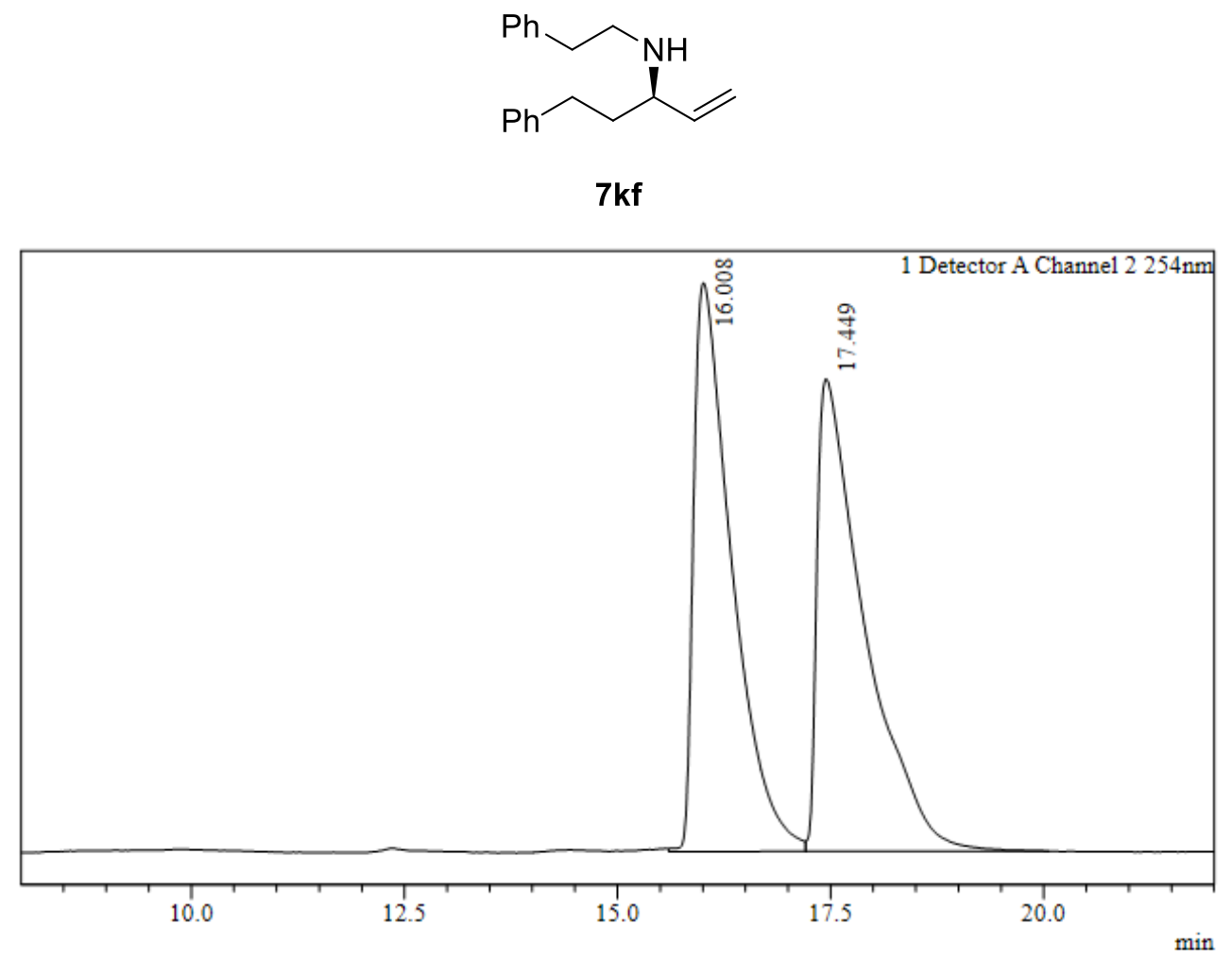

Peak Table
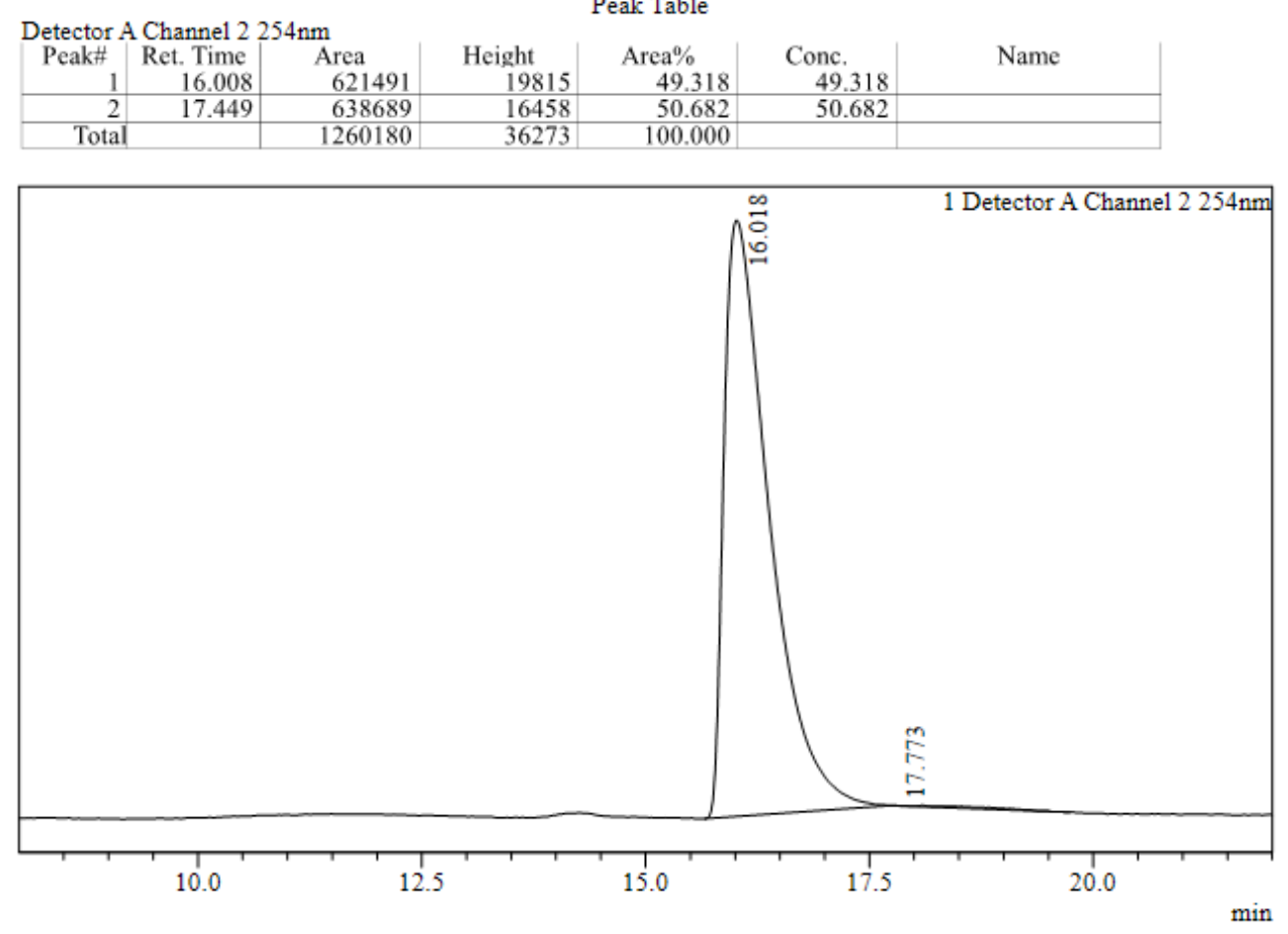

Peak Table

\begin{tabular}{|c|c|c|c|c|c|c|}
\hline \multirow{2}{*}{\multicolumn{7}{|c|}{ Detector A Channel $2254 \mathrm{~nm}$}} \\
\hline & & & & & & \\
\hline $\begin{array}{c}\text { Peak\# } \\
1\end{array}$ & $\begin{array}{r}\text { Ret. Time } \\
16.018\end{array}$ & $\begin{array}{l}\text { Area } \\
414801\end{array}$ & $\begin{array}{l}\text { Height } \\
11724\end{array}$ & $\begin{array}{l}\text { Area } \% \\
\quad 99.510\end{array}$ & $\begin{array}{l}\text { Conc. } \\
99.510\end{array}$ & Name \\
\hline 2 & 17.773 & 2044 & 7 & 0.490 & 0.490 & \\
\hline Total & & 416845 & 11731 & 100.000 & & \\
\hline
\end{tabular}




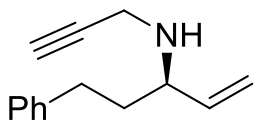

$7 \mathrm{~kg}$

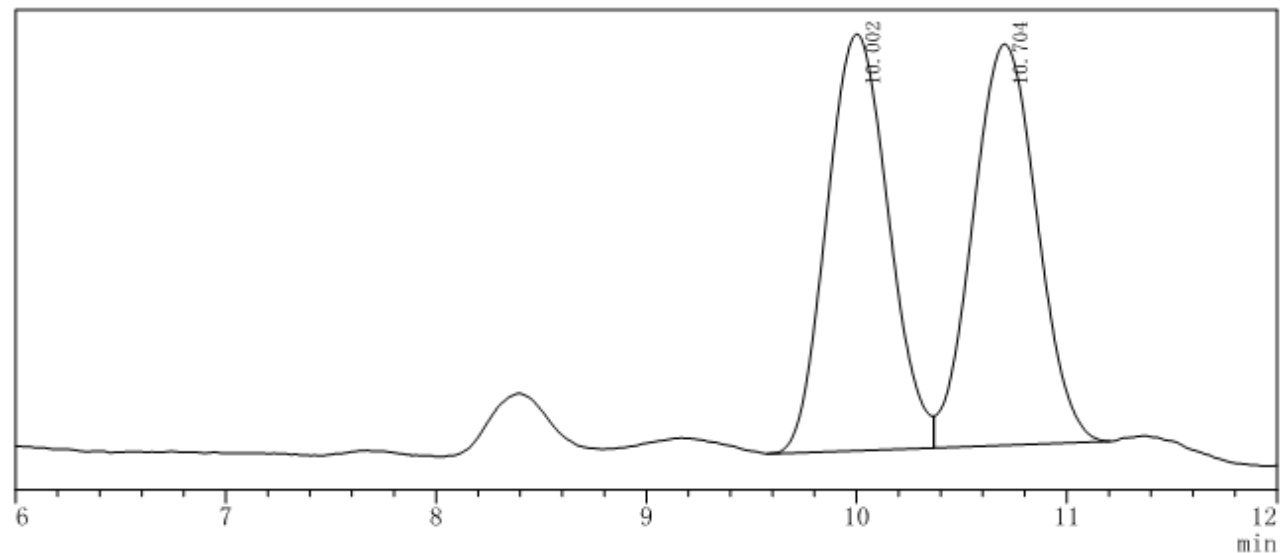

\begin{tabular}{|c|c|c|c|c|c|}
\hline Peak NO. & Retention Time & $\begin{array}{l}\text { Height } \\
5650\end{array}$ & $\begin{array}{c}\text { Height } \% \\
50.944\end{array}$ & $\begin{array}{l}\text { Area } \\
\quad 115418\end{array}$ & $\begin{array}{l}\text { Area } \% \\
49.933\end{array}$ \\
\hline 2 & 10.704 & 5441 & 49.056 & 115729 & 50.067 \\
\hline 急计 & & 11091 & 100.000 & 231147 & 100.000 \\
\hline
\end{tabular}

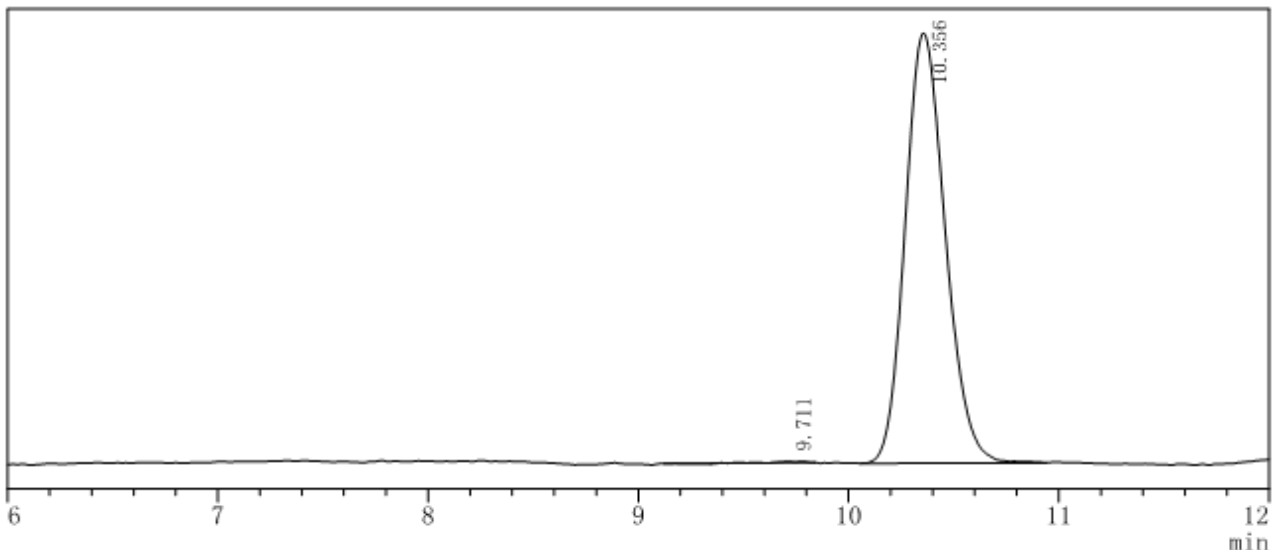

\begin{tabular}{|c|c|c|c|c|c|}
\hline${ }_{1}$ & $\begin{array}{r}\text { Retention Time } \\
9.711\end{array}$ & $\begin{array}{l}\text { Height } \\
\\
\end{array}$ & $\begin{array}{r}\text { Height } \% \\
0.314\end{array}$ & ${ }_{203}$ & $\begin{array}{l}\text { Area } \% \\
\quad 0.191\end{array}$ \\
\hline 2 & 10.356 & 7981 & 99.686 & 106022 & 99.809 \\
\hline 总计 & & 8006 & 100.000 & 106225 & 100.000 \\
\hline
\end{tabular}




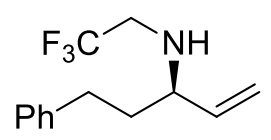

$7 \mathbf{k j}$
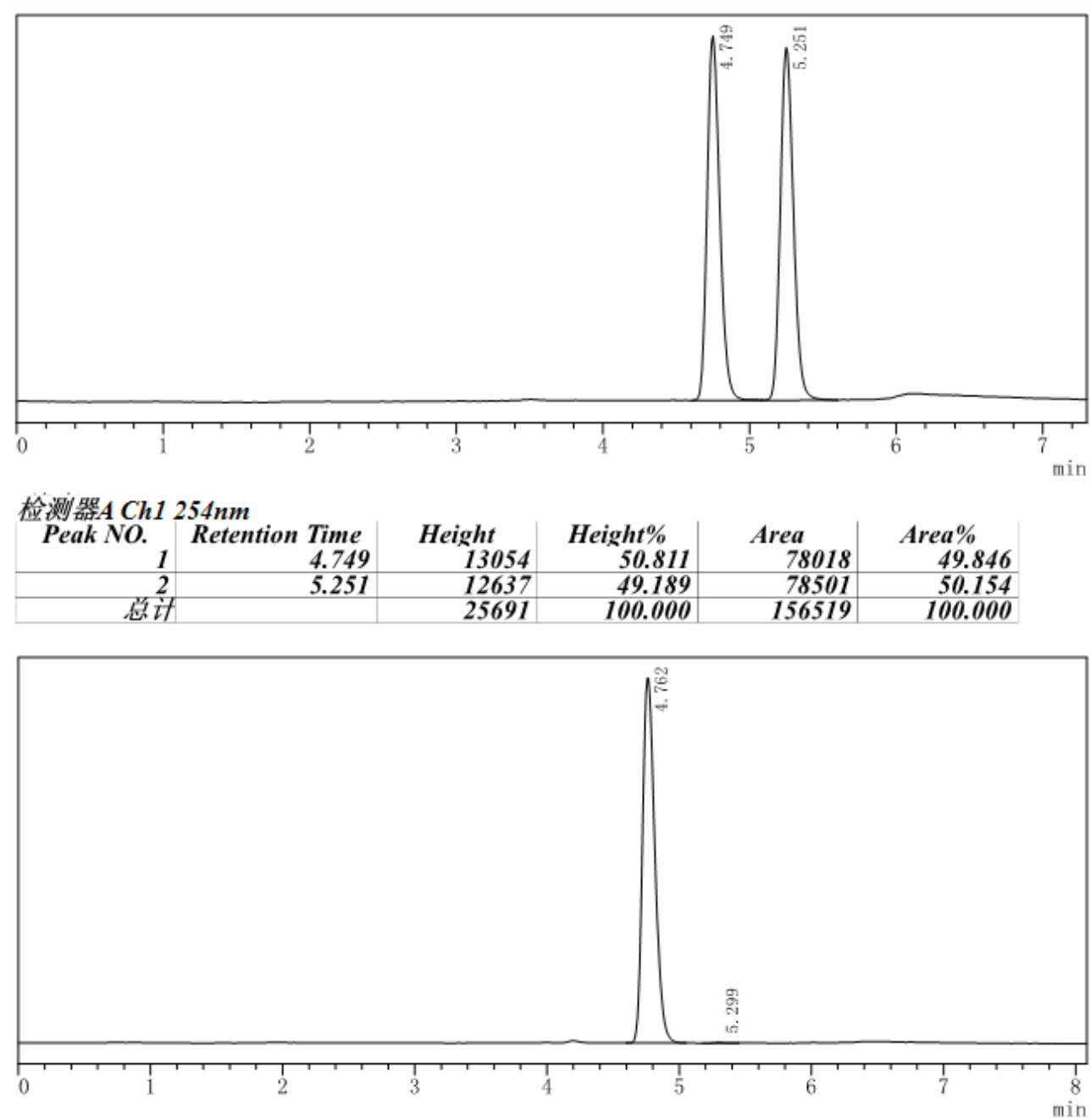

\begin{tabular}{|c|c|c|c|c|c|}
\hline Peak NO. & Retention Time & $\begin{array}{l}\text { Height } \\
27792\end{array}$ & $\begin{array}{l}\text { Height } \% \\
\quad 99.798\end{array}$ & $\begin{array}{l}\text { Area } \\
179689\end{array}$ & $\begin{array}{l}\text { Area } \% \\
\quad 99.811\end{array}$ \\
\hline 2 & 5.299 & 56 & 0.202 & 341 & 0.189 \\
\hline 总计 & & 27848 & 100.000 & 180030 & 100.000 \\
\hline
\end{tabular}




\section{References:}

(1) Jiang, Y.; Jiang, Q.; Zhu, G.; Zhang, X. Tetrahedron Lett. 1997, 38, 215.

(2) Yamagishi, T.; Ohnuki, M.; Kiyooka, T.; Masui, D.; Sato, K.; Yamaguchi, M. Tetrahedron: Asymmetry 2003, 14,3275 .

(3) (a) Li, C.; Breit, B. Chem. Eur. J. 2016, 22, 14655; (b) Zhou, Y.; Breit, B. Chem. Eur. J. 2017, 23, 18156; (c) Liang, L.; Xie, M.-S.; Qin, T.; Zhu, M.; Qu, G.-R.; Guo, H.-M. Org. Lett. 2017, 19, 5212; (d) Evans, P. A.; Uraguchi, D. J. Am. Chem. Soc. 2003, 125, 7158.

(4) Shu, C.; Leitner, A. Hartwig, J. F. Angew. Chem. Int. Ed. 2004, 43, 4797.

(5) Overman, L. E.; Owen, C. E.; Pavan, M. M.; Richards, C. J. Org. Lett. 2003, 5, 1809.

(6) Weiss, M. E.; Fischer, D. F.; Xin, Z.-Q.; Jautze, S.; Schweizer, W. B.; Peters, R. Angew. Chem. Int. Ed. 2006, 45,5694 .

(7) Cooke, M. L.; Xu, K.; Breit, B. Angew. Chem. Int. Ed. 2012,51, 10876.

(8) Gnamm, C.; Franck, G.; Miller, N.; Stork, T.; Brödner, K.; Helmchen, G. Synthesis 2008, 20, 3331.

(9) Meza, A. T.; Wurm, T.; Smith, L.; Kim, S. W.; Zbieg, J. R.; Stivala, C. E.; Krische, M. J. J. Am. Chem. Soc.

2018, 140, 1275. 